University of Louisville

ThinkIR: The University of Louisville's Institutional Repository

Electronic Theses and Dissertations

$8-2020$

\title{
Dynamic analysis and fabrication of a bi-stable structure designed for MEMS energy harvesting applications.
}

Masoud Derakhshani

University of Louisville

Follow this and additional works at: https://ir.library.louisville.edu/etd

Part of the Acoustics, Dynamics, and Controls Commons, Applied Mechanics Commons, ElectroMechanical Systems Commons, Energy Systems Commons, and the Manufacturing Commons

\section{Recommended Citation}

Derakhshani, Masoud, "Dynamic analysis and fabrication of a bi-stable structure designed for MEMS energy harvesting applications." (2020). Electronic Theses and Dissertations. Paper 3578.

https://doi.org/10.18297/etd/3578

This Doctoral Dissertation is brought to you for free and open access by ThinkIR: The University of Louisville's Institutional Repository. It has been accepted for inclusion in Electronic Theses and Dissertations by an authorized administrator of ThinkIR: The University of Louisville's Institutional Repository. This title appears here courtesy of the author, who has retained all other copyrights. For more information, please contact thinkir@louisville.edu. 
DYNAMIC ANALYSIS AND FABRICATION OF A BI-STABLE STRUCTURE DESIGNED FOR MEMS ENERGY HARVESTING APPLICATIONS

\author{
By \\ Masoud Derakhshani \\ B.Sc., Mechanical Engineering, University of Mazandaran, 2009 \\ M.Sc., Mechanical Engineering, Iran University of Science and Technology, 2011
}

A Dissertation Submitted to the Faculty of the

J. B. Speed School of Engineering of the University of Louisville In Partial Fulfillment of the Requirements for the Degree of

\author{
Doctor of Philosophy \\ in Mechanical Engineering \\ Department of Mechanical Engineering \\ University of Louisville \\ Louisville, Kentucky
}

August 2020 
Copyright 2020 by Masoud Derakhshani

All rights reserved 



\title{
DYNAMIC ANALYSIS AND FABRICATION OF A BI-STABLE STRUCTURE DESIGNED FOR MEMS ENERGY HARVESTING APPLICATIONS
}

\author{
By
}

Masoud Derakhshani

\begin{abstract}
A Dissertation Approved on
\end{abstract}
May 21, 2020

by the following Dissertation Committee:

Dissertation Director: Dr. Thomas A. Berfield

Dr. Kevin D. Murphy

Dr. Yanyu Chen

Dr. Shamus McNamara 


\section{ACKNOWLEDGMENTS}

I would like to express my sincere gratitude and appreciation to my doctoral advisor Dr. Thomas Berfield, who has been a true supervisor and a great mentor to me in the past several years and helped me a lot in this journey. I would like to thank him for all his support, guidance, and encouragement during my Ph.D. program.

I would also like to thank Dr. Kevin Murphy for all his support and supervision, especially in the modeling part of my work. As my co-advisor and one of my doctoral committee members, he has always encouraged me not to give up and I am truly thankful for it. I also wish to thank my other doctoral committee members, Dr. Shamus McNamara and Dr. Yanyu Chen, for their kind support, advice, and consideration during these years.

I should thank all our group members, Dr. Niknam Momenzadeh, Trung Hoang, Brian Allgeier, for all their help and support with my research. I would also like to thank the University of Louisville Micro/Nano Technology Center staff, especially Dr. Julia Aebersold and Curtis McKenna, as well as Conn Center for Renewable Energy Research staff, especially Dr. William Paxton and Dr. Jacek Jasinski, for their help and their collaboration. 
I would like to express my special thanks to my family, my mother, my father (may his soul rest in peace), and my three sisters, for always believing in me and being supportive in my whole life. This dream would have never become true without their help and support.

In the end, I would like to thank all my friends who have always been there for me and treated me like a family and a true friend whenever I needed their support. 


\title{
ABSTRACT \\ DYNAMIC ANALYSIS AND FABRICATION OF A BI-STABLE STRUCTURE DESIGNED FOR MEMS ENERGY HARVESTING APPLICATIONS
}

\author{
Masoud Derakhshani
}

May 21, 2020

Thanks to the rapid growth in demand for power in remote locations, scientists' attention has been drawn to vibration energy harvesting as an alternative to batteries. Over the past ten years, the energy harvesting community has focused on bistable structures as a means of broadening the working frequency range and, by extension, the effective efficiency of vibration-based power scavenging systems. In the current study, a new method is implemented to statically and dynamically analyze a bistable buckled, multi-component coupled structure designed specifically for low-frequency vibration energy harvesting systems in both macro and MEMS-scale sizes. Furthermore, several micro-fabrication steps using advanced manufacturing technology methods were applied to design and fabricate a micro-scale version of the energy harvester at the University of Louisville Micro/Nano Technology Center. First, previously efforts performed on different aspects of vibration energy harvesting systems are reviewed to show the current challenges associated with such devices. The coupled structure proposed in this project is then introduced and its equations of motion are developed based on nonlinear Euler-Bernoulli beam theory. These governing equations are discretized and solved using a Galerkin method in two different 
approaches: with some known shape functions which only satisfies the geometrical boundary conditions; with the exact shape functions obtained from solving the linearized coupled structure as a one single system. An experimental setup is also used to verify the advantages of designed structure in capturing bistable motion at low-frequency range. To validate the modeling approaches, the obtained results are compared with the ones captured from both FEA model and the experimental setup, which shows the superiority of the proposed approach in which exact shape functions of the system are used as the basis in the discretization process. After the validation of the proposed approach, it is applied on a micro-scale version of the system in which structural, piezoelectric, and electrode layers are all considered as they exist in an actual device. Furthermore, a different bistable system, which was previously studied by other researchers in the area, is analyzed by this method to show the reliability of the proposed model. For all these cases, the amplitude-frequency response of the system and snap-through regime with the variation of various parameters, including exciting frequency, base vibration, and buckling loads are investigated based on the developed model. It is shown that bisatble motion and other nonlinear phenomena such as super-harmonic behavior in the system can be captured under certain circumstances, which can significantly impact major system functionalities such as output voltage response and is crucial for the performance of energy harvesting devices. As mentioned above, various micro-fabrication techniques were also used to design and fabricate a microscale version of the proposed system, which eventually led to the successful fabrication of a MEMS device as a result of experimental efforts performed to overcome the challenges and issues associated with the designed manufacturing process. 


\section{TABLE OF CONTENTS}

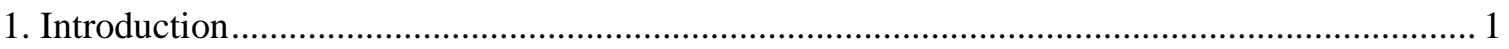

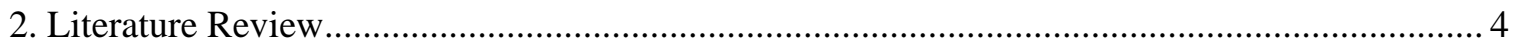

2.1. Background and Motivations ..................................................................................... 4

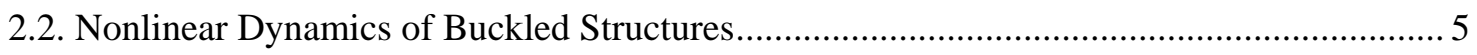

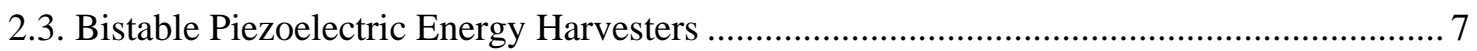

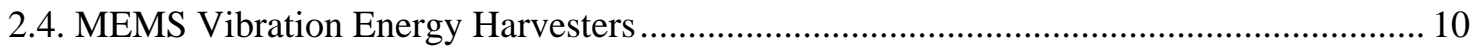

2.5. Proposed Structure for Bistable Vibration Energy Harvesting ........................................ 14

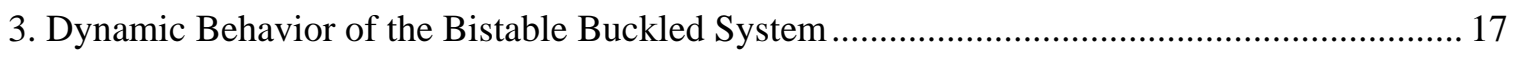

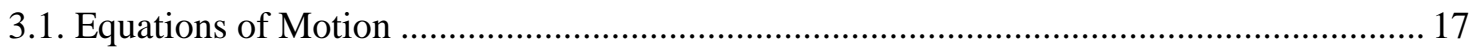

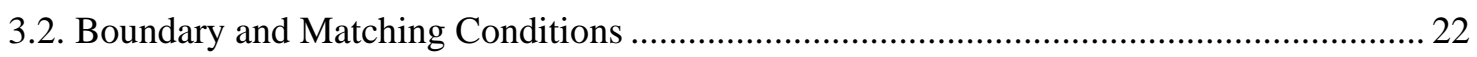

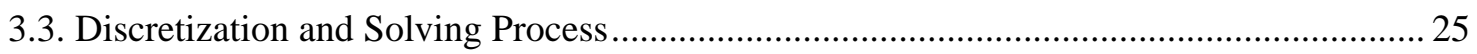

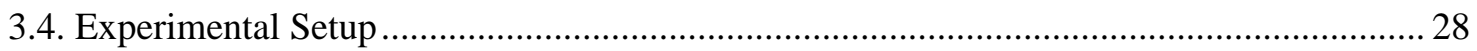

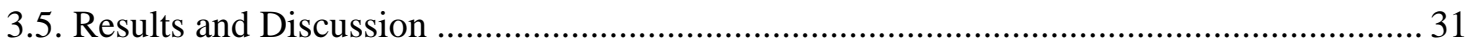

4. Component Coupling Method to Model the Bistable Structure.............................................. 35

4.1. Approximate Coupling Methods............................................................................. 35

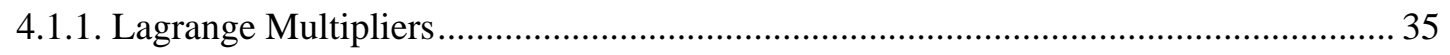

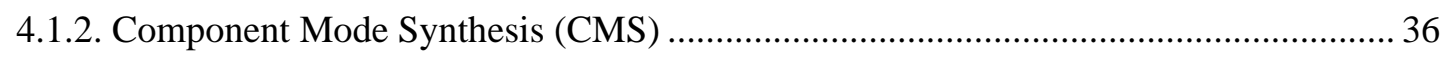

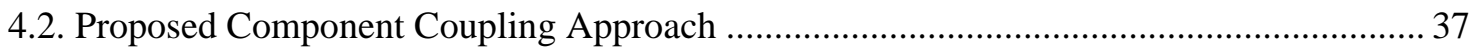


4.3. Formulation and Solving Procedure

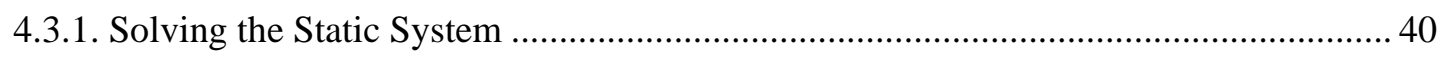

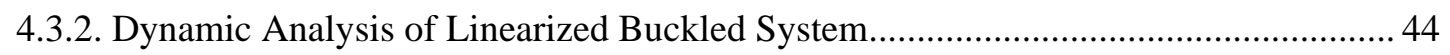

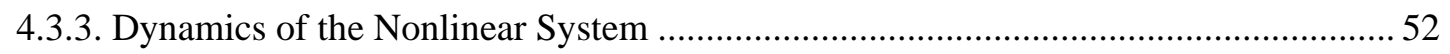

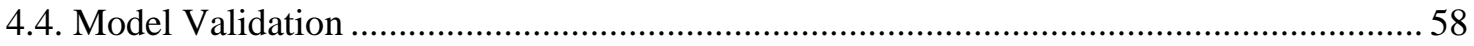

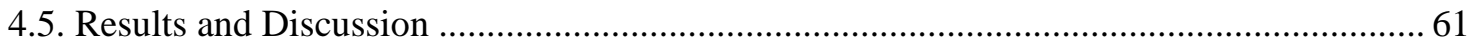

5. Mechanical Elongation and Output Power Prediction of a MEMS System .............................. 74

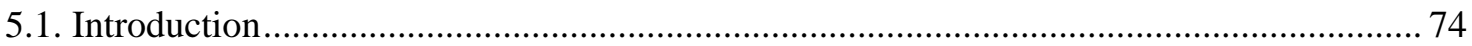

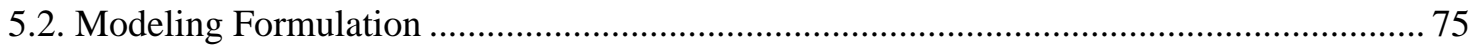

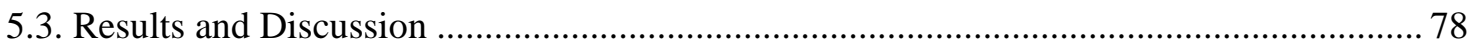

6. Analysis of a PVDF-Based Clamped-Clamped Buckled Structure …......................................... 89

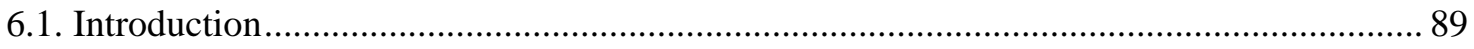

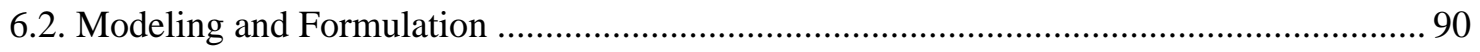

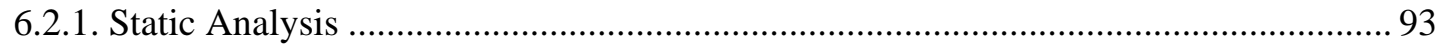

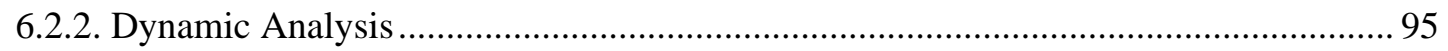

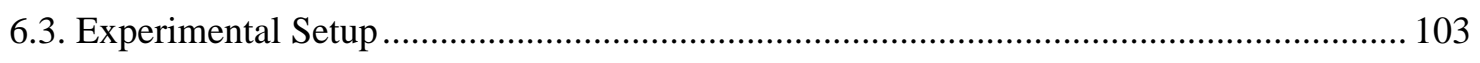

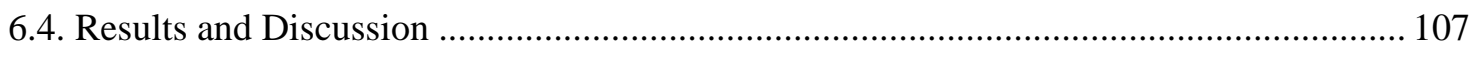

7. Microfabrication of the Designed MEMS Bistable Energy Harvester .................................... 117

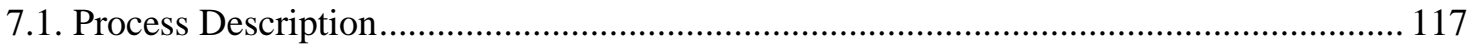

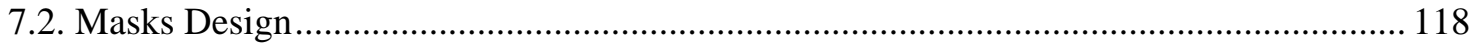

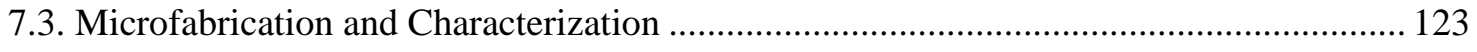


7.3.1. Photolithography

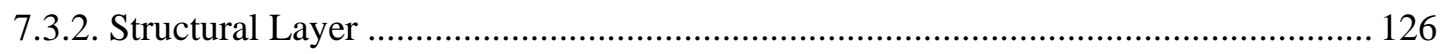

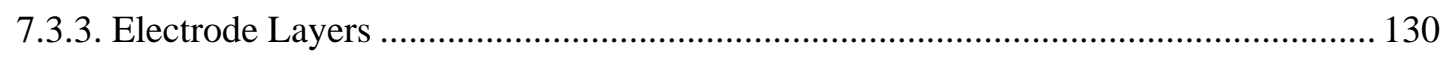

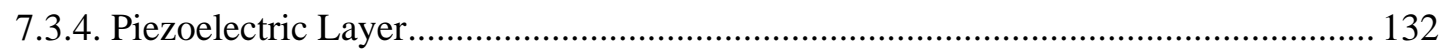

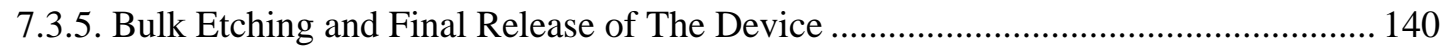

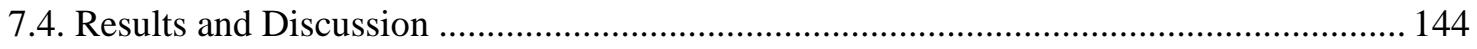

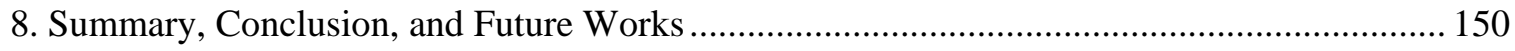

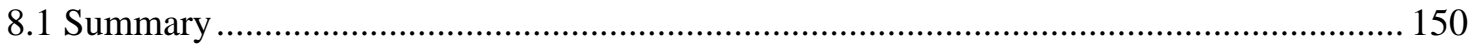

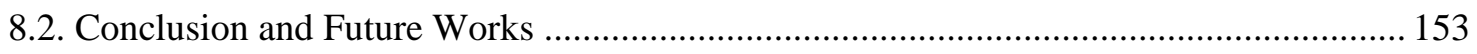

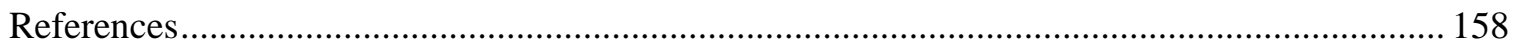

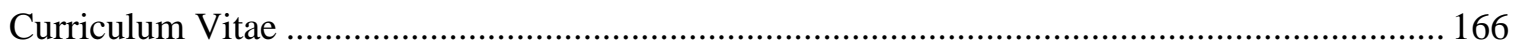




\section{LIST OF TABLES}

Table 1. Dimensions and physical parameters of the experimental setup.

Table 2. Critical buckling load and natural frequencies of the experimentally tested device (frequencies of higher modes are beyond the limit of the shaker table). 59

Table 3. First five natural frequencies obtained for the MEMS-scale bistable energy harvester. . 81

Table 4. Experimental sample dimensions and mechanical/electrical properties. 106

Table 5. Critical buckling load and first three natural frequencies obtained for the experimental sample. 109 


\section{LIST OF FIGURES}

Figure 1. The schematic model of the structural system for bistable vibration energy harvesting.

Figure 2. Two-dimensional view and parametric dimensions of each component.

Figure 3. Perspective view of a general Euler-Bernoulli beam in its local coordinate system...... 20

Figure 4. The experimental setup: (a) custom-built shaker table with the optoNCDT 1401 measuring sensor. (b) testing device mounted on the shaker table.

Figure 5. Amplitude-frequency response of the midpoint of the central beam for both modeling and experimental results.

Figure 6. Change of vibration amplitude of the buckled main beam vs. buckling level for $25 \mathrm{~Hz}$ exciting frequency

Figure 7. A schematic view of a single beam with different material properties.

Figure 8 . The coupled structure divided into eight separate parts.

Figure 9. Schematic view of three connections in the system: a) main beam-torsional rod. b) torsional rod-first cantilever arm. c) torsional rod-second cantilever arm.

Figure 10. Three post buckling states (two buckled stable states and one zero unstable state) of the central beam for the experimental device under compressive load $P=40 \mathrm{~N}$.

Figure 11. Amplitude-frequency response of the central buckled beam of the experimental device with $35 \mathrm{~N}$ buckling load and $2 \mathrm{~mm}$ base excitation. 61 
Figure 12. Amplitude-frequency response of the central buckled beam of the experimental device with four different base excitations for the buckling load $P=35 \mathrm{~N}$.

Figure 13. Amplitude-frequency response of the central buckled beam of the experimental device with four different base excitations for the buckling load $P=45 \mathrm{~N}$.

Figure 14. Amplitude-frequency response of the central buckled beam of the experimental device with four different base excitations for the buckling load $P=55 \mathrm{~N}$.

Figure 15. Modeling results of base excitation versus driving frequency showing regimes of snapthrough (ST) and no snap-though (NST) behavior for the experimental device with the buckling $\operatorname{load} P=35 N$

Figure 16. Modeling results of base excitation versus driving frequency showing regimes of snapthrough (ST) and no snap-though (NST) behavior for the experimental device with the buckling $\operatorname{load} P=45 N$

Figure 17. Modeling results of base excitation versus driving frequency showing regimes of snapthrough (ST) and no snap-though (NST) behavior for the experimental device with the buckling $\operatorname{load} P=55 N$ 68

Figure 18. Snap-through regime plot of the experimental device for buckling load vs exciting frequency for harmonic base vibration with the amplitude of $5 \mathrm{~mm}$.

Figure 19. Snap-through regime plot of the experimental device for buckling load vs exciting frequency for harmonic base vibration with the amplitude of $10 \mathrm{~mm}$. 70

Figure 20. Snap-through regime plot of the experimental device for buckling load vs exciting frequency for harmonic base vibration with the amplitude of $15 \mathrm{~mm}$. .71

Figure 21. Snap-through regime plot of the experimental device for base vibration amplitude vs buckling load at $23.5 \mathrm{~Hz}$ exciting frequency. 72 
Figure 22. Schematic cross-sectional area and the distance of the piezoelectric layer from the neutral axis 76

Figure 23. The designed MEMS-scale bistable energy harvester: a) top-view b) side-view 80

Figure 24. Different layers of the main buckled beam and its equivalent cross-sectional area parameters... 80

Figure 25. Variation of required base amplitude for the snap-through motion versus exciting frequency.

Figure 26. Variation of the main beam elongation amplitude versus central beam compressive stress level (normalized by the critical buckling stress) subjected to vibration sources amplitudes corresponding to the minimum required for bistable motion at each stress level, applied at $115 \mathrm{~Hz}$ excitation frequency. 84

Figure 27. Variation of main beam elongation versus base excitation for three different initial buckling stress levels at $115 \mathrm{~Hz}$ excitation frequency. .85

Figure 28. Snap-through regime plot of the MEMS-device for buckling stress versus exciting frequency. 87

Figure 29. Side view of the PVDF-based, buckled bistable system for vibration energy harvesting.

Figure 30. Schematic view of the whole system divided into its subcomponents. 93

Figure 31. Equivalent circuit design for the bistable vibration energy harvester with an external resistive load. 102

Figure 32. a) Buckled beam sample made of commercial PVDF with a central mass, b) the whole experimental setup of the shaker table and the laser sensor. 104 
Figure 33. Schematic view of static bending test on a cantilever sample of the commercial PVDF sheet: a) side view, b) cross-sectional view. 105

Figure 34. Schematic view of the free vibration test on a cantilever sample of the commercial PVDF sheet. 106

Figure 35. Output voltage response of the free vibration test on the commercial PVDF cantilever sample for $R=0.8 M \Omega$.

Figure 36. Schematic view of the static deflection and its corresponding displacement of the movable ending fixture. 108

Figure 37. Vibrational amplitude response of the central mass for base amplitude of $0.6 \mathrm{~mm}$ and frequency of $18 \mathrm{~Hz}$ : (a) experimental result; (b) modeling result; (c) Poincare plot.

Figure 38. Voltage response of the bistable sample for base amplitude of $0.6 \mathrm{~mm}$ and frequency of $18 \mathrm{~Hz}$ : (a) experimental result; (b) modeling result.

Figure 39. Vibrational amplitude response of the central mass for base amplitude of $0.75 \mathrm{~mm}$ and frequency of $24 \mathrm{~Hz}$ : (a) experimental result; (b) modeling result; (c) Poincare plot.

Figure 40. Voltage response of the bistable sample for base amplitude of $0.75 \mathrm{~mm}$ and frequency of $24 \mathrm{~Hz}$ : (a) experimental result; (b) modeling result.

Figure 41. Vibrational amplitude response of the central mass for base amplitude of $0.45 \mathrm{~mm}$ and frequency of $30 \mathrm{~Hz}$ : (a) experimental result; (b) modeling result; (c) Poincare plot. 114

Figure 42. Voltage response of the bistable sample for base amplitude of $0.45 \mathrm{~mm}$ and frequency of $30 \mathrm{~Hz}$ : (a) experimental result; (b) modeling result. 114

Figure 43. Amplitude-frequency response of the studied experimental bistable sample 115

Figure 44. Voltage-frequency response of the studied experimental bistable sample. 115 
Figure 45. Schematic view of the required steps for micro fabrication process of the MEMS-scale bistable energy harvester. 118

Figure 46. The photomask layout corresponding to the stressed silicon nitride as the structural layer of the device.

Figure 47. The photomask layout corresponding to the molybdenum as the bottom electrode layer of the device. 121

Figure 48. The photomask layout corresponding to the molybdenum as the top electrode layer of the device.

Figure 49. The photomask layout corresponding to the aluminum nitride as the piezoelectric layer of the device.

Figure 50. The photomask layout corresponding to the bulk etching of the top fabricated device in the DRIE process.

Figure 51. All five photomask layouts superimposed on the top of another. 123

Figure 52. The Headway Spinner and the hotplate located in the photolithography bay at the UofL Micro/Nano Technology Center. 125

Figure 53. The SUSS Mask Aligner located in the photolithography bay at the UofL Micro/Nano Technology Center. 126

Figure 54. Schematic view of the fabricated device for DRIE process: a) before final release; b) after final release. 127

Figure 55. Oxford PECVD PS100 machine located in the deposition bay at the UofL Micro/Nano Technology Center. 128

Figure 56. Toho FLX-2320-S thin film measurement machine located in the deposition bay at the UofL Micro/Nano Technology Center...... 129 
Figure 57. ICP/RIE Trion Metal Etcher located in the etching bay at the UofL Micro/Nano Technology Center.

Figure 58. Kurt J. Lesker PVD 75 Sputtering machine located in the deposition bay at the UofL Micro/Nano Technology Center.

Figure 59. XRD results for three samples of aluminum nitride thin film deposited on a molybdenum substrate with various sputtering parameters. 136

Figure 60. XRD results for the MOCVD deposited gallium nitride on a molybdenum substrate. 138

Figure 61. Deep reactive ion etching (DRIE) machine located in the etching bay at the UofL Micro/Nano Technology Center.

Figure 62. Microscopic topside view of the fabricated MEMS bistable energy harvester before doing the backside bulk etching process. 145

Figure 63. Backside view of the fabricated wafer after the DRIE process. 146

Figure 64. Backside microscopic images of some of the fabricated devices after the DRIE process.

Figure 65. A topside microscopic image of the final fabricated device after the final release.... 148

Figure 66. A schematic view of an extended version of the studied bistable energy harvester with a longer central beam and four cantilever arms. 154

Figure 67. Microscopic pictures of the designed extended version of the bistable energy harvester after the final release. 155

Figure 68. Microscopic pictures of some broken devices after the final release. 156 


\section{INTRODUCTION}

Many environments feature natural vibration sources that, if properly harnessed, offer the potential to serve as an energy source for a variety of light-duty electronic devices. This project aims to investigate the dynamic response of a unique-designed bistable structure proposed to work as a vibration energy harvester suitable for low-frequency range. In chapter two, a thorough literature review of previous works on macro/micro scale bistable vibration energy harvesters is presented with an emphasis on methods developed for dynamic modeling of nonlinear structures and the efforts to solve the challenges associated with the current energy harvesting systems. In the last section of this chapter, the proposed structure designed for bistable energy harvester is introduced and its approach to resolving issues encountered at low-frequencies by typical vibration energy harvesters is described in detail.

In chapter three, the dynamic response of the proposed bistable structure under harmonic excitation is analyzed. First, the equations of motion are derived based on nonlinear EulerBernoulli beam theory, and the modeling simplifications considered for each part of the system are shown. Then, a Galerkin method is applied to discretize the obtained nonlinear equations with the functions which only satisfy the essential geometrical boundary conditions of the system. A Runge-Kutta numerical algorithm is used to directly solve the discretized nonlinear ordinary differential equations. In order to verify the proposed model, 
testing results from a macro-scale experimental setup are presented in the second half of this chapter. While the experimental results show the benefits of the proposed design as a low-frequency driven bistable harvester under certain conditions, some inconsistency is observed between the developed model and experiment.

Resolving this inconsistency requires a component coupling method that is able to satisfy all of the boundary conditions at the connections responsible for coupling different components in the system together. To do so, a new approach is developed in chapter 4 to accurately solve both the nonlinear static and dynamic equation of the coupled structure. After model verification with both finite element analysis and experimental results, the amplitude-frequency responses and snap-through regimes plots of the tested bistable device were developed based on the proposed approach. Results show the effects of different parameters of the system, including base excitation features and compressive buckling stress level for the central beam, on the bistable energy harvester performance.

In the chapter five, the proposed method is presented as applied to an actual MEMS system made of structural, piezoelectric, and electrode layers which allows investigation of the mechanical elongation and power output prediction of the micro-scale energy harvesting system. Using dimensions and material layers of the designed MEMS device, a parametric analysis was performed to investigate the strain development within the piezoelectric layer as related to snap-through behavior of the buckled beam under different dynamic loading conditions. The results showed that the initial beam buckling stress and the base excitation amplitude are two major influences on the predicted vibration energy harvester performance. 
To show the robustness of the developed coupling component method, chapter six studies the implementation of this technique on a classic bistable system, a buckled beam with a central mass attached to its middle. The major difference between the considered system and previously studied versions is that the bulk part of the energy harvester is made of polyvinylidene fluoride (PVDF), which is a piezoelectric polymer that necessitates the consideration of coupled mechanical-electrical governing equations in the modeling approach. By comparing the modeling results for the described system with the those captured from experimental tests, it is shown that the presented method can successfully predict the nonlinear response and output voltage of the system as well as some nonlinear characteristics such as super-harmonic behavior in both monostable and bistable regimes.

In chapter 7 , the microfabrication procedure for producing the structural design at the MEMS scale is detailed. First, the designed masks and the overall fabrication process is schematically described in this chapter. Then, several steps required for the deposition and patterning of each layer as well as the characteristic tests needed to clarify the performance of the deposited layers are fully investigated. The challenges associated with each step are explained in detail, particularly for the most critical facet of the device, the piezoelectric aluminum nitride layer. After numerous iterations of the experimental efforts, a successful fabrication process to produce the micro-scale system was established.

In the final chapter, conclusions and a summary of the major contributions of this project are presented. Finally, suggested future works and extensions of the current study are discussed to close the dissertation. 


\section{LITERATURE REVIEW}

\subsection{Background and Motivations}

Environmental concerns associated with conventional battery technologies, coupled with growing demand for small electronic sensors and devices, make energy harvesting systems an intriguing power supply option in many applications. For cases in which solar energy is not a viable alternative, vibration-based energy harvesting devices offer a comparable energy density solution provided the application environment features a vibration source from which power can be scavenged. Moreover, vibration-based energy harvesting offers tremendous value for powering Wi-Fi connected sensors, especially when located in difficult-to-access locations.

The first studies of vibration energy harvesting have begun by trying to tune the first natural frequency of the device with the ambient vibrations to get the maximum output power from such systems. However, it has been turned out that these ambient vibration sources are typically very different from laboratory-controlled driving vibration conditions, which makes linear vibration energy harvesters not very efficient for practical situations. Most real-life sources ripe for harvesting involve vibrations of structures at low acceleration magnitudes acting randomly over a wide bandwidth of low frequencies. To better accommodate the vibration sources associated with these realistic working conditions, researchers have replaced simplistic, linear energy harvesting devices with nonlinear ones. 
Structures with nonlinear dynamic responses such as bistable beams, domes, etc., have been shown to broaden the working frequency range of vibration energy harvesters to function in these chaotic driving vibration environments [1-8].

Numerous studies have concentrated on bistable oscillators as a promising way to address the issues associated with the linear Vibration Energy Harvesters (VEH), including covering a wider bandwidth of frequency and the existence of two stable states that promote a more reliable design for energy harvesting systems. Harne and Wang [3] gave a thorough review on the principal concepts of dynamically nonlinear beams as bistable energy harvesters. They also pointed out the challenges with different analysis approaches and popular experimental setups in their study.

\subsection{Nonlinear Dynamics of Buckled Structures}

Using nonlinear phenomena in vibration energy harvesting systems as a method for improving limited frequency bandwidth has been considered in several previous studies [8-16]. To predict the power efficiency of bistable energy harvesting devices, there is a need for accurate analytical modeling of the nonlinear behavior of their internal structures. While the dynamic analysis of such structures is not new, many difficulties with the existing modeling approaches emerge as the complexity of the bistable energy harvesting devices increases. A clamped-clamped beam subjected to an axial buckling load is one such nonlinear, bistable component. The dynamic behavior of this system has been the subject of previous studies using methods that do not easily translate to more complex buckled structures [17-19]. Theoretical analysis of nonlinear dynamics of a clampedclamped buckled beam using Galerkin discretization method was studied by Emam and Nayfeh [19]. It was shown that by considering only the first mode of vibration, the obtained 
results would not satisfy the experimental ones. However, by considering a greater number of modes for the nonlinear beam, its dynamic behavior can be better described for each sequence of motion. This group has also investigated the nonlinear dynamic response of a clamped-clamped beam with respect to the primary and subharmonic resonances of the first vibration mode [17]. In this work, they used Galerkin's method to discretize the governing partial differential equation at multiple scales and a shooting method was implemented to get the approximate local and global solutions, respectively. Different dynamic behaviors were observed via analytical modeling, which was compared with some experimental results. The solution of the post-buckled configuration of a nonlinear beam with three different end boundaries: fixed-fixed, fixed-hinged, and hinged-hinged beams was investigated by them in [20]. A closed-form expression was formed for the static postbuckled beam, in which the critical buckling load and static mode shapes were obtained as a result of the static response. They found out that the first buckling mode was a stable one for all three boundary condition types. Dynamic modeling and active control of a highly nonlinear hinged-hinged beam was studied by Nbendjo and Woafo [21]. In this study, it was shown that when the beam is not highly pre-loaded the classical Duffing oscillator may be a good choice to simulate the nonlinear behavior, while adding another term of nonlinearity to the potential function can explain the system more exactly. However, the major difference of this model with the classical Duffing oscillator is its ability to describe the dynamical destruction in the system for large deformations in the potential function.

Another study on the nonlinear dynamic modeling of buckled beam was performed by Vangbo [22], among others [23-26]. In this study, the static displacement equation of the beam was found using classical Euler-Bernoulli theory and the dynamic deflection of the 
beam about the buckled configuration was found as a function of applied external force using the shape functions of the fixed-fixed beam coupled with the Lagrangian.

Linear dynamic behavior of coupled component structures has been previously analyzed in several works. In these studies, the linear vibration of each component was individually investigated and the coupling process was satisfied via applying boundary conditions [2729]. Other related previous works on nonlinear behavior of beams include a study by Ghayesh, et al. [18] on the post-buckling behavior of an axially moving beam, an investigation of the nonlinear dynamics of a fixed-fixed beam energy harvester with one longitudinal moving end attached to a tip mass by Garg and Dwivedy [30], and work on a nonlinear bistable oscillator based on a double buckled beam with a tip mass and elastic boundary conditions by Liu et al. [31].

\subsection{Bistable Piezoelectric Energy Harvesters}

Piezoelectric-based energy harvesters remain the dominant type of VEH system due to continued improvements in manufacturing processes and material efficiencies. A classic approach to achieve better power performance in real-life application environments that often feature non-stable driving frequencies is to broaden the viable energy harvesting frequency domain. One such notable broadband piezoelectric energy harvester studied by Erturk and Inman [32] was created using a bistable duffing oscillator in which a magnetic field was utilized to broaden the non-resonant operating frequency. In this study, the authors showed that a piezomagnetoelastic configuration can generate more output power with a wider bandwidth compared to similar, commonly employed piezoelectric harvesters. Stanton et al. [33] studied the effects of nonlinearity in vibration energy harvesting on the broadband frequency response both theoretically and experimentally. The system studied 
in this paper was a typical cantilever with bimorph lead zirconate titanate (PZT) layer and a concentrated mass with a magnetic field to induce bistability into the device. They found out that bistable behavior can result in getting a broader band frequency response compared to linear energy harvesters.

The dynamic analysis of piezoelectric buckled clamped-clamped beam for vibration energy harvesting was investigated by Cottone 1 et al. [34]. In this study, the bistability was induced by axial compressive force. Both numerical and experimental data showed that bistable buckled beam has a more reliable performance in vibration energy harvesting compared to the linear one. Vocca et al [35]. compared cantilever vs clamped-clamped beam for their nonlinear dynamic behavior. The results showed that the nonlinear beam has better output voltage than the linear one. It was also shown that nonlinear cantilever generally has higher output power compared to buckled beam for exponential noise input. Different parameters of bistable piezoelectric buckled beams were investigated for two different boundary conditions, clamped-clamped and simply supported beam in [36]. It was shown that for both boundary conditions, the buckled state has a better output compared to the linear one and the clamped beam has higher output voltage while it is on its bistable mode. Friswell et al. [37] studied a new configuration of vibration energy harvester, in which a cantilever beam with a tip mass is mounted vertically and excited in the transverse direction at its base. The results showed that by increasing the tip mass, the nonlinear behavior mostly dominates the system until it ends up switching to bistable configuration. Dynamic response of a laminated piezoelectric beam in both monostable and bistable conditions was investigated for energy harvesting by $\mathrm{Li}$ and Qin [38]. It was proven that for both harmonic excitation and Gaussian random noise bistable buckled beam has a better 
performance compared to the unbuckled one. Blarigan et al. [39] developed a model for describing the dynamic responses of a non-uniform bistable piezoelectric beam used for vibration energy harvesting. The necessity of keeping several modes in addition to the buckled equilibrium mode shape in the analysis in order to properly capture the small strain snap-through effects of the system was found in this study.

Effects of piezo-magnetoelastic harvesters were investigated and compared in different cases for achieving the best performance of the energy harvesters at low frequencies by Abdelkefi and Barsallo [40]. It was shown that the attractive interaction and smaller spacing gap between two magnets is very beneficial to design a low-frequency piezoelectric energy harvester due to its softening behavior. As shown in this part, there have been a lot of studies on the dynamic behavior of bistable beams as a piezoelectric based energy harvesting system; however, in most of the works, a single nonlinear beam was considered in the modeling part. The main drawback of using such structure as an energy harvester is the difficulty of changing states between two stable conditions during dynamic motions.

Another critical component to translating the findings from these efforts into production is tuning the individual energy harvesters to specific application environments. For nonlinear structures, this often means introducing certain innovative design elements or selecting piezoelectric smart materials with high-strain tolerances to accommodate large structure deflections in order to reach optimal natural frequencies for many common harvesting environments (generally in the range below $100 \mathrm{~Hz}$ ) [41, 42]. While prior works have analyzed power output as a means to improve harvester dimensional design parameters [43-45], these often focus on electrode arrangement or external circuit designs and rarely 
consider nonlinear structure deformations or material processing. For example, the processing of the piezoelectric polymer polyvinylidene fluoride (PVDF) used in this study (chapter six) can demonstrate a wide range of electromechanical performance, dependent on the relative content of the different semi-crystalline phases present.

Fabrication of 2D PVDF structures such as films or sheets is usually accomplished by dissolving pellets or powder in a solvent and spin casting as a film, or by melting/hotpressing a bulk amount of raw material. Of the five different polymorphs microstructures exhibited by PVDF, the $\beta$-phase is noted for demonstrating the most substantial electromechanical coupling [46]. Different approaches have been investigated to enhance the piezoelectric behavior by inducing greater $\beta$-phase content, in particular using mechanical stretching of the material to convert $\alpha$-phase (the dominant phase in PVDF upon standard processing) into $\beta$-phase [47-49]. This mechanical stretching route is used for the major of the commercially produced piezoelectric PVDF sheets. During the application of stresses to the PVDF material, the environmental temperature [50], the quench rate [51], and the water/solvents selected [52] are all key factors that can influence the relative content of the respective phases in the resulting sheets. Beyond its significant electromechanical performance, PVDF has been utilized in different a variety of applications and environments [53-57], with some special considerations for its biocompatibility [52, 58], its excellent resistance to acids [59, 60] and UV [61], as well as its bending, stretching and forming capabilities [53, 62].

\subsection{MEMS Vibration Energy Harvesters}


As stated in the first part of this section, one of the basic challenges for vibration energy harvesters is maximizing the power output of the system under what are typically chaotic, low-frequency vibration sources. The lower range of operating frequencies (less than $200 \mathrm{~Hz}$ ) is especially challenging for micro-scale energy harvesters due to natural frequency dimension scaling inefficiencies [1-3].

A number of works have concentrated on improving the operational frequency bandwidth and output power of MEMS-scale vibration energy harvesters, in particular through the use of nonlinear structures like buckled beams or plates. Betts et al. [63] studied both the static and dynamic response of uniquely-arranged bistable composite plates with bonded piezoelectric patches functioning as a broadband vibration energy harvester. They found that while the thicker laminate plates produce higher electrical energy when snap-through motion of the buckled plate occurs, the prevalence of such behavior is reduced due to the plate stiffness increase. The influence of bistable structure behavior in energy harvesting has been further explored in several studies by using an elastic support with an external magnet to help the system have the bistable motion at low-intensity vibrations [64, 65], optimizing the active piezoelectric area in the system [44], utilizing the softening nonlinearities of the piezoelectric materials to lower the operating frequency [66], and proposing a vertical configuration of cantilevered beam to work as an energy harvester [37].

Recent advances in the microfabrication processes have led to theoretical and experimental studies to enhance the efficiency of MEMS piezoelectric energy harvesters [67, 68]. Ando, et al. [13] have presented a nonlinear vibration energy harvester based on the snap-through 
motion being able to provide enough energy for an RF transmitter. The effects of nonlinearities on energy harvesting from a piezomagnetoelastic beam under random excitations was studied by De Paula, et al. [69]. Both numerical and experimental results showed the benefits of having snap-through motion in bistable structure systems compared to linear, monostable ones. The use of bistable thin walled cylindrical shells [70] and thin plates with multiple piezoelectric layers [71] have been also studied for optimized energy harvesting applications. Of direct significance to this work are additional studies evaluating strain stretching in a nonlinear clamped-clamped beam with a center proof mass [72] and assessing a buckled asymmetric piezoelectric beam as energy harvesting systems [14], both of which demonstrate the potential benefits of frequency bandwidth expansion.

Fang, et al. [73] designed and fabricated a MEMS-scale piezoelectric-based vibration energy harvester using different techniques of micro-fabrication. Their experimental testing of the fabricated devices showed that improved microfabrication methods could produce a good performance compared to typical micro-scale vibration energy harvesters. Liu, et al. [74] developed a MEMS-scale piezoelectric based power generator consisting of cantilevers array to improve the frequency range and power output. The structural material for the cantilever was silicon with PZT film as a transducer and a proof mass is attached to the free end of each beam. The experimental results showed that the arrayed design of cantilevers with different dimensions makes the system work at a broader range of frequencies and ultimately is able to generate more output power. Ferrari et al. [75] studied the efficiency enhancement of a nonlinear piezoelectric vibration energy harvester coupled with a permanent magnet. A piezoelectric bimorph cantilever was considered as the harvester. The tip mass of the cantilever was faced towards a fixed permanent magnet, 
which serves a role in creating bi-stability in the system. The experimental results showed that for a low enough distance between the tip mass and the magnet, a softening behavior could be captured which shifts the resonances towards lower frequencies. It was also observed that snap-through motion can be captured when the system is excited by whitenoise mechanical vibrations. In 2015, Rezaeisaray et al. [76] designed and fabricated a multi-degree of freedom system as a vibration energy harvester functional at low excitation frequencies. In their proposed design, a big proof mass was connected to the device frame by two cantilever beams. The structure was fabricated from a silicon substrate, and aluminum nitride was used as the electromechanical converter material. One of the advantages pointed out in this work was being able to reduce the first three natural frequencies of the micro-scale size of the system to within the range of ambient vibrations. The potential of a flexible structure consisting of multiple beams connected with joint masses in a zigzag combination as a vibration energy harvester was studied by Zhou et al. [77]. In this study, a theoretical model was proposed and compared with both finite element analysis and experimental results. The obtained results showed that the resonant frequencies of the system could be significantly decreased by increasing the number of the considered beams and, consequently could lead to a more practical device for lowfrequency ambient vibrations. Another advantage was pointed out in this research is the functionality of this device under multi-directional excitations, which makes it a suitable approach to harvest energy from human body motion.

As shown in above, while there have been numerous studies on the performance analysis and improvement of the micro-scale vibration energy harvesters, some unique challenges 
still exist for the modeling and optimization of small-scale bistable structure systems under dynamic loading conditions.

\subsection{Proposed Structure for Bistable Vibration Energy Harvesting}

As shown in this section, there have been a lot of works done on the design and modeling of the vibration energy harvesters to make them work in the real-life frequency range. However, most of the works that have been done so far include the typical bistable beam, in which snap-through motion is not achievable easily, especially for low exciting frequencies and low energy density. Considering this fact that having bistable motion at low-frequency range is the main concern with the current small-scale bistable energy harvesters, this work is trying to resolve this issue by modeling a unique structure, which is suitable to be used as a bistable harvester, while keeping the operational frequency of the device in the legitimate range for practical energy harvesters.

The bistable buckled vibration energy harvester described in this study (Figure 1) has a unique design among current vibration energy harvesting devices. Contrary to the typical bistable systems used as a vibration energy harvester, in which it is hard to capture the snap-through motion at low-frequency range, there are two cantilever arms with a lump mass at their ends designed in this coupled structure in a way that increases the whole system flexibility and helps it have bistable motion more easily. The large motion created in these arms is transferred to the main buckled beam via a torsional rod assisting the beam to switch between its stable states at low-frequencies. This type of design can be helpful especially for MEMS energy harvesters, in which operating frequency of the current devices is higher than real-life applications range. 


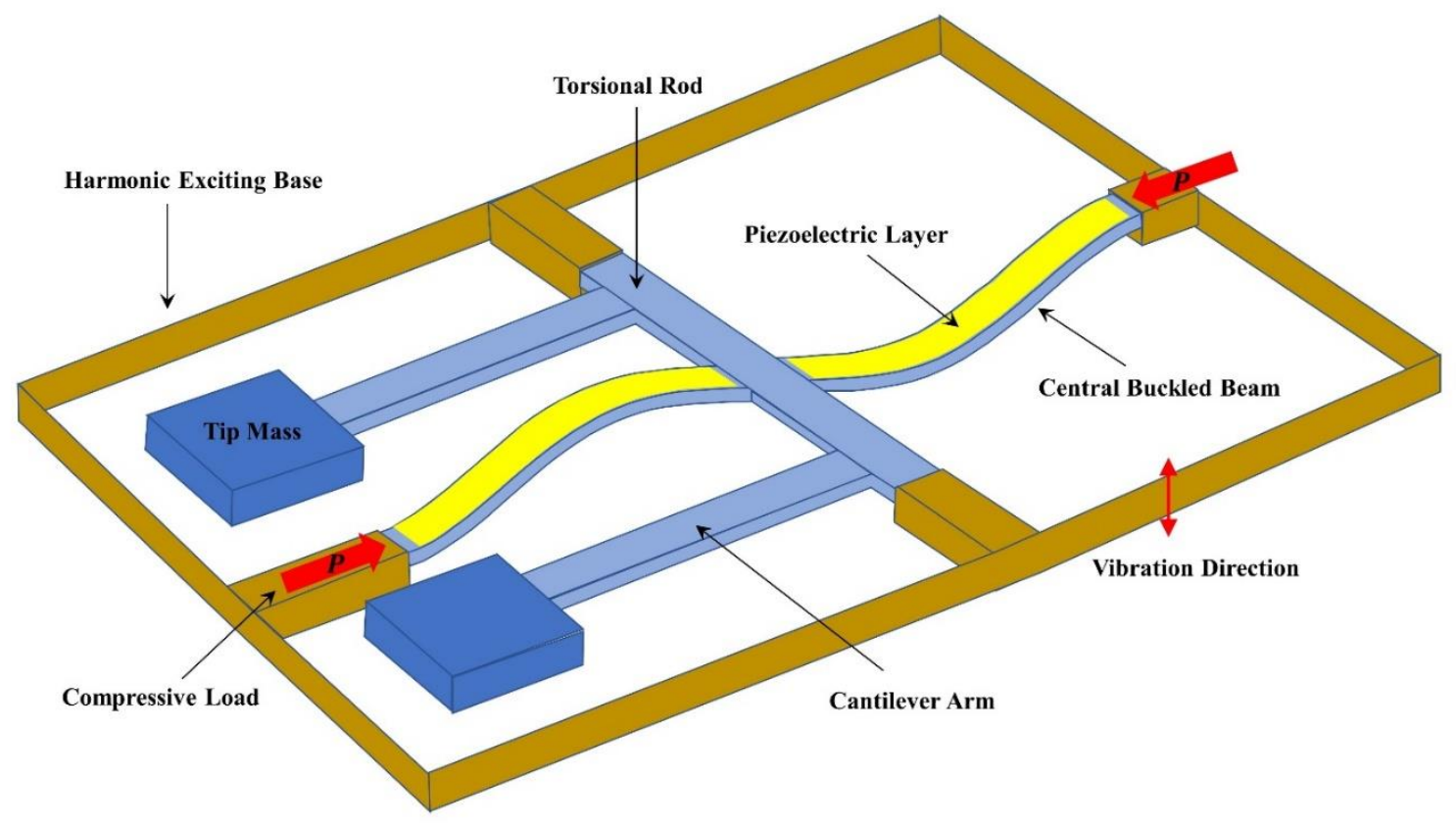

Figure 1. The schematic model of the structural system for bistable vibration energy harvesting.

A schematic model of the structure is shown in Figure 1, which includes a central buckled beam with clamped ends and a "quasi-pinned" condition at the midpoint. The buckling in the central beam is achieved by applying some compressive load, which makes the system bistable. The piezoelectric layer attached to the central beam has the role of converting mechanical strain into the electrical current and is shown in yellow in the figure. The central buckled beam is connected to two cantilever arms through a torsional rod, which provides the "pin constraint" for the centrally buckled beam. The rod acts as a torque arm for the system, transferring torque to the central beam via dynamic motion of the two identical cantilever arms with end mounted tip masses. The whole system is considered on a harmonically moving base, mimicking the driving environmental vibrations from which power is to be scavenged. 
In the next chapter. the designed structure and its functionality for helping induce snapthrough motion in low driving frequency domains is explained. The potential theories, assumptions and possible approaches for building a dynamic model that appropriately captures the behavior of this nonlinear system will be discussed. 


\section{DYNAMIC BEHAVIOR OF THE BISTABLE BUCKLED SYSTEM}

In this chapter, the dynamic analysis of the proposed coupled structure is considered. The equation of the motion for each part of the system is first derived using Hamilton's principle based on Euler-Bernoulli beam theory. There are some simplifications and assumptions considered for deriving the dynamic equations for each part. In the next section, Galerkin's method is implemented to discretize the obtained equations by applying some typical shape functions that satisfy the geometrical boundary conditions between the components of the system. Then, a macro-scale experimental setup is presented to test the functionality of the device and the obtained results are compared with the ones from the developed model. A comparison of results and a discussion of the discrepancies between the developed model and the experiment are included in the last section of this chapter.

\subsection{Equations of Motion}

As described in the previous chapter, the bistable buckled vibration energy harvester shown in Figure 1 has a unique design among all other current bistable energy harvesters. It consists of a clamped-clamped main buckled beam which is attached to a torsional rod in the middle. The rod plays as a torque arm for the system which transfers the dynamic motion of the two identical cantilevered arms with tip masses at their ends to the central buckled beam. The whole system is assembled on a harmonic moving base, which 
generates the required harmonic motion for the energy harvester. The parametric dimensions of the different components are shown in Figure 2.

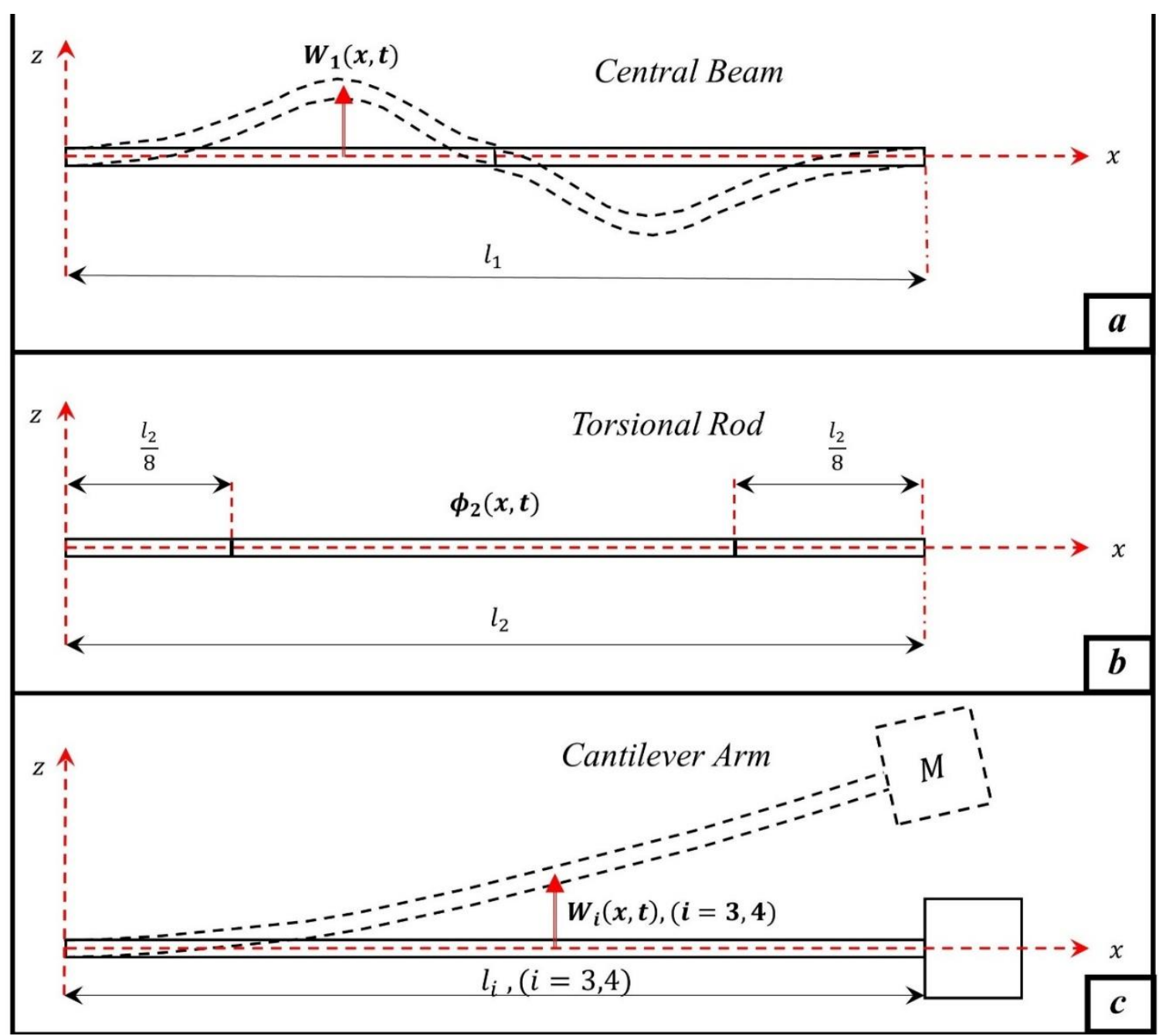

Figure 2. Two-dimensional view and parametric dimensions of each component.

The actual central beam is covered with piezoelectric layers on the top. Since the electromechanical coupling effects induced by the thin piezoelectric layers on the structural behavior of the central buckled beam are minimal (i.e., the piezoelectric layers have low piezoelectric constants and the stiffness of this layer is very small compared to that of the structural member material), the electrical part of the equation is neglected in our dynamic 
modeling. The bistability in the beam is provided by a central buckling load applied at the end of the central beam in the longitudinal direction. In addition, preliminary experimental testing showed that the nonlinear effects of the torsional rod and cantilever arms are negligible due to the considered geometry and range of driving frequencies. Considering the fact that bistability only occurs in the central beam makes this assumption valid for a moderately buckled situation. Thus, the only nonlinear behavior considered comes from the central buckled beam. Nonlinear Euler-Bernoulli beam theory with longitudinal and lateral deflection is considered for modeling of the beam. The strain relation for a classical nonlinear beam with a rectangular cross-sectional area (Figure 3) can be written as follows:

$$
\epsilon_{x x}=\left[\frac{\partial U}{\partial x}+\frac{1}{2}\left(\frac{\partial W}{\partial x}\right)^{2}\right]-y \frac{\partial^{2} W}{\partial x^{2}}
$$

where $U$ and $W$ are the longitudinal and vertical deformation of the beam respectively.

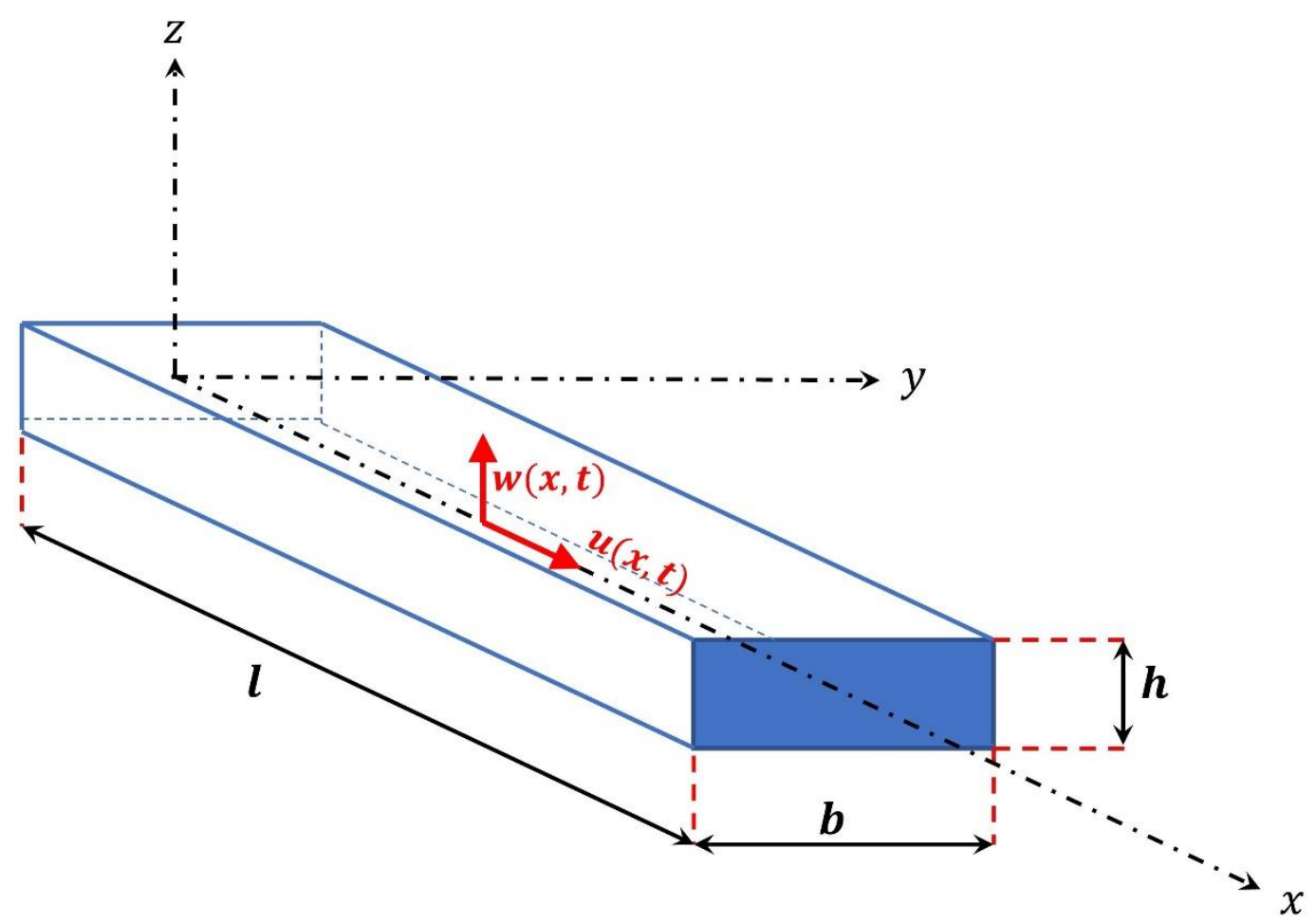


Figure 3. Perspective view of a general Euler-Bernoulli beam in its local coordinate system.

Hamilton's principle is used to derive the nonlinear dynamic equation of the buckled beam. To do so, the relations for kinetic and potential energy terms need to be formulated first:

$$
\begin{aligned}
& T E=\frac{1}{2} \int \rho \dot{\vec{V}} \cdot \dot{\vec{V}} d \mu \\
& U E=\frac{1}{2} \int E \epsilon_{x x}^{2} d \mu
\end{aligned}
$$

in which

$$
\vec{V}=U \underline{i}+W \underline{j}
$$

By substituting the nonlinear stress-strain relation (equation 1) into the energy terms, the following equations would be obtained:

$$
\begin{gathered}
T E=\frac{1}{2} \rho \int_{0}^{l_{1}}\left\{\dot{U}_{1}^{2} A_{1}+\dot{W}_{1}^{2} A_{1}+\left(\frac{\partial \dot{W}_{1}}{\partial x}\right)^{2} I_{y_{1}}\right\} d x \\
U E=\frac{1}{2} E \int_{0}^{l_{1}}\left\{A_{1}\left[\left(\frac{\partial U_{1}}{\partial x}\right)^{2}+\frac{1}{4}\left(\frac{\partial W_{1}}{\partial x}\right)^{4}+\left(\frac{\partial U_{1}}{\partial x}\right)\left(\frac{\partial W_{1}}{\partial x}\right)^{2}\right]+I_{y_{1}}\left(\frac{\partial^{2} W_{1}}{\partial x^{2}}\right)^{2}\right\} d x \\
W_{N C}=P . \Delta-W_{C_{1}}
\end{gathered}
$$

$T E, U E$, and $W_{N C}$ are the kinetic, potential and non-conservative energy of the central nonlinear beam respectively. In order to avoid any confusion in the future, all the displacements of the central beam are denoted by $1 . A_{1}, I_{y_{1}}, P, \Delta$, and $W_{C_{1}}$ are crosssectional area, second moment of area about $y$ axis, applied buckling load, total 
longitudinal displacement of the beam, and amount of work done by the damping force respectively. Applying Hamilton's principle to the obtained energy terms would give us the nonlinear Euler-Bernoulli equation for the central beam as follows:

$$
\begin{gathered}
\bar{m}_{1} \frac{\partial^{2} W_{1}}{\partial t^{2}}+E I_{y_{1}} \frac{\partial^{4} W_{1}}{\partial x^{4}}+P \frac{\partial^{2} W_{1}}{\partial x^{2}}+C_{1} \frac{\partial W_{1}}{\partial t}-\frac{E A_{1}}{2 L_{1}} \frac{\partial^{2} W_{1}}{\partial x^{2}}\left[\int_{0}^{l_{1}}\left(\frac{\partial W_{1}}{\partial x}\right)^{2} d x\right] \\
=-\bar{m}_{1} \ddot{W}_{B}
\end{gathered}
$$

where parameters $\bar{m}_{1}, C_{1}$, and $W_{B}$ are mass per length, damping coefficient, and base vibration respectively. Doing the same procedure for the torsional rod and cantilever arms, the equations of motion for the other components are obtained as bellow:

$$
\begin{gathered}
\rho J_{p} \frac{\partial^{2} \phi}{\partial t^{2}}+C_{2} \frac{\partial \phi}{\partial t}-G \gamma \frac{\partial^{2} \phi}{\partial x^{2}}=0 \\
\bar{m}_{i} \frac{\partial^{2} W_{i}}{\partial t^{2}}+E I_{y i} \frac{\partial^{4} W_{i}}{\partial x^{4}}+C_{i} \frac{\partial W_{i}}{\partial t}=-\bar{m}_{i} \ddot{W}_{B} ; \quad(i=3,4)
\end{gathered}
$$

in which $\phi, W_{3}$, and $W_{4}$ are the rotation of the torsional rod and vertical displacement of each cantilevered arm respectively. Parameters $J_{p}$, and $G$ are the polar second moment of area for a rectangular cross-section and shear module of elasticity for the torsional rod. Dividing the equations (8-10) by $\bar{m}$ gives us the following relations:

$$
\begin{gathered}
\ddot{W}_{1}+\bar{K}_{1} W_{1}^{\prime \prime \prime \prime}+\bar{P} W_{1}^{\prime \prime}+\bar{C}_{1} \dot{W}_{1}-\bar{K}_{2} W_{1}^{\prime \prime} \int_{0}^{l_{1}} W_{1}^{\prime 2} d x=-\ddot{W}_{B} \\
\ddot{\phi}+\bar{C}_{2} \dot{\phi}-\bar{K}_{t} \phi^{\prime \prime}=0 \\
\ddot{W}_{i}+\bar{K}_{i} W_{i}^{\prime \prime \prime \prime}+\bar{C}_{i} \dot{W}_{i}=-\ddot{W}_{B} \quad(i=3,4)
\end{gathered}
$$


where the coefficients are

$$
\begin{gathered}
\left.()^{\prime}=\frac{\partial}{\partial x}, \quad C^{\cdot}\right)=\frac{\partial}{\partial t}, \quad \bar{K}_{i}=\frac{E I_{y_{i}}}{\bar{m}_{i}}, \quad \bar{C}_{i}=\frac{C_{i}}{\bar{m}_{i}}, \quad \bar{P}=\frac{P}{\bar{m}_{1}} \quad, \quad \bar{K}_{2}=\frac{E A_{1}}{2 \bar{m}_{1} l_{1}} \\
\bar{C}_{2}=\frac{C_{2}}{\rho J_{p}} \quad, \quad \bar{K}_{t}=\frac{G \gamma}{\rho J_{p}}, \quad \gamma=b h^{3}\left[\frac{1}{3}-0.21 \frac{h}{b}\left(1-\frac{h^{4}}{12 b^{4}}\right)\right], \quad(i=1,3,4)
\end{gathered}
$$

\subsection{Boundary and Matching Conditions}

The method used to solve the dynamic behavior of the system is based on the separation of variables, in which a presumed solution is considered as a valid answer to the differential equation. In order to use this method, these assumed functions need to satisfy the essential geometric boundary and matching conditions in the system. One way to find these true shape functions is first solving the equivalent linearized system of equation for each component using separation of variables. Shape functions for each individual component can be achieved by applying certain boundary conditions for free linear vibration. These obtained spatial functions can be used as the basis functions in the nonlinear partial differential equations (PDE). In the next step, the equations of motion for each component is discretized using Galerkin method. After discretization is done, a set of ordinary differential equations is achieved, which can be combined with matching conditions applying at the connection points. Finally, the obtained set of discretized ordinary differential equations (ODE's) is solved and evaluated using the numerical method RungeKutta.

The essential end boundary conditions applied to each individual component of the system as well as the geometrical matching conditions at the connections can be written as follows: 


$$
\begin{gathered}
W_{1}(0)=W_{1}^{\prime}(0)=W_{1}\left(l_{1}\right)=W_{1}^{\prime}\left(l_{1}\right)=W_{1}\left(\frac{l_{1}}{2}\right)=0 \\
\phi(0)=\phi\left(l_{2}\right)=0 \\
W_{i}(0)=W_{i}^{\prime \prime}\left(l_{i}\right)=0 ; \quad(i=3,4) \\
W_{1}^{\prime}\left(\frac{l_{1}}{2}\right)=\phi\left(\frac{l_{2}}{2}\right) ; \quad W_{3}^{\prime}(0)=\phi\left(\frac{l_{2}}{8}\right) ; \quad W_{4}^{\prime}(0)=\phi\left(\frac{7 l_{2}}{8}\right)
\end{gathered}
$$

Note that as shown in Figure 2, the locations of cantilevered arms on the torsional rod are at $l_{2} / 8$ and $7 l_{2} / 8$.

The presumed solution of each component is considered as a linear series of known spatial functions multiplying with time-dependent generalized coordinates:

$$
\begin{array}{cc}
W_{j}(x, t)=\sum_{i=1}^{N_{j}} \psi_{i}^{j}(x) q_{i}^{j}(t), \quad 0 \leq x \leq l_{j} \quad(j=1,3,4) \\
\phi(x, t)=\sum_{i=1}^{N_{2}} \psi_{i}^{2}(x) q_{i}^{2}(t), & 0 \leq x \leq l_{2}
\end{array}
$$

The typical shape functions for a clamped-clamped beam and torsional rod are written as bellow:

$$
\begin{gathered}
\psi_{j}^{1}(x)=\cosh \left(\beta \frac{x}{l_{1}}\right)-\cos \left(\beta_{j} \frac{x}{l_{1}}\right)-\alpha_{j}\left(\sinh \left(\beta_{j} \frac{x}{l_{1}}\right)-\sin \left(\beta_{j} \frac{x}{l_{1}}\right)\right), \\
(j=2,4, \ldots, 2 N) \\
\psi_{j}^{2}(x)=\sin \left(\frac{j \pi x}{l_{2}}\right), \quad(j=1,3, \ldots, 2 N-1)
\end{gathered}
$$


$\alpha_{j}$, and $\beta_{j}$ are the eigenvalues of the clamped-clamped Euler-Bernoulli beam. Note that the geometrical matching conditions at the connections between the central beam and the torsional rod results in considering even modes for the clamped beam and odd modes for the torsional rod. In order to find the shape functions for the cantilever beams, the free undamped vibration of an individual beam with the same boundary conditions is considered (for simplicity the subscript $j=3,4$ is ignored here):

$$
\begin{gathered}
\ddot{W}+\left(\frac{E I_{y}}{\bar{m}}\right) W^{\prime \prime \prime \prime}=0 \\
W(0)=W^{\prime \prime}(0)=W^{\prime \prime}(l)=0, \quad E I_{y} W^{\prime \prime \prime}(l)=M \ddot{W}(l)
\end{gathered}
$$

By considering the typical harmonic motion as the presumed solution for the timedependent coordinate and substituting it into the equation of motion, the following spatialdependent equation is obtained:

$$
\frac{d^{4} \psi(x)}{d x^{4}}-\beta^{4} \psi(x)=0 ; \quad \beta^{4}=\frac{\bar{m} \omega^{2}}{E I_{y}}
$$

The general solution for this equation can be written as:

$$
\psi(x)=A \sin (\beta x)+B \cos (\beta x)+C \sinh (\beta x)+D \cosh (\beta x)
$$

Considering this general solution along with applying all four boundary conditions results in the following relation:

$$
\begin{gathered}
{\left[-\cos (\beta l)+R_{n} \cosh (\beta l)\right]+\frac{M}{\bar{m}} \beta\left[\sin (\beta l)+R_{n} \sinh (\beta l)\right]=0 ;} \\
R_{n}=\frac{\sin (\beta l)}{\sinh (\beta l)}
\end{gathered}
$$


in which $M$ is the proof mass attached to the end of the cantilever beam. Solving the obtained equation results in shape functions for the cantilever beam as bellow:

$$
\psi_{i}(x)=\sin \left(\beta_{n} x\right)+R_{n} \sinh \left(\beta_{n} x\right) ; \quad(n=1,2, \ldots)
$$

\subsection{Discretization and Solving Process}

After finding the linear shape functions for all components, the presumed solution (equations 18,19 ) can be used to discretize the equations of motion using a Galerkin's approach. Applying Galerkin discretization method for each component following by integrating over their domain, the discretized system of equations for each component is achieved as follows:

$$
\begin{gathered}
\sum_{j=1}^{N} m_{i j}^{1} \ddot{q}_{1}^{j}+\sum_{j=1}^{N} c_{i j}^{1} \dot{q}_{1}^{j}+\sum_{j=1}^{N} k_{i j}^{1} q_{1}^{j}+\sum_{j=1}^{N} \sum_{k=1}^{N} \sum_{l=1}^{N} K_{i j k l}^{2} q_{1}^{j} q_{1}^{k} q_{1}^{l}=F_{i}^{1} ; \\
\sum_{j=1}^{N} m_{i j}^{2} \ddot{q}_{2}^{j}+\sum_{j=1}^{N} c_{i j}^{2} \dot{q}_{2}^{j}+\sum_{j=1}^{N} k_{i j}^{2} q_{2}^{j}=0 ; \quad(i=1,2, \ldots, N) \\
\sum_{j=1}^{N} m_{i j}^{n} \ddot{q}_{n}^{j}+\sum_{j=1}^{N} c_{i j}^{n} \dot{q}_{n}^{j}+\sum_{j=1}^{N} k_{i j}^{n} q_{n}^{j}=F_{i}^{n} ; \quad(i=1,2, \ldots, N), \quad(n=3,4)
\end{gathered}
$$

where

$$
\begin{aligned}
& m_{i j}^{n}=\int_{0}^{l_{n}} \psi_{n}^{i} \psi_{n}^{j} d x ; \quad c_{i j}^{n}=\bar{C}_{n} m_{i j}^{n} ; \quad F_{i}^{n}=-\int_{0}^{l_{n}} \ddot{W}_{B} \psi_{n}^{i} d x,(n \neq 2) \\
& k_{i j}^{1}=\bar{K}_{1} \int_{0}^{1} \psi_{1}^{j^{\prime \prime \prime \prime}} \psi_{1}^{i} d x+\bar{P} \int_{0}^{l_{1}} \psi_{1}^{j^{\prime \prime}} \psi_{1}^{i} d x ; K_{i j k l}^{2}=-\bar{K}_{2} \int_{0}^{l_{1}} \psi_{1}^{j^{\prime \prime}} \psi_{1}^{i} d x \int_{0}^{l_{1}} \psi_{1}^{k^{\prime}} \psi_{1}^{l^{\prime}} d x
\end{aligned}
$$


$k_{i j}^{2}=-\bar{K}_{t} \int_{0}^{l_{2}} \psi_{2}^{j^{\prime \prime}} \psi_{2}^{i} d x ; k_{i j}^{3}=\int_{0}^{l_{3}} \psi_{3}^{j^{\prime \prime \prime \prime}} \psi_{3}^{i} d x ; k_{i j}^{4}=\int_{0}^{l_{4}} \psi_{4}^{j^{\prime \prime \prime \prime \prime}} \psi_{4}^{i} d x ; \quad(n=1,2,3,4)$

Substituting presumed solutions into the essential matching conditions (equation 17) at the connecting points results in:

$$
\begin{aligned}
& \sum_{i=1}^{N} q_{1}^{i} \psi_{1}^{i}\left(\frac{l_{1}}{2}\right)=\sum_{i=1}^{N} q_{2}^{i} \psi_{2}^{i}\left(\frac{l_{2}}{2}\right) \\
& \sum_{i=1}^{N} q_{3}^{i} \psi_{3}^{i^{\prime}}(0)=\sum_{i=1}^{N} q_{2}^{i} \psi_{2}^{i}\left(\frac{l_{2}}{8}\right) \\
& \sum_{i=1}^{N} q_{4}^{i} \psi_{4}^{i^{\prime}}(0)=\sum_{i=1}^{N} q_{2}^{i} \psi_{2}^{i}\left(\frac{7 l_{2}}{8}\right)
\end{aligned}
$$

For simplicity, the same number of modes is considered for each part in the calculation process. There are three matching conditions in total, and therefore three additional relations between generalized coordinates, which results in the dependency of three of them to the rest of generalized coordinates. Applying equations (31-33) reduces the numbers of the degrees of freedom for the whole system from $4 N$ to $4 N-3$ (in this case $N$ modes are considered for each component which makes $4 N$ modes for the whole system in total). To do so, 3 constrained (dependent) coordinates needs to be introduced. By considering $q_{2}^{N}, q_{3}^{N}$, and $q_{4}^{N}$ as the 3 constrained generalized coordinates, the relation between the new set of variables (unconstrained coordinates) and the original one, which is called transformation matrix $[B]$, can be written by rearranging the obtained relations and eliminating these three dependent coordinates from the whole system:

$$
\underline{q}_{4 N \times 1}=[B] \underline{\bar{q}}_{(4 N-3) \times 1}
$$


where $q$ and $\bar{q}$ are the vector of total and reduced (independent) generalized coordinate system respectively. By substituting the obtained transformation into the set of above ordinary differential equations and solving them numerically, the independent generalized coordinates and therefore the dynamic equation for the whole system can be written as:

$$
[\bar{M}] \underline{\bar{q}}+[\bar{C}] \underline{\dot{q}}+[\bar{K}] \underline{\bar{q}}+[B] \underline{f_{N T}}=[B] \underline{F}
$$

where

$$
[\bar{M}]=[B]^{T}[M][B] ; \quad[\bar{C}]=[B]^{T}[C][B] ; \quad[\bar{K}]=[B]^{T}[K][B]
$$

Considering nonlinearity existing in the obtained equation system, the best and straightforward way to solve the equation of motion is using a numerical method. The numerical method utilized here is Runge-Kutta, which is an iterative evolutionary method suitable for solving ordinary differential equations (ODE's). In order to solve a higher order differential equation with Runge-Kutta, the system of equations need to be written in the first-derivative form. To do so, a set of new variables are defined as bellow:

$$
\left\{\begin{array}{l}
\underline{\bar{q}}=\underline{X}_{1} \\
\dot{\bar{q}}=\underline{X}_{2}
\end{array}\right.
$$

in which both displacement and velocity vectors are taken as state variables. By considering this set of new variables, the obtained nonlinear equation of motion can be rewritten in a state space form:

$$
\left\{\begin{array}{c}
\dot{X}_{1}=\underline{X}_{2} \\
\underline{X}_{2}=f\left(\underline{X_{1}}, \underline{X_{2}}\right)
\end{array}\right.
$$

After solving equation (38) numerically, the constrained generalized coordinates for the displacement of each component are found and by using transformation matrix $[B]$, the 
generalized coordinate vector is obtained. Substituting this vector into the presumed solution (equations 18, 19), the deflection response for each component of the system can be developed.

\subsection{Experimental Setup}

In order to verify the model proposed in this section, an experimental setup replicating the structure was considered. Figure 4 shows the setup of the experiment for capturing the central beam deflection response. To do so, a custom-built shaker table [78] was used to test the device under harmonic excitation. The shaker system consisted of an anchor platform mounted on a $42 \mathrm{~W}$ speaker driven using an audio amplifier. An Arduino Nano (V3.0) was used to read the accelerometer and send its value to a LabVIEW program [79]. The testing device is made of Acrylonitrile-Butadiene-Styrene (ABS) using a GEEETech 3D printer with the dimensions listed in Table 1. As it is shown in Figure 4(b), the device is fixed on the shaker table at both ends of the main beam and torsional rod and the axial load is exerted by changing the distance between two longitudinal fixed ends. The displacement at the mid-point of the main beam was measured with anoptoNCDT 1401 measuring sensor and the output results of the laser sensor were analyzed using an Infiniium Digital Storage Oscilloscope. 
Table 1. Dimensions and physical parameters of the experimental setup.

\begin{tabular}{|l|c|c|c|}
\hline Component & Main beam & Torsional rod & Cantilevers \\
\hline Module of Elasticity $(\mathrm{GPa})$ & 2.3 & 2.3 & 2.3 \\
\hline Density $\left(\mathrm{kg} / \mathrm{m}^{3}\right)$ & 1040 & 1040 & 1040 \\
\hline Length $(\mathrm{mm})$ & 86 & 50 & 63 \\
\hline Thickness $(\mathrm{mm})$ & 5 & 5 & 5 \\
\hline Height $(\mathrm{mm})$ & 1.3 & 1.8 & 1.8 \\
\hline Damping coefficient $(\mathrm{N} . \mathrm{s} / \mathrm{m})$ & 0.1 & 0.1 & 0.1 \\
\hline Tip mass $(\mathrm{gr})$ & - & - & 2.36 \\
\hline Poisson's ratio & 0.35 & 0.35 & 0.35 \\
\hline
\end{tabular}



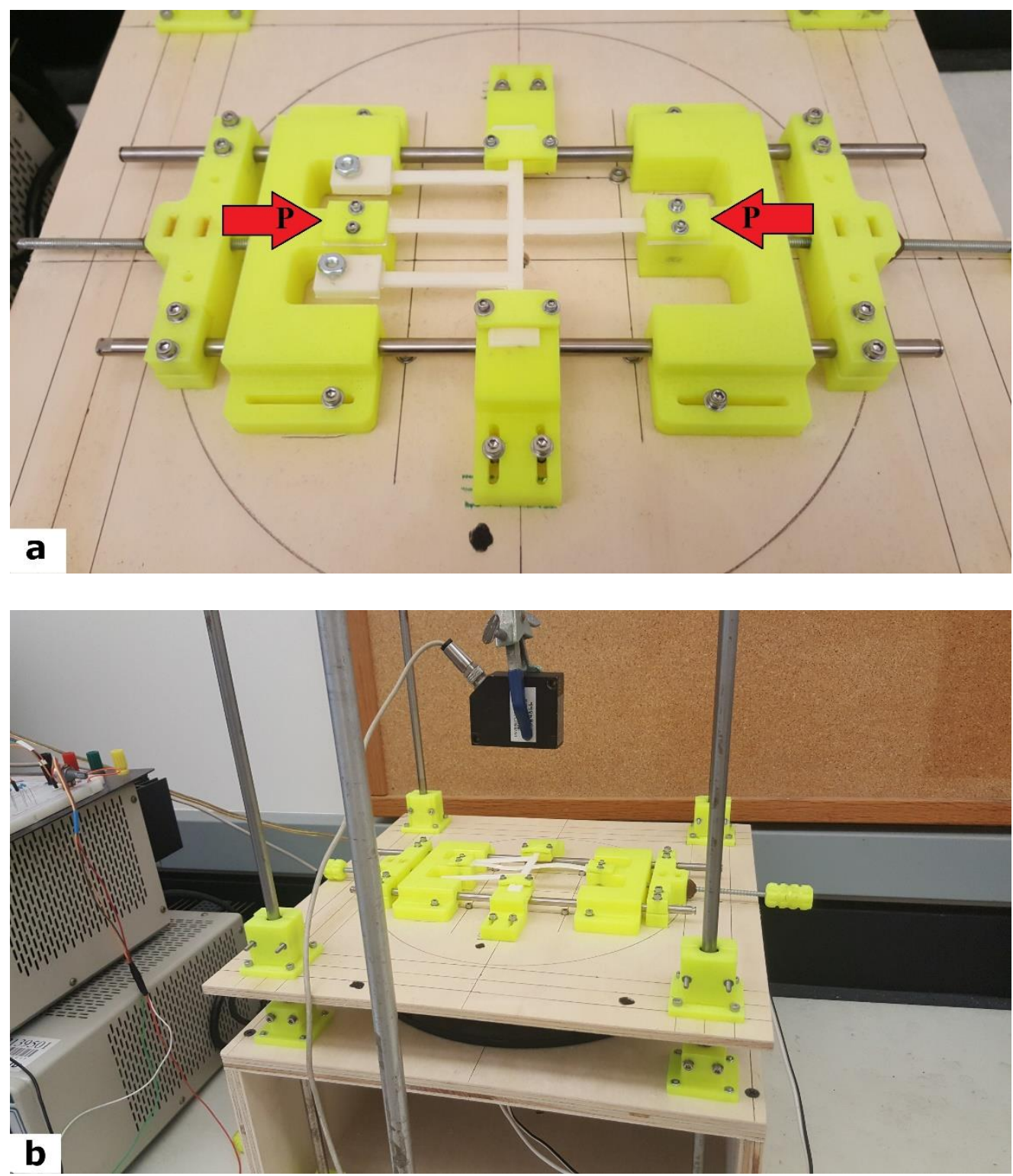

Figure 4. The experimental setup: (a) custom-built shaker table with the optoNCDT 1401 measuring sensor. (b) testing device mounted on the shaker table.

The dynamic response of the main beam is analyzed by the explained experimental setup under harmonic excitation and compared with their corresponding modeling results. In this examination, three situations of buckling levels are considered for the experimental setup: unbuckled, buckled and highly buckled cases. For modeling, three numbers of modes are 
considered for each component in the calculation, which results in twelve total degrees of freedom for the whole system.

\subsection{Results and Discussion}

Figure 5 shows the amplitude-frequency response at the midpoint of the central beam for both experimental and modeling results. The amplitude considered in this plot is measured with respect to the base vibration. As can be seen from the figure, the relative amplitude of the beam in the experimental data is relatively small and qualitatively in agreement with the modeling results except for frequencies at around $25 \mathrm{~Hz}$, which is close to the first natural frequency of the cantilever arms (the first natural frequency of the cantilever arm is theoretically calculated at $25.86 \mathrm{~Hz}$ ). The reason for this inconsistency can be explained due to the mode shapes considered in the modeling formulation, which are not the exact shape functions of the coupled structure and just satisfy the geometrical boundary conditions and consequently, cannot appropriately describe the force transferring between two connected parts. Furthermore, the observed results show that the first natural frequency of the system is dominated by the cantilever arms, and the system encounters a pretty large motion due to their resonance behaviors, which in turn alleviates snap-through motion in the main beam. Experimental observation has shown that even for higher buckling levels in the system, which can guarantee the existence of two stable states, reaching to the highenergy orbit of the device is possible at relatively low frequency range and poor excitation amplitudes, which is one of the main issues associated with bistable energy harvesters, by appropriately setting the physical parameters of the cantilever arms and torsional rod based on the system working conditions. 


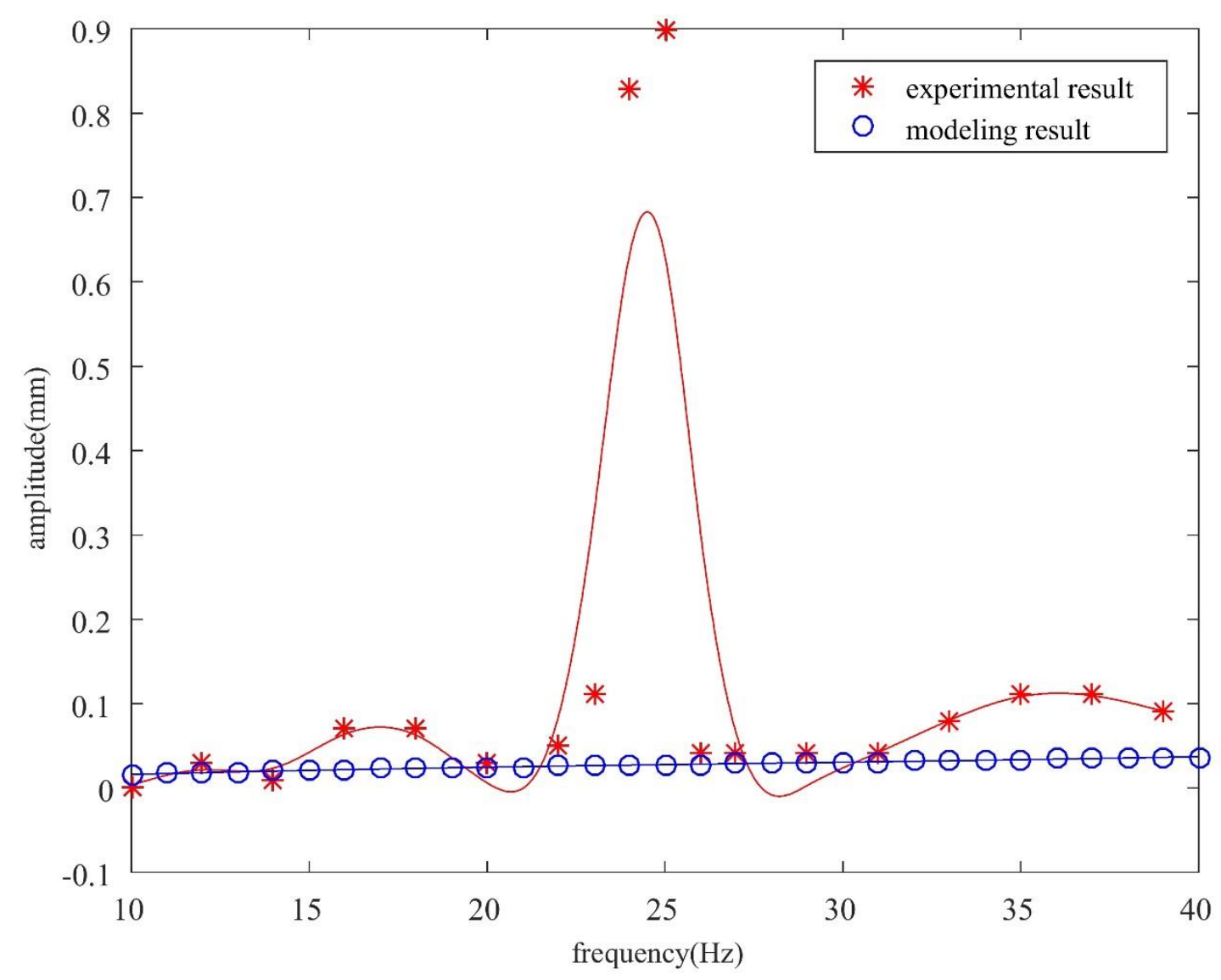

Figure 5. Amplitude-frequency response of the midpoint of the central beam for both modeling and experimental results.

In Figure 6, the variation of the central beam vibrational amplitude vs. the buckling level is experimentally examined at $25 \mathrm{~Hz}$ excitation frequency. The buckling load is swept forward by reducing the distance between two clamped ends of the main beam step by step. Note that this is just the experimental results and the base amplitude is set high enough for snap-through motion occurs in the buckled beam. As shown in the figure, the plot is divided into three different regimes: unbuckled, buckled and highly buckled conditions. The relative vibration amplitude of the main beam significantly increases when buckling happens at $\Delta=5 \mathrm{~mm}$ due to the hardening behavior of the system. Theoretically, this is 
just a transient state and after the beam is buckled, it will stay at one of its stable positions. However, due to the large vibrational energy transferring by the cantilever arms to the main beam, the system can stay in the high-energy loop for a longer time and a wider range of buckling loads, which results in significant strains in the main beam and eventually helps the device harvesting more output power. Moreover, for highly buckled case, in which the compressive load applied to the central beam ends is pretty large, only local vibration occurs in the system.

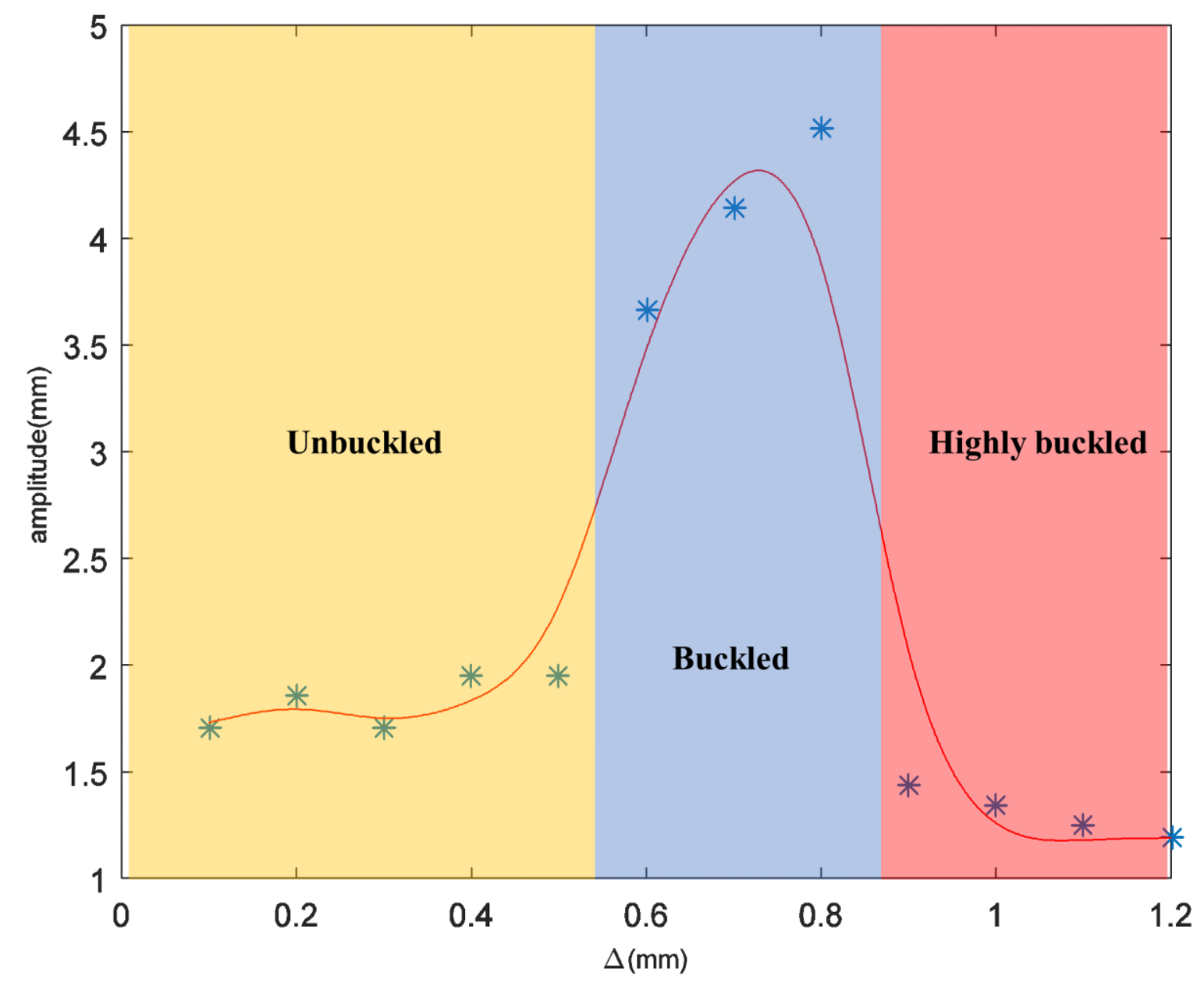

Figure 6. Change of vibration amplitude of the buckled main beam vs. buckling level for $25 \mathrm{~Hz}$ exciting frequency. 
As shown in this chapter, a theoretical model of the system was developed by dividing it into its individual components and deriving the discretized equations of motion for each part using a Galerkin's approach. To do so, a summation of shape functions which can satisfy only the essential geometrical boundary conditions along with their corresponding time-dependent generalized coordinates were considered as the general solution. After obtaining the ordinary differential equations for the system, the geometrical matching conditions were written which results in finding transformation matrix relating the general and independent set of generalized coordinates for the whole system.

In the next part, an experimental setup was considered to compare the results with the proposed model. It was shown that the connected cantilever arms can play an important role in helping the main beam switching between its two stable states. This effect can be significantly highlighted at frequencies close to the first natural frequency of the system, which is dominated by the cantilever arms. However, the proposed model fails to show this highlighted effect of the experimental device, which is essential for the studied structure to work as a low-frequency bistable energy harvester and proves that capturing accurate dynamic behavior of the system requires a more hybrid approach. In the next chapter, a new approach will be introduced to resolve this issue, which results in a more accurate model and consequently leads to a better prediction of the system dynamic behavior. 


\section{COMPONENT COUPLING METHOD TO MODEL THE BISTABLE STRUCTURE}

As seen from the previous chapter, the proposed model to analyze the dynamic behavior of the coupled component failed to follow the expected behavior observed in the experimental setup. The main reason for this issue was identified due to the types of shape functions as well as the coupling method chosen to solve the problem, which only satisfy the geometrical boundary conditions at the connection points and were not able to show the force/moment transfer between different components of the structure, which is the main reason of bisable motion occurrence in the low-frequency range. To address the mentioned issue, a new coupling approach is introduced in this chapter to accurately analyze both the static and dynamic behavior of the buckled system.

\subsection{Approximate Coupling Methods}

When a machine or structure is physically coupled, the process of joining different parts makes them work as a single coupled system. This behavior comes from the connections of the structure, which make the system has a united kinematic characteristic, which can also be seen in a multi-degree of freedom systems. There are some well-known approximate methods introduced for the modal analysis of such coupled structures, two of the most important which are briefly discussed in this chapter.

\subsubsection{Lagrange Multipliers}


In this method, the forces and moment that make the structure connected are considered as constrained conditions or constrained forces, which are commonly called reactions. First, a series of trial functions (usually polynomial functions) are chosen for each component of the system. These functions must satisfy the essential or geometrical boundary conditions existing at the joints. These constraint conditions must be satisfied to make the system components move consistently. To do so, the discretized form of the equation for each part of the system is developed, which leads to the uncoupled system of equation for the whole structure. The next step requires to write the constraint conditions (boundary conditions at the connections) as a separate relation. Finally, solving the whole uncoupled system constrained to the reaction conditions leads to the approximate solutions for the coupled structure [80].

\subsubsection{Component Mode Synthesis (CMS)}

Another general method used to solve coupled systems is Component Mode Synthesis (CMS). In this approach, first, the components of the structures are decoupled and the type of connection at each individual joint gets clarified. Then some additional constraints are added to each component to build types of structures, which shape functions are known and can be developed easily. After that, some shape functions introduced as constrained modes. These constrained modes can be a set of polynomial functions, in which all end boundaries are set to be zero (fixed conditions) except for the ones which are able to move

at the connections in the coupled structures. By forming these functions, the general solution for each component can be written as a combination of the fixed and constrained modes, by which the discretized equation of motion can be shaped for the whole system. The last thing to do is choosing some generalized coordinates from the constrained modes 
as the independent coordinate system to form the relations existing at the connections of the structure and consequently finding the transformation matrix relating the total and independent generalized coordinate system. Applying this transformation by use of Lagrange Multipliers method would lead to the equation of motion for the coupled system. Note that CMS is also an approximate method by considering the fact that the types of shape functions considered in this approach are not exactly the true shape functions of the system $[80,81]$.

\subsection{Proposed Component Coupling Approach}

As shown in the previous section, the method to analyze the coupled structures are based on dividing the whole system into its component in a way that all boundary conditions at the connections can be satisfied. Since these methods only deliver approximate solutions for linear systems, we intend to solve the nonlinear system by finding the exact shape functions of the coupled structure. To do so, the linearized coupled system needs to be solved via an accurate approach. The basic idea of this approach is the same as the method used to do the dynamic analysis of a simple beam combined of two different material properties as described in Figure 7. To solve such structure, the beam is divided into two individual beams with unique material properties, for which the exact general shape functions can be composed. These general functions include some unknown constant coefficients, which can be found from equating the boundary conditions of each part at the connection. 


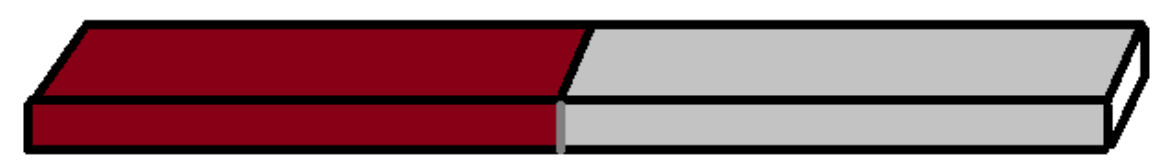

(EI) 2

(EI) 1

Figure 7. A schematic view of a single beam with different material properties.

The general approach employed in this chapter is to divide the studied coupled structure into its individual components and enforce all geometrical and force-moment boundary conditions at the connection points. First, the quasi-static buckled state is determined, about which a linearized dynamic model is generated. After developing the linearized shape functions, Galerkin's method along with Lagrange multipliers are implemented to discretize the nonlinear partial differential equations of the coupled system, resulting in a reduced-order set of ordinary differential equations. This dynamic model is used to predict amplitude-frequency response and snap-through regimes with respect to buckling load over a range of viable energy harvesting conditions of excitation frequency and base vibration. Both finite element analysis (FEA) and experimental testing are used to validate the model based on which general behavior predictions made.

\subsection{Formulation and Solving Procedure}

In order to model accurately the dynamic behavior of the described coupled system, all boundary and matching conditions at the connection points of each individual component need to be satisfied. To do so, the coupled system is divided into eight separate parts as shown in Figure 8, where the central beam and the torsional rod are each divided into two and four parts, respectively. 


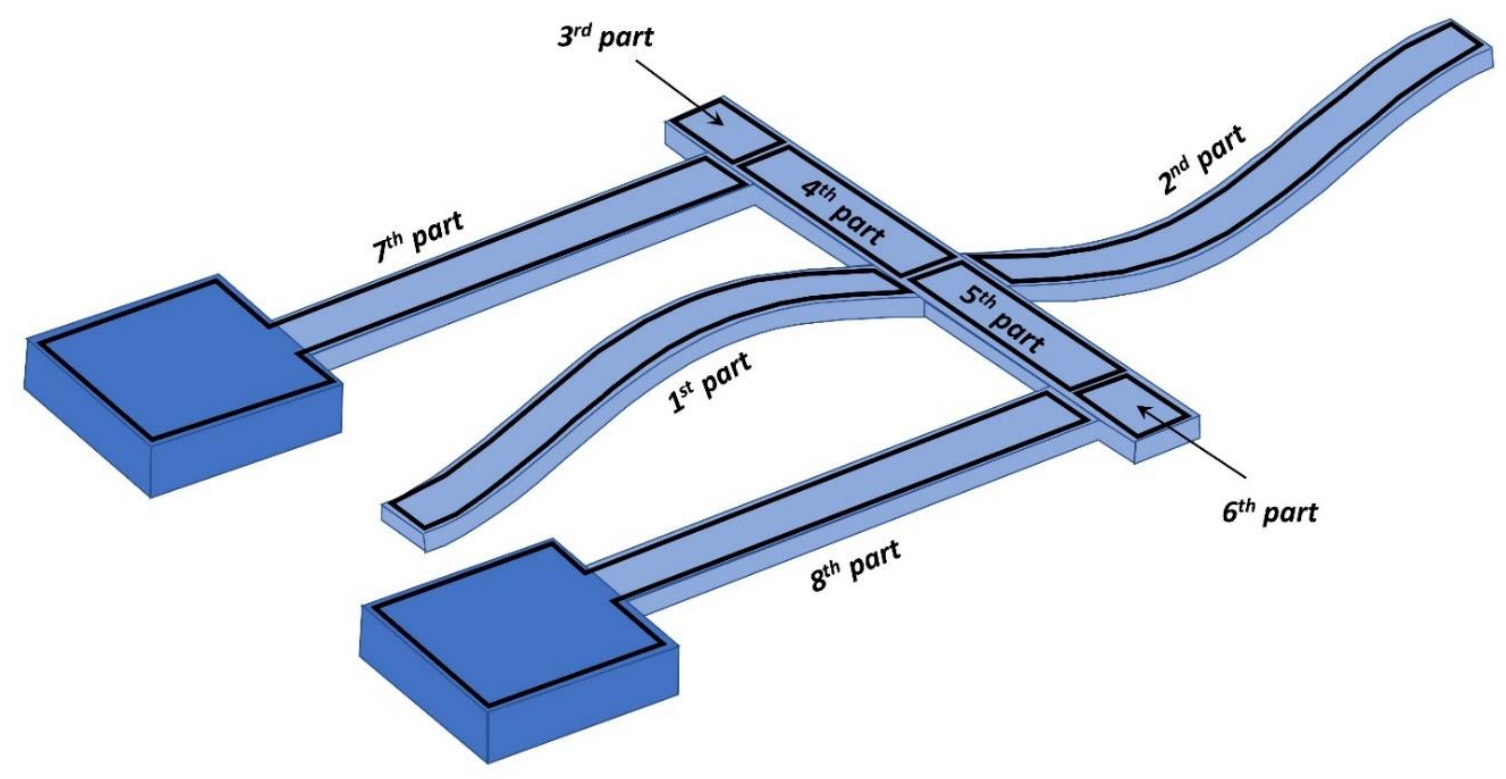

Figure 8. The coupled structure divided into eight separate parts.

Based on the division and dimensions shown in Figures 2 and 8, equations (11-13) can be restated for each individual part domain as follows:

$$
\begin{gathered}
\ddot{W}_{1}+\bar{K}_{1} W_{1}^{\prime \prime \prime \prime}+\bar{P} W_{1}^{\prime \prime}+\bar{C}_{1} \dot{W}_{1}-\frac{\bar{K}_{2}}{2} W_{1}^{\prime \prime}\left(\int_{0}^{\frac{l_{1}}{2}} W_{1}^{\prime 2} d x\right)=-\ddot{W}_{B}, \quad\left(0<x<\frac{l_{1}}{2}\right) \\
\ddot{W}_{2}+\bar{K}_{1} W_{2}^{\prime \prime \prime \prime}+\bar{P} W_{2}^{\prime \prime}+\bar{C}_{1} \dot{W}_{2}-\frac{\bar{K}_{2}}{2} W_{2}^{\prime \prime}\left(\int_{\frac{l_{1}}{2}}^{l_{1}}{W_{2}^{\prime}}^{2} d x\right)=-\ddot{W}_{B}, \quad\left(\frac{l_{1}}{2}<x<l_{1}\right) \\
\ddot{\phi}_{3}+\bar{C}_{2} \dot{\phi}_{3}-\bar{K}_{t} \phi_{3}^{\prime \prime}=0, \quad\left(0<x<\frac{l_{2}}{8}\right) \\
\ddot{\phi_{4}}+\bar{C}_{2} \dot{\phi}_{4}-\bar{K}_{t} \phi_{4}^{\prime \prime}=0, \quad\left(\frac{l_{2}}{8}<x<\frac{l_{2}}{2}\right)
\end{gathered}
$$




$$
\begin{array}{ll}
\ddot{\phi}_{5}+\bar{C}_{2} \dot{\phi}_{5}-\bar{K}_{t} \phi_{5}^{\prime \prime}=0, \quad\left(\frac{l_{2}}{2}<x<\frac{7 l_{2}}{8}\right) \\
\ddot{\phi}_{6}+\bar{C}_{2} \dot{\phi}_{6}-\bar{K}_{t} \phi_{6}^{\prime \prime}=0, \quad\left(\frac{7 l_{2}}{8}<x<l_{2}\right) \\
\ddot{W}_{7}+\bar{K}_{3} W_{7}^{\prime \prime \prime \prime}+\bar{C}_{3} \dot{W}_{7}=-\ddot{W}_{B}, \quad\left(0<x<l_{3}\right) \\
\ddot{W}_{8}+\bar{K}_{4} W_{8}^{\prime \prime \prime \prime}+\bar{C}_{4} \dot{W}_{8}=-\ddot{W}_{B}, \quad\left(0<x<l_{4}\right)
\end{array}
$$

\subsubsection{Solving the Static System}

To find the buckled configuration of the system and the critical buckling load required to make the system bistable, the static behavior of the structure is first analyzed. By dropping the dynamic, damping, and base vibration terms, the static equations of the system are obtained as follows:

$$
\begin{gathered}
\bar{K}_{1} W_{1}^{\prime \prime \prime \prime}+\bar{P} W_{1}^{\prime \prime}-\frac{\bar{K}_{2}}{2} W_{1}^{\prime \prime}\left(\int_{0}^{\frac{l_{1}}{2}} W_{1}^{\prime 2} d x\right)=0 \\
\bar{K}_{1} W_{2}^{\prime \prime \prime \prime}+\bar{P} W_{2}^{\prime \prime}-\frac{\bar{K}_{2}}{2} W_{2}^{\prime \prime}\left(\int_{\frac{l_{1}}{2}}^{l_{1}} W_{2}^{\prime 2} d x\right)=0 \\
\phi_{i}^{\prime \prime}=0 \quad(i=3,4,5,6) \\
W_{i}^{\prime \prime \prime \prime}=0 \quad(i=7,8)
\end{gathered}
$$

Based on the above static equations, the general solutions for parts 3-8, which are considered as linear parts, are polynomial functions:

$$
\phi_{i}^{S}=A_{i}^{S}+B_{i}^{S} x, \quad(i=3,4,5,6)
$$




$$
W_{i}^{s}=A_{i}^{s}+B_{i}^{s} x+C_{i}^{s} x^{2}+D_{i}^{s} x^{3}, \quad(i=7,8)
$$

Since parts 1 and 2 include nonlinear terms, several steps need to be taken in order to find their general static solutions. Equations (47) and (48) can be rewritten as follows:

$$
\begin{gathered}
\bar{K}_{1} W_{1}^{\prime \prime \prime \prime}+\left(\bar{P}-\bar{\Gamma}_{1}\right) W_{1}^{\prime \prime}=0 ; \quad\left(\bar{\Gamma}_{1}=\frac{\bar{K}_{2}}{2} \int_{0}^{\frac{l_{1}}{2}} W_{1}^{\prime 2} d x\right) \\
\bar{K}_{1} W_{2}^{\prime \prime \prime \prime}+\left(\bar{P}-\bar{\Gamma}_{2}\right) W_{2}^{\prime \prime}=0 ; \quad\left(\bar{\Gamma}_{2}=\frac{\bar{K}_{2}}{2} \int_{\frac{l_{1}}{2}}^{l_{1}}{W_{2}^{\prime 2}}^{2} d x\right)
\end{gathered}
$$

Due to the geometrical symmetry existing in the static shape of the main beam, $\bar{\Gamma}_{1}$ and $\bar{\Gamma}_{2}$ are equal, and for simplicity are considered as just $\bar{\Gamma}$. Based on this assumption, the general solutions for static differential equations of parts 1 and 2 are obtained as follows:

$$
\begin{aligned}
& W_{1}^{s}=A_{1}^{s} \sin \left(\beta_{s} x\right)+B_{1}^{s} \cos \left(\beta_{s} x\right)+C_{1}^{s}+D_{1}^{s} x \\
& W_{2}^{s}=A_{2}^{s} \sin \left(\beta_{s} x\right)+B_{2}^{s} \cos \left(\beta_{s} x\right)+C_{2}^{s}+D_{2}^{s} x
\end{aligned}
$$

where the superscript " $s$ " refers to the static terms and the coefficient $\beta_{s}$ is prescribed as

$$
\beta_{s}{ }^{2}=\frac{\bar{P}-\bar{\Gamma}}{\overline{K_{1}}}
$$

To find the unknown coefficients of the static solutions and the critical buckling load that determines the exact static shape of the main buckled beam, boundary and matching conditions of the system need to be appropriately applied on the obtained general solutions. For this specific problem, boundary conditions can be divided into two different types, end boundaries and matching conditions. End boundaries constitute support conditions 
(interactions with the base support structure), while matching conditions at the connection points joining discrete components parts must be satisfied to ensure continuity in behavior of the coupled system.

I- End Boundaries:

The following relations represent the 14 end boundaries for different parts of the system:

$$
\begin{aligned}
& \left\{\begin{array}{lll}
W_{1}(0)=0 ; & W_{1}^{\prime}(0)=0 ; & W_{1}\left(\frac{l_{1}}{2}\right)=0 \\
W_{2}\left(l_{1}\right)=0 ; & W_{2}^{\prime}\left(l_{1}\right)=0 ; & W_{2}\left(\frac{l_{1}}{2}\right)=0
\end{array}\right. \\
& W_{1}^{S}(x)=A_{1}^{s}\left\{\sin \left(\beta_{s} x\right)-\beta_{s} x-R_{1}^{S}\left[\cos \left(\beta_{s} x\right)-1\right]\right\} \\
& W_{2}^{S}(x)=A_{2}^{S}\left\{\sin \left(\beta_{s} x\right)+R_{2 B}^{S} \cos \left(\beta_{s} x\right)+R_{2 C}^{S}+R_{2 D}^{S} x\right\}
\end{aligned}
$$

where

$$
\begin{aligned}
& \left\{\begin{aligned}
R_{2 B}^{S}= & \frac{\beta_{s} L_{1} \cos \left(\beta_{s} L_{1}\right)+2 \sin \left(\frac{\beta_{S} L_{1}}{2}\right)-2 \sin \left(\beta_{s} L_{1}\right)}{-2 \cos \left(\frac{\beta_{S} L_{1}}{2}\right)+2 \cos \left(\beta_{s} L_{1}\right)+\beta_{s} L_{1} \sin \left(\beta_{s} L_{1}\right)} \\
R_{2 C}^{S}= & \frac{\beta_{S} L_{1}-2 \beta_{S} L_{1} \cos \left(\frac{\beta_{S} L_{1}}{2}\right)+2 \sin \left(\frac{\beta_{S} L_{1}}{2}\right)}{-2 \cos \left(\frac{\beta_{S} L_{1}}{2}\right)+2 \cos \left(\beta_{S} L_{1}\right)+\beta_{s} L_{1} \sin \left(\beta_{S} L_{1}\right)} \\
R_{2 D}^{S}= & \frac{-4 \beta_{S} \sin ^{2}\left(\frac{\beta_{S} L_{1}}{4}\right)}{-2 \cos \left(\frac{\beta_{S} L_{1}}{2}\right)+2 \cos \left(\beta_{S} L_{1}\right)+\beta_{S} L_{1} \sin \left(\beta_{S} L_{1}\right)}
\end{aligned}\right. \\
& \phi_{3}(0)=0 \quad \stackrel{\text { yields }}{\longrightarrow} \quad \phi_{3}^{s}(x)=A_{3}^{s} x \\
& \phi_{6}\left(L_{2}\right)=0 \quad \stackrel{\text { yields }}{\longrightarrow} \quad \phi_{6}^{S}(x)=A_{6}^{s}\left(-L_{2}+x\right)
\end{aligned}
$$




$$
\left\{\begin{array} { c } 
{ W _ { i } ( 0 ) = 0 } \\
{ W _ { i } ^ { \prime \prime } ( L _ { 3 } ) = 0 } \\
{ W _ { i } ^ { \prime \prime \prime } ( L _ { 3 } ) = 0 }
\end{array} \quad ( i = 7 , 8 ) \quad \stackrel { \text { yields } } { \longrightarrow } \quad \left\{\begin{array}{l}
W_{7}^{S}(x)=A_{7}^{S} x \\
W_{8}^{S}(x)=A_{8}^{S} x
\end{array}\right.\right.
$$

\section{Matching Conditions:}

As shown in the Figure 9, in total there are 3 connections in the system, each has its own geometrical and force-moment matching conditions and these conditions need to be satisfied to get the response for the whole system.
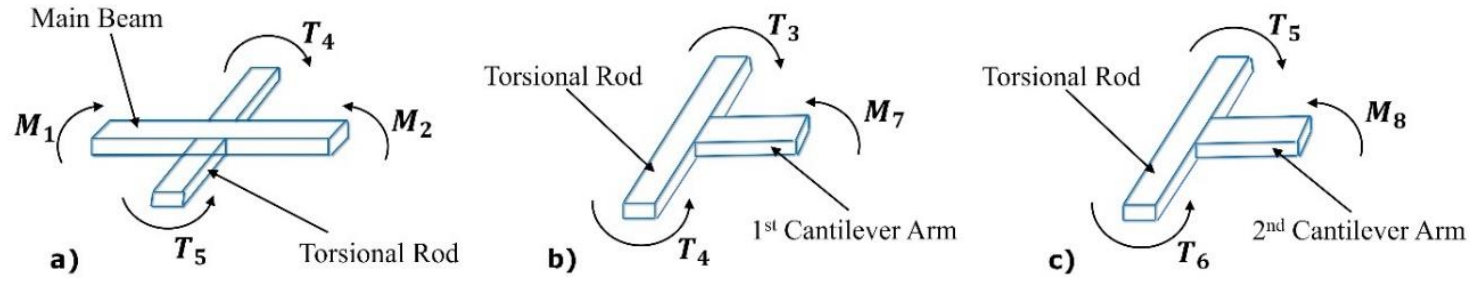

Figure 9. Schematic view of three connections in the system: a) main beam-torsional rod.

b) torsional rod-first cantilever arm. c) torsional rod-second cantilever arm.

For the connection between the buckled beam and torsional rod, it is found:

$$
\begin{gathered}
W_{1}^{\prime}\left(\frac{l_{1}}{2}\right)=W_{2}^{\prime}\left(\frac{l_{1}}{2}\right)=\phi_{4}\left(\frac{l_{2}}{2}\right)=\phi_{5}\left(\frac{l_{2}}{2}\right) \\
(E I)_{1}\left[W_{1}^{\prime \prime}\left(\frac{l_{1}}{2}\right)-W_{2}^{\prime \prime}\left(\frac{l_{1}}{2}\right)\right]+G \gamma\left[\phi_{4}^{\prime}\left(\frac{l_{2}}{2}\right)-\phi_{5}^{\prime}\left(\frac{l_{2}}{2}\right)\right]=0
\end{gathered}
$$

For the connection between the torsional rod and $1^{\text {st }}$ cantilevered arm, it is found:

$$
\begin{gathered}
\phi_{3}\left(\frac{l_{2}}{8}\right)=\phi_{4}\left(\frac{l_{2}}{8}\right)=W_{7}^{\prime}(0) \\
(E I)_{3} W_{7}^{\prime \prime}(0)+G \gamma\left[\phi_{4}^{\prime}\left(\frac{l_{2}}{8}\right)-\phi_{3}^{\prime}\left(\frac{l_{2}}{8}\right)\right]=0
\end{gathered}
$$

For the connection between the torsional rod and $2^{\text {nd }}$ cantilevered arm, it is found: 


$$
\begin{gathered}
\phi_{5}\left(\frac{7 l_{2}}{8}\right)=\phi_{6}\left(\frac{7 l_{2}}{8}\right)=W_{8}^{\prime}(0) \\
(E I)_{3} W_{8}^{\prime \prime}(0)+G \gamma\left[\phi_{6}^{\prime}\left(\frac{7 l_{2}}{8}\right)-\phi_{5}^{\prime}\left(\frac{7 l_{2}}{8}\right)\right]=0
\end{gathered}
$$

As shown in equations (63-68), there are 10 matching conditions, which is equal to the number of remaining unknown constants after applying all of the end conditions. These relations can be written in a matrix-form as follows:

$$
\left[B^{s}\right]\left\{A^{s}\right\}=\{0\}
$$

where the $10 \times 10$ matrix is the static transformation matrix and vector $\left\{A^{S}\right\}$ is

$$
\left\{A^{S}\right\}=\left\{A_{1}^{S}, A_{2}^{S}, A_{3}^{S}, A_{4}^{S}, B_{4}^{S}, A_{5}^{S}, B_{5}^{S}, A_{6}^{S}, A_{7}^{S}, A_{8}^{S}\right\}^{T}
$$

By forming $\operatorname{Det}\left[B^{S}\right]=0$, which gives us the non-zero solutions of vector $\left\{A^{S}\right\}$, the static eigenvalue $\beta_{s}$ and consequently the critical buckling load $P_{c r}=\bar{K}_{1} \beta_{s}^{2}$ will be obtained for a given geometrical and physical parameters of the system. Since there are 10 conditions with 11 unknowns, it should be noted that a certain value of applied buckling load $P$ is required as an initial condition in order to find the eleventh unknown and the exact postbuckled static shape of the whole system from equation (57). After finding the static buckled shape of the system, the next step is finding the dynamic mode shapes about the buckled case, which is obtained by solving the linearized system. These linear mode shapes are then used to discretize the nonlinear equations using Galerkin's method to aid in finding the solution of the nonlinear system behavior.

\subsubsection{Dynamic Analysis of Linearized Buckled System}

The total dynamic solution of each part of the system can be formed as the summation of static and dynamic parts as follows: 


$$
\begin{array}{ll}
W_{i}(x, t)=W_{i}^{s}(x)+V_{i}(x, t), & (i=1,2,7,8) \\
\phi_{i}(x, t)=\phi_{i}^{s}(x)+f_{i}(x, t), & (i=3,4,5,6)
\end{array}
$$

Substituting the above presumed solutions into the dynamic equations of motion (39-46) yields the total dynamic equations of the nonlinear buckled system.

$$
\begin{aligned}
& \ddot{V}_{1}+\bar{K}_{1} V_{1}^{\prime \prime \prime \prime}+\left[\bar{P}-\bar{K}_{2} \int_{0}^{\frac{L_{1}}{2}} W_{1}^{s^{\prime 2}} d x\right] V_{1}^{\prime \prime}-2 \bar{K}_{2} W_{1}^{s^{\prime \prime}} \int_{0}^{\frac{L_{1}}{2}} W_{1}^{s^{\prime}} V_{1}^{\prime} d x \\
& -\bar{K}_{2} W_{1}^{s^{\prime \prime}} \int_{0}^{\frac{L_{1}}{2}} V_{1}^{\prime 2} d x-\bar{K}_{2} V_{1}^{\prime \prime} \int_{0}^{\frac{L_{1}}{2}}{V_{1}^{\prime 2}}^{2} d x-2 \bar{K}_{2} V_{1}^{\prime \prime} \int_{0}^{\frac{L_{1}}{2}} W_{1}^{s^{\prime}} V_{1}^{\prime} d x \\
& +\bar{C}_{1} \dot{V}_{1}=-\ddot{W}_{B}, \quad\left(0<x<\frac{L_{1}}{2}\right) \\
& \ddot{V}_{2}+\bar{K}_{1} V_{2}^{\prime \prime \prime \prime}+\left[\bar{P}-\bar{K}_{2} \int_{\frac{L_{1}}{2}}^{L_{1}} W_{2}^{s^{\prime 2}} d x\right] V_{2}^{\prime \prime}-2 \bar{K}_{2} W_{2}^{s^{\prime \prime}} \int_{\frac{L_{1}}{2}}^{L_{1}} W_{2}^{s^{\prime}} V_{2}^{\prime} d x \\
& -\bar{K}_{2} W_{2}^{s^{\prime \prime}} \int_{\frac{L_{1}}{2}}^{L_{1}} V_{2}^{\prime 2} d x-\bar{K}_{2} V_{2}^{\prime \prime} \int_{\frac{L_{1}}{2}}^{L_{1}} V_{2}^{\prime 2} d x-2 \bar{K}_{2} V_{2}^{\prime \prime} \int_{\frac{L_{1}}{2}}^{L_{1}} W_{2}^{s^{\prime}} V_{2}^{\prime} d x \\
& +\bar{C}_{1} \dot{V}_{2}=-\ddot{W}_{B}, \quad\left(\frac{L_{1}}{2}<x<L_{1}\right) \\
& \ddot{f}_{3}+\bar{C}_{2} \dot{f}_{3}-\bar{K}_{t} f_{3}^{\prime \prime}=0, \quad\left(0<x<\frac{L_{2}}{8}\right) \\
& \ddot{f}_{4}+\bar{C}_{2} \dot{f}_{4}-\bar{K}_{t} f_{4}^{\prime \prime}=0, \quad\left(\frac{L_{2}}{8}<x<\frac{L_{2}}{2}\right) \\
& \ddot{f}_{5}+\bar{C}_{2} \dot{f}_{5}-\bar{K}_{t} f_{5}^{\prime \prime}=0 \quad, \quad\left(\frac{L_{2}}{2}<x<\frac{7 L_{2}}{8}\right) \\
& \ddot{f}_{6}+\bar{C}_{2} \dot{f}_{6}-\bar{K}_{t} f_{6}^{\prime \prime}=0 \quad, \quad\left(\frac{7 L_{2}}{8}<x<L_{2}\right)
\end{aligned}
$$




$$
\begin{array}{ll}
\ddot{V}_{7}+\bar{K}_{3} V_{7}^{\prime \prime \prime \prime}+\bar{C}_{3} \dot{V}_{7}=-\ddot{W}_{B}, \quad\left(0<x<L_{3}\right) \\
\ddot{V}_{8}+\bar{K}_{3} V_{8}^{\prime \prime \prime \prime}+\bar{C}_{3} \dot{V}_{8}=-\ddot{W}_{B}, \quad\left(0<x<L_{4}\right)
\end{array}
$$

Equations (72-79) show the total dynamic solution of the nonlinear buckled system. To solve the free vibration of the linearized buckled system, the nonlinear, damping, and external force terms should be dropped, so that the equations of motion for different parts would be formed as follows:

$$
\begin{gathered}
\ddot{V}_{1}+\bar{K}_{1} V_{1}^{\prime \prime \prime \prime}+\left[\bar{P}-\bar{K}_{2} \int_{0}^{\frac{L_{1}}{2}} W_{1}^{s^{\prime 2}} d x\right] V_{1}^{\prime \prime}-2 \bar{K}_{2} W_{1}^{s^{\prime \prime}} \int_{0}^{\frac{L_{1}}{2}} W_{1}^{s^{\prime}} V_{1}^{\prime} d x=0 \\
\ddot{V}_{2}+\bar{K}_{1} V_{2}^{\prime \prime \prime \prime}+\left[\bar{P}-\bar{K}_{2} \int_{\frac{L_{1}}{2}}^{L_{1}}{W_{2}^{s^{\prime}}}^{2} d x\right] V_{2}^{\prime \prime}-2 \bar{K}_{2} W_{2}^{s^{\prime \prime}} \int_{\frac{L_{1}}{2}}^{L_{1}} W_{2}^{s^{\prime}} V_{2}^{\prime} d x=0 \\
\ddot{f}_{3}-\bar{K}_{t} f_{3}^{\prime \prime}=0 \\
\ddot{f}_{4}-\bar{K}_{t} f_{4}^{\prime \prime}=0 \\
\ddot{f}_{5}-\bar{K}_{t} f_{5}^{\prime \prime}=0 \\
\ddot{f}_{6}-\bar{K}_{t} f_{6}^{\prime \prime}=0 \\
\ddot{V}_{7}+\bar{K}_{3} V_{7}^{\prime \prime \prime}=0 \\
\ddot{V}_{8}+\bar{K}_{3} V_{8}^{\prime \prime \prime \prime}=0
\end{gathered}
$$

The following presumed solution including the time-dependent harmonic functions are used to get the dynamic natural frequencies and mode shapes of the linearized buckled system: 


$$
\begin{aligned}
& V_{i}=\psi_{i}(x) e^{j \omega t}, \quad(i=1,2,7,8) \\
& f_{i}=\psi_{i}(x) e^{j \omega t}, \quad(i=3,4,5,6)
\end{aligned}
$$

By inserting the above presumed solutions into the linearized equations of motion, the following relations could be derived for each part of the system:

$$
\begin{gathered}
\bar{K}_{1} \psi_{1}^{\prime \prime \prime \prime}+\left[\bar{P}-\bar{K}_{2} \int_{0}^{\frac{L_{1}}{2}}{W_{1}^{s^{\prime}}}^{2} d x\right] \psi_{1}^{\prime \prime}-2 \bar{K}_{2} W_{1}^{s^{\prime \prime}} \int_{0}^{\frac{L_{1}}{2}} W_{1}^{s^{\prime}} \psi_{1}^{\prime} d x-\omega^{2} \psi_{1}=0 \\
\bar{K}_{1} \psi_{2}^{\prime \prime \prime \prime}+\left[\bar{P}-\bar{K}_{2} \int_{\frac{L_{1}}{2}}^{L_{1}}{W_{2}^{s^{\prime}}}^{2} d x\right] \psi_{2}^{\prime \prime}-2 \bar{K}_{2} W_{2}^{s^{\prime \prime}} \int_{\frac{L_{1}}{2}}^{L_{1}} W_{2}^{s^{\prime}} \psi_{2}^{\prime} d x-\omega^{2} \psi_{2}=0 \\
\left\{\begin{array}{c}
\psi_{i}^{\prime \prime}+\beta_{3}^{2} \psi_{i}=0 \\
\beta_{3}=\left(\frac{1}{\bar{K}_{t}}\right) \omega^{2} \quad(i=3,4,5,6) \\
\left\{\begin{array}{c}
\psi_{i}^{\prime \prime \prime \prime}-\beta_{4}^{4} \psi_{i}=0 \\
\beta_{4}=\left(\frac{1}{\bar{K}_{3}}\right) \omega^{2}
\end{array}\right.
\end{array}\right.
\end{gathered}
$$

The general solutions for parts 3-8 are straightforward and would be as follows:

$$
\begin{array}{cc}
\psi_{3}(x)=A_{3} \sin \left(\beta_{3} x\right)+B_{3} \cos \left(\beta_{3} x\right), & 0 \leq x \leq \frac{L_{2}}{8} \\
\psi_{4}(x)=A_{4} \sin \left(\beta_{3} x\right)+B_{4} \cos \left(\beta_{3} x\right), & \frac{L_{2}}{8} \leq x \leq \frac{L_{2}}{2}
\end{array}
$$




$$
\begin{aligned}
& \psi_{5}(x)=A_{5} \sin \left(\beta_{3} x\right)+B_{5} \cos \left(\beta_{3} x\right), \quad \frac{L_{2}}{2} \leq x \leq \frac{7 L_{2}}{8} \\
& \psi_{6}(x)=A_{6} \sin \left(\beta_{3} x\right)+B_{6} \cos \left(\beta_{3} x\right), \quad \frac{7 L_{2}}{8} \leq x \leq L_{2} \\
& \psi_{7}(x)=A_{7} \sin \left(\beta_{4} x\right)+B_{7} \cos \left(\beta_{4} x\right)+C_{7} \sinh \left(\beta_{4} x\right)+D_{7} \cosh \left(\beta_{4} x\right), \\
& 0 \leq x \leq L_{3} \\
& \psi_{8}(x)=A_{8} \sin \left(\beta_{4} x\right)+B_{8} \cos \left(\beta_{4} x\right)+C_{8} \sinh \left(\beta_{4} x\right)+D_{8} \cosh \left(\beta_{4} x\right), \\
& 0 \leq x \leq L_{4}
\end{aligned}
$$

By applying the end conditions of the torsional rod and cantilever arms, eight unknowns of the above solutions can be eliminated and written in terms of others. These end conditions for the dynamic system would be as follows:

$$
\begin{aligned}
& \phi_{3}(0)=0, \quad \stackrel{\text { yields }}{\longrightarrow} \quad \psi_{3}(x)=A_{3} \sin \left(\beta_{3} x\right) \\
& \phi_{6}(0)=0, \quad \stackrel{\text { yields }}{\longrightarrow}\left\{\begin{array}{c}
\psi_{6}(x)=A_{6}\left[\sin \left(\beta_{3} x\right)-R_{6} \cos \left(\beta_{3} x\right)\right] \\
R_{6}=\frac{\sin \left(\beta_{3} L_{2}\right)}{\cos \left(\beta_{3} L_{2}\right)}
\end{array}\right.
\end{aligned}
$$

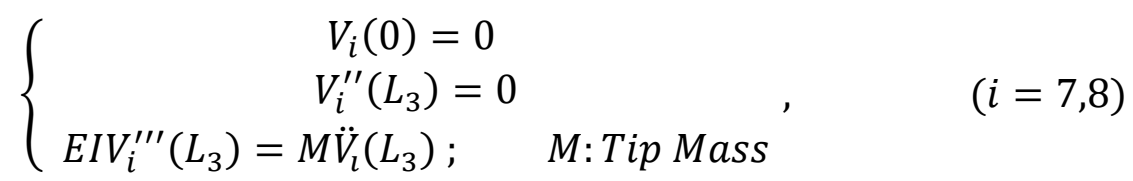

$$
\begin{aligned}
& \stackrel{\text { yields }}{\longrightarrow} \quad \psi_{i}(x)=A_{i}\left\{\left[\sin \left(\beta_{4} x\right)+R_{7 A}\left(\cos \left(\beta_{4} x\right)-\cosh \left(\beta_{4} x\right)\right)\right]-\right. \\
& \left.\frac{R_{7}}{R_{8}}\left[\sinh \left(\beta_{4} x\right)+R_{7 B}\left(\cos \left(\beta_{4} x\right)-\cosh \left(\beta_{4} x\right)\right)\right]\right\},(=7,8)
\end{aligned}
$$


Where

$$
\begin{gathered}
R_{7 A}=-\frac{\sin \left(\beta_{4} L_{3}\right)}{\cos \left(\beta_{4} L_{3}\right)+\cosh \left(\beta_{4} L_{3}\right)} ; \quad R_{7 B}=\frac{\sinh \left(\beta_{4} L_{3}\right)}{\cos \left(\beta_{4} L_{3}\right)+\cosh \left(\beta_{3} L_{3}\right)} \\
R_{7}=\left\{-\cos \left(\beta_{4} L_{3}\right)+R_{7 A}\left[\sin \left(\beta_{4} L_{3}\right)-\sinh \left(\beta_{4} L_{3}\right)\right]+\frac{M}{\bar{m}_{3}} \beta_{4}\left[\sin \left(\beta_{4} L_{3}\right)\right.\right. \\
\left.+R_{7 B}\left[\cos \left(\beta_{4} L_{3}\right)-\cosh \left(\beta_{4} L_{3}\right)\right]\right\} \\
R_{8}=\left\{\cosh \left(\beta_{4} L_{3}\right)+R_{7 A}\left[\sin \left(\beta_{4} L_{3}\right)-\sinh \left(\beta_{4} L_{3}\right)\right]+\frac{M}{\bar{m}_{3}} \beta_{4}\left[\sinh \left(\beta_{4} L_{3}\right)\right.\right. \\
\left.+R_{7 B}\left[\cos \left(\beta_{4} L_{3}\right)-\cosh \left(\beta_{4} L_{3}\right)\right]\right\}
\end{gathered}
$$

For finding the general solutions for parts 1 and 2, equations (90) and (91) are rewritten in the following form:

$$
\begin{aligned}
& \bar{K}_{1} \psi_{1}^{\prime \prime \prime \prime}+\bar{Z}_{1} \psi_{1}^{\prime \prime}-\omega^{2} \psi_{1}=2 \bar{K}_{2} W_{1}^{s^{\prime \prime}} \bar{\gamma}_{1} \\
& \bar{K}_{1} \psi_{2}^{\prime \prime \prime \prime}+\bar{Z}_{2} \psi_{2}^{\prime \prime}-\omega^{2} \psi_{2}=2 \bar{K}_{2} W_{2}^{s^{\prime \prime}} \bar{\gamma}_{2}
\end{aligned}
$$

where

$$
\begin{cases}\bar{\gamma}_{1}=\int_{0}^{\frac{L_{1}}{2}} W_{1}^{S^{\prime}} \psi_{1}^{\prime} d x ; & \bar{Z}_{1}=\left[\bar{P}-\bar{K}_{2} \int_{0}^{\frac{L_{1}}{2}}{W_{1}^{s^{\prime}}}^{2} d x\right] \\ \bar{\gamma}_{2}=\int_{\frac{L_{1}}{2}}^{L_{1}} W_{2}^{s^{\prime}} \psi_{2}^{\prime} d x ; & \bar{Z}_{2}=\left[\bar{P}-\bar{K}_{2} \int_{\frac{L_{1}}{2}}^{L_{1}} W_{2}^{s^{\prime 2}} d x\right]\end{cases}
$$

To solve the equations (104) and (105), the general solutions can be divided into homogenous and non-homogenous parts: 


$$
\begin{aligned}
& \psi_{1}=\psi_{1 h}+\psi_{1 p} \\
& \psi_{2}=\psi_{2 h}+\psi_{2 p}
\end{aligned}
$$

\section{I- Homogenous part:}

The homogenous part of the solutions is when the right-hand side of the equations (104) and (105) are zero:

$$
\begin{aligned}
& \bar{K}_{1} \psi_{1 h}^{\prime \prime \prime}+\bar{Z}_{1} \psi_{1 h}^{\prime \prime}-\omega^{2} \psi_{1 h}=0 \\
& \bar{K}_{1} \psi_{2 h}^{\prime \prime \prime \prime}+\bar{Z}_{2} \psi_{2 h}^{\prime \prime}-\omega^{2} \psi_{2 h}=0
\end{aligned}
$$

Due to the symmetry in the static shape of the central buckled beam, it can be shown that $\bar{Z}_{1}=\bar{Z}_{2}=\bar{Z}$, so the characteristic equations and general solutions for the homogenous parts of the central buckled beam would be as follows:

$$
\begin{aligned}
& \stackrel{\text { Chareacteristic Equation }}{\longrightarrow}\left\{\begin{array}{c}
D_{1}=\frac{\bar{Z}}{\bar{K}_{1}} \\
S_{2}=\frac{1}{\bar{K}_{1}} \omega^{2}
\end{array}\right. \\
& \stackrel{\text { eigenvalues }}{\longrightarrow}\left\{\begin{array}{c}
S_{1} D^{2}-S_{2}=0 \\
\beta_{1}=\left(-\frac{S_{1}}{2}+\frac{\sqrt{S_{1}^{2}+4 S_{2}}}{2}\right)^{\frac{1}{2}} \\
\beta_{2}=\left(+\frac{S_{1}}{2}+\frac{\sqrt{S_{1}^{2}+4 S_{2}}}{2}\right)^{\frac{1}{2}}
\end{array}\right. \\
& \psi_{1}(x)=A_{1} \sin \left(\beta_{1} x\right)+B_{1} \cos \left(\beta_{1} x\right)+C_{1} \sinh \left(\beta_{2} x\right)+D_{1} \cosh \left(\beta_{2} x\right),
\end{aligned}
$$




$$
\begin{gathered}
\psi_{2}(x)=A_{2} \sin \left(\beta_{1} x\right)+B_{2} \cos \left(\beta_{1} x\right)+C_{2} \sinh \left(\beta_{2} x\right)+D_{2} \cosh \left(\beta_{2} x\right), \\
\frac{L_{1}}{2} \leq x \leq L_{1}
\end{gathered}
$$

II- Non-homogenous part:

The particular solutions of the equations (104) and (105) can be formed as follows:

$$
\begin{aligned}
& \psi_{1 p}(x)=E_{1} \cos \left(\beta_{s} x\right)+F_{1} \sin \left(\beta_{s} x\right) \\
& \psi_{2 p}(x)=E_{2} \cos \left(\beta_{s} x\right)+F_{2} \sin \left(\beta_{s} x\right)
\end{aligned}
$$

By putting these particular solutions into the equations (104) and (105) followed by some mathematical simplifications, the coefficients of the particular solutions can be written as functions of the homogenous unknown constants:

$$
\left\{\begin{array}{l}
E_{i}=\frac{-2 \bar{K}_{2} G_{i}\left[\gamma_{A i} A_{i}+\gamma_{B i} B_{i}+\gamma_{C i} C_{i}+\gamma_{D i} D_{i}\right]}{-\beta_{S}^{4} \bar{K}_{1}+\beta_{S}^{2} \bar{Z}+\omega^{2}+2 \bar{K}_{2}\left[\gamma_{G i} G_{i}+\gamma_{F i} F_{i}\right]} \\
F_{i}=\frac{-2 \bar{K}_{2} H_{i}\left[\gamma_{A i} A_{i}+\gamma_{B i} B_{i}+\gamma_{C i} C_{i}+\gamma_{D i} D_{i}\right]}{-\beta_{S}^{4} \bar{K}_{1}+\beta_{S}^{2} \bar{Z}+\omega^{2}+2 \bar{K}_{2}\left[\gamma_{G i} G_{i}+\gamma_{F i} F_{i}\right]}
\end{array}, \quad(i=1,2)\right.
$$

where $\gamma_{A}, \gamma_{B}, \gamma_{C}, \gamma_{D}, \gamma_{G}, \gamma_{F}, G_{1}, G_{2}, F_{1}, F_{2}$ are known constants. Finally, the total general solutions of the linearized equations for parts 1 and 2 can be written only in terms of the unknown coefficients of the homogenous part. Next, applying the remaining end conditions for the main beam (which gives 6 relations for parts 1 and 2) leads to the general solutions of the linearized buckled beam in a format that includes only 1 unknown constant for each part:

$$
\begin{gathered}
\psi_{i}(x)=A_{i}\left\{R_{A i} \sin \left(\beta_{1} x\right)+R_{B i} \cos \left(\beta_{1} x\right)+R_{C i} \sinh \left(\beta_{2} x\right)+R_{D i} \cosh \left(\beta_{2} x\right)\right. \\
\left.+R_{E i} \cos \left(\beta_{S} x\right)+R_{F i} \sin \left(\beta_{S} x\right)\right\} ; \quad(i=1,2)
\end{gathered}
$$


Parameters $R_{A i}, R_{B i}, R_{C i}, R_{D i}, R_{E i}, R_{F i}$ are known constants obtained from applying the end boundary conditions of the main beam.

So far, the general solutions for each part of the system are obtained in the described forms after applying all end conditions, which reduce to 14 unknown constants in total. To find the remaining 10 unknown coefficients as well as the natural frequencies of the linearized system, these functions need to be solved in a coupled system by applying matching conditions. Applying these conditions (equations 63-68) results in a matrix-form equation with zero terms on the right-hand side:

$$
\left[B^{D}\right]\{A\}=\{0\}
$$

where the $10 \times 10$ matrix $\left[B^{D}\right]$ is the dynamic transformation matrix and vector $\{A\}$ is

$$
\{A\}=\left\{A_{1}, A_{2}, A_{3}, A_{4}, B_{4}, A_{5}, B_{5}, A_{6}, A_{7}, A_{8}\right\}^{T}
$$

The required conditions for non-zero solution of the equation (117) will give the characteristic equations from which one may obtain the system natural frequencies and mode shapes.

\subsubsection{Dynamics of the Nonlinear System}

In order to solve the nonlinear system of equations, a Galerkin approach was chosen to discretize the partial differential equations. This method requires spatial functions as the basis around which equations (72-79) can be discretized. It has been shown in the previous chapter that choosing functions which only satisfy the geometrical end boundaries produces results with neither the expected physical behavior, nor that observed in experimental testing. Using shape functions obtained from solving the free vibration case 
of the linearized buckled system, which satisfy both all the end and matching conditions, could resolve this issue.

In this section, Galerkin's method is used to discretize the nonlinear partial differential equations. It is assumed that

$$
\begin{array}{ll}
V_{j}(x, t)=\sum_{i=1}^{N} \psi_{j}^{i}(x) q_{j}^{i}(t), & (j=1,2,7,8) \\
f_{j}(x, t)=\sum_{i=1}^{N} \psi_{j}^{i}(x) q_{j}^{i}(t), & (j=3,4,5,6)
\end{array}
$$

where $N$ is the number of considered modes and $\psi^{i}(x)$ is the shape function of the system (relations 94-99 and 106 and 107) for $i^{\text {th }}$ mode. By putting these presumed solutions into the nonlinear equations of motion and applying Galerkin discretization process on each part domain, the discretized ordinary differential equation of motion for each part would be obtained as follows:

$$
\begin{gathered}
\sum_{j=1}^{N} m_{i j}^{1} \ddot{q}_{1}^{j}+\sum_{j=1}^{N} c_{i j}^{1} \dot{q}_{1}^{j}+\sum_{j=1}^{N} k_{i j}^{1} q_{1}^{j}+\sum_{j=1}^{N} \sum_{k=1}^{N} A_{i j k}^{1} q_{1}^{j} q_{1}^{k}+\sum_{j=1}^{N} \sum_{k=1}^{N} \sum_{l=1}^{N} B_{i j k l}^{1} q_{1}^{j} q_{1}^{k} q_{1}^{l} \\
+f_{1}^{i}=0 ;(i=1,2, \ldots, N) \\
\sum_{j=1}^{N} m_{i j}^{2} \ddot{q}_{2}^{j}+\sum_{j=1}^{N} c_{i j}^{2} \dot{q}_{2}^{j}+\sum_{j=1}^{N} k_{i j}^{2} q_{2}^{j}+\sum_{j=1}^{N} \sum_{k=1}^{N} A_{i j k}^{2} q_{2}^{j} q_{2}^{k}+\sum_{j=1}^{N} \sum_{k=1}^{N} \sum_{l=1}^{N} B_{i j k l}^{2} q_{2}^{j} q_{2}^{k} q_{2}^{l} \\
+f_{2}^{i}=0 ;(i=1,2, \ldots, N)
\end{gathered}
$$




$$
\begin{aligned}
& \sum_{j=1}^{N} m_{i j}^{3} \ddot{q}_{3}^{j}+\sum_{j=1}^{N} c_{i j}^{3} \dot{q}_{3}^{j}+\sum_{j=1}^{N} k_{i j}^{3} q_{3}^{j}=0 ; \quad(i=1,2, \ldots, N) \\
& \sum_{j=1}^{N} m_{i j}^{4} \ddot{q}_{4}^{j}+\sum_{j=1}^{N} c_{i j}^{4} \dot{q}_{4}^{j}+\sum_{j=1}^{N} k_{i j}^{4} q_{4}^{j}=0 ; \quad(i=1,2, \ldots, N) \\
& \sum_{j=1}^{N} m_{i j}^{5} \ddot{q}_{5}^{j}+\sum_{j=1}^{N} c_{i j}^{5} \dot{q}_{5}^{j}+\sum_{j=1}^{N} k_{i j}^{5} q_{5}^{j}=0 ; \quad(i=1,2, \ldots, N) \\
& \sum_{j=1}^{N} m_{i j}^{6} \ddot{q}_{6}^{j}+\sum_{j=1}^{N} c_{i j}^{6} \dot{q}_{6}^{j}+\sum_{j=1}^{N} k_{i j}^{6} q_{6}^{j}=0 ; \quad(i=1,2, \ldots, N) \\
& \sum_{j=1}^{N} m_{i j}^{7} \ddot{q}_{7}^{j}+\sum_{j=1}^{N} c_{i j}^{7} \dot{q}_{7}^{j}+\sum_{j=1}^{N} k_{i j}^{7} q_{7}^{j}+f_{7}^{i}=0 ; \quad(i=1,2, \ldots, N) \\
& \sum_{j=1}^{N} m_{i j}^{8} \ddot{q}_{8}^{j}+\sum_{j=1}^{N} c_{i j}^{8} \dot{q}_{8}^{j}+\sum_{j=1}^{N} k_{i j}^{8} q_{8}^{j}+f_{8}^{i}=0 ; \quad(i=1,2, \ldots, N)
\end{aligned}
$$

where the coefficients of each part are

Part 1:

$$
\begin{gathered}
m_{i j}^{1}=\int_{0}^{\frac{L_{1}}{2}} \psi_{1}^{i} \psi_{1}^{j} d x ; \quad c_{i j}^{1}=\bar{C}_{1} m_{i j}^{1} ; \quad f_{1}^{i}=\int_{0}^{\frac{L_{1}}{2}} \ddot{W}_{B} \psi_{1}^{i} d x \\
k_{i j}^{1}=\bar{K}_{1} \int_{0}^{\frac{L_{1}}{2}} \psi_{1}^{j^{\prime \prime \prime \prime}} \psi_{1}^{i} d x+\left[\bar{P}-\bar{K}_{2} \int_{0}^{\frac{L_{1}}{2}} W_{1}^{s^{\prime 2}} d x \int_{0}^{\frac{L_{1}}{2}} \psi_{1}^{j^{\prime \prime}} \psi_{1}^{i} d x\right. \\
-2 \bar{K}_{2} \int_{0}^{\frac{L_{1}}{2}} W_{1}^{s^{\prime \prime}} \psi_{1}^{i} d x \int_{0}^{\frac{L_{1}}{2}} W_{1}^{s^{\prime}} \psi_{1}^{j^{\prime}} d x
\end{gathered}
$$




$$
\begin{aligned}
& A_{i j k}^{1}=-\bar{K}_{2} \int_{0}^{\frac{L_{1}}{2}} W_{1}^{s^{\prime \prime}} \psi_{1}^{i} d x \int_{0}^{\frac{L_{1}}{2}} \psi_{1}^{j^{\prime}} \psi_{1}^{k^{\prime}} d x-2 \bar{K}_{2} \int_{0}^{\frac{L_{1}}{2}} \psi_{1}^{j^{\prime \prime}} \psi_{1}^{i} d x \int_{0}^{\frac{L_{1}}{2}} W_{1}^{s^{\prime}} \psi_{1}^{k^{\prime}} d x \\
& B_{i j k l}^{1}=-\bar{K}_{2} \int_{0}^{\frac{L_{1}}{2}} \psi_{1}^{j^{\prime \prime}} \psi_{1}^{i} d x \int_{0}^{2} \psi_{1}^{k^{\prime}} \psi_{1}^{l^{\prime}} d x
\end{aligned}
$$

Part 2:

$$
\begin{aligned}
& m_{i j}^{2}=\int_{\frac{L_{1}}{2}}^{L_{1}} \psi_{2}^{i} \psi_{2}^{j} d x ; \quad c_{i j}^{2}=\bar{C}_{1} m_{i j}^{2} ; \quad f_{2}^{i}=\int_{\frac{L_{1}}{2}}^{L_{1}} \ddot{W}_{B} \psi_{2}^{i} d x \\
& k_{i j}^{2}=\bar{K}_{1} \int_{\frac{L_{1}}{2}}^{L_{1}} \psi_{2}^{j^{\prime \prime \prime \prime}} \psi_{2}^{i} d x+\left[\bar{P}-\bar{K}_{2} \int_{\frac{L_{1}}{2}}^{L_{1}} W_{2}^{s^{\prime}}{ }^{2} d x\right] \int_{\frac{L_{1}}{2}}^{L_{1}} \psi_{2}^{j^{\prime \prime}} \psi_{2}^{i} d x \\
& -2 \bar{K}_{2} \int_{\frac{L_{1}}{2}}^{L_{1}} W_{2}^{s^{\prime \prime}} \psi_{2}^{i} d x \int_{\frac{L_{1}}{2}}^{L^{\prime}} W_{2}^{s^{\prime}} \psi_{2}^{j^{\prime}} d x \\
& A_{i j k}^{2}=-\bar{K}_{2} \int_{\frac{L_{1}}{2}}^{L_{1}} W_{2}^{s^{\prime \prime}} \psi_{2}^{i} d x \int_{\frac{L_{1}}{2}}^{L_{1}} \psi_{2}^{j^{\prime}} \psi_{2}^{k^{\prime}} d x-2 \bar{K}_{2} \int_{\frac{L_{1}}{2}}^{L_{1}} \psi_{2}^{j^{\prime \prime}} \psi_{2}^{i} d x \int_{\frac{L_{1}}{2}}^{L_{1}} W_{2}^{s^{\prime}} \psi_{2}^{k^{\prime}} d x \\
& B_{i j k l}^{2}=-\bar{K}_{2} \int_{\frac{L_{1}}{2}}^{L_{1}} \psi_{2}^{j^{\prime \prime}} \psi_{2}^{i} d x \int_{\frac{L_{1}}{2}}^{k_{1}} \psi_{2}^{k^{\prime}} \psi_{2}^{l^{\prime}} d x
\end{aligned}
$$

Part 3:

$$
m_{i j}^{3}=\int_{0}^{\frac{L_{2}}{8}} \psi_{3}^{i} \psi_{3}^{j} d x \quad ; \quad c_{i j}^{3}=\bar{C}_{2} m_{i j}^{3} \quad ; \quad k_{i j}^{3}=-\bar{K}_{t} \int_{0}^{\frac{L_{2}}{8}} \psi_{3}^{j^{\prime \prime}} \psi_{3}^{i} d x
$$

Part 4:

$$
m_{i j}^{4}=\int_{\frac{L_{2}}{8}}^{\frac{L_{2}}{2}} \psi_{4}^{i} \psi_{4}^{j} d x \quad ; \quad c_{i j}^{4}=\bar{C}_{2} m_{i j}^{4} \quad ; \quad k_{i j}^{4}=-\bar{K}_{t} \int_{\frac{L_{2}}{8}}^{\frac{L_{2}}{2}} \psi_{4}^{j^{\prime \prime}} \psi_{4}^{i} d x
$$

Part 5: 
$m_{i j}^{5}=\int_{\frac{L_{2}}{2}}^{\frac{7 L_{2}}{8}} \psi_{5}^{i} \psi_{5}^{j} d x \quad ; \quad c_{i j}^{5}=\bar{C}_{2} m_{i j}^{5} \quad ; \quad k_{i j}^{5}=-\bar{K}_{t} \int_{\frac{L_{2}}{2}}^{\frac{7 L_{2}}{8}} \psi_{5}^{j^{\prime \prime}} \psi_{5}^{i} d x$

Part 6:

$m_{i j}^{6}=\int_{\frac{7 L_{2}}{8}}^{L_{2}} \psi_{6}^{i} \psi_{6}^{j} d x \quad ; \quad c_{i j}^{6}=\bar{C}_{2} m_{i j}^{6} \quad ; \quad k_{i j}^{6}=-\bar{K}_{t} \int_{\frac{7 L_{2}}{8}}^{L_{2}} \psi_{6}^{j^{\prime \prime}} \psi_{6}^{i} d x$

Part 7:

$m_{i j}^{7}=\int_{0}^{L_{3}} \psi_{7}^{i} \psi_{7}^{j} d x ; \quad c_{i j}^{7}=\bar{C}_{3} m_{i j}^{7} ; \quad k_{i j}^{7}=\bar{K}_{3} \int_{0}^{L_{3}} \psi_{7}^{j^{\prime \prime \prime \prime}} \psi_{7}^{i} d x ; \quad f_{7}^{i}=\int_{0}^{L_{3}} \ddot{W}_{B} \psi_{7}^{i} d x$

Part 8:

$m_{i j}^{8}=\int_{0}^{4} \psi_{8}^{i} \psi_{8}^{j} d x \quad ; \quad c_{i j}^{8}=\bar{C}_{4} m_{i j}^{8} \quad ; \quad k_{i j}^{8}=\bar{K}_{3} \int_{0}^{L_{4}} \psi_{8}^{j^{\prime \prime \prime \prime}} \psi_{8}^{i} d x \quad ; \quad f_{8}^{i}=\int_{0}^{L_{4}} \ddot{W}_{B} \psi_{8}^{i} d x$

To solve these discretized equations in one system, first, the generalized coordinates of each part needs to be combined to one coordinate vector. If $N$ modes are considered for each part, the total generalized coordinate vector of the whole system can be written as follows:

$$
\begin{gathered}
\underline{q}_{8 N \times 1}=\left\{\begin{array}{c}
\left\{q_{1}^{1}, q_{1}^{2}, \ldots, q_{1}^{N}\right\}^{T},\left\{q_{2}^{1}, q_{2}^{2}, \ldots, q_{2}^{N}\right\}^{T},\left\{q_{3}^{1}, q_{3}^{2}, \ldots, q_{3}^{N}\right\}^{T},\left\{q_{4}^{1}, q_{4}^{2}, \ldots, q_{4}^{N}\right\}^{T}, \\
\left\{q_{5}^{1}, q_{5}^{2}, \ldots, q_{5}^{N}\right\}^{T},\left\{q_{6}^{1}, q_{6}^{2}, \ldots, q_{6}^{N}\right\}^{T},\left\{q_{7}^{1}, q_{7}^{2}, \ldots, q_{7}^{N}\right\}^{T},\left\{q_{8}^{1}, q_{8}^{2}, \ldots, q_{8}^{N}\right\}^{T}
\end{array}\right\} \\
=\left\{\underline{q}_{N \times 1}, \underline{q}_{2 \times 1}, \underline{q}_{3 \times 1}, \underline{q}_{4 \times 1}, \underline{q}_{5 \times 1}, \underline{q}_{6 \times 1}, \underline{q}_{N \times 1}, \underline{q}_{8 \times 1}\right\}^{T}
\end{gathered}
$$

As can be seen from the above relation, the total degrees of freedom for the uncoupled system is $8 N$. By writing equations (51-53) in a matrix form and replacing the total generalized coordinates vector formed in equation (54), the uncoupled discretized nonlinear equations of the whole system can be written as: 


$$
[M] \underline{\ddot{q}}+[C] \underline{\dot{q}}+[K] \underline{q}+\underline{N V}=\underline{F}
$$

where $8 \times 8$ matrices $[M],[C],[K]$ are the system mass, damping and stiffness matrix, $\underline{N V}$ is the nonlinear terms vector, which includes both quadratic and cubic terms, and $F$ is the system external force vector.

As mentioned above, the obtained system of equations is for the uncoupled structure. These time-dependent generalized coordinates need to be solved subject to the coupling conditions. Thus, the next step is linking these coordinates together. Considering the fact that the boundary conditions of the linear and nonlinear systems are the same, the matching conditions to be applied on the nonlinear system to couple different parts together are the same as was done for the linear case. For each mode considered, it is possible to pick one generalized coordinate as independent and write the other seven coordinates in terms of the independent one through the matching conditions. These relations can be written as a coordinate transformation. Since analyzing the motion of the main buckled beam in the system is the ultimate goal, the generalized coordinate of part 1 is chosen as the independent coordinate and for mode $i$ and the following transformation relation can be formed using geometrical matching conditions (equations $63,65,67$ ):

$$
\underline{q}^{i}=\left[B^{i}\right] q_{1}^{i} \quad ; \quad(i=1,2, \ldots, N)
$$

where $\left[B^{i}\right]$ is the $8 \times 1$ coordinate transformation matrix for the $i^{\text {th }}$ mode. It noted that force-moment matching conditions will be automatically satisfied via using the true shape functions of the linearized system. By doing this for all $N$ modes considered, a relation between the generalized coordinates of the $1^{\text {st }}$ part and total generalized coordinate system can be built as follows: 


$$
\underline{q}=[B] \underline{q_{1}}
$$

$[B]$ is an $8 N \times N$ coupling transformation matrix that relates the total generalized coordinates to the generalized coordinate of part 1. Solving the system equation (130) subject to the transformation relation (132) requires the use of Lagrange multipliers to constrain the system [80], which results in the following equation:

$$
\left[M_{u}\right] \underline{\ddot{q}_{1}}+\left[C_{u}\right] \underline{\dot{q}_{1}}+\left[K_{u}\right] \underline{q_{1}}+\underline{N V_{u}}=\underline{F_{u}}
$$

where

$$
\begin{gathered}
{\left[M_{u}\right]=[B]^{T}[M][B], \quad\left[C_{u}\right]=[B]^{T}[C][B], \quad\left[K_{u}\right]=[B]^{T}[K][B],} \\
\underline{N V_{u}}=[B]^{T} \underline{N V}, \quad \underline{F_{u}}=[B]^{T} \underline{F}
\end{gathered}
$$

Equation (133) can be recast in the first-order form and solved numerically using the Runge-Kutta algorithm, which produces the generalized coordinates of part 1 . Consequently, the total nonlinear solution for part 1 can be written in the following form:

$$
W_{1}(x, t)=W_{1}^{s}(x)+\sum_{i=1}^{N} \psi_{1}^{i}(x) q_{1}^{i}(t)
$$

The form of the general solution (134) can be written for all other parts of the system by using their own static and dynamic shape functions and transformation relation (132).

\subsection{Model Validation}

To validate the model proposed in this chapter, the experimental setup shown in Figure 4 has been considered again. A finite element model of the described device was also developed in ANSYS 18.1. BEAM 188 was selected as the element type and the number of nodes considered for the central buckled beam, torsional rod, and cantilever arms was 60,40 , and 30 respectively. Table 2 shows the critical buckling load and the first three 
natural frequencies of the tested device developed by the proposed model and finite element analysis. These natural frequencies are developed for a slightly buckled system; i.e. compressive load is $P_{c r}=34 \mathrm{~N}$, which is just above the critical buckling load. The first natural frequency of the printed device was also experimentally measured and listed in this table (the higher frequencies are beyond the limit of the shaker table). Note that the first natural frequency of the system does not change substantially as the buckling load is increased, since this response is dominated by the behavior of the cantilever arms, which are the most compliant part of the structure. As pointed out previously, his feature is designed to increase the system flexibility at lower excitation frequencies, aiding its functionality as a vibration energy harvester. As can be seen from the table, the error between the model and FEA results is less than $1 \%$ for all comparisons, which shows a very good agreement between the developed model and finite element analysis. Moreover, the measured first natural frequency of the experimental setup is in good agreement with the modeling results, which verifies the proposed model in this study.

Table 2. Critical buckling load and natural frequencies of the experimentally tested device (frequencies of higher modes are beyond the limit of the shaker table).

\begin{tabular}{|c|c|c|c|c|}
\hline & $\begin{array}{c}\text { Critical Buckling } \\
\text { Load }(N)\end{array}$ & $1^{\text {st }}$ Mode $(\mathrm{Hz})$ & $2^{\text {nd }}$ Mode $(\mathrm{Hz})$ & $3^{\text {rd }}$ Mode $(\mathrm{Hz})$ \\
\hline Model & 33.95 & 23.54 & 433.97 & 1426.65 \\
\hline $\begin{array}{c}\text { FEA } \\
(\% \text { deviation })\end{array}$ & $33.89(0.18 \%)$ & $23.45(0.38 \%)$ & $431.83(0.49 \%)$ & $1417.26(0.66 \%)$ \\
\hline $\begin{array}{c}\text { Experiment } \\
(\% \text { deviation })\end{array}$ & - & $24.90(5.77 \%)$ & - & - \\
\hline
\end{tabular}


To verify the bistability of the studied structure in the model, the buckled shape of the main beam is plotted for the $40 \mathrm{~N}$ buckling load, which is above the critical buckling load listed in Table 2 (Figure 10). Two stable states corresponding to the buckled configurations of the central beam and zero unstable state, which plays as a transition between two stable states, are described in this figure. It proves that for compressive loads above the critical buckling one, bistability occurs in the proposed model (Note that any load higher than the critical buckling load of the system (33.95 N) makes the system bistable).

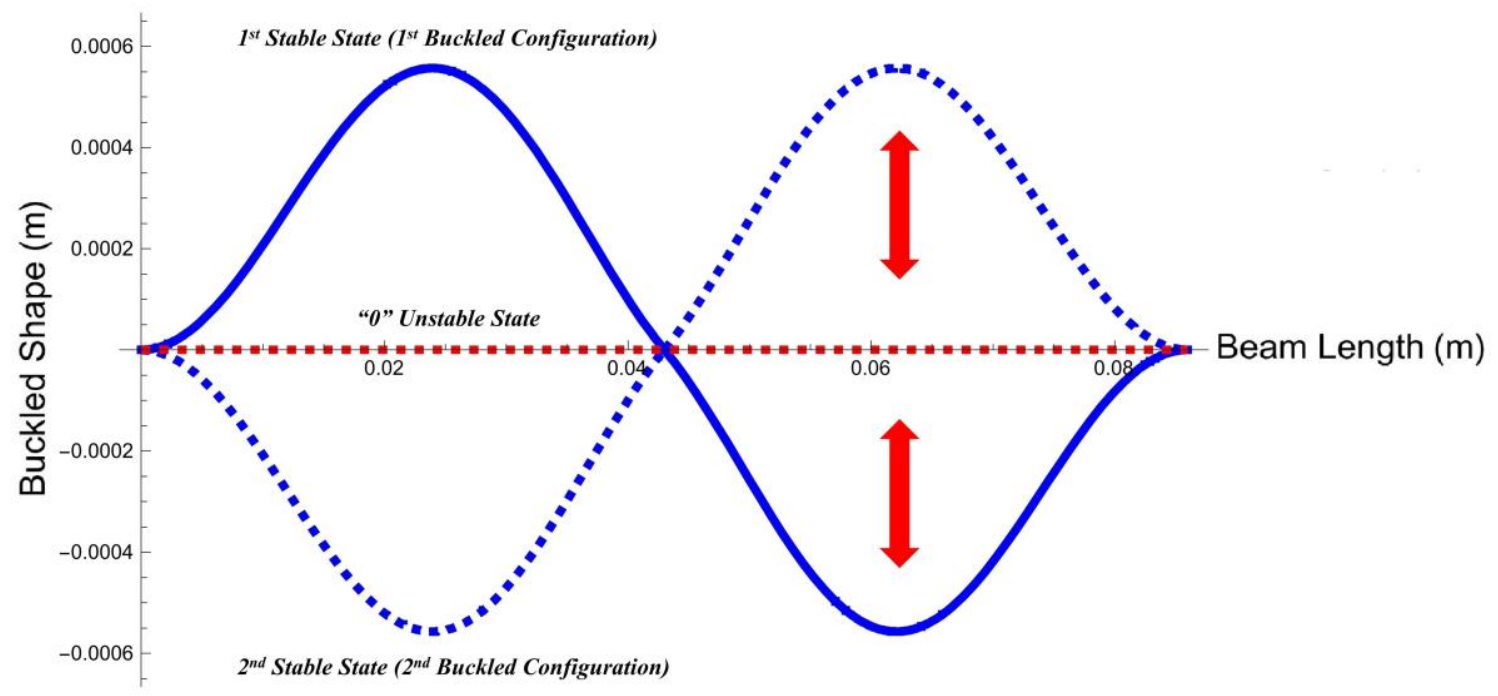

Figure 10. Three post buckling states (two buckled stable states and one zero unstable state) of the central beam for the experimental device under compressive load $P=40 \mathrm{~N}$.

Another case of the amplitude-frequency response of the printed device is shown for both modeling and experimental results in Figure 11. The compressive load and base excitation amplitude are $35 \mathrm{~N}$ and $2 \mathrm{~mm}$ respectively. This figure shows qualitatively good agreement between the developed model and experimental results. It needs to be pointed out that there are various factors affecting the existing discrepancy between the model and 
experiment, including measuring uncertainties, experimental setup and 3D printing imperfections, simplifications considered in the model etc. Regime behavior modeled is consistent with the previous experimental works [79, 82].

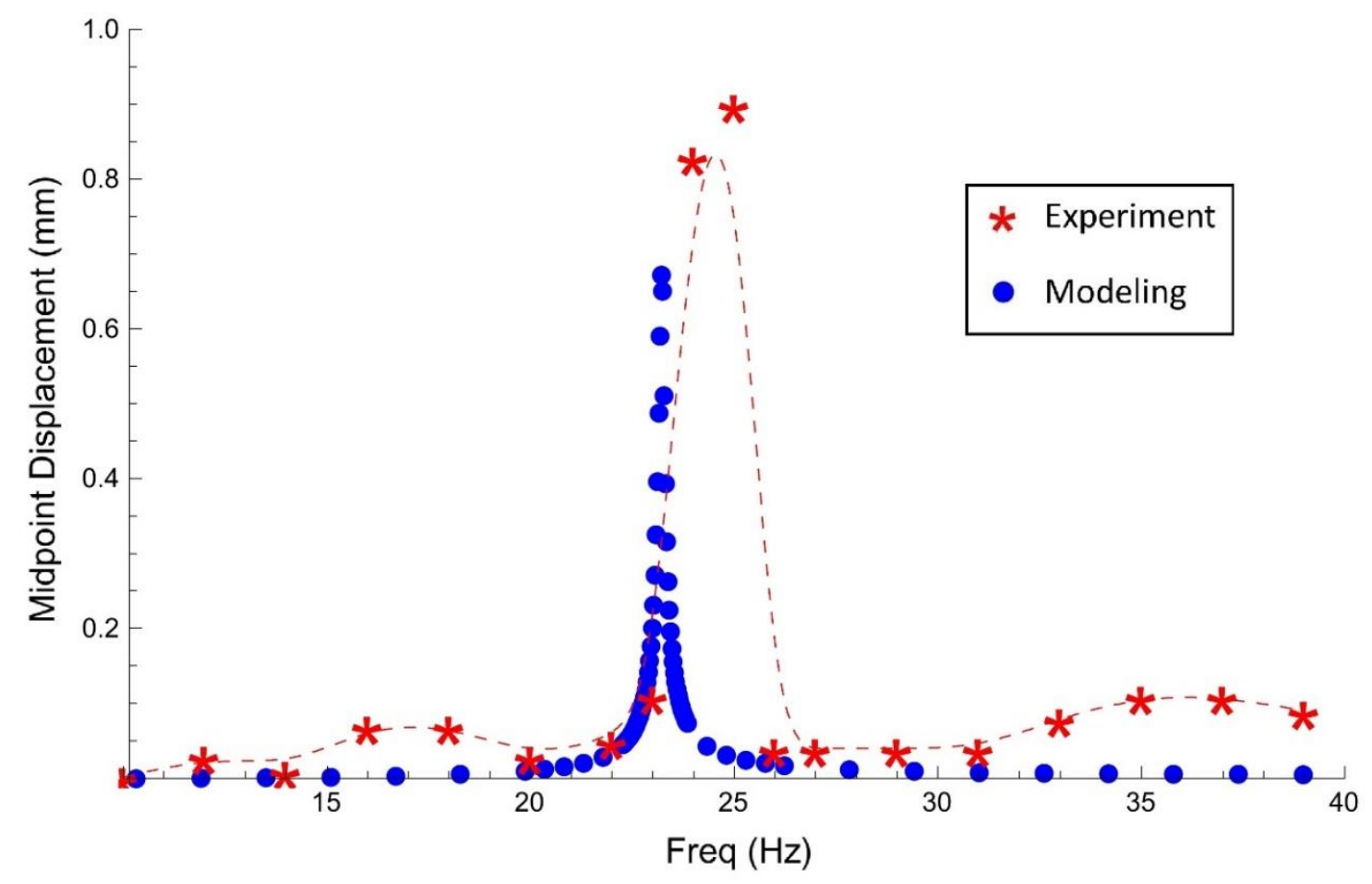

Figure 11. Amplitude-frequency response of the central buckled beam of the experimental device with $35 \mathrm{~N}$ buckling load and $2 \mathrm{~mm}$ base excitation.

\subsection{Results and Discussion}

After validating the proposed model by comparison with both finite element analysis and experimental data, amplitude-frequency response and snap-through regimes are developed based on the modeling approach to show the device performance as a vibration energy harvester. All the results showing in this section are developed for the experimental device described in Figure 4. Figures 12-14 show the modeling amplitude-frequency response of the post-buckled device around the first mode of vibration with four different base 
excitations for three different buckling loads applied on the central beam. The location of the measurement for these results corresponds to the vertical motion of the midpoint of half of the main buckled beam (midpoint of part 1). The hardening behavior of the system is obvious for the post-buckled configuration at its first mode and the hysteresis behavior is more pronounced as the base excitation (listed in terms of applied displacement amplitude) is increased for all three buckling load cases. Moreover, it can be observed from Figures 12-14 that by increasing the buckling load from $35 N$ (Figure 12), which is just beyond the first critical load and indicates a moderately post-buckled situation, to the higher values (Figures 13 and 14), the system becomes stiffer. Consequently, the frequency at which maximum amplitude of the main beam occurs shifts toward higher exciting frequencies with increase in the applied buckling load. As reported previously with this structure [79], the experimental frequency sweep direction (increasing or decreasing) could influence the observed hysteresis behavior of the structure amplitude-frequency response.

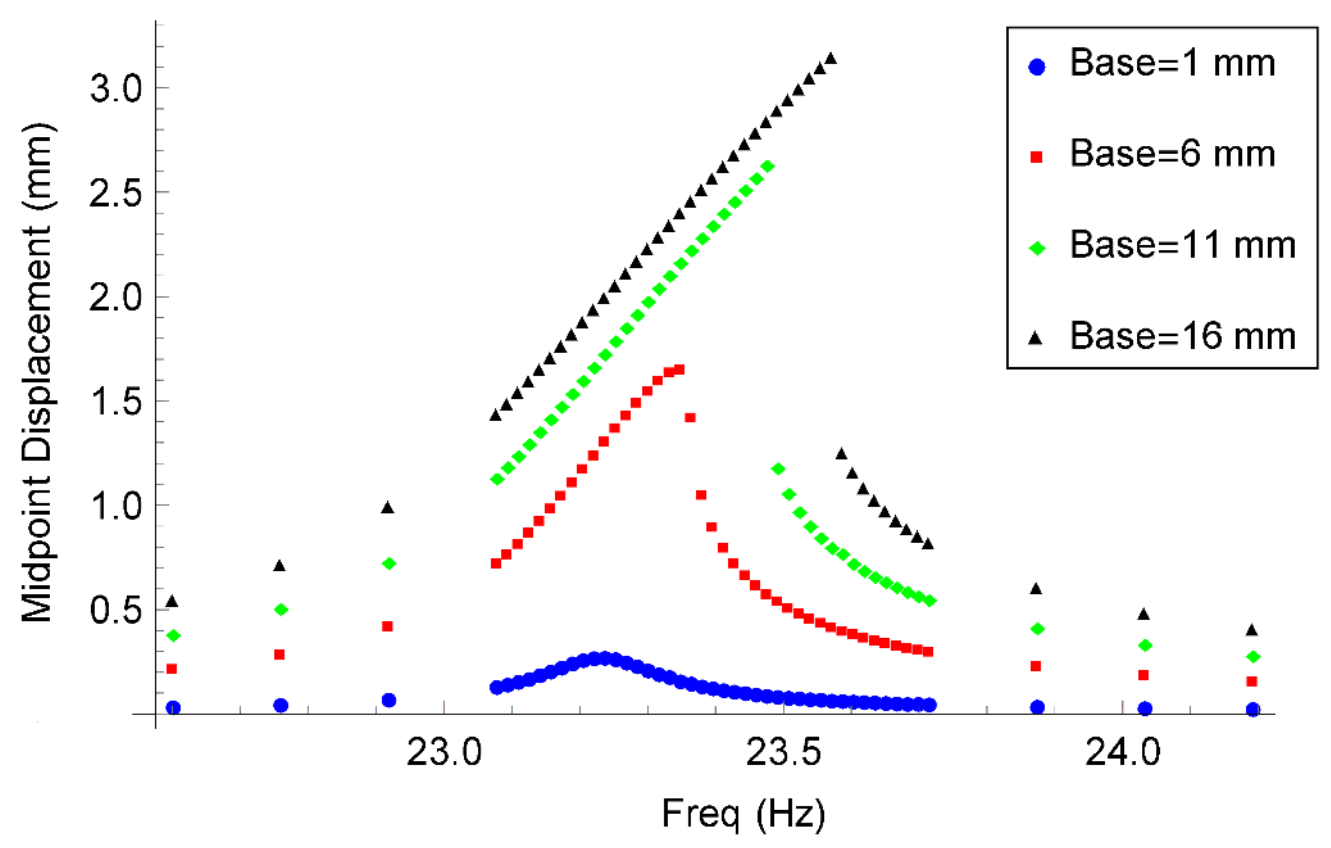


Figure 12. Amplitude-frequency response of the central buckled beam of the experimental device with four different base excitations for the buckling load $P=35 \mathrm{~N}$.

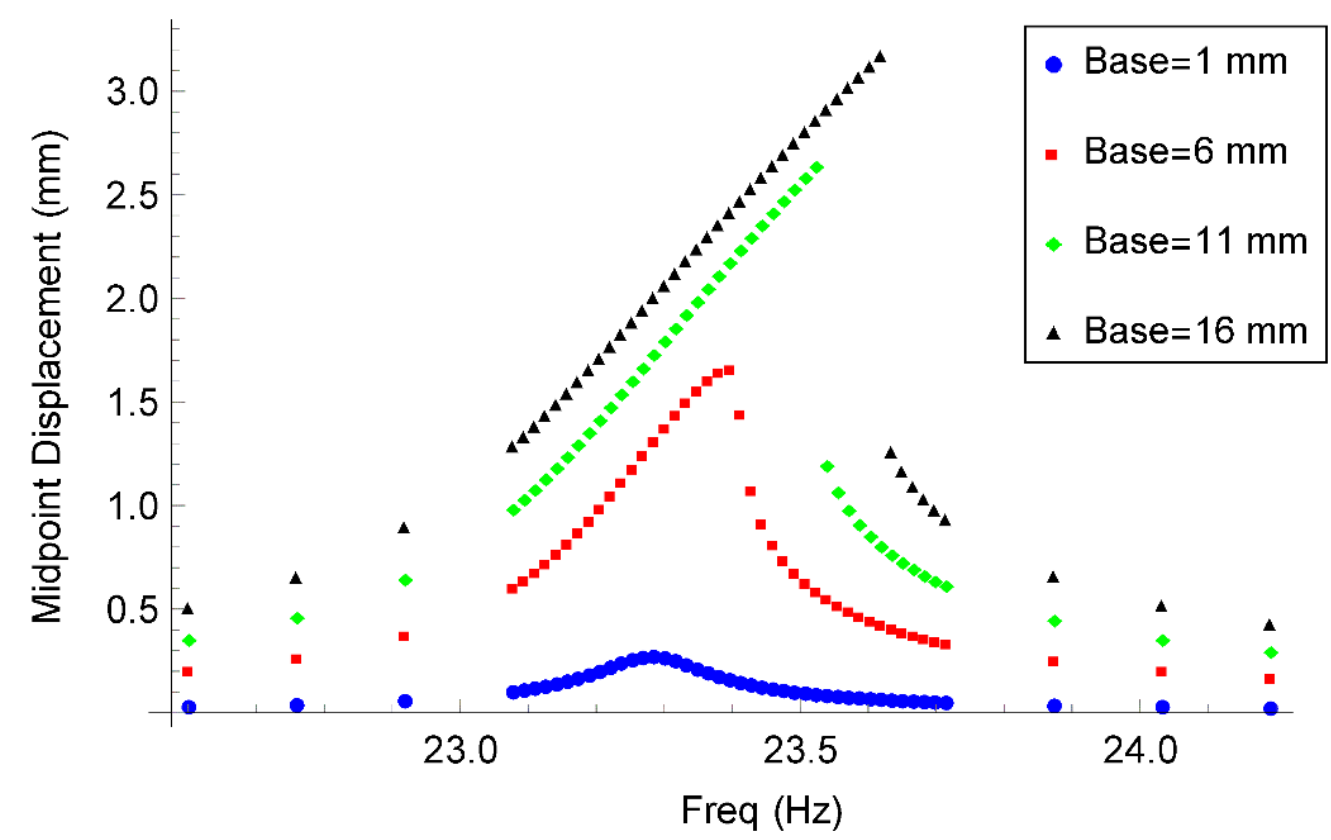

Figure 13. Amplitude-frequency response of the central buckled beam of the experimental device with four different base excitations for the buckling load $P=45 \mathrm{~N}$. 


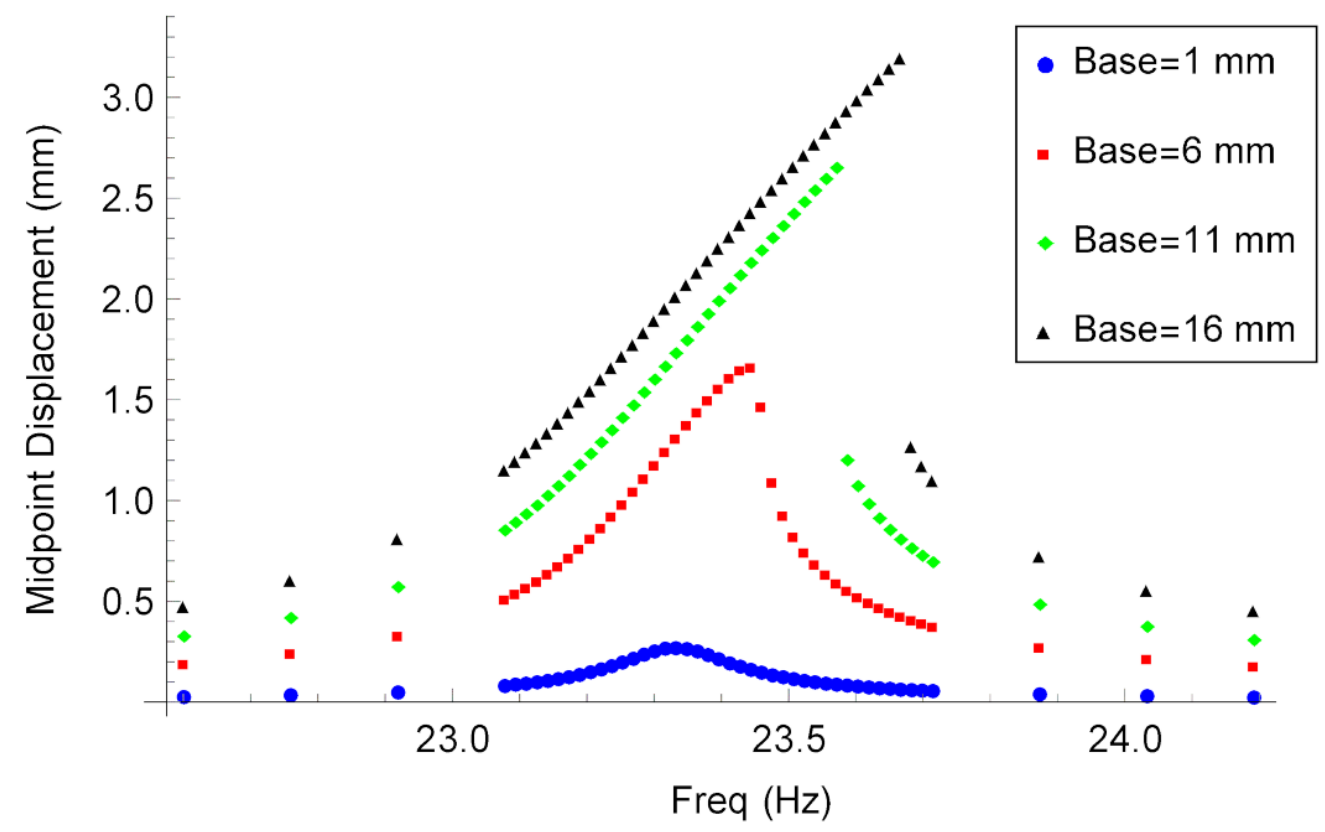

Figure 14. Amplitude-frequency response of the central buckled beam of the experimental device with four different base excitations for the buckling load $P=55 \mathrm{~N}$.

As discussed in section 4.3.1, the post-buckled system has three potential responses for the static case: two stable buckled states and one unstable flat compressed state. Since in the nonlinear modeling consideration, the dynamic response of each part is considered around one of the system stable solutions, capturing the exact snap-through motion (in which the system moves between its two stable states) is not possible for such coupled structure. However, the threshold of snap-through can be developed by measuring the amplitude required in the potential energy diagram for the stable solution to reach the zero unstable solution. This would indicate the minimum required base amplitude to instigate snapthrough.

Figures 15-17 show the model results for regimes of snap-through that corresponds to the experimental setup for three different buckling loads. For each part of the system, three 
modes are considered, leading to a total of 24 generalized coordinates. In all plots, "ST" stands for the snap-through (switching between stable buckled states) regimes and "NST" designates the regimes with no snap-through behavior. As can be seen from Figures 15-17, the limit of the snap-through regime for all three buckling load values is decreasing until it reaches to its minimum about the first natural frequency of the system, and then starts to increase for higher exciting frequencies. This behavior happens as a result of dumping more energy into the system at the resonance frequency. For $P=35 N$ (Figure 15), the required base vibration for invoking snap-through motion is not very large due to the relatively low compressive load applied to the system, which brings more flexibility to the structure to be able to travel between its two stable states. Here, the frequency at which the minimum base amplitude is required for the snap-through motion is around $22.85 \mathrm{~Hz}$ for the buckling load $P=35 \mathrm{~N}$. By increasing the buckling load to higher values (Figures 16 and 17), the required base vibration for initiating the snap-through motion is significantly raised, as the buckled beam becomes stiffer and makes it harder for the system to travel between its stable states. Note that the frequency at which the minimum required base amplitude for inducing snap-through motion occurs shifts to higher excitation frequencies due to stiffening occurring in the main beam by increasing the buckling load. 


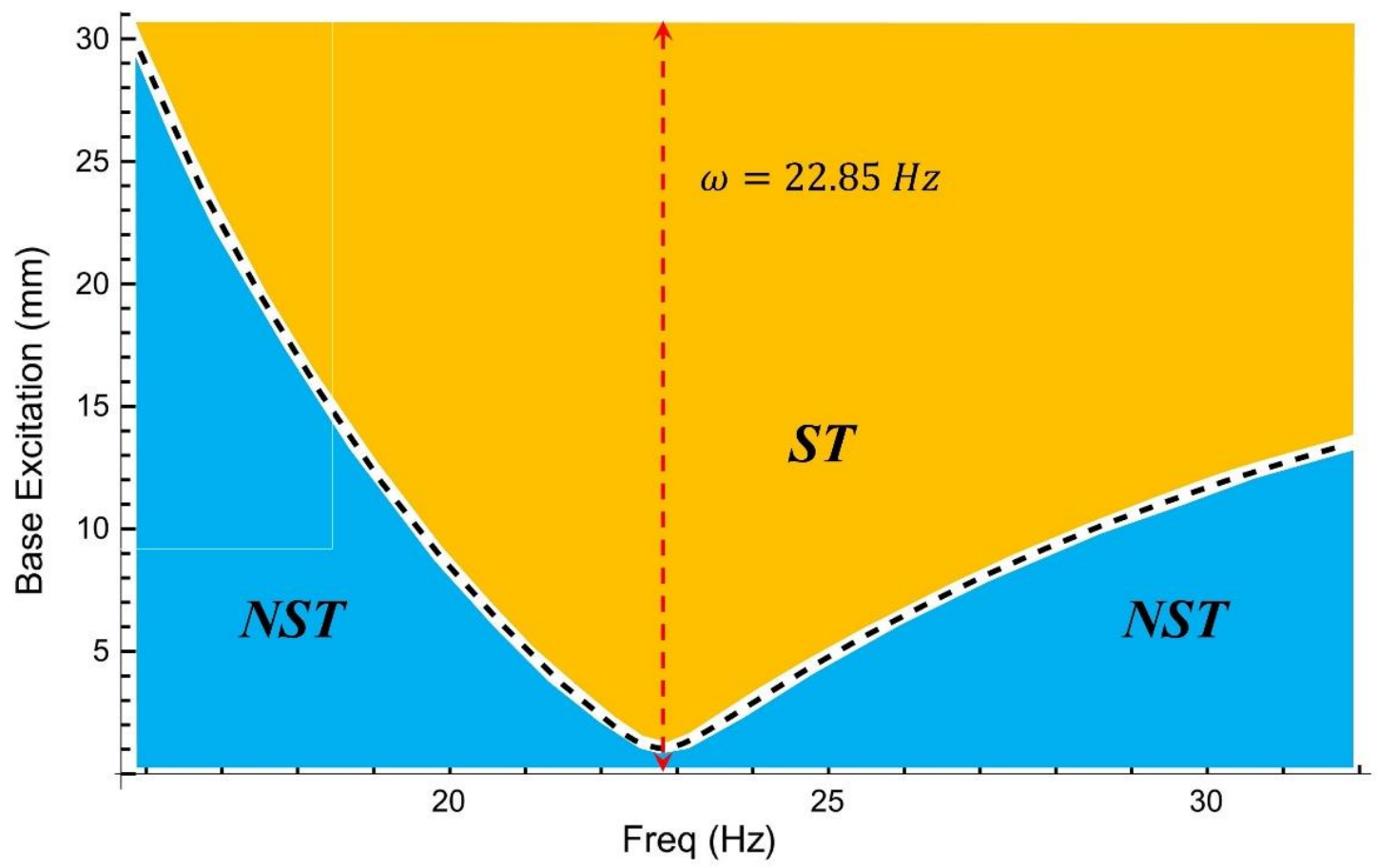

Figure 15. Modeling results of base excitation versus driving frequency showing regimes of snap-through (ST) and no snap-though (NST) behavior for the experimental device with the buckling load $P=35 \mathrm{~N}$. 


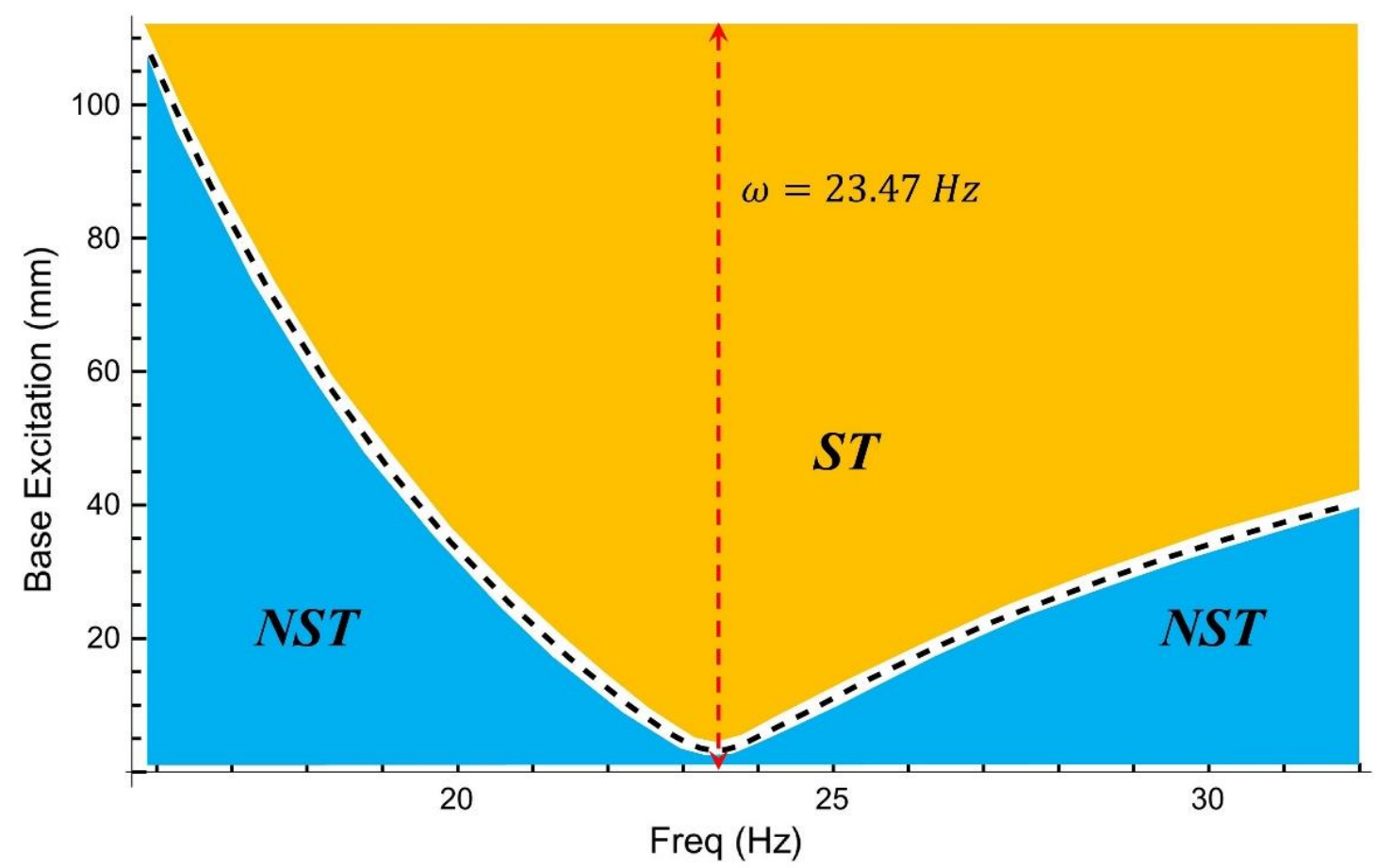

Figure 16. Modeling results of base excitation versus driving frequency showing regimes of snap-through (ST) and no snap-though (NST) behavior for the experimental device with the buckling load $P=45 \mathrm{~N}$. 


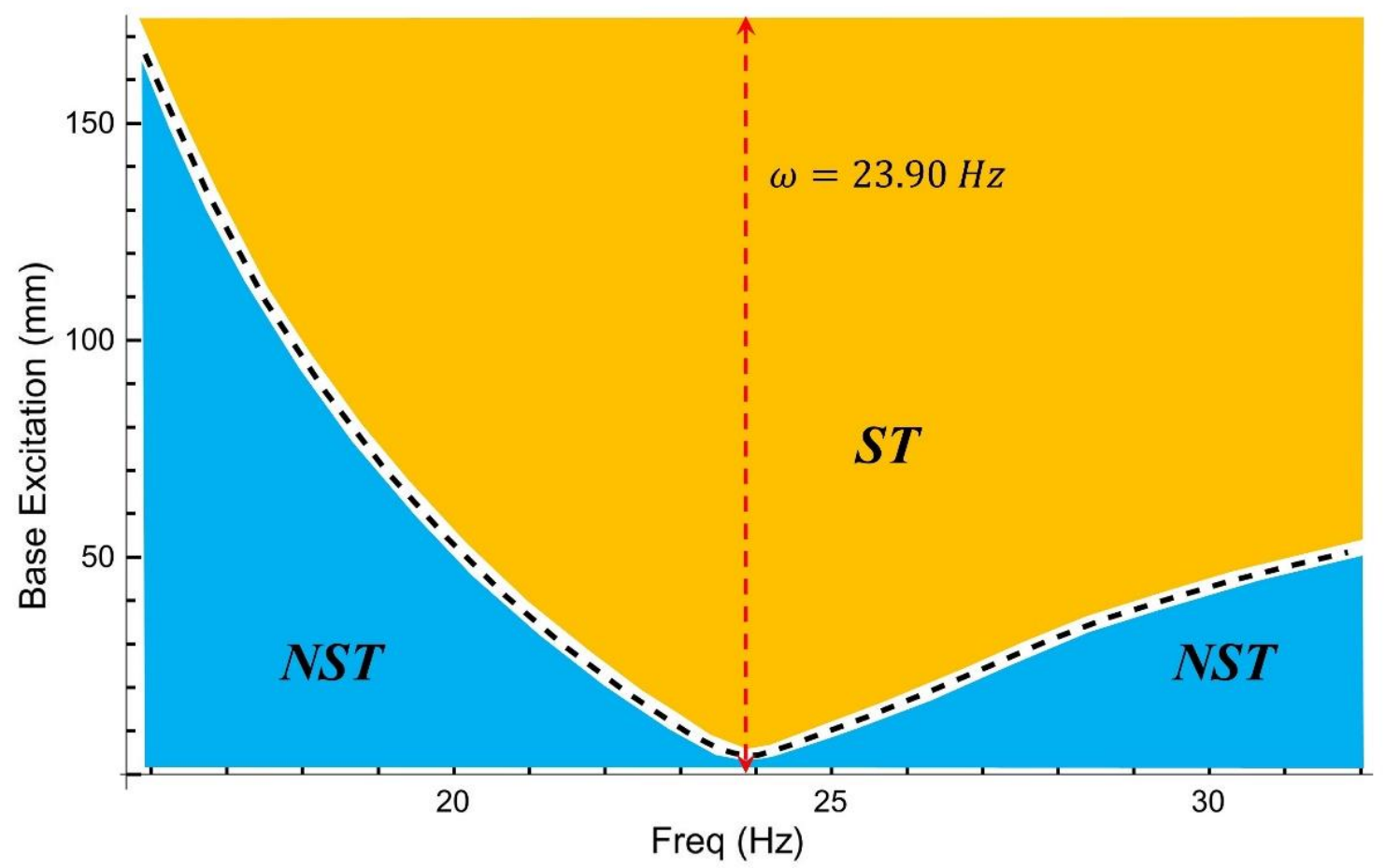

Figure 17. Modeling results of base excitation versus driving frequency showing regimes of snap-through (ST) and no snap-though (NST) behavior for the experimental device with the buckling load $P=55 \mathrm{~N}$.

Figures 18-20 show snap-through regimes for applied buckling loads versus excitation frequency under three different set base vibration amplitudes. The frequency bandwidth at which snap-through motion occurs becomes smaller for all three base amplitudes as the buckling load increases to higher values. As expected from Figures 18-20, this bandwidth becomes wider for higher base vibrations due to greater availability of external energy to the system, which makes it easier to overcome the energy barrier separating the two stable buckled states. It is shown that for the barely-buckled system $\left(P_{c r}=35 \mathrm{~N}\right)$, bistable motion can be obtained for a large exciting frequency bandwidth, behavior that is essential for vibration energy harvesting systems. However, this is not a high energy pay off due to the 
less strain variation in the buckled beam compared to higher buckling forces. This implies a trade-off for energy harvesting applications (harvesting bandwidth versus strain generation during snap-through). Optimizing for applications could be performed by tailoring the central beam buckling load towards the range of available excitation frequencies and driving amplitudes of the harvesting environment.

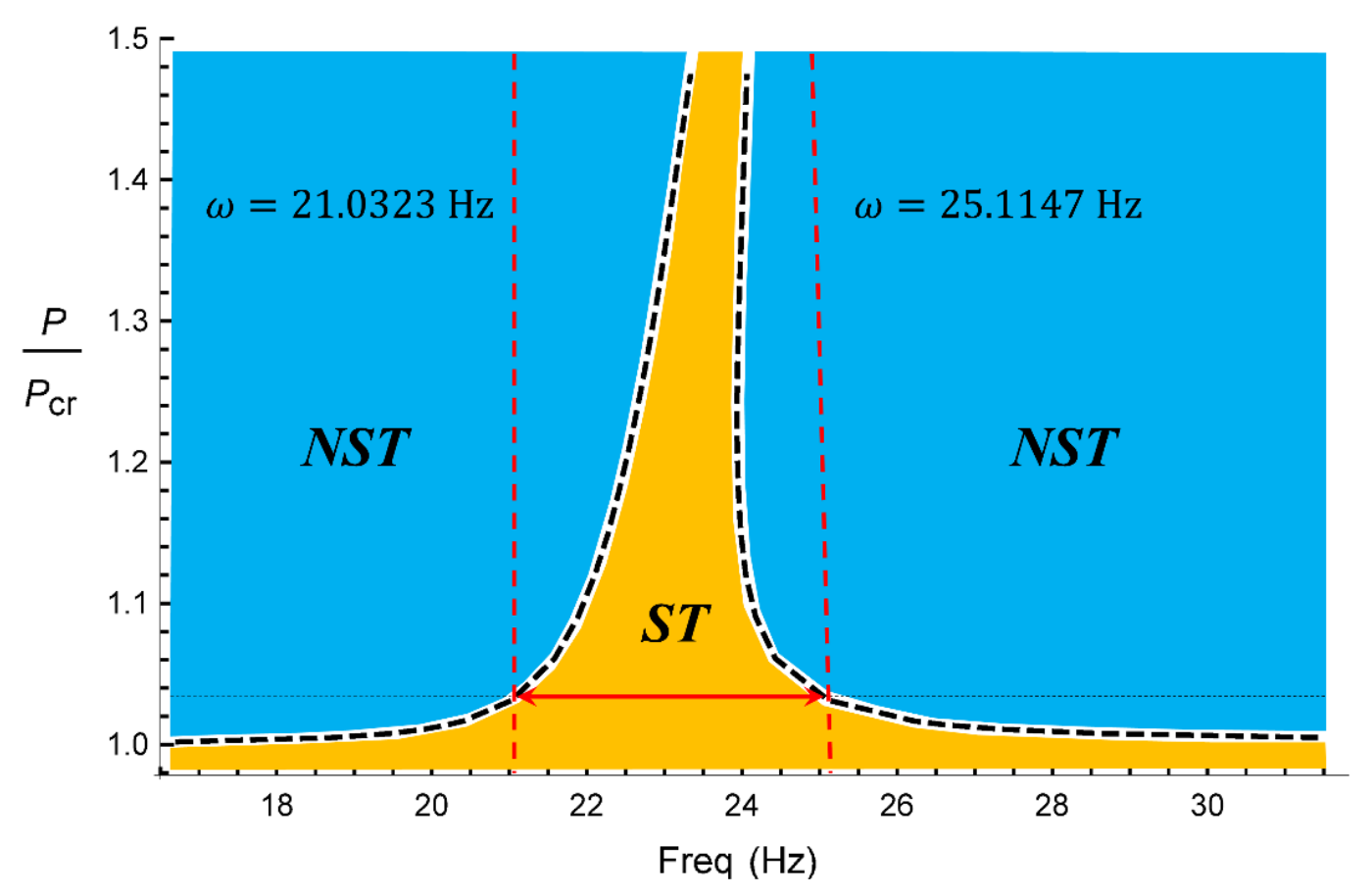

Figure 18. Snap-through regime plot of the experimental device for buckling load vs exciting frequency for harmonic base vibration with the amplitude of $5 \mathrm{~mm}$. 


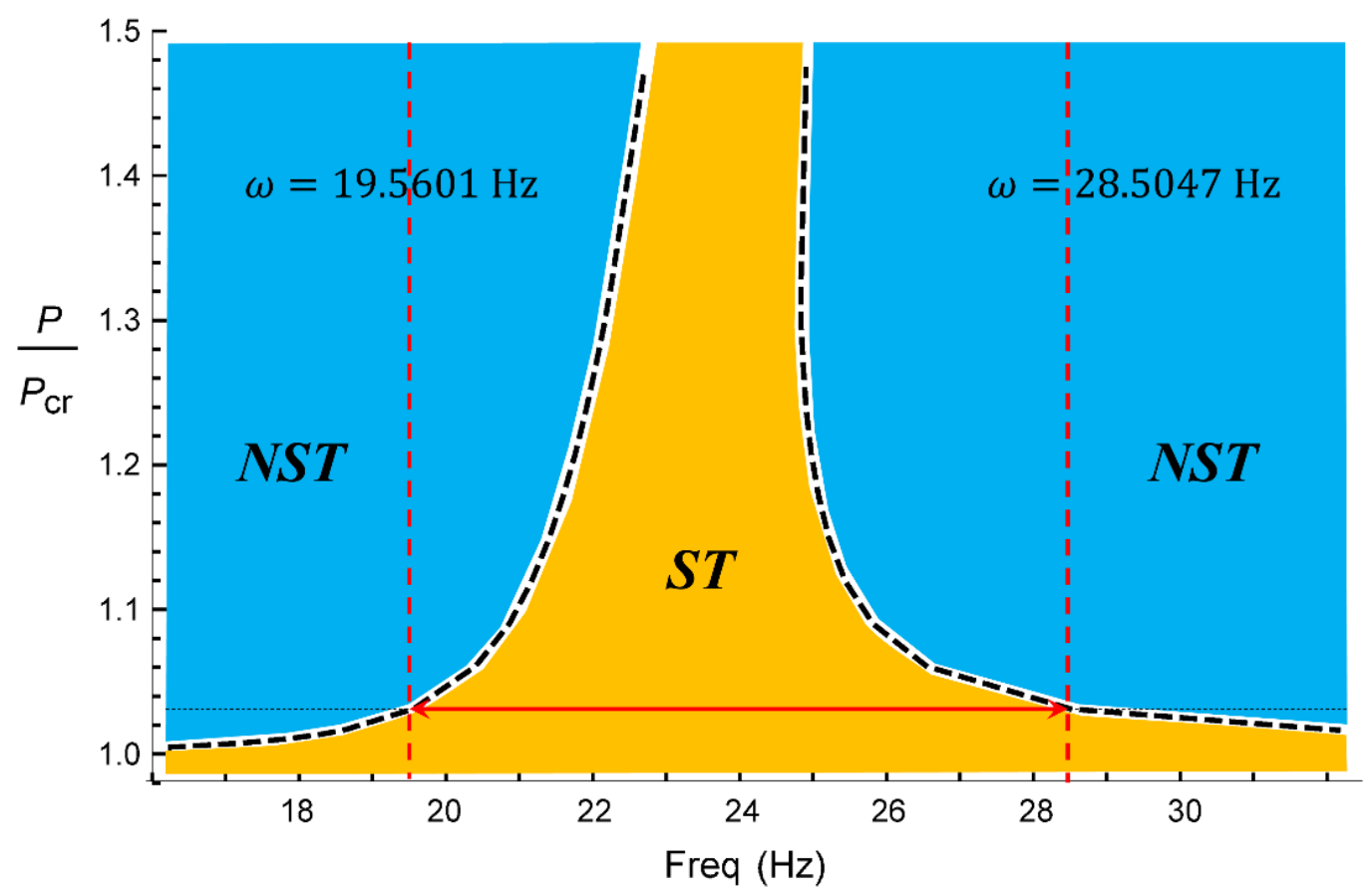

Figure 19. Snap-through regime plot of the experimental device for buckling load vs exciting frequency for harmonic base vibration with the amplitude of $10 \mathrm{~mm}$.

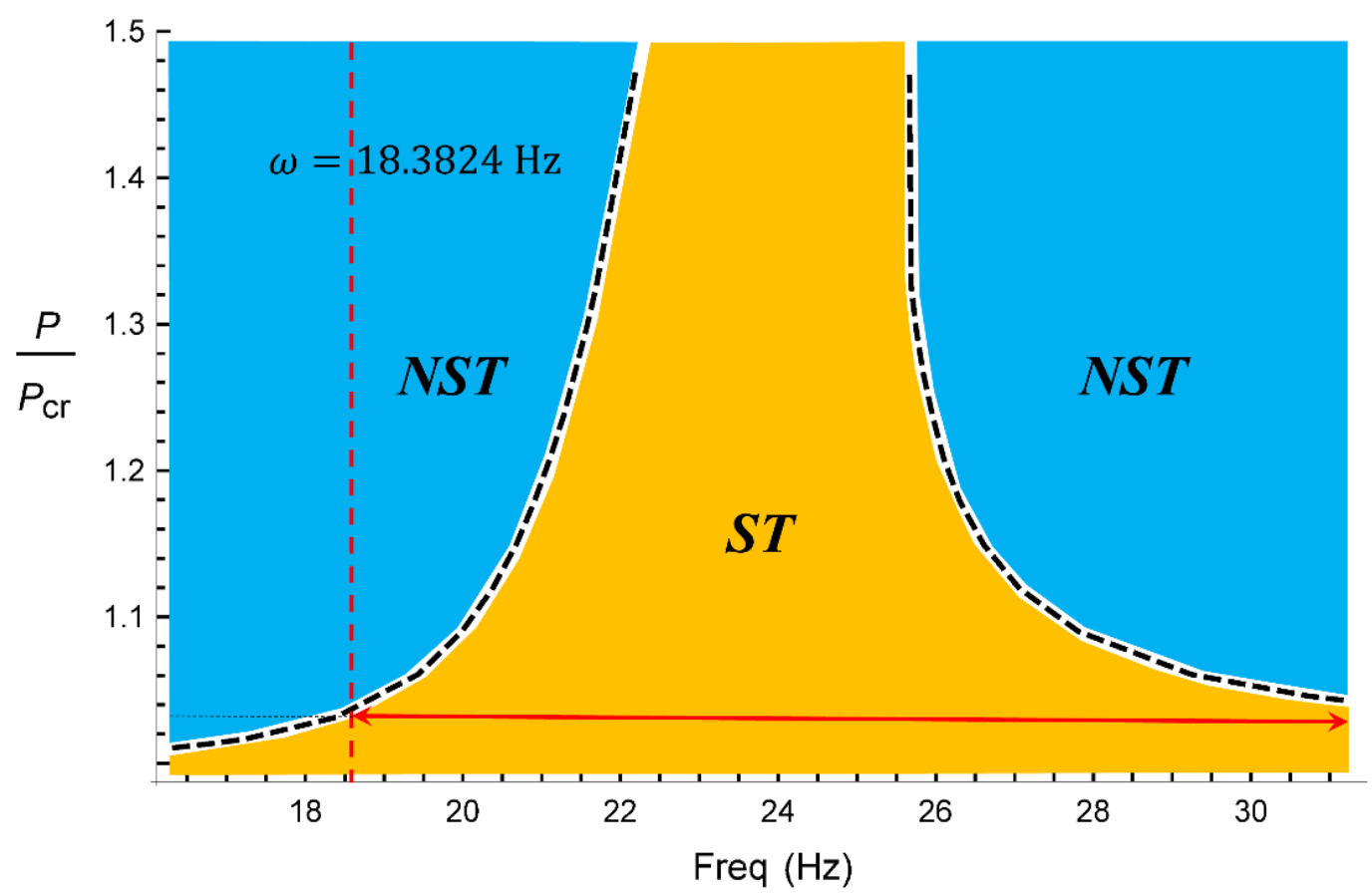


Figure 20. Snap-through regime plot of the experimental device for buckling load vs exciting frequency for harmonic base vibration with the amplitude of $15 \mathrm{~mm}$.

The final modeling result obtained in this study is the estimated snap-through regime of the experimentally tested device for base vibration amplitude versus buckling load at $23.5 \mathrm{~Hz}$ driving frequency, close to the first natural frequency of the system (Figure 21). The base vibration required for inducing snap-through motion increases overall, for higher values of the buckling load. However, the slope of this variation for the buckling levels between 1.15 and 1.35 is almost zero. This is due to the hardening behavior occurring in the system, which results in shifting the maximum vibration amplitude to the higher frequencies (from less than $23.5 \mathrm{~Hz}$ at lower buckling levels to $23.5 \mathrm{~Hz}$ and more at higher buckling levels). This phenomenon makes the system have the same minimum base amplitude required for occurring snap-through motion within a range of buckling levels (from 1.15 to 1.35 for this specific case) at a certain exciting frequency. This is consistent with the results obtained from the experimental test in Figure 6, in which the system was excited around its first natural frequency for different buckling ranges. It was shown that the snap-through motion can be captured for moderately buckled configurations and by increasing the applied compressive load and making the system highly buckled, the structure would not have bistable motion even for relatively high base excitation. Most practical energy harvesting cases will tend to focus on the smaller base excitation ranges shown in Figure 21, which is in the range of structure motions typically targeted for vibration power scavenging. 


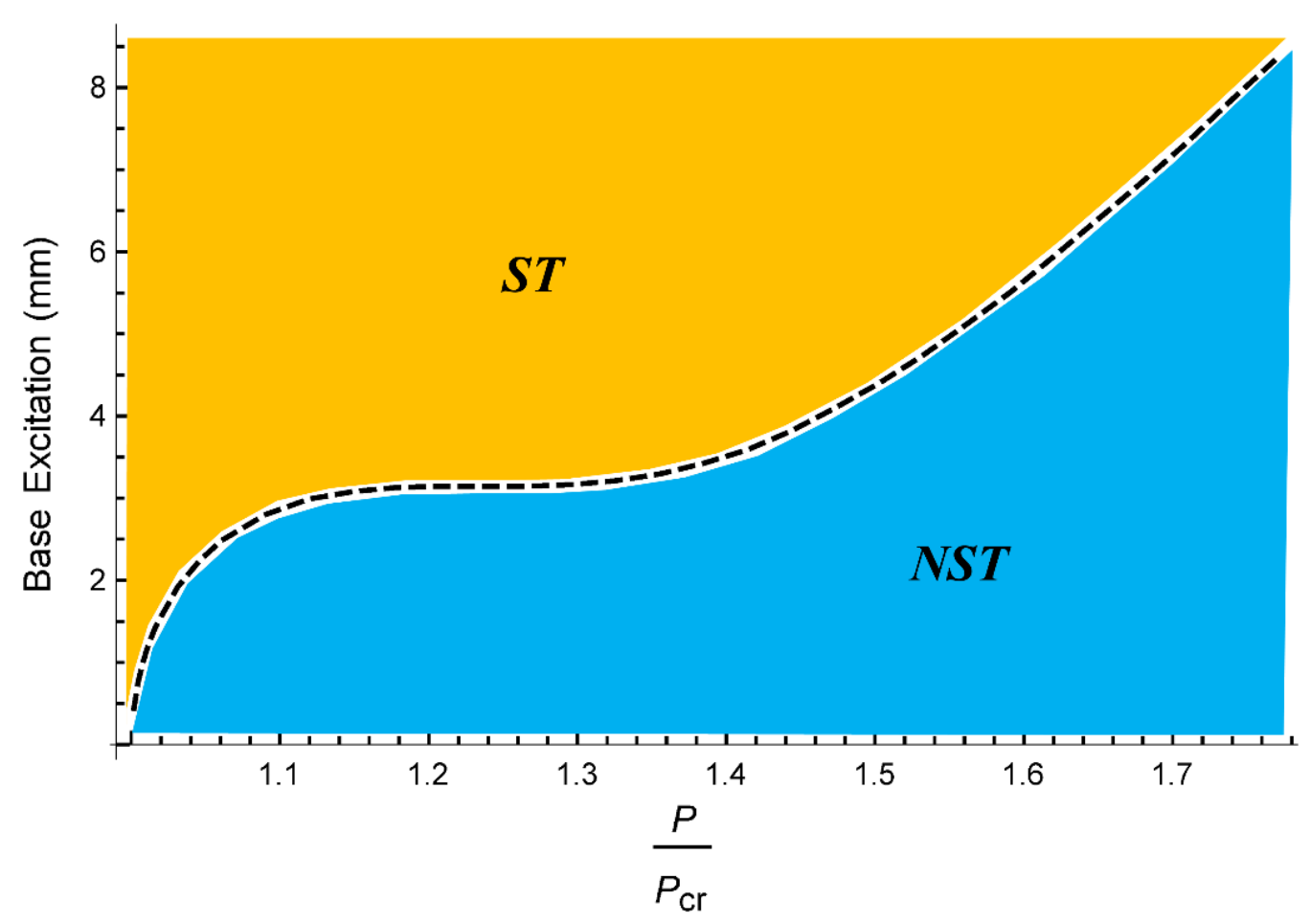

Figure 21. Snap-through regime plot of the experimental device for base vibration amplitude vs buckling load at $23.5 \mathrm{~Hz}$ exciting frequency.

As shown in this chapter, a new approach was implemented to tackle both the static and dynamic analysis of the studied coupled bistable structure for vibration energy harvester. To be able to satisfy all geometrical and force-moment boundary and matching conditions at the connection points, the coupled system was departed into its different components. By solving the static as well as the free vibration of the linearized buckled system, critical buckling load, post-buckled static shape, and linearized natural frequencies and mode shapes of the system were obtained in the modeling section. These obtained mode shapes were used to discretize and consequently get the response of the nonlinear system via a Galerkin discretization. 
One of the highlighted points of this study is the method presented to solve a more sophisticated coupled system, which can be applied to similar multi-component structures and implements all boundary conditions in a manner that produces accurate results for their static and dynamic responses (a different bistable system is studied by this method in chapter six). Furthermore, the results acquired from the model are thoroughly analyzed for the experimental device described in chapter three. It is shown that the snap-through regime can be developed by using the presented dynamic modeling for different parameters of the proposed structure as a vibration energy harvester. It confirms that for a moderately lowbuckled system, the snap-through motion can be captured for a wide range of exciting frequencies around its first dynamic mode, which behavior is essential for energy harvesting systems. These modeling results provide guidelines for optimizing the geometry and buckling state of the proposed device over a range of excitation parameters for use as a vibration energy harvester. 


\section{MECHANICAL ELONGATION AND OUTPUT POWER PREDICTION OF A MEMS SYSTEM}

Previous chapter explains the developed model based on a new component coupling approach to solve the nonlinear dynamic response of the proposed energy harvester. As discussed earlier, this design was originated to work as a MEMS-scale vibration energy harvester as it is hard to capture snap-through and chaotic motions due to the shrinkage of the system size. Therefore, a more realistic MEMS device made of different material layers is introduced in this chapter. First, the formulation is developed for the dynamic behavior and mechanical elongation of the system. Then, using dimensions and material layers of a designed MEMS-scale device, a parametric analysis is performed to investigate the strain development within the piezoelectric layer as related to snap-through behavior of the buckled beam under different dynamic loading conditions. The results show that the initial beam buckling stress and the base excitation amplitude are two major influences on the predicted MEMS vibration energy harvester performance and different aspects of the nonlinear response as well as the mechanical elongation and output power prediction are estimated based on the developed model.

\subsection{Introduction}

As discussed previously, the two cantilever arms with masses concentrated at their ends are connected to the central beam in a way makes the $1^{\text {st }}$ natural frequency easier to tailor 
to a particular vibration environment, separate from the dimensions and compressive stress state of the central beam. The large motion created in these arms is transferred to the main buckled beam through torsional rods, assisting the beam in switching between its stable states. The net result is that bistable motion can be readily facilitated over a range of driving frequency and amplitude combinations. Bistable motion in the central beam is particularly desirable as it produces large strains, boosting scavenged power output. This design is especially useful for MEMS energy harvesters, which typically operate best at frequencies higher than most common real-life application environments (above $200 \mathrm{~Hz}$ ) due to dimensional scaling laws.

\subsection{Modeling Formulation}

Similar to the previous chapter considerations, the electro-mechanical coupling effects of the piezoelectric layer are neglected in the structural modeling. In short, this assumes that the mechanical behavior of the composite structure is dominated by the material properties, regardless of the piezoelectric layer charge state. Justification for this assumption is that the stiffness/deformation response of the combined structure is generally unaffected by even the largest potential electrical charges the structure undergoes. This means that the discretized equations of motion for the MEMS system are the same as the ones obtained for the large-scale size, i.e., equations (121-128). Following the same statement, the coupled system of equations and the nonlinear response of the central buckled beam are equations (133) and (134) respectively.

The response of the (31) plane is considered most critical for producing power from the piezoelectric layer, so analysis of the variation of the axial strain and elongation along the main buckled beam is essential for understanding the performance of this system as an 
effective vibration energy harvester. Based on the nonlinear Euler-Bernoulli beam theory, the axial strain relation for the buckled beam is written as follows:

$$
\epsilon_{x x}(x, z, t)=\left[\frac{\partial U_{1}}{\partial x}+\frac{1}{2}\left(\frac{\partial W_{1}}{\partial x}\right)^{2}\right]-z\left(\frac{\partial^{2} W_{1}}{\partial x^{2}}\right)
$$

As shown in Figure 22, $U_{1}$ and $W_{1}$ are respectively the axial and lateral displacement of the first part of the nonlinear beam. As explained earlier here, analysis of the mechanical strain within the piezoelectric layer of the central beam gives an estimate of the power produced. Thus, the strain variation is calculated for the piezoelectric layer of the main beam at location $z=h_{p}$ as shown in Figure 22.:

$$
\epsilon_{x x}(x, t)=\left[\frac{\partial U_{1}}{\partial x}+\frac{1}{2}\left(\frac{\partial W_{1}}{\partial x}\right)^{2}\right]-h_{p}\left(\frac{\partial^{2} W_{1}}{\partial x^{2}}\right)
$$
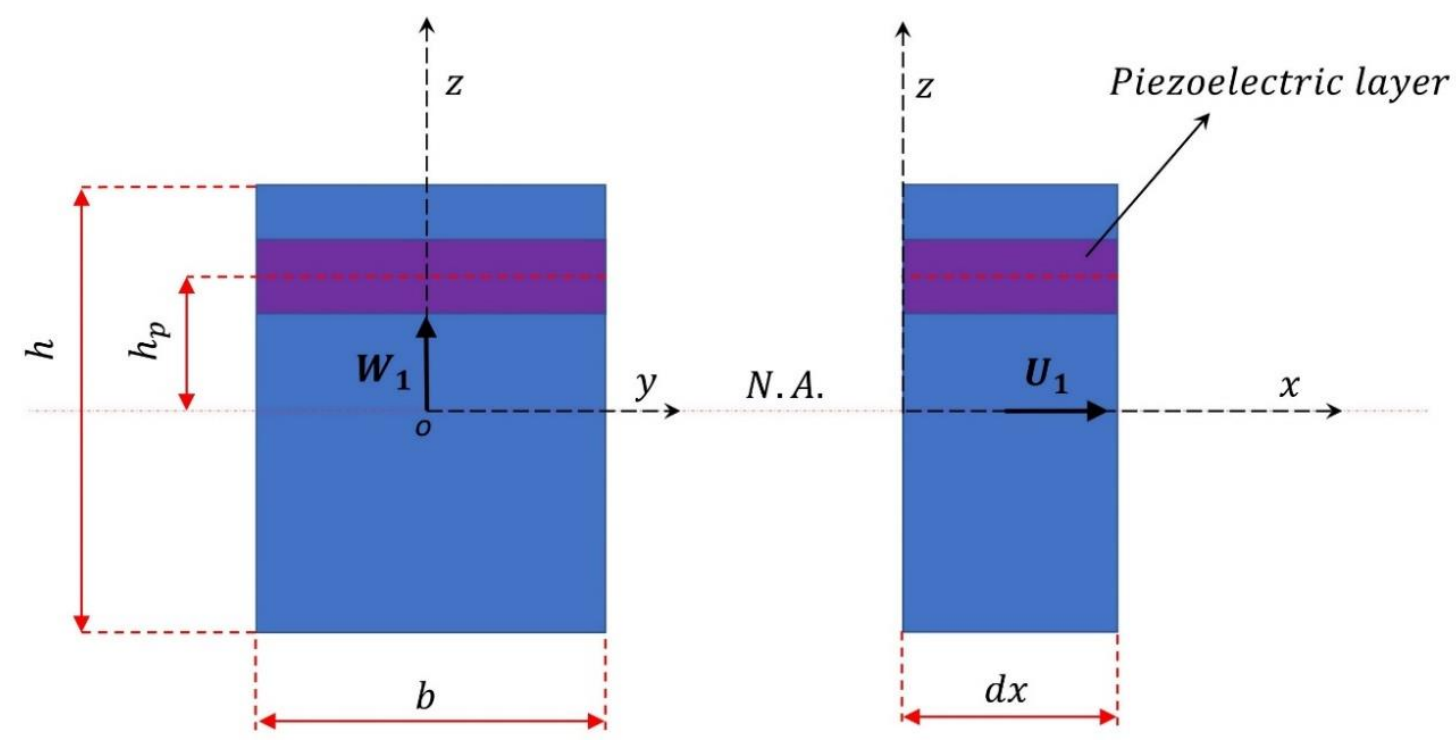

Figure 22. Schematic cross-sectional area and the distance of the piezoelectric layer from the neutral axis. 
Fixed-end ("clamped") beam support conditions are assumed for the post-buckled configuration. For convenience, the entire length of the main beam is considered covered with the piezoelectric layer and electrodes. Therefore, the elongation of the piezoelectric layer of part 1 is formed as follows:

$$
\Delta_{1}(t)=\frac{1}{2} \int_{0}^{\frac{l_{1}}{2}} \epsilon_{x x} d x=\frac{1}{2} \int_{0}^{\frac{l_{1}}{2}}\left(\frac{\partial W_{1}}{\partial x}\right)^{2} d x-h_{p} \int_{0}^{\frac{l_{1}}{2}} \frac{\partial^{2} W_{1}}{\partial x^{2}} d x
$$

Equation (137) indicates that the variation of the axial displacement of the main beam is contributed negligibly to the total elongation. Substituting equation (134) into the obtained elongation relation lead to the following equation:

$$
\Delta_{1}(t)=\frac{1}{2} \int_{0}^{\frac{L_{1}}{2}} W_{1}^{s^{\prime 2}} d x-h_{p} \int_{0}^{\frac{L_{1}}{2}} W_{1}^{s^{\prime \prime}} d x+\sum_{i=1}^{N} \delta_{1}^{i} q_{1}^{i}(t)+\sum_{i=1}^{N} \sum_{j=1}^{N} \delta_{2}^{i j} q_{1}^{i}(t) q_{1}^{j}(t)
$$

where

$$
\delta_{1}^{i}=\int_{0}^{\frac{L_{1}}{2}}\left(W_{1}^{s^{\prime}} \psi_{1}^{i^{\prime}}-h_{p} \psi_{1}^{i^{\prime \prime}}\right) d x, \quad \delta_{1}^{i j}=\frac{1}{2} \int_{0}^{\frac{L_{1}}{2}} \psi_{1}^{i^{\prime}} \psi_{1}^{j^{\prime}} d x ; \quad(i, j=1,2, \ldots N)
$$

$\Delta_{1}(t)$ is the axial mechanical elongation of the first half of the main buckled beam. Similarly, the elongation for the second half of the beam (part 2) is developed as $\Delta_{2}(t)$ and finally, the whole beam elongation could be written as follows:

$$
\Delta(t)=\Delta_{1}(t)+\Delta_{2}(t)
$$

In order to estimate the potential output power of piezoelectric energy harvesters, the constitutive equations for a piezoelectric material are first considered [1]: 


$$
\left\{\begin{array}{l}
D_{z}=d_{31} \sigma_{x x}+\epsilon_{33}^{T} E_{z} \\
\varepsilon_{x x}=d_{31} E_{z}+s_{11}^{E} \sigma_{x x}
\end{array}\right.
$$

where $D_{z}, E_{z}, d_{31}, \epsilon_{33}$, and $s_{11}^{E}$ are electrical displacement, electric field, permittivity, piezoelectric coupling coefficient and elastic compliance of the stacked piezoelectric layer in energy harvester device. By integrating equation (141) over the whole length of the piezoelectric layer, the predicted output charge and power of the bistable energy harvester assuming zero electric field and a purely resistive load are obtained as:

$$
\begin{gathered}
Q(t)=\frac{b d_{31}}{s_{11}^{E}} \Delta(t) \\
P(t)=R \frac{d Q(t)}{d t}
\end{gathered}
$$

Results of the lateral displacement and mechanical elongation of a designed MEMS-scale device utilizing this model are explored in the next section for different harmonic driving excitations, applied buckling stresses, and excitation frequency levels. These cases are used to establish conditions corresponding to both snap-through and non-snap-through regimes. Furthermore, an estimation of the possible output power of the MEMS-energy harvester based on the developed model is performed. The results are then related to the effectiveness of power production for use as an energy harvester.

\subsection{Results and Discussion}

To model a realistic performance analysis of the bistable energy harvester, an actual designed MEMS-scale device was studied for various dimensional and initial condition parameters, as well as typical loading conditions. The experimental fabrication process of such a device will be discussed in chapter seven. Figure 23 shows the top and side view of the analyzing device and dimensions, as well as the direction of the considered base 
excitation. In practice, MEMS-scale devices of this nature feature central beams with an initial buckling stress dictated by the microfabrication process parameters. Thus, this chapter provides dynamic behavior results organized by the initial buckling stress level, rather applied buckling force in the central beam.

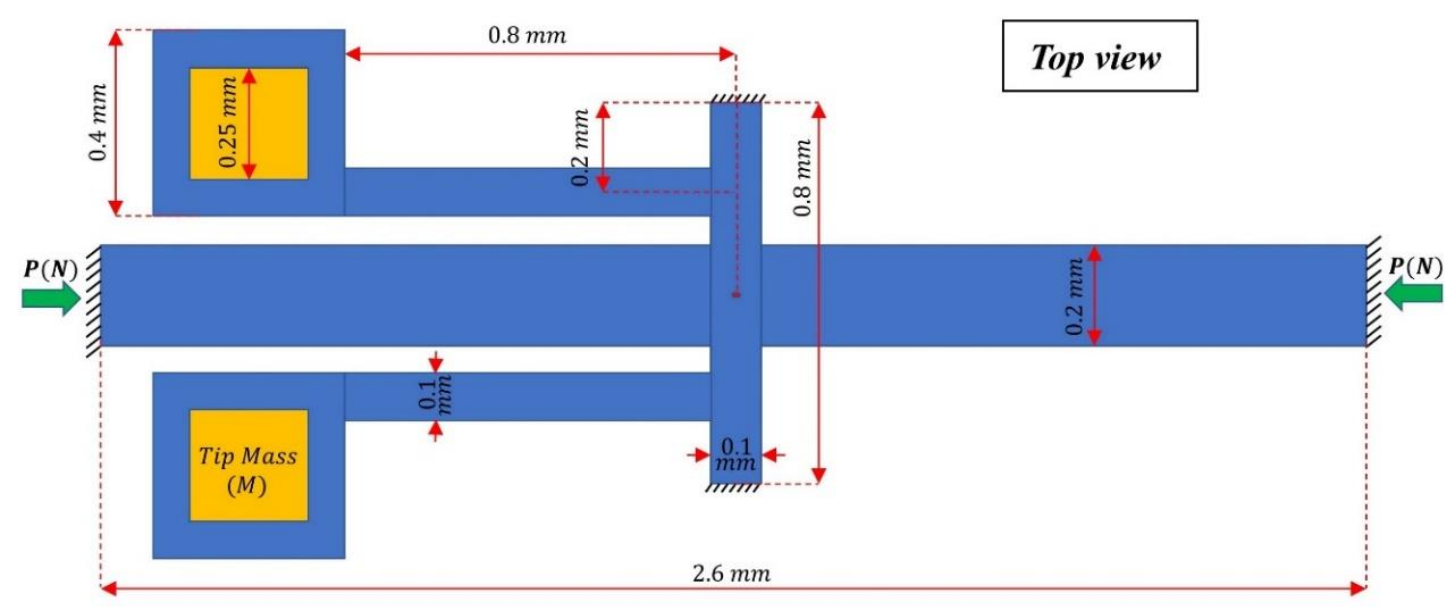

Side view

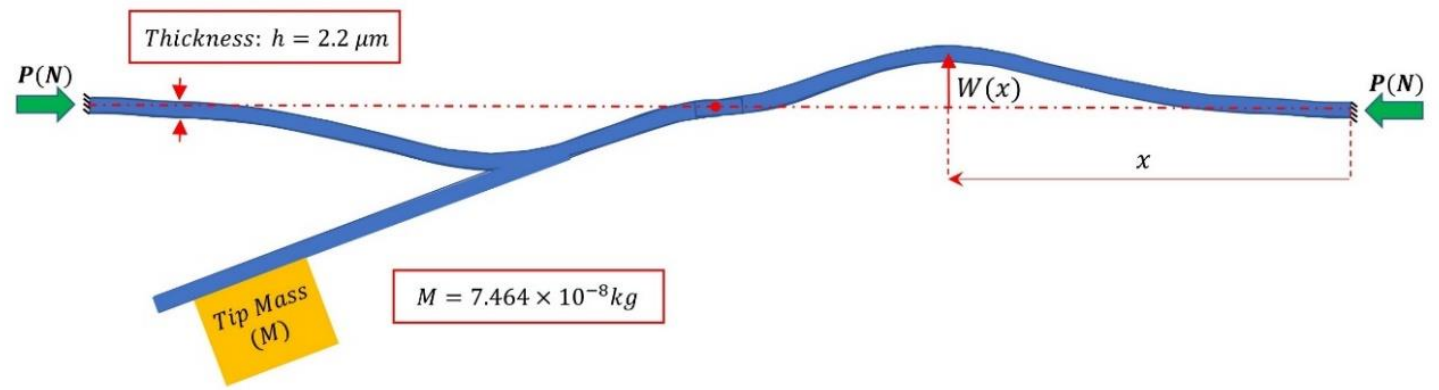


Figure 23. The designed MEMS-scale bistable energy harvester: a) top-view b) sideview.

An actual micro-scale energy harvester consists of different layers including structural, piezoelectric and electrode layers. As explained in the previous section, this formulation considers only the mechanical behavior of the materials included, i.e., the electromechanical coupling components are neglected in the dynamic model. Figure 24 shows the schematic cross-sectional area of the proposed bistable energy harvester. The total height of the device and the thickness of each deposited layer are shown in left side, while the equivalent cross-sectional area of a single modeled layer and its effective modulus of elasticity $E$, Poisson's ratio $v$, and mass per length $\bar{m}$ are shown in the right side of the figure.
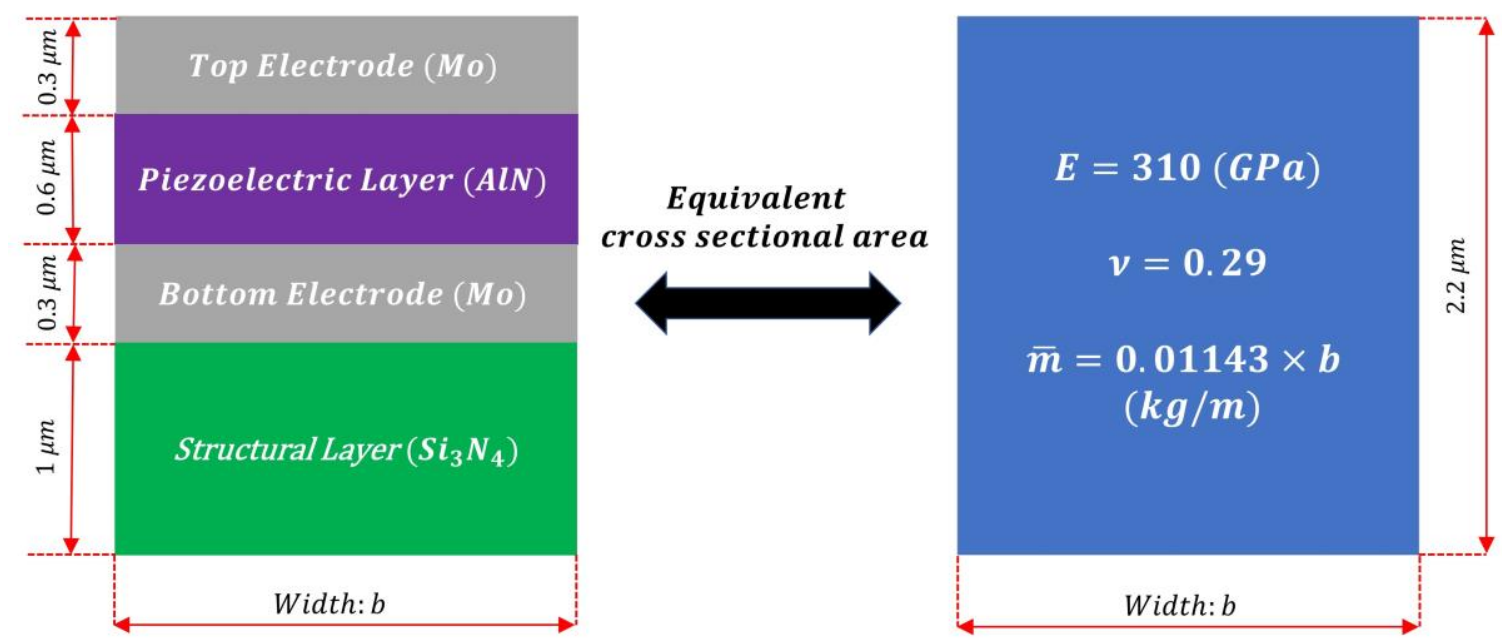

Figure 24. Different layers of the main buckled beam and its equivalent cross-sectional area parameters.

Solving the nonlinear static of the MEMS-device gave the critical buckling loads (or critical buckling stress) and the static mode shapes of the system. The critical buckling 
stress obtained for the described system is $1.93 \mathrm{MPa}$ and its corresponding mode shape is found as shown in Figure 23(b). Moreover, natural frequencies and dynamic modes are found by solving the linearized dynamic equations provided in the modeling section, which requires the given initial buckling stress induced in the structural layer. The first natural frequency of the designed MEMS-scale device with a $2 M P a$ buckling stress level, just above the obtained critical stress, is $115.6 \mathrm{~Hz}$. This resonant frequency is relatively low for comparative micro-scale devices with similar overall size and geometry. Further results show that the first natural frequencies of the device do not change noticeably for higher considered buckling stress levels due to the fact that the system response is dominated by the dynamic behavior of the cantilever arms. The first five natural frequencies of the linearized buckled system are listed in Table 3.

Table 3. First five natural frequencies obtained for the MEMS-scale bistable energy harvester.

\begin{tabular}{|l|c|c|c|c|c|}
\hline Mode shape & Mode 1 & Mode 2 & Mode 3 & Mode 4 & Mode 5 \\
\hline $\begin{array}{l}\text { Natural frequencies } \\
(\mathrm{Hz})\end{array}$ & 115.6 & $7,497.4$ & $1,1381.6$ & $1,3205.4$ & $2,4711.1$ \\
\hline
\end{tabular}

Figure 25 shows a mapping of snap-through regimes of the studied MEMS-scale device for various base amplitudes versus the applied excitation frequency. The results are developed based on the presented modeling approach and assumption of a $1.94 \mathrm{MPa}$ buckling stress, which is just above the critical stress required to produce a bistable system (an optimal configuration for inducing snap-through during vibration driving). Similar to the previous chapter, the first three modes are considered for each part in the Galerkin 
discretization and a harmonic driving motion is assumed for the base excitation. As shown in Fig. 6, the base excitation required for the energy harvester to produce a bistable transition (snap-through motion) decreases with increasing excitation frequency until reaching its minimum at the system's first natural frequency. After passing the resonant frequency, the base excitation required to produce snap-through starts to increase for higher frequencies. This is the same behavior observed in the macro-scale results (chapter four) and it indicates that snap-through motion and the associated higher power output is most feasible about a range of driving excitations near its first natural frequency, with larger base amplitudes enabling a greater range of snap-through producing excitation frequencies. 


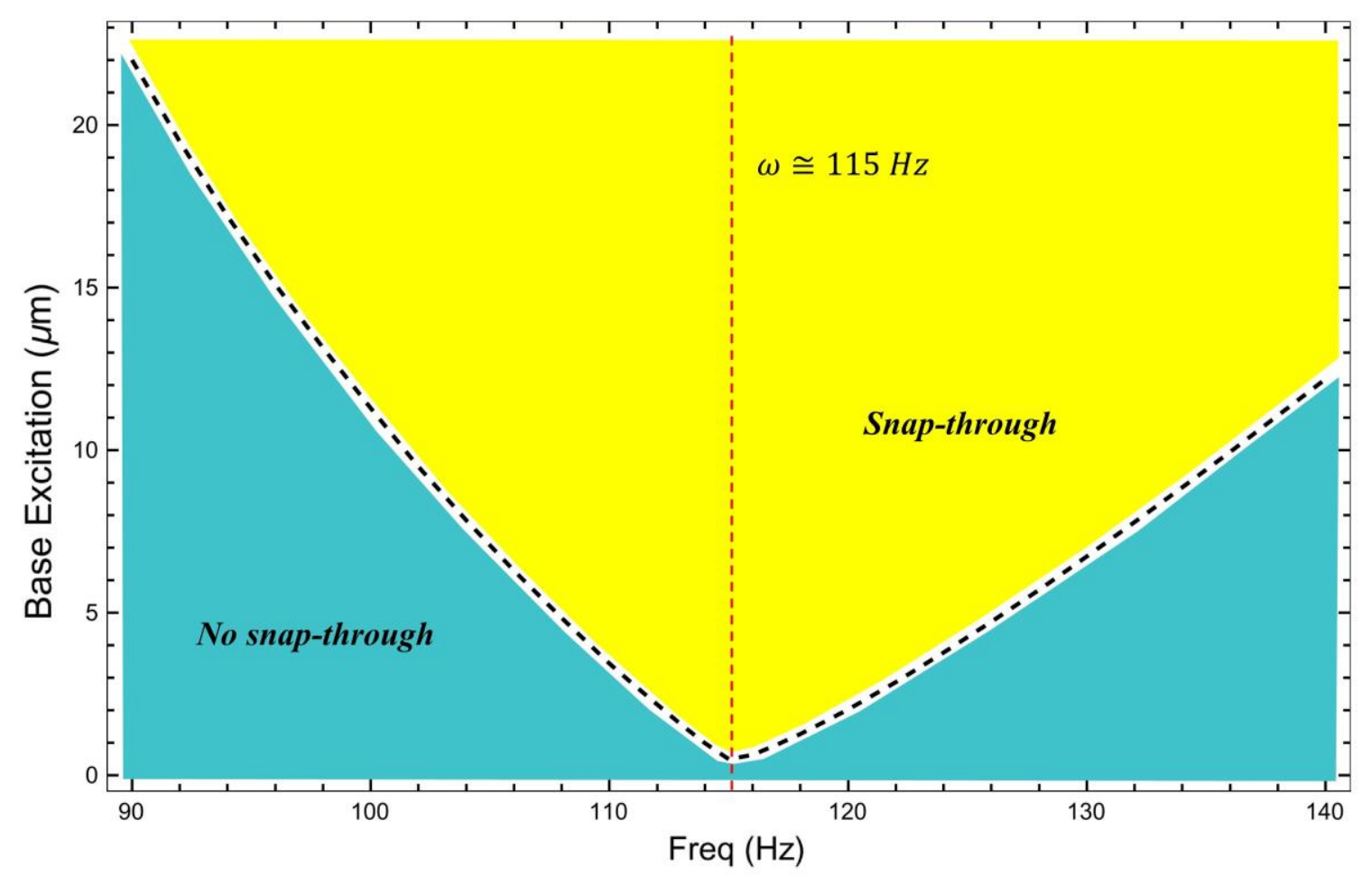

Figure 25. Variation of required base amplitude for the snap-through motion versus exciting frequency.

As the mechanical elongation of the piezoelectric layer in the main beam is directly related to the harvested output power, it is important to know how this elongation changes under various loading situations. Figure 26 shows the variation of the mechanical elongation amplitude of the device part 1 versus buckling stress while driven at an excitation frequency of $115 \mathrm{~Hz}$. For each data point (compressive stress level) shown in Figure 26, the driving base excitation was set at the minimum level required for inducing snap-through behavior (ranged from 1 micron to 16 microns). As can be seen from the figure, the mechanical elongation rises with increased initial buckling stress. However, the rate of this improvement declines as the level of buckling increases due to the system getting stiffer. 


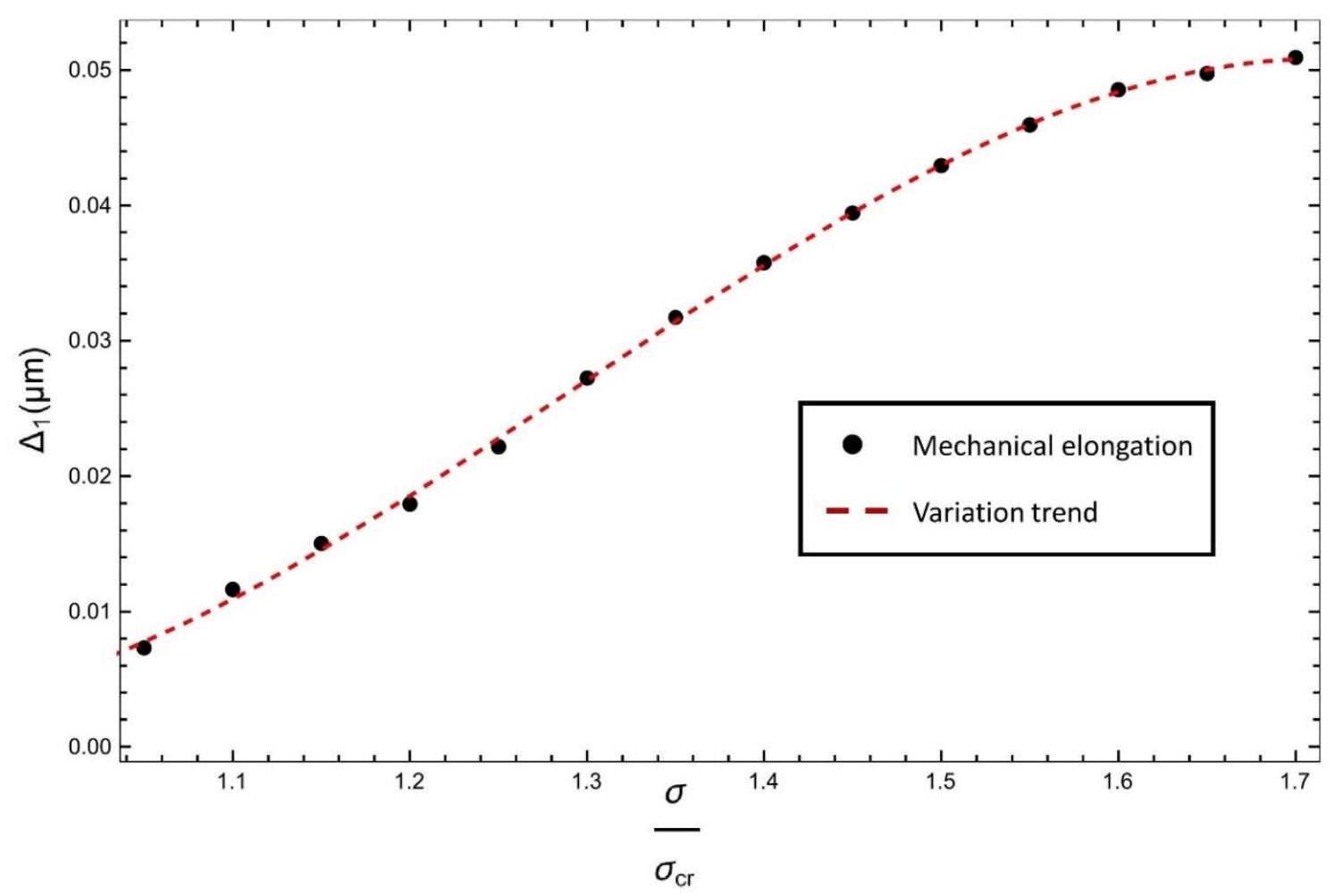

Figure 26. Variation of the main beam elongation amplitude versus central beam compressive stress level (normalized by the critical buckling stress) subjected to vibration sources amplitudes corresponding to the minimum required for bistable motion at each stress level, applied at $115 \mathrm{~Hz}$ excitation frequency.

Mechanical elongation amplitude of the piezoelectric layer in the main buckled beam is modeled for three different initial buckling stresses and a range of driving base excitation amplitudes applied at a frequency of $115 \mathrm{~Hz}$ (Figure 27). As shown for all three buckling levels, the elongation increases gradually when the system vibrates about one of its buckled states, i.e., the system demonstrates monostable motion within a single stable configuration. By increasing the base excitation above a critical level, there is a sudden jump in elongation amplitude for all three cases, which refers to the transition from monostable (local) motions to bistable (snap-through) motions. This illustrates how 
capturing snap-through can improve the harvested power output in the system. The point at which this rapid increase in the elongation occurs shifted with increased buckling stress level. After this abrupt transition, there are modest increases observed in the mechanical elongation for higher driving excitation amplitudes.

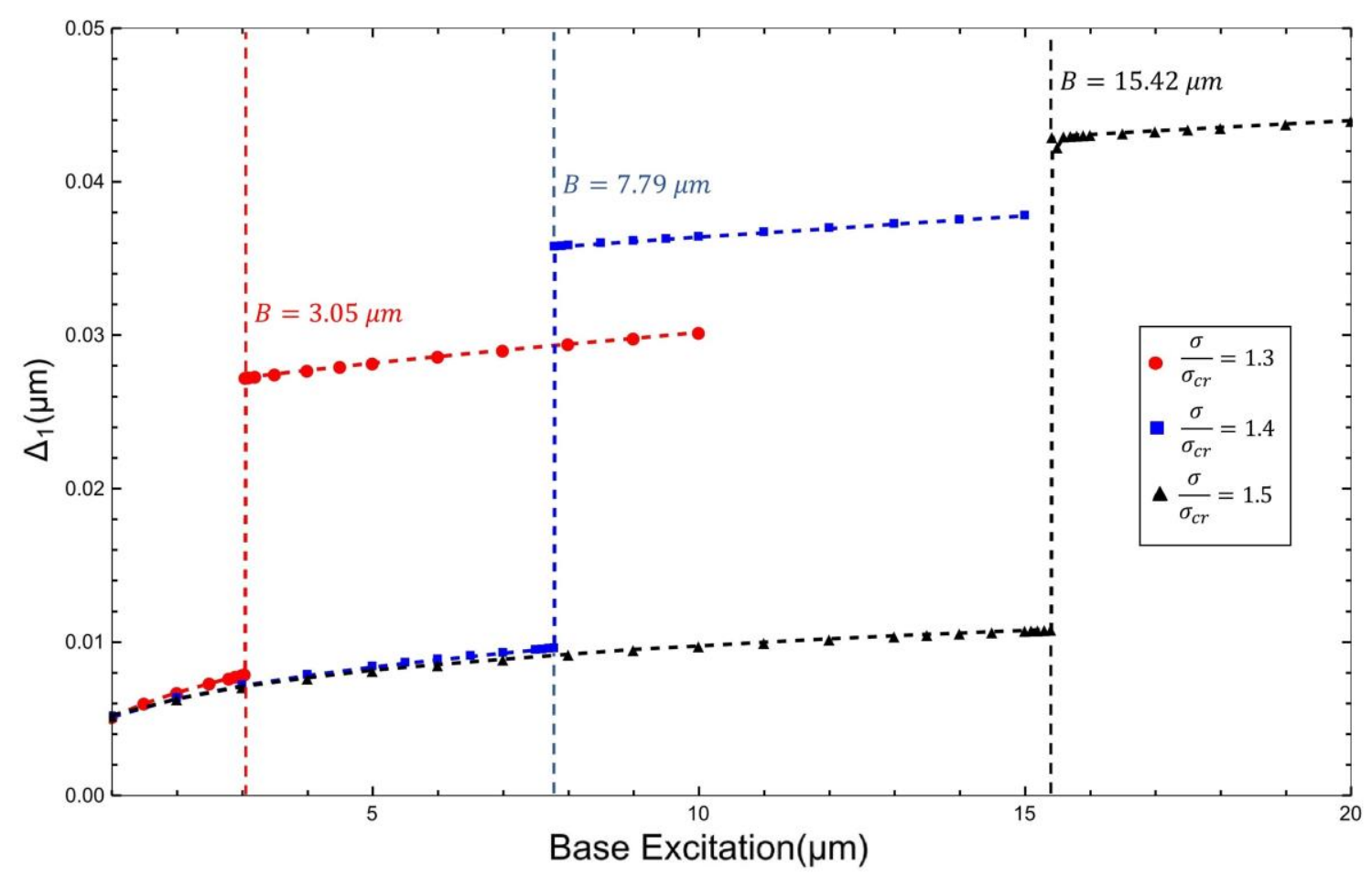

Figure 27. Variation of main beam elongation versus base excitation for three different initial buckling stress levels at $115 \mathrm{~Hz}$ excitation frequency.

To estimate the output power harvested from the piezoelectric layer in the device, the piezoelectric coupling coefficient $\left(d_{31}\right)$ of a fabricated aluminum nitride layer was assumed to be $-3.5 \mathrm{pm} / V$ from [83]. By inserting the aluminum nitride properties into equations (142) and (143), the zero electric field condition charge and harvested power of the MEMSenergy harvester for a purely resistive load are calculated from the model-predicted mechanical elongation of the piezoelectric layer. Two cases of buckling stress level $\sigma=$ 
$1.5 \sigma_{c r}$, and $\sigma=2 \sigma_{c r}$ were considered for the output power estimation. The results shows that the maximum amplitude of the harvested power for $100 \mathrm{~K} \Omega$ external resistive load and $115 \mathrm{~Hz}$ excitation frequency is in the range of $0.05-0.25 \mathrm{pW}$ for $10 \mu \mathrm{m}$ amplitude of base excitation which makes the device have only local or monostable motion. However, this harvested power can increase to $0.2-1 \mathrm{nW}$ for $1 \mathrm{~mm}$ base amplitude (a more realistic condition for an actual device) in which snap-through and chaotic motions occurrs. These results are provided to show an estimation of harvested power produced by the two configurations of the system described in Figure 22. Improved power output could be gained through selection of materials and dimensional parameters optimized to a particular vibration source.

The last figure shows in this chapter is a regime plot of snap-through behavior for the micro-scale device for initial buckling stress versus driving excitation frequency (Figure 28). These results are obtained for $20 \mu m$ amplitude of base excitation. As shown in the figure, the frequency bandwidth within which snap-through is predicted gets narrower as the buckling stress increases. Thus, the primary benefit of "just" buckled systems is broadband performance as an energy harvester. However, the mechanical elongation and associated output power captured for these low initial buckling stress cases are outperformed by higher initial buckled cases, provided that the driving excitation amplitude is sufficient to induce bistable motion. These results indicate optimum energy harvesting power output for this bistable device requires a need to tailor the initial buckling stress parameter within the fabricated layers to both the operating frequency bandwidth and the driving amplitude. 


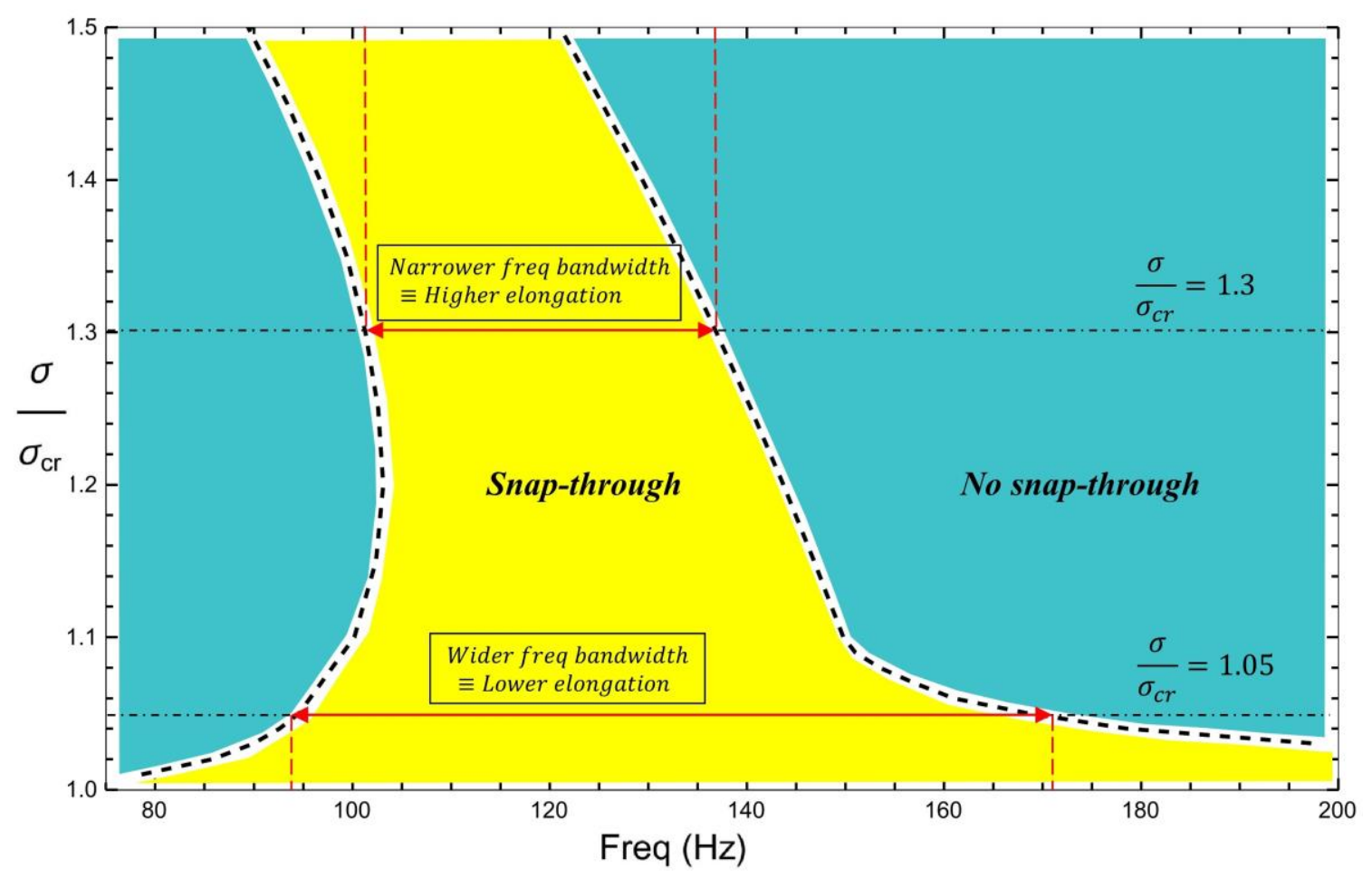

Figure 28. Snap-through regime plot of the MEMS-device for buckling stress versus exciting frequency.

The dynamic analysis of the micro-scale bistable energy harvester developed in this chapter shows that initiation of snap-through switching of the central buckled beam creates a dramatic increase in the strain elongation of the piezoelectric layer and consequently the harvested power of the device. It is also found that central beams with initial buckling stress just above the critical level produce devices with the broader band of operating frequencies viable for power production; however, adjusting this induced stress close to the critical level is barely practical in the experimental fabrication process. Further strain potential and harvested power could be gained with even larger initial buckling stress levels, though at a sacrifice to operation frequency range, provides that sufficient driving amplitude must be supplied to induce snap-through motion. Optimal vibration energy scavenging capability 
requires balancing the trade-off between these factors associated with the targeted vibration source. 


\section{ANALYSIS OF A PVDF-BASED CLAMPED-CLAMPED BUCKLED STRUCTURE}

As shown in the previous chapters, the developed component coupling approach can deliver a more accurate dynamic response of the proposed coupled structure due to the satisfaction of all geometric and force-moment boundary conditions at the connection points. One of the advantages of this method is its generality, which means it can be applied to other coupled nonlinear structures. For this purpose, a clamped-ends buckled beam made of piezoelectric material as its structural layer with a central mass in the middle is dynamically modeled in this chapter. Polyvinylidene fluoride (PVDF) is considered as the piezoelectric layer and its dynamic results are compared with the ones obtained from the experimental tests. The results show the robustness of this coupling approach especially for the estimation of snap-through and chaotic motions, which are essential to predict in vibration energy harvesting applications.

\subsection{Introduction}

This chapter presents an in-depth analysis of the nonlinear dynamic response of a bistable, buckled structure utilizing polarized polyvinylidene fluoride (PVDF) to perform as a vibration energy harvesting system. The stability and dynamic analysis of the structure under vibration loading is performed by the presented coupling component method and the resulting output voltage is analytically predicted and compared with the data obtained from 
the experimental setup. Contrary to the previous chapters where the main structure of the system did not have piezoelectric properties and therefore only the mechanical governing equations were considered, here, the coupled electrical-mechanical equations of motion for a geometrically nonlinear Euler-Bernoulli piezoelectric beam are developed via Hamilton's principle. As required by the component coupling approach, the system is divided into its components in a way that the effect of connection is considered as matching conditions between parts, which allows one to exactly solve the buckled static and linearized dynamic system and eventually have a more accurate analysis of the nonlinear response. This method is used to analyze both the displacement and voltage responses for the described system. In particular, the regimes of buckled state switching corresponding to higher power production are predicted by the model. As mentioned above, to have a better understanding of the modeling results, an experimental setup of the described device made of commercial PVDF sheet has been tested on a custom-built shaker. The results show a relatively good agreement between the developed model and the experiment, which in turn prove the presented coupling approach capability of predicting the dynamic behavior of the studied bistable system.

\subsection{Modeling and Formulation}

The energy harvesting system considered in this chapter is a clamped-clamped buckled beam with a concentrated mass in the middle (Figure 29). The beam contains a core of polyvinylidene fluoride (PVDF), a polymer with piezoelectric properties. The beam is initially buckled by applying a longitudinal compressive displacement to one clamped end of the beam. A concentrated mass is added to the center point of the buckled beam to lower 
the first natural frequency of the energy harvester and help promote snap-through motions between stable buckled states at the desired low-frequency range.

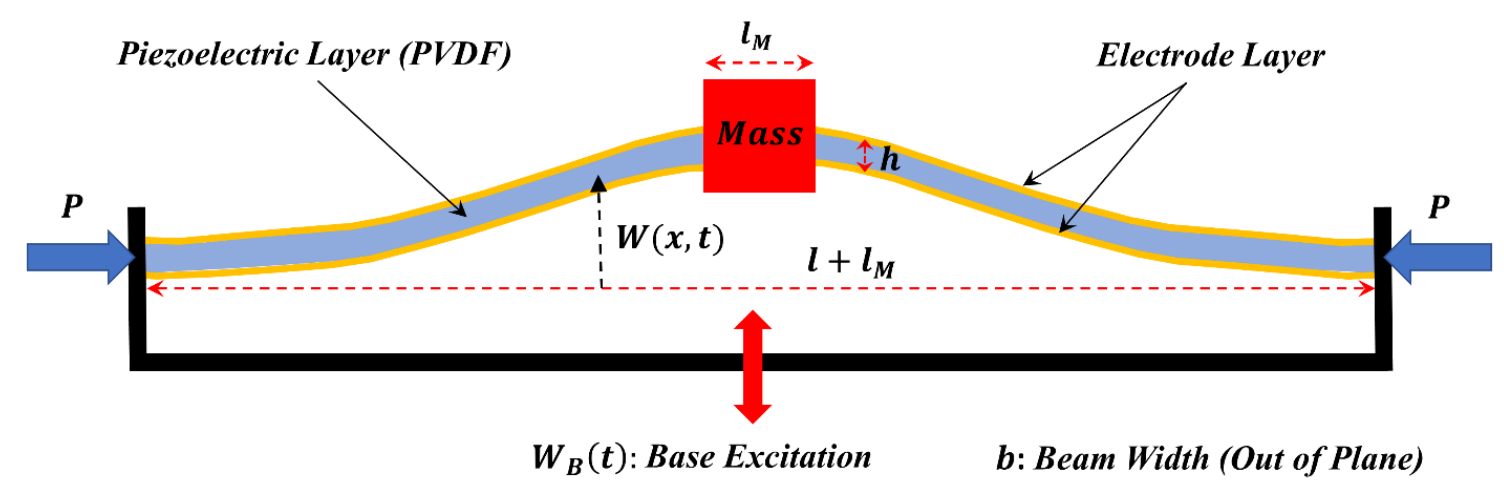

Figure 29. Side view of the PVDF-based, buckled bistable system for vibration energy harvesting.

As the bulk of the beam structure is made of PVDF (top and bottom Cu electrodes both < $10 \mu \mathrm{m})$, the constitutive relationships for piezoelectric materials are considered in generating the equations of motion. Applying Hamilton's principle delivers the following coupled differential equations for a nonlinear Euler-Bernoulli piezoelectric beam with the parametric dimensions shown in Figure 29.

$$
\begin{gathered}
\bar{m} \frac{\partial^{2} W}{\partial t^{2}}+E I_{y} \frac{\partial^{4} W}{\partial x^{4}}+P \frac{\partial^{2} W}{\partial x^{2}}+C \frac{\partial W}{\partial t} \\
-\frac{E A}{2 l} \frac{\partial^{2} W}{\partial x^{2}}\left[\int_{0}^{l}\left(\frac{\partial W}{\partial x}\right)^{2} d x+\frac{b d_{31} E}{l} \int_{0}^{l} v(x, t) d x\right] \\
-\frac{d_{31} E A}{2} \frac{d^{2} v(x, t)}{d x^{2}}=-\bar{m} \frac{\partial^{2} W_{B}}{\partial t^{2}}
\end{gathered}
$$




$$
\begin{gathered}
v(x, t)-\frac{d_{31}^{2} E}{\epsilon_{33}^{T} l} \int_{0}^{l} v(x, t) d x+\frac{h^{2} d_{31} E}{2 \epsilon_{33}^{T}} \frac{\partial^{2} W}{\partial x^{2}}-\frac{h d_{31} E}{2 \epsilon_{33}^{T} l} \int_{0}^{l}\left(\frac{\partial W}{\partial x}\right)^{2} d x \\
+\frac{h}{b \epsilon_{33}^{T}} q(x, t)=0
\end{gathered}
$$

where $W, W_{B}, v$, and $q$ are the beam transverse deflection, base excitation, voltage across the beam, and the electrical charge density $(\mathrm{c} / \mathrm{m})$ respectively. Parameters $\bar{m}, E, I_{y}, d_{31}, \epsilon_{33}, A, C, P$ are mass per length, modulus of elasticity, second moment of area about the out-of-plane axis, piezoelectric constant, electrical permittivity, cross-section area, damping coefficient, and buckling load, respectively.

The lumped mass in the center brings structural discontinuity to the system, which necessitates division of the device into its sub-components to accurately solve the buckled static and linearized dynamics of the system. As is such, the beam is divided into two parts as shown in Figure 30 which effectively allows the central mass to be considered in the boundary conditions. As discussed in chapter four, this selection permits one to exactly solve the nonlinear static and linearized dynamic equations by applying all the geometrical and force-moment boundary conditions. Consequently, it produces a more accurate nonlinear analysis of the bistable device via Galerkin's discretization than traditional techniques. 


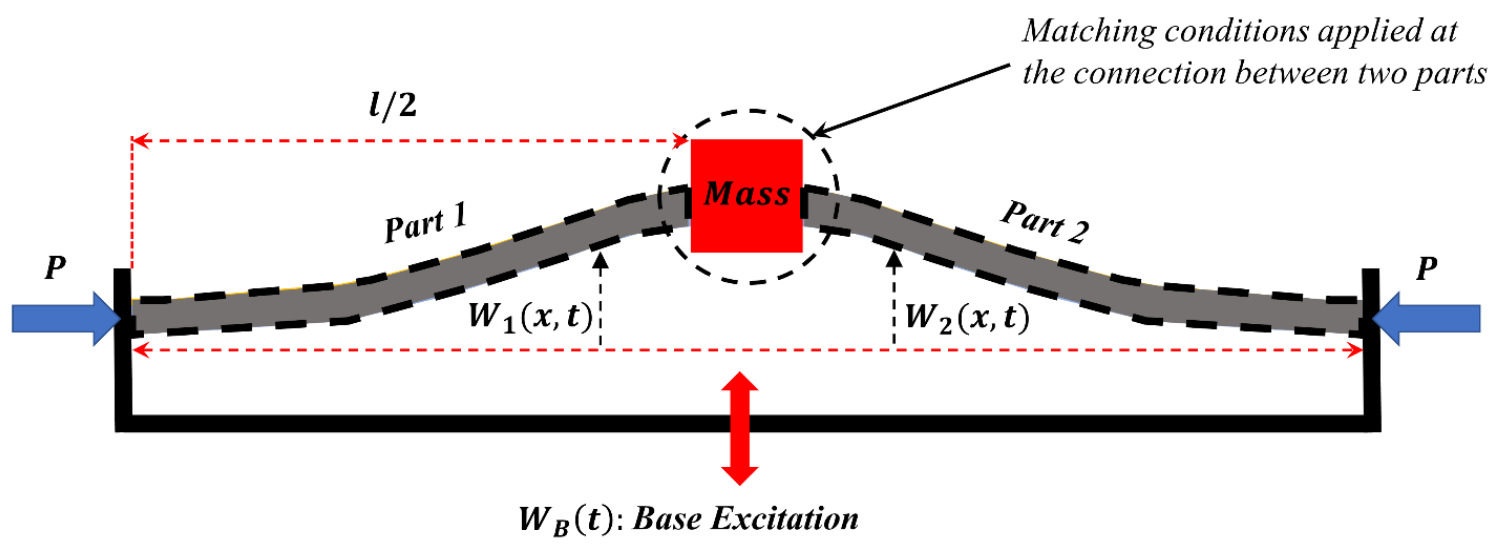

Figure 30. Schematic view of the whole system divided into its subcomponents.

\subsubsection{Static Analysis}

The first step of the solution process is to find the structural shape functions of the system, so the structural equation (144) is rewritten for each part of the system as follows:

$$
\begin{gathered}
\ddot{W}_{1}+\bar{K}_{1} W_{1}^{\prime \prime \prime \prime}+\bar{P} W_{1}^{\prime \prime}+\bar{C} \dot{W}_{1}-W_{1}^{\prime \prime}\left(\bar{K}_{2} \int_{0}^{\frac{l}{2}}{W_{1}^{\prime 2}}^{2} d x+\bar{\alpha}_{2} \int_{0}^{\frac{l}{2}} v_{1}(x, t) d x\right) \\
-\bar{\alpha}_{1} \frac{d^{2} v_{1}(x, t)}{d x^{2}}=-\ddot{W}_{B}, \quad\left(0<x<\frac{l}{2}\right) \\
\ddot{W}_{2}+\bar{K}_{1} W_{2}^{\prime \prime \prime \prime}+\bar{P} W_{2}^{\prime \prime}+\bar{C} \dot{W}_{2}-W_{2}^{\prime \prime}\left(\bar{K}_{2} \int_{\frac{l}{2}}^{l}{W_{2}^{\prime}}^{2} d x+\bar{\alpha}_{2} \int_{\frac{l}{2}}^{l} v_{2}(x, t) d x\right) \\
-\bar{\alpha}_{1} \frac{d^{2} v_{2}(x, t)}{d x^{2}}=-\ddot{W}_{B}, \quad\left(\frac{l}{2}<x<l\right)
\end{gathered}
$$

where

$$
\begin{gathered}
()^{\prime}=\frac{\partial}{\partial x}, \quad\left({ }^{\prime}\right)=\frac{\partial}{\partial t}, \quad \bar{K}_{1}=\frac{E I_{y}}{\bar{m}}, \quad \bar{C}=\frac{C}{\bar{m}}, \quad \bar{P}=\frac{P}{\bar{m}}, \quad \bar{K}_{2}=\frac{E A}{\bar{m} l} \\
\bar{\alpha}_{1}=\frac{E A d_{31}}{2 \bar{m}}, \quad \bar{\alpha}_{2}=\frac{2 b d_{31} E}{\bar{m} l}
\end{gathered}
$$


To find the static buckled shape and critical buckling load, the static equation of motion must be solved. To do so, all the dynamic, damping and voltage terms are dropped from equations (146) and (147), which results in:

$$
\begin{aligned}
& \bar{K}_{1} W_{1}^{\prime \prime \prime \prime}+\left(\bar{P}-\bar{\Gamma}_{1}\right) W_{1}^{\prime \prime}=0 ; \quad\left(\bar{\Gamma}_{1}=\bar{K}_{2} \int_{0}^{\frac{l}{2}}{W_{1}^{\prime 2}}^{2} d x\right) \\
& \bar{K}_{1} W_{2}^{\prime \prime \prime \prime}+\left(\bar{P}-\bar{\Gamma}_{2}\right) W_{2}^{\prime \prime}=0 ; \quad\left(\bar{\Gamma}_{2}=\bar{K}_{2} \int_{\frac{l}{2}}^{l}{W_{2}^{\prime 2}}^{2} d x\right)
\end{aligned}
$$

Due to the geometrical symmetry existing in the static shape of the main beam, $\bar{\Gamma}_{1}$ and $\bar{\Gamma}_{2}$ are equal, and for simplicity are considered as just $\bar{\Gamma}$. Based on this assumption, the general solutions for static differential equations of parts 1 and 2 are obtained as follows:

$$
\begin{aligned}
& W_{1}^{s}=A_{1}^{s} \sin \left(\beta_{s} x\right)+B_{1}^{s} \cos \left(\beta_{s} x\right)+C_{1}^{s}+D_{1}^{s} x \\
& W_{2}^{s}=A_{2}^{s} \sin \left(\beta_{s} x\right)+B_{2}^{s} \cos \left(\beta_{s} x\right)+C_{2}^{s}+D_{2}^{s} x
\end{aligned}
$$

where the superscript " $s$ " refers to the static terms and the coefficient $\beta_{s}$ is described as

$$
\beta_{s}{ }^{2}=\frac{\bar{P}-\bar{\Gamma}}{\overline{K_{1}}}
$$

The presumed static solutions consist of 8 unknown coefficients in total, which can be found from applying all the structural end boundaries as well as matching conditions in the middle connection. These conditions are obtained as the by-products from Hamilton's principle as follows: 


$$
\begin{gathered}
\left\{\begin{array}{c}
W_{1}(0)=0 ; \quad W_{1}^{\prime}(0)=0 \\
W_{2}(l)=0 ; \quad W_{2}^{\prime}(l)=0
\end{array}\right. \\
W_{1}\left(\frac{l}{2}\right)=W_{2}\left(\frac{l}{2}\right) \\
W_{1}^{\prime}\left(\frac{l}{2}\right)=W_{2}^{\prime}\left(\frac{l}{2}\right) \\
W_{1}^{\prime \prime}\left(\frac{l}{2}\right)=W_{2}^{\prime \prime}\left(\frac{l}{2}\right) \\
E I_{y} W_{1}^{\prime \prime \prime}\left(\frac{l}{2}\right)-E I_{y} W_{2}^{\prime \prime \prime}\left(\frac{l}{2}\right)=M \ddot{W}_{1}\left(\frac{l}{2}\right)
\end{gathered}
$$

By applying all these conditions, a matrix-form relation can be written as:

$$
\left[B^{s}\right]\left\{A^{s}\right\}=\{0\}
$$

where $\left[B^{S}\right]$ is the $8 \times 8$ matrix is the static transformation matrix and vector $\left\{A^{s}\right\}$ is

$$
\left\{A^{S}\right\}=\left\{A_{1}^{S}, B_{1}^{S}, C_{1}^{S}, D_{1}^{S}, A_{2}^{S}, B_{2}^{S}, C_{2}^{S}, D_{2}^{S}\right\}^{T}
$$

By forming $\operatorname{Det}\left[B^{S}\right]=0$, which gives us the non-zero solutions of vector $\left\{A^{S}\right\}$, the static eigenvalue $\beta_{s}$ and consequently the critical buckling load $P_{c r}=\bar{K}_{1} \beta_{s}^{2}$ will be obtained for a given geometrical and physical parameters of the system. Since there are 8 conditions with 9 unknowns, it should be noted that a certain value of applied buckling load $P$ is required as an initial condition in order to find the ninth unknown coefficient and the exact post-buckled static shape of the whole system from equation (152). After finding the static buckled shape of the system, the next step is finding the dynamic mode shapes about the buckled case, which is obtained by solving the structurally linearized system.

\subsubsection{Dynamic Analysis}


As shown in the previous chapters, the total dynamic solution is the summation of the buckled static shape and the dynamic motion around it. Therefore, the total deflection response can be written as follows:

$$
W_{i}(x, t)=W_{i}^{s}(x)+V_{i}(x, t), \quad(i=1,2)
$$

Substituting this presumed solution into the equations (146) and (147) and dropping nonlinear, force, damping and voltage terms delivers the structurally linearized dynamic equation around the buckled static equilibrium:

$$
\begin{aligned}
& \ddot{V}_{1}+\bar{K}_{1} V_{1}^{\prime \prime \prime \prime}+\left[\bar{P}-\bar{K}_{2} \int_{0}^{\frac{l}{2}}{W_{1}^{s^{\prime}}}^{2} d x\right] V_{1}^{\prime \prime}-2 \bar{K}_{2} W_{1}^{s^{\prime \prime}} \int_{0}^{\frac{l}{2}} W_{1}^{s^{\prime}} V_{1}^{\prime} d x=0 \\
& \ddot{V}_{2}+\bar{K}_{1} V_{2}^{\prime \prime \prime \prime}+\left[\bar{P}-\bar{K}_{2} \int_{\frac{l}{2}}^{l} W_{2}^{s^{\prime 2}} d x\right] V_{2}^{\prime \prime}-2 \bar{K}_{2} W_{2}^{s^{\prime \prime}} \int_{\frac{l}{2}}^{l} W_{2}^{s^{\prime}} V_{2}^{\prime} d x=0
\end{aligned}
$$

To find the natural frequencies and mode shapes of the linearized system, time-dependent harmonic functions are considered to be applied in the following format:

$$
V_{i}=\psi_{i}(x) e^{j \omega t}, \quad(i=1,2)
$$

Inserting solution (162) into equations (160) and (161) gives the following differential equations:

$$
\begin{aligned}
& \bar{K}_{1} \psi_{1}^{\prime \prime \prime \prime}+\bar{Z}_{1} \psi_{1}^{\prime \prime}-\omega^{2} \psi_{1}=2 \bar{K}_{2} W_{1}^{s^{\prime \prime}} \bar{\gamma}_{1} \\
& \bar{K}_{1} \psi_{2}^{\prime \prime \prime \prime}+\bar{Z}_{2} \psi_{2}^{\prime \prime}-\omega^{2} \psi_{2}=2 \bar{K}_{2} W_{2}^{s^{\prime \prime}} \bar{\gamma}_{2}
\end{aligned}
$$

where 


$$
\begin{array}{ll}
\bar{\gamma}_{1}=\int_{0}^{\frac{l}{2}} W_{1}^{s^{\prime}} \psi_{1}^{\prime} d x ; & \bar{Z}_{1}=\left[\bar{P}-\bar{K}_{2} \int_{0}^{\frac{l}{2}}{W_{1}^{s^{\prime}}}^{2} d x\right] \\
\bar{\gamma}_{2}=\int_{\frac{l}{2}}^{l} W_{2}^{s^{\prime}} \psi_{2}^{\prime} d x ; & \bar{Z}_{2}=\left[\bar{P}-\bar{K}_{2} \int_{\frac{l}{2}}^{l}{W_{2}^{s^{\prime}}}^{2} d x\right]
\end{array}
$$

To solve the equations (163) and (164), the general solutions can be divided into homogenous and non-homogenous parts:

$$
\begin{aligned}
& \psi_{1}=\psi_{1 h}+\psi_{1 p} \\
& \psi_{2}=\psi_{2 h}+\psi_{2 p}
\end{aligned}
$$

To find the homogenous solution, the right side of the differential equations are set to zero. Due to the symmetry of the static shape of the buckled beam, we can write $\bar{z}_{1}=\bar{z}_{2}=\bar{z}$. This assumption leads to the following general solutions for the homogenous part:

$$
\begin{gathered}
\psi_{1 h}(x)=A_{1} \sin \left(\beta_{1} x\right)+B_{1} \cos \left(\beta_{1} x\right)+C_{1} \sinh \left(\beta_{2} x\right)+D_{1} \cosh \left(\beta_{2} x\right), \\
0 \leq x \leq \frac{l}{2} \\
\psi_{2 h}(x)=A_{2} \sin \left(\beta_{1} x\right)+B_{2} \cos \left(\beta_{1} x\right)+C_{2} \sinh \left(\beta_{2} x\right)+D_{2} \cosh \left(\beta_{2} x\right), \\
\frac{l}{2} \leq x \leq l
\end{gathered}
$$

where

$$
\beta_{1,2}=\left( \pm \frac{S_{1}}{2}+\frac{\sqrt{S_{1}^{2}+4 S_{2}}}{2}\right)^{\frac{1}{2}}
$$


Since the right-hand side of equations (163) and (164) are harmonic functions of static eigenvalue $\beta_{s}$, the particular solutions for equations (163) and (164) can be formed as:

$$
\begin{aligned}
& \psi_{1 p}(x)=E_{1} \cos \left(\beta_{s} x\right)+F_{1} \sin \left(\beta_{s} x\right) \\
& \psi_{2 p}(x)=E_{2} \cos \left(\beta_{s} x\right)+F_{2} \sin \left(\beta_{s} x\right)
\end{aligned}
$$

By putting these particular solutions into the differential equations followed by some mathematical simplifications, the coefficients of the particular solutions can be written as functions of the homogenous unknown constants. Finally, applying boundary and matching conditions of the buckled beam delivers the following matrix-form equation:

$$
\left[B^{D}\right]\{A\}=\{0\}
$$

where the $8 \times 8$ matrix $\left[B^{D}\right]$ is the dynamic transformation matrix and vector $\{A\}$ is:

$$
\{A\}=\left\{A_{1}, B_{1}, C_{1}, D_{1}, A_{2}, B_{2}, C_{2}, D_{2}\right\}^{T}
$$

The required conditions for non-zero solutions of the equation (171) gives the characteristic equations from which one may obtain the system natural frequencies and mode shapes.

For solving the nonlinear equations (146) and (147), Galerkin's method is chosen as the discretization approach. The presumed solution form of the discretized partial differential equations is made from the obtained shape functions multiplied by unknown generalized coordinates, which can be written in the following format:

$$
W_{i}(x, t)=W_{i}^{s}(x)+\sum_{i=1}^{N} \psi_{j}^{i}(x) q_{j}^{i}(t), \quad(i=1,2)
$$

where, $\psi^{i}(x),(i=1,2, \ldots, N)$ are the dynamic shape functions of the structurally linearized system. An additional assumption is made in the discretization process with 
respect to the voltage produced across the beam thickness. In equations (146) and (147), the voltage is considered as a function of both time and space. For the described system, it is a valid assumption to consider the voltage only as a function of time. Such consideration followed by an execution of integration by part would lead to the following form of discretized structural equations for each part:

$$
\begin{gathered}
\sum_{j=0}^{N} m_{i j}^{1} \ddot{q}_{1}^{j}+\sum_{j=0}^{N} c_{i j}^{1} \dot{q}_{1}^{j}+\sum_{j=0}^{N} k_{i j}^{1} q_{1}^{j}+\sum_{j=1}^{N} \sum_{k=1}^{N} A_{i j k}^{1} q_{1}^{j} q_{1}^{k}+\sum_{j=0}^{N} \sum_{k=0}^{N} \sum_{l=0}^{N} B_{i j k l}^{1} q_{1}^{j} q_{1}^{k} q_{1}^{l} \\
+\sum_{j=0}^{N} \alpha_{i j}^{21} q_{1}^{j} v(t)+\alpha_{i}^{11} v(t)+f_{1}^{i}=0, \quad(i=0,1,2, \ldots, N) \\
\sum_{j=0}^{N} m_{i j}^{2} \ddot{q}_{2}^{j}+\sum_{j=0}^{N} c_{i j}^{2} \dot{q}_{2}^{j}+\sum_{j=0}^{N} k_{i j}^{2} q_{2}^{j}+\sum_{j=1}^{N} \sum_{k=1}^{N} A_{i j k}^{2} q_{2}^{j} q_{2}^{k}+\sum_{j=0}^{N} \sum_{k=0}^{N} \sum_{l=0}^{N} B_{i j k l}^{2} q_{2}^{j} q_{2}^{k} q_{2}^{l} \\
+\sum_{j=0}^{N} \alpha_{i j}^{22} q_{2}^{j} v(t)+\alpha_{i}^{12} v(t)+f_{2}^{i}=0, \quad(i=0,1,2, \ldots, N)
\end{gathered}
$$

where

$$
\begin{aligned}
& m_{i j}^{1}=\int_{0}^{\frac{l}{2}} \psi_{1}^{i} \psi_{1}^{j} d x, \quad c_{i j}^{1}=\bar{C} m_{i j}^{1}, \quad f_{1}^{i}=\int_{0}^{\frac{l}{2}} \ddot{W}_{B} \psi_{1}^{i} d x \\
& m_{i j}^{2}=\int_{\frac{l}{2}}^{l} \psi_{2}^{i} \psi_{2}^{j} d x, \quad c_{i j}^{2}=\bar{C} m_{i j}^{2}, \quad f_{2}^{i}=\int_{\frac{l}{2}}^{l} \ddot{W}_{B} \psi_{2}^{i} d x
\end{aligned}
$$




$$
\begin{aligned}
& k_{i j}^{1}=\bar{K}_{1} \int_{0}^{\frac{l}{2}} \psi_{1}^{j^{\prime \prime \prime \prime}} \psi_{1}^{i} d x+\left[\bar{P}-\bar{K}_{2} \int_{0}^{\frac{l}{2}}{W_{1}^{S^{\prime}}}^{2} d x\right] \int_{0}^{\frac{l}{2}} \psi_{1}^{j^{\prime \prime}} \psi_{1}^{i} d x \\
& -2 \bar{K}_{2} \int_{0}^{\frac{l}{2}} W_{1}^{s^{\prime \prime}} \psi_{1}^{i} d x \int_{0}^{\frac{l}{2}} W_{1}^{s^{\prime}} \psi_{1}^{j^{\prime}} d x \\
& k_{i j}^{2}=\bar{K}_{1} \int_{\frac{l}{2}}^{l} \psi_{2}^{j^{\prime \prime \prime \prime \prime}} \psi_{2}^{i} d x+\left[\bar{P}-\bar{K}_{2} \int_{\frac{l}{2}}^{l}{W_{2}^{s^{\prime}}}^{2} d x\right] \int_{\frac{l}{2}}^{l} \psi_{2}^{j^{\prime \prime}} \psi_{2}^{i} d x \\
& -2 \bar{K}_{2} \int_{\frac{l}{2}}^{l} W_{2}^{s^{\prime \prime}} \psi_{2}^{i} d x \int_{\frac{l}{2}}^{l} W_{2}^{s^{\prime}} \psi_{2}^{j^{\prime}} d x \\
& A_{i j k}^{1}=-\bar{K}_{2} \int_{0}^{\frac{l}{2}} W_{1}^{s^{\prime \prime}} \psi_{1}^{i} d x \int_{0}^{\frac{l}{2}} \psi_{1}^{j^{\prime}} \psi_{1}^{k^{\prime}} d x-2 \bar{K}_{2} \int_{0}^{\frac{l}{2}} \psi_{1}^{j^{\prime \prime}} \psi_{1}^{i} d x \int_{0}^{\frac{l}{2}} W_{1}^{s^{\prime}} \psi_{1}^{k^{\prime}} d x \\
& A_{i j k}^{2}=-\bar{K}_{2} \int_{\frac{l}{2}}^{l} W_{2}^{s^{\prime \prime}} \psi_{2}^{i} d x \int_{\frac{l}{2}}^{l} \psi_{2}^{j^{\prime}} \psi_{2}^{k^{\prime}} d x-2 \bar{K}_{2} \int_{\frac{l}{2}}^{l} \psi_{2}^{j^{\prime \prime}} \psi_{2}^{i} d x \int_{\frac{l}{2}}^{l} W_{2}^{s^{\prime}} \psi_{2}^{k^{\prime}} d x \\
& B_{i j k l}^{1}=-\bar{K}_{2} \int_{0}^{\frac{l}{2}} \psi_{1}^{j^{\prime \prime}} \psi_{1}^{i} d x \int_{0}^{\frac{l}{2}} \psi_{1}^{k^{\prime}} \psi_{1}^{l^{\prime}} d x \quad, \quad B_{i j k l}^{2}=-\bar{K}_{2} \int_{\frac{l}{2}}^{l} \psi_{2}^{j^{\prime \prime}} \psi_{2}^{i} d x \int_{\frac{l}{2}}^{l} \psi_{2}^{k^{\prime}} \psi_{2}^{l^{\prime}} d x \\
& \alpha_{i}^{11}=-\bar{\alpha}_{1} \int_{0}^{\frac{l}{2}} \psi_{1}^{i^{\prime \prime}} d x-\frac{\bar{\alpha}_{2} l}{2} \int_{0}^{\frac{l}{2}} W_{1}^{s^{\prime \prime}} \psi_{1}^{i} d x, \quad \alpha_{i j}^{21}=-\frac{\bar{\alpha}_{2} l}{2} \int_{0}^{\frac{l}{2}} \psi_{1}^{j^{\prime \prime}} \psi_{1}^{i} d x \\
& \alpha_{i}^{12}=-\bar{\alpha}_{1} \int_{\frac{l}{2}}^{l} \psi_{2}^{i \prime \prime} d x-\frac{\bar{\alpha}_{2} l}{2} \int_{\frac{l}{2}}^{l} W_{2}^{s^{\prime \prime}} \psi_{2}^{i} d x, \quad \alpha_{i j}^{22}=-\frac{\bar{\alpha}_{2} l}{2} \int_{\frac{l}{2}}^{l} \psi_{2}^{j^{\prime \prime}} \psi_{2}^{i} d x
\end{aligned}
$$

Equations (174) and (175) can be written in a matrix format by defining the following generalized coordinates vector for the whole system: 


$$
\begin{gathered}
\underline{q}=\left\{\left\{q_{1}^{1}, q_{1}^{2}, \ldots, q_{1}^{N}\right\}^{T},\left\{q_{2}^{1}, q_{2}^{2}, \ldots, q_{2}^{N}\right\}^{T}\right\}^{T}=\left\{\underline{q}_{1}^{T}, \underline{q}_{2}^{T}\right\}^{T} \\
{[M] \underline{\ddot{q}}+[C] \underline{\dot{q}}+[K] \underline{q}+\underline{f}_{N V}+\left\{\left[\alpha^{2}\right] \underline{q}+\underline{\alpha}^{1}\right\} v(t)=\underline{F}}
\end{gathered}
$$

Equation (177) shows the nonlinear discretized equation for the uncoupled system. Since two parts of the buckled beam are connected in the center by the lumped mass and their responses are coupled via matching conditions, a transformation relation for each considered mode shape can be defined to relate the total generalized coordinates to the generalized coordinates of either part of the beam (in this study first part of the beam is considered as the independent generalized coordinate vector):

$$
\underline{q}=[B] \underline{q}_{1}
$$

Inserting equation (178) into the discretized nonlinear equation (177) followed by premultiplying $[B]^{T}$ delivers the discretized nonlinear equation for the coupled bistable beam as follows:

$$
\left[M_{u}\right] \underline{\ddot{q}}_{1}+\left[C_{u}\right] \underline{\dot{q}}_{1}+\left[K_{u}\right] \underline{q_{1}}+\underline{f}_{N V_{u}}+\left\{\left[\alpha_{u}^{2}\right] \underline{q}+\underline{\alpha}_{u}^{1}\right\} v(t)=\underline{F}_{u}
$$

where

$$
\begin{gathered}
{\left[M_{u}\right]=[B]^{T}[M][B], \quad\left[C_{u}\right]=[B]^{T}[C][B], \quad\left[K_{u}\right]=[B]^{T}[K][B]} \\
{\left[\alpha_{u}^{2}\right]=[B]^{T}\left[\alpha^{2}\right][B], \quad \underline{f}_{N V_{u}}=[B]^{T} \underline{f}_{N V}, \quad \underline{F}_{u}=[B]^{T} \underline{F}, \quad \underline{\alpha}_{u}^{1}=[B]^{T} \underline{\alpha}^{1}}
\end{gathered}
$$

Equation (179) consists of unknown generalized coordinates and the voltage function, and requires the additional electrical equation obtained for the piezoelectric buckled beam (Eq. (145)) to solve. Completing this solution requires assumption of an external electrical load on the system so that a relation between the electrical charge and output voltage can be 
written. For simplicity, a purely resistive load $R$ is considered as the external load of the energy harvester. The equivalent circuit design for such a system with a piezoelectric capacitance is shown in Figure 31. The whole electrical charge can be written as a function of the voltage across the buckled beam as follows:

$$
\dot{Q}(t)=\int_{0}^{l} q(x, t) d x=\frac{v(t)}{R}
$$

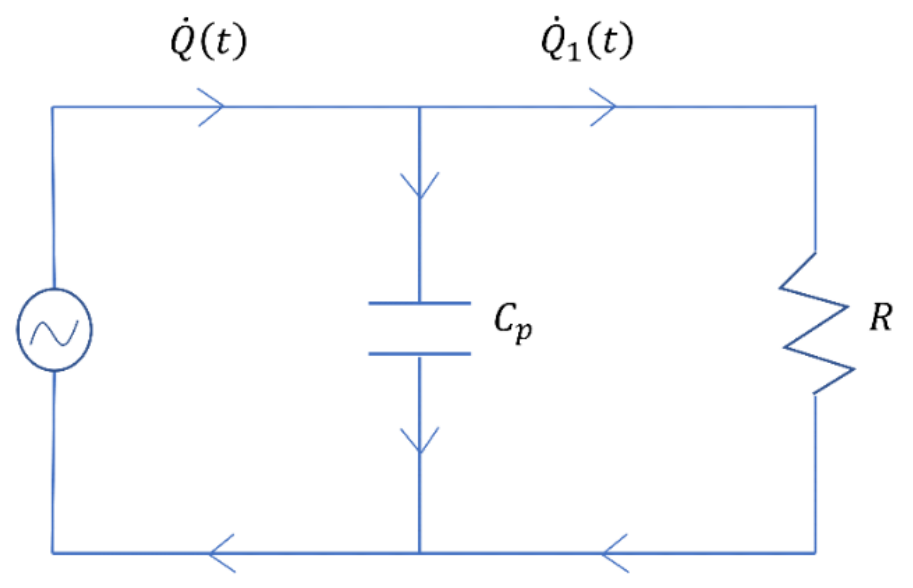

Figure 31. Equivalent circuit design for the bistable vibration energy harvester with an external resistive load.

By inserting relation (180) into equation (145), the following differential equation can be found for the output voltage:

$$
\begin{gathered}
c_{p} \dot{v}(t)+\bar{\beta}_{0} \frac{v(t)}{R}+\bar{\beta}_{1}\left[\int_{0}^{\frac{l}{2}} \dot{W}_{1}^{\prime \prime} d x+\int_{\frac{l}{2}}^{l} \dot{W}_{2}^{\prime \prime} d x\right]-2 \bar{\beta}_{2}\left[\int_{0}^{\frac{l}{2}} \dot{W}_{1}^{\prime} W_{1}^{\prime} d x+\int_{\frac{l}{2}}^{l} \dot{W}_{2}^{\prime} W_{2}^{\prime} d x\right] \\
=0
\end{gathered}
$$

where 


$$
c_{p}=1-\frac{d_{31}^{2} E}{\epsilon_{33}^{T}}, \quad \bar{\beta}_{0}=\frac{h}{b l \epsilon_{33}^{T}}, \quad \bar{\beta}_{1}=\frac{h^{2} d_{31} E}{2 \epsilon_{33}^{T} l}, \quad \bar{\beta}_{2}=\frac{h d_{31} E}{2 \epsilon_{33}^{T} l}
$$

Substituting the response for the nonlinear deflection equation (173) into equation (181) yields the final discretized coupled equations for the bistable piezoelectric energy harvester:

$$
\begin{gathered}
{\left[M_{u}\right] \underline{\ddot{q}_{1}}+\left[C_{u}\right] \underline{\dot{q}_{1}}+\left[K_{u}\right] \underline{q_{1}}+\underline{f}_{N V_{u}}+\left\{\left[\alpha_{u}^{2}\right] \underline{q}+\underline{\alpha}_{u}^{1}\right\} v(t)=\underline{F}_{u}} \\
c_{p} \dot{v}(t)+\bar{\beta}_{0} \frac{v(t)}{R}+\sum_{i=0}^{N} \beta_{i}^{11} \dot{q}_{1}^{i}+\sum_{i=0}^{N} \beta_{i}^{12} \dot{q}_{2}^{i}+\sum_{i=0}^{N} \sum_{j=0}^{N} \beta_{i j}^{21} q_{1}^{i} \dot{q}_{1}^{j}+\sum_{i=0}^{N} \sum_{j=0}^{N} \beta_{i j}^{22} q_{2}^{i} \dot{q}_{2}^{j} \\
=0
\end{gathered}
$$

where

$$
\begin{aligned}
& \beta_{i}^{11}=\bar{\beta}_{1} \int_{0}^{\frac{l}{2}} \psi_{1}^{i^{\prime \prime}} d x-2 \bar{\beta}_{2} \int_{0}^{\frac{l}{2}} W_{1}^{s^{\prime}} \psi_{1}^{j^{\prime}} d x, \quad \beta_{i j}^{21}=-2 \bar{\beta}_{2} \int_{0}^{\frac{l}{2}} \psi_{1}^{i^{\prime}} \psi_{1}^{j^{\prime}} d x \\
& \beta_{i}^{12}=\bar{\beta}_{1} \int_{\frac{l}{2}}^{l} \psi_{2}^{i^{\prime \prime}} d x-2 \bar{\beta}_{2} \int_{\frac{l}{2}}^{l} W_{2}^{s^{\prime}} \psi_{2}^{j^{\prime}} d x, \quad \beta_{i j}^{22}=-2 \bar{\beta}_{2} \int_{\frac{l}{2}}^{l} \psi_{2}^{i^{\prime}} \psi_{2}^{j^{\prime}} d x
\end{aligned}
$$

Solving the coupled equations (182) and (183) numerically delivers the nonlinear deflection and output voltage of the described piezoelectric buckled system under base excitation. This is the critical relationship needed to predict power production for this simplistic nonlinear energy harvester.

\subsection{Experimental Setup}

To verify the proposed modeling approach, specimens for experimental testing based on sheets of commercial polarized PVDF sandwiched between two thin copper layer produced 
by Airmar Piezoflex PVDF were constructed (Figure 32). As shown in the figure, the beam was buckled by applying compressive end-condition displacement to one end while keeping the other end fixed. The buckled energy harvester system was rigidly fixed on a custom-built shaker table. A laser displacement sensor (optoNCDT 1401) was used to measure the vertical (transverse) motion of the central mass and the output voltage was measured using Agilent DSO9104A Digital Storage Oscilloscope with $1 \mathrm{GHz}$ bandwidth capacity.

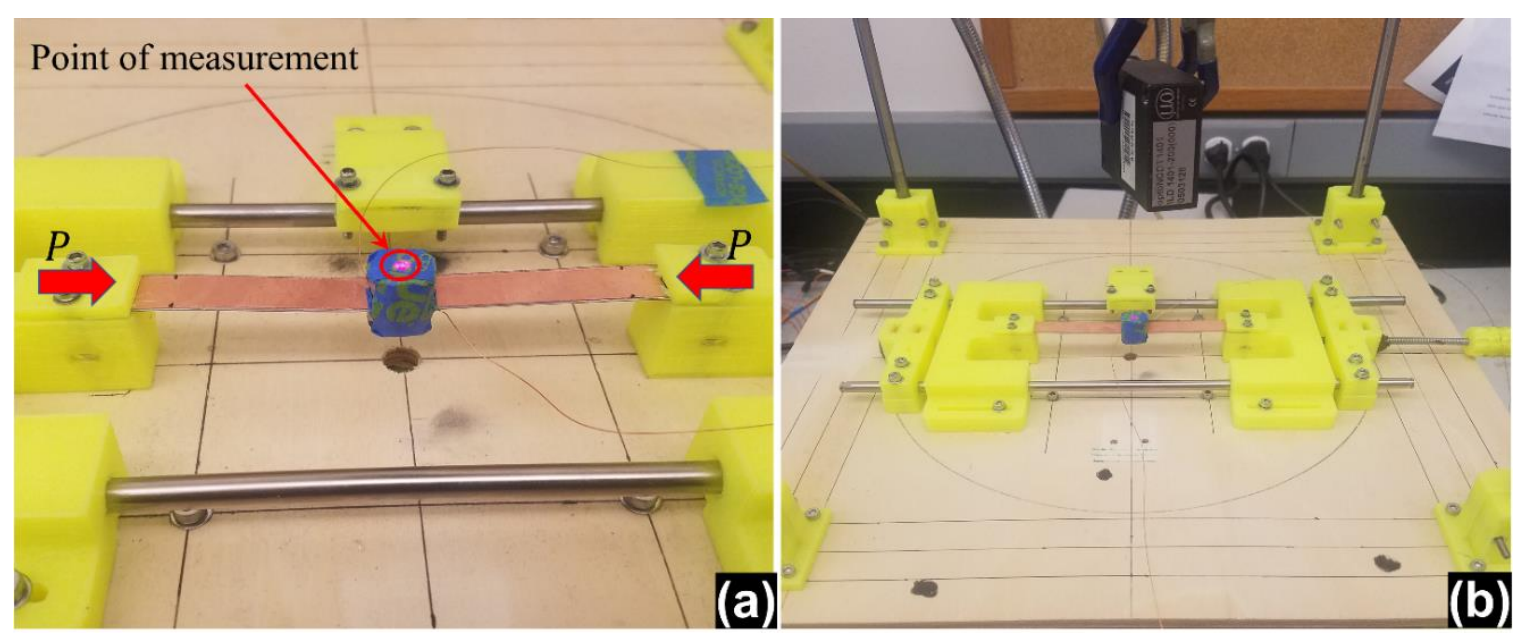

Figure 32. a) Buckled beam sample made of commercial PVDF with a central mass, b) the whole experimental setup of the shaker table and the laser sensor.

Instead of assuming literature material values, a number of mechanical parameters were measured experimentally to improve accuracy of the developed model. Since the sample beam is a composite of three layers, a thick PVDF layer with two thin copper layers at its top and bottom, the equivalent Young's modulus of the sample must be measured due to the large difference between the elastic stiffness of PVDF and copper. Conventional tensile testing produces questionable Young's modulus values for the composite beams due influences of the specimen gripping causing premature failure of the thin outer copper 
layers, resulting in pulling only a relatively pure PVDF sample rather than capturing the stiffness of the entire composite beam. To avoid this issue, a simple static bending test was designed as an alternative method to measure Young's modulus. As schematically shown in Figure 33, a cantilever beam sample of the commercial PVDF sheet was selected and the lateral deflection due to a small weight applied to the tip of the beam was measured by the laser sensor. Using linear static beam theory, the equivalent Young's modulus of the tested sample was calculated $4 G P a$.
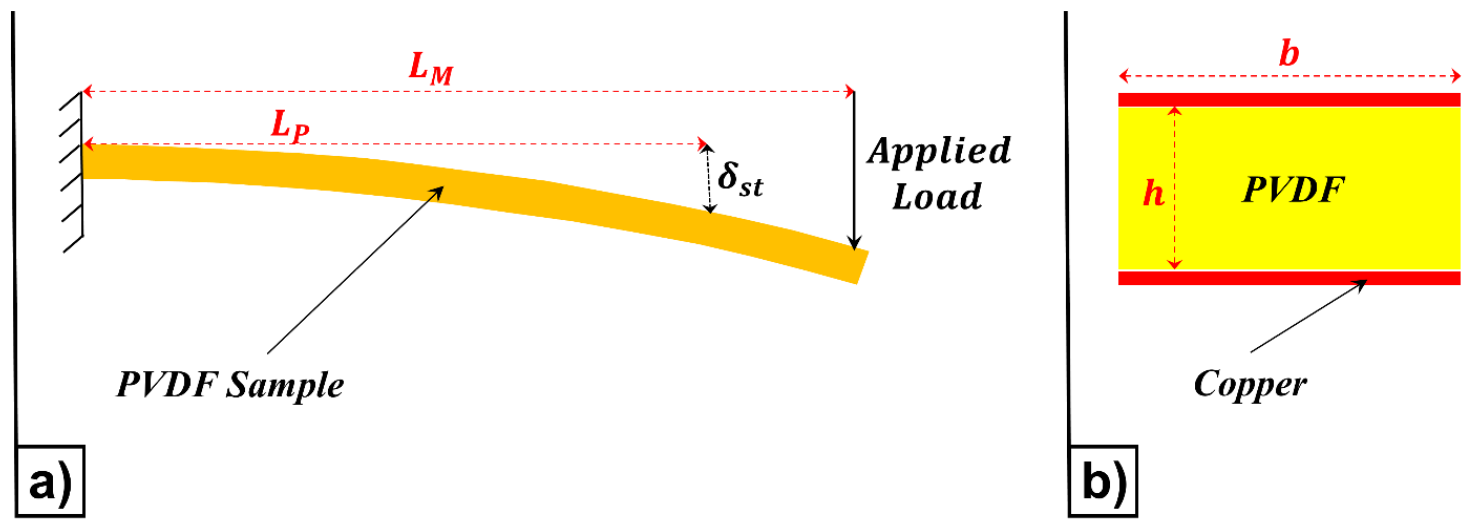

Figure 33. Schematic view of static bending test on a cantilever sample of the commercial PVDF sheet: a) side view, b) cross-sectional view.

The structural damping coefficient and the equivalent external resistive load of the experimental setup was also measured by performing a free vibration test. In this test, a cantilever beam sample was slightly plucked into its first vibrational mode as shown in Figure 34 and then released in a way that the lateral deflection and output voltage response of the free vibrational motion were measured experimentally. The amplitude response was fitted into a single degree of freedom vibrational system from which the damping coefficient and the structural damping of the beam were estimated. By assuming the piezoelectric coefficient for the PVDF sample (obtained from Airmar Piezoflex datasheet), 
the voltage response can be also calculated for a particular resistive load for this single degree of freedom system. By comparing this evaluated response with the one measured from the experimental test, the assumed electrical resistance can be adjusted so that these two responses are matched. The difference between the presumed and actual resistive load is due to several factors including the mechanical and electrical connections, material properties imperfection etc. This can be observed in Figure 35, where two responses (experimental and estimated model) are plotted for a resistive load of $0.8 \mathrm{M} \Omega$, which was found to be the optimal load to give equivalent responses for the materials used in this work.

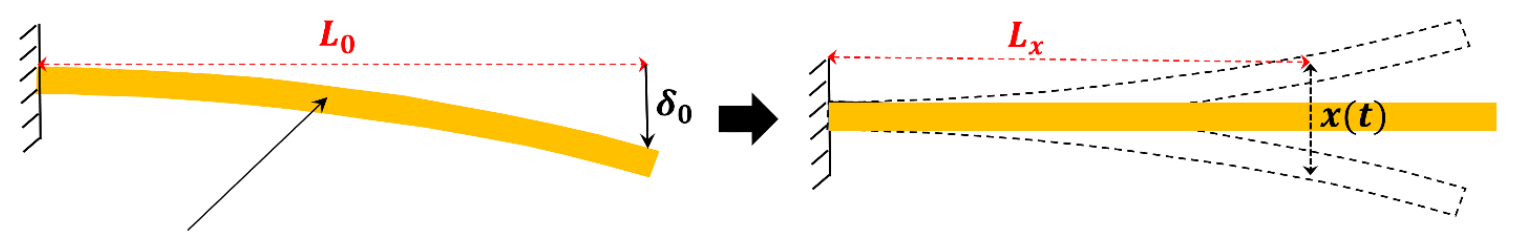

PVDF Sample

Figure 34. Schematic view of the free vibration test on a cantilever sample of the commercial PVDF sheet.

For the buckled beam device structure, the assumed resistive load and the experimentally measured parameters are listed in Table 4. In the next section, dynamic behavior and output voltage response of the bistable device under various driving conditions measured from experimental tests, including some regimes of buckled state switching, are evaluated to provide model validation.

Table 4. Experimental sample dimensions and mechanical/electrical properties. 


\begin{tabular}{|l|c|c|c|c|c|c|c|c|}
\hline \multirow{2}{*}{ Parameters } & $\begin{array}{c}\text { Beam } \\
\text { length } \\
(\mathrm{mm})\end{array}$ & $\begin{array}{c}\text { Beam } \\
\text { width } \\
(\mathrm{mm})\end{array}$ & $\begin{array}{c}\text { Beam } \\
\text { thickness } \\
(\mathrm{mm})\end{array}$ & $\begin{array}{c}\text { Tip } \\
\text { mass } \\
(\mathrm{gr})\end{array}$ & $\begin{array}{c}\text { Young's } \\
\text { Modulus } \\
(G P a)\end{array}$ & $\begin{array}{c}\text { Structural } \\
\text { damping } \\
(\mathrm{N} . \mathrm{s} / \mathrm{m})\end{array}$ & $\begin{array}{c}d_{31} \\
\text { coefficient } \\
(p C / N)\end{array}$ & $\begin{array}{c}\text { Resistive } \\
\text { load } \\
(M \Omega)\end{array}$ \\
\hline Values & 110 & 10.00 & 0.60 & 8.15 & 4.00 & 0.10 & 14 & 0.80 \\
\hline
\end{tabular}

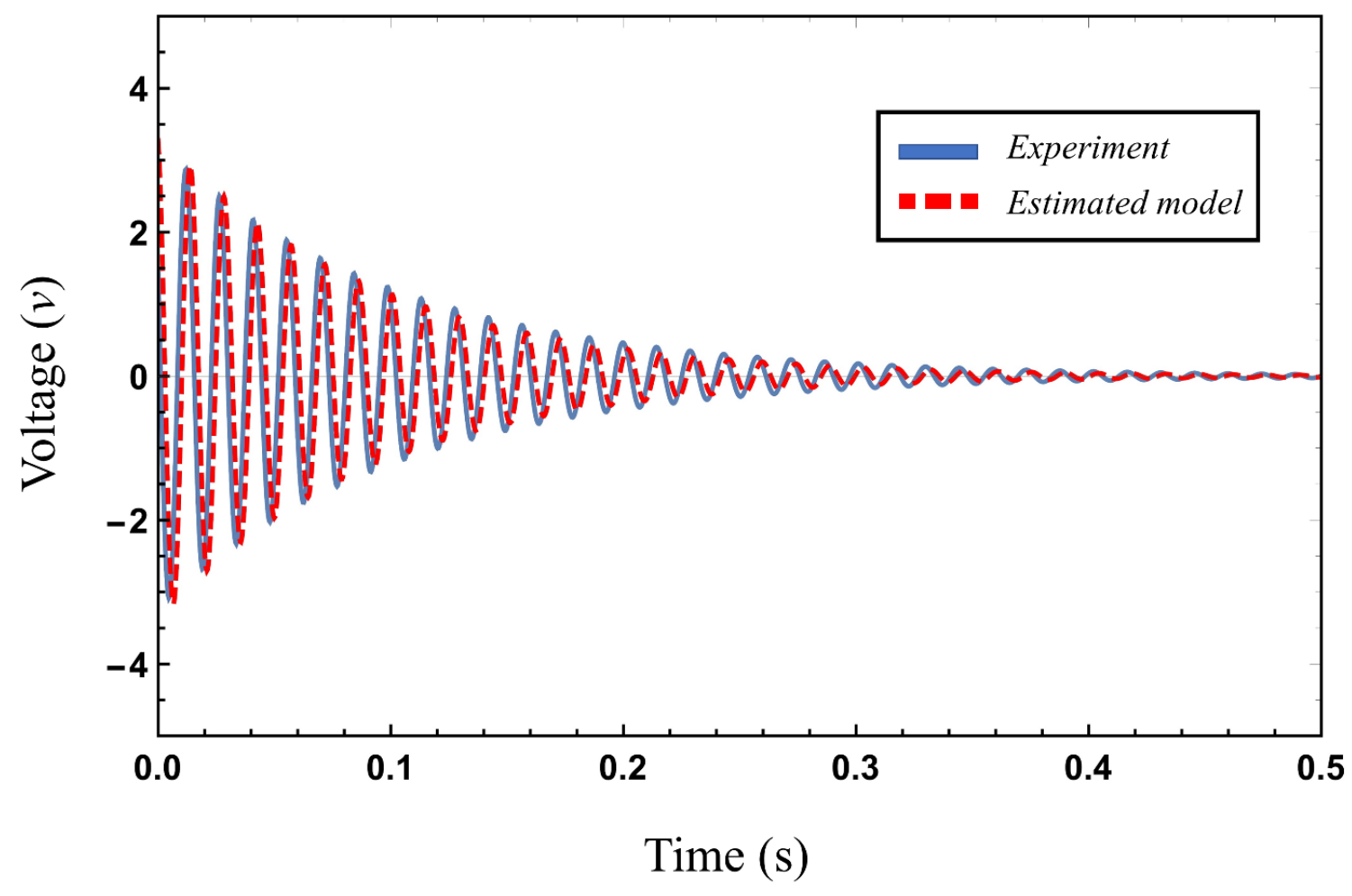

Figure 35. Output voltage response of the free vibration test on the commercial PVDF cantilever sample for $R=0.8 \mathrm{M} \Omega$.

\subsection{Results and Discussion}

In this section, first, the modeling results of the nonlinear static response and first natural frequency of the linearized buckled system are compared with the experimental measurements. For the static buckled configuration of the experimental system, the static deflection of the central mass $\left(\delta_{s t}\right)$ and its corresponding sliding displacement of the 
movable ending fixture $\left(\Delta_{s t}\right)$ were measured (Figure 36). To induce the same buckling deflection $\delta_{s t}$ (measured at $1.5 \mathrm{~mm}$ here) for the modeled system, a $12 \mathrm{~N}$ post-buckling load is required to apply to the system (note that the first critical buckling load calculated in the model is $2.21 \mathrm{~N}$ ). Applying this same buckling load in the model system and calculating $\Delta_{s t}$ from equation (184) based on the nonlinear Euler-Bernoulli beam theory, it was observed that the modeling value $\left(\Delta_{s t-\text { model }}=0.048 \mathrm{~mm}\right)$ matched very well with the one measured from the experiment $\left(\Delta_{s t-\exp }=0.050 \mathrm{~mm}\right)$.

$$
\Delta_{s t}=\frac{1}{2}\left(\int_{0}^{l / 2}{W_{1}^{s^{\prime}}}^{2} d x+\int_{l / 2}^{l}{W_{2}^{s^{\prime}}}^{2} d x\right)
$$

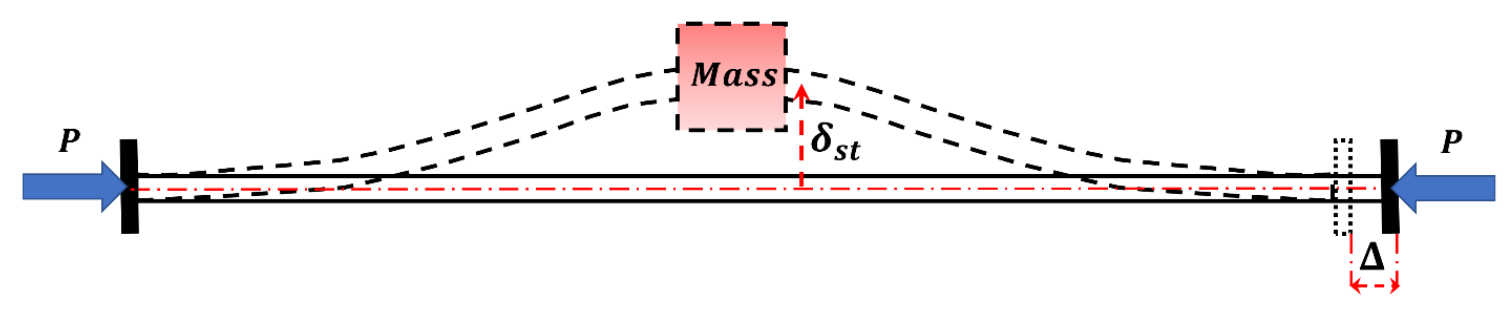

Figure 36. Schematic view of the static deflection and its corresponding displacement of the movable ending fixture.

To measure the first natural frequency of the system, a free vibration test was performed on the buckled device, similar to that performed on the cantilever sample. It should be noted that conventional impact test using accelerometers to find the natural frequencies and mode shapes of the system was not possible due to the relatively small size scale of the tested sample. The first natural frequency measured from experimental testing $(47.23 \mathrm{~Hz})$ agrees very well with the one calculated from the model $(47.69 \mathrm{~Hz})$. These experimental results appear to validate the proposed model system in the post-buckled static and 
linearized dynamic response. Table 5 shows the critical buckling load and the first three natural frequencies obtained from the model for the tested sample. Validation of higher natural frequencies requires a shaker table with a greater driving frequency range limitation.

Table 5. Critical buckling load and first three natural frequencies obtained for the experimental sample.

\begin{tabular}{|l|c|c|c|c|}
\hline & $\begin{array}{c}\text { Critical buckling } \\
\text { load }(N)\end{array}$ & $1^{\text {st }}$ mode $(\mathrm{Hz})$ & $2^{\text {nd }}$ mode $(\mathrm{Hz})$ & $3^{\text {rd }}$ mode $(\mathrm{Hz})$ \\
\hline Model results & 2.21 & 47.69 & 166.12 & 265.87 \\
\hline
\end{tabular}

After verifying the nonlinear static and linearized dynamic response of the system, the nonlinear dynamic behavior under harmonic base excitation was then analyzed. Three mode shapes were considered for each component in the nonlinear analysis. The time response of the lateral deflection and its corresponding Poincare plot, along with the output voltage of the device, were both analytically and experimentally probed for three different cases. Figures 37 and 38 show the vibration deflection of the central mass and the output voltage of the system for the excitation amplitude of $0.6 \mathrm{~mm}$ and the frequency of $18 \mathrm{~Hz}$. As can be seen in Figure 37, there is a local vibration around one of the stable states at this driving condition for both experimental and modeling results. The small energy orbit contour around the static point in the Poincare plot also proves the existence of the local vibration for this case. Figure 38 shows both the experimental and modeling output voltage responses for the same case. As expected, the output voltage amplitude is not very large for the local vibration scenario due to the small strain changes induced into the PVDF beam for this type of motion. 

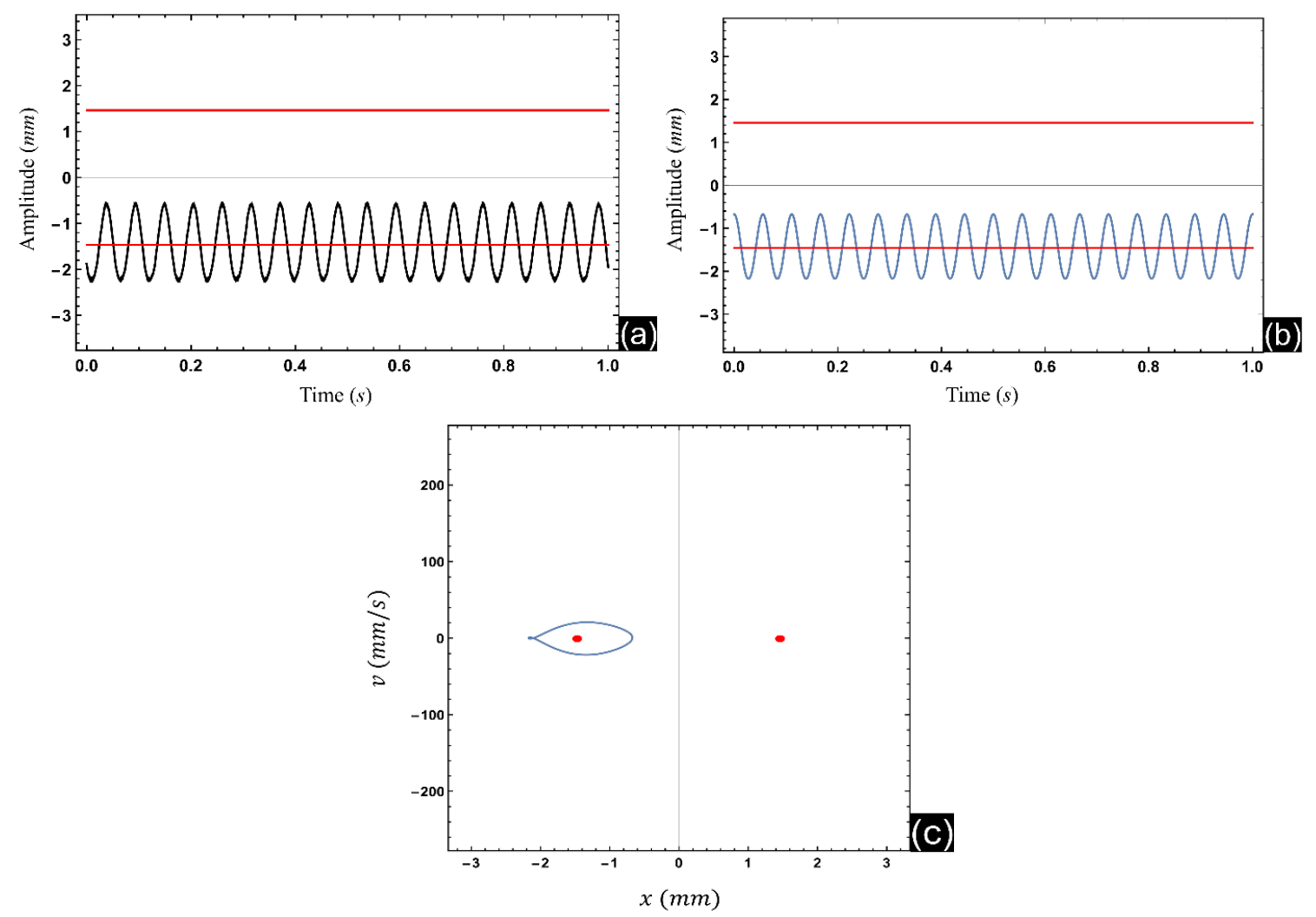

Figure 37. Vibrational amplitude response of the central mass for base amplitude of $0.6 \mathrm{~mm}$ and frequency of $18 \mathrm{~Hz}$ : (a) experimental result; (b) modeling result; (c) Poincare plot.
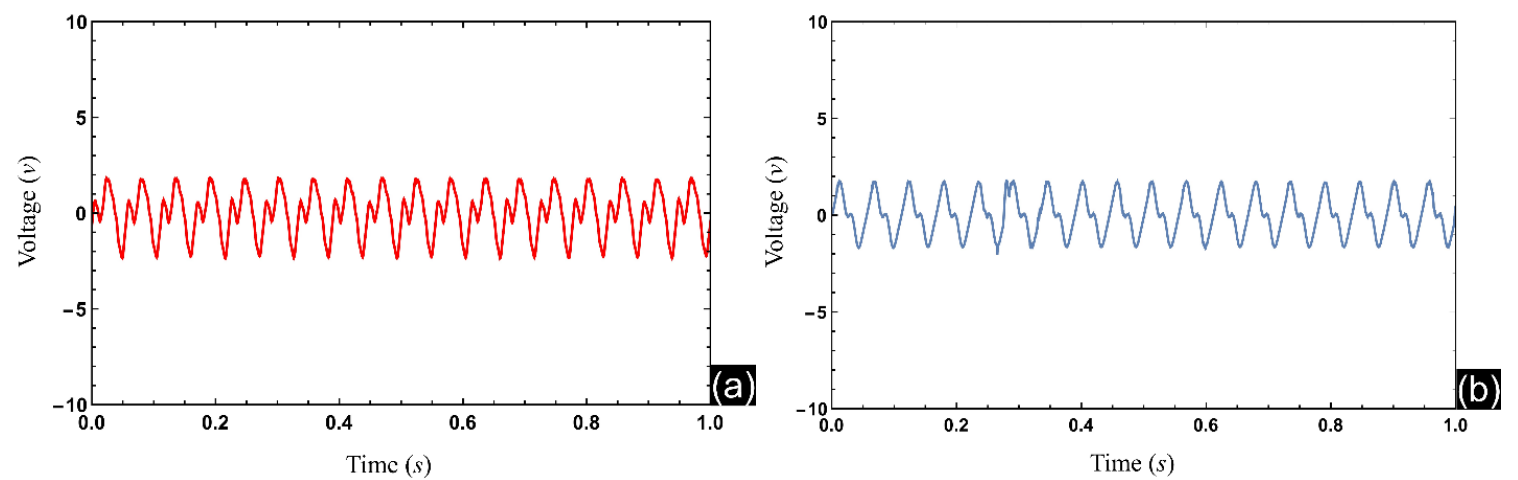

Figure 38. Voltage response of the bistable sample for base amplitude of $0.6 \mathrm{~mm}$ and frequency of $18 \mathrm{~Hz}$ : (a) experimental result; (b) modeling result. 
The next case considered here is the amplitude and voltage response of the experimental bistable sample for base excitation of $0.75 \mathrm{~mm}$ and driving frequency of $24 \mathrm{~Hz}$ (Figures 39 and 40). As can be viewed in Figure 39, a snap-through motion was observed for both experimental and modeling results in this case, in a way that the central mass moves between the two stable states. This bistable motion can also be verified from the Poincare plot (Figure 39-c), where there is a large, smooth energy contour around two stable static points. As a result of this bistable motion, there is more mechanical strain induced in the PVDF beam and consequently, larger output voltage produced by the energy harvester. Figure 40 shows this larger voltage response, demonstrating the advantage of having snapthrough motion in bistable structures for low-frequency energy harvesting applications.
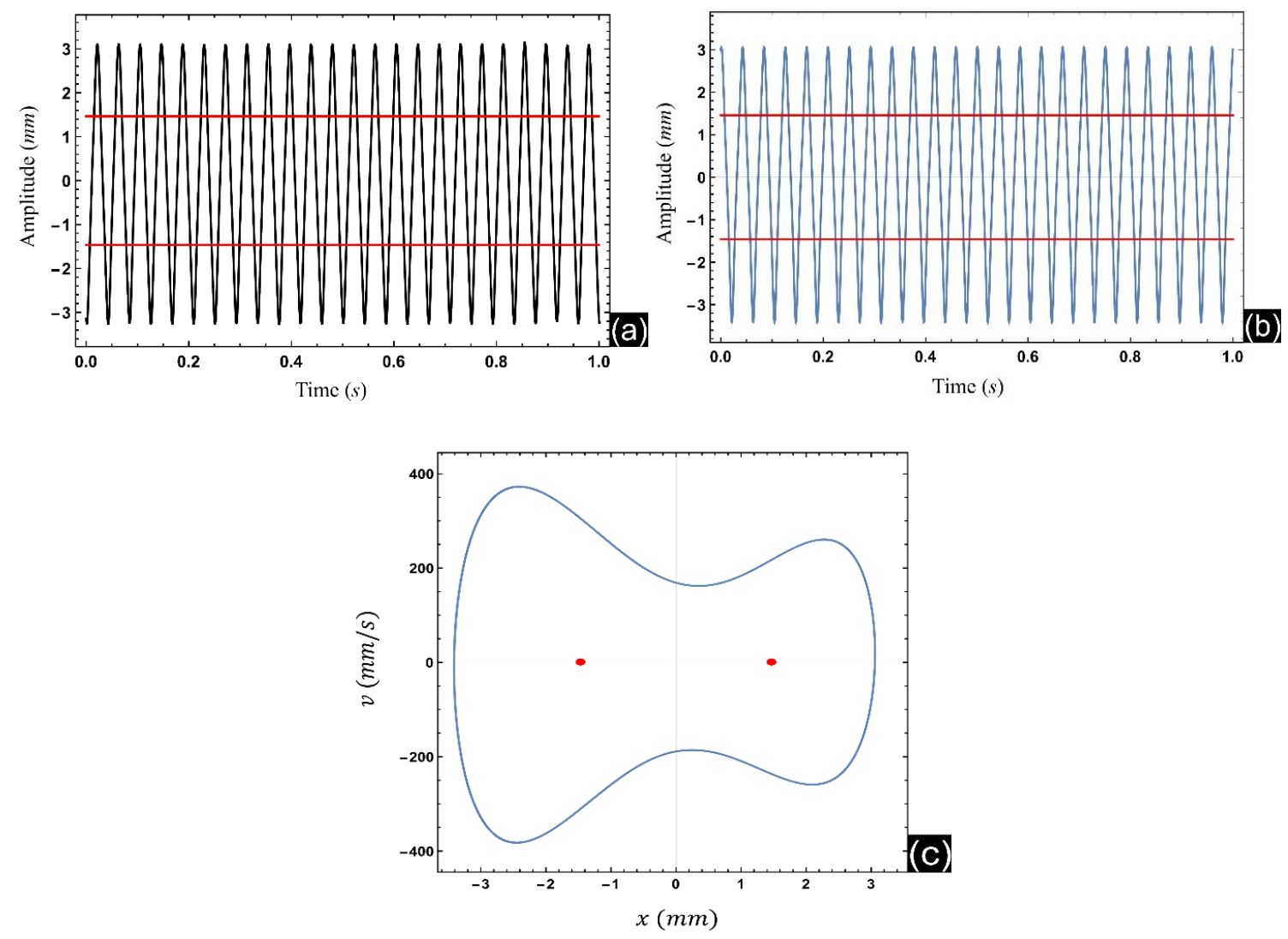
Figure 39. Vibrational amplitude response of the central mass for base amplitude of $0.75 \mathrm{~mm}$ and frequency of $24 \mathrm{~Hz}$ : (a) experimental result; (b) modeling result; (c) Poincare plot.
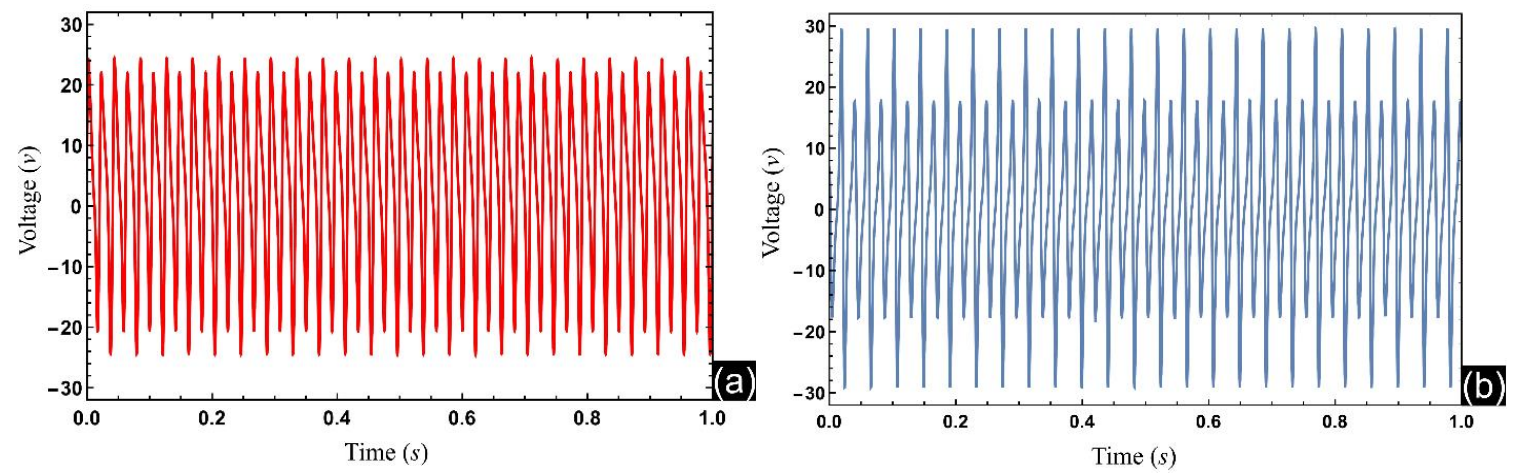

Figure 40. Voltage response of the bistable sample for base amplitude of $0.75 \mathrm{~mm}$ and frequency of $24 \mathrm{~Hz}$ : (a) experimental result; (b) modeling result.

The third case reviewed in this section is the vibration and voltage response of the experimental setup for lower base amplitude and higher driving frequency. For this driving, the base excitation was set to $0.45 \mathrm{~mm}$ at the frequency of $30 \mathrm{~Hz}$. The amplitude response of the central mass, Figure 41, shows that the system experimentally experiences a chaotic motion between its stable states (Figure 41-a) that was also predicted in the model (Figure 41-b). This shows that the presented approach can successfully estimate the chaotic motion if a sufficient number of modes are considered in the nonlinear model, and effectively predict regimes of behavior. This snap-through chaotic motion can be also observed in the Poincare plot developed from the model (Figure 41-c). The voltage response of the experimental system is shown in Figure 42 for the same driving conditions. As can be seen in both experimental and modeling results, there are relatively large variations in the output voltage response of the bistable system which are due to the existence of chaotic motion in 
the buckled beam, with both responses temporarily spiking during a snap-through event. While the stability state switching does not occur in a harmonic manner in this case, the amplitude of voltage response still shows the superiority of bistable structures when it comes to the operational frequency bandwidth.

Figures 43 and 44 show the amplitude-frequency and voltage-frequency response of the studied bistable energy harvester respectively. Due to the shaker table limitations, the frequency range was only considered between 10 to $30 \mathrm{~Hz}$. As can be seen from the figures, there are two peaks in the experimental results for both plots at around one-third and half of the first natural frequency of the system. The existence of these peaks is attributed to super-harmonic resonances observed in the experimental test, which also appeared in the modeling results. It is also shown that the half secondary resonance is more intensified in the model compared to the one-third one. It needs to be mentioned that the amplitude of these peaks in the model and experiment should not match perfectly as a result of modeling simplifications and experimental setup imperfections. However, the existence of these secondary resonances was successfully predicted in the model, which behavior is essential for designing bistable systems suitable for low-frequency energy harvesting applications. In other words, these super-harmonic resonances widen the bistable system operating frequency bandwidth, which results in having snap-through motion within a broader lowfrequency range and consequently lead to a more efficient energy harvester being compatible with real-life applications. 

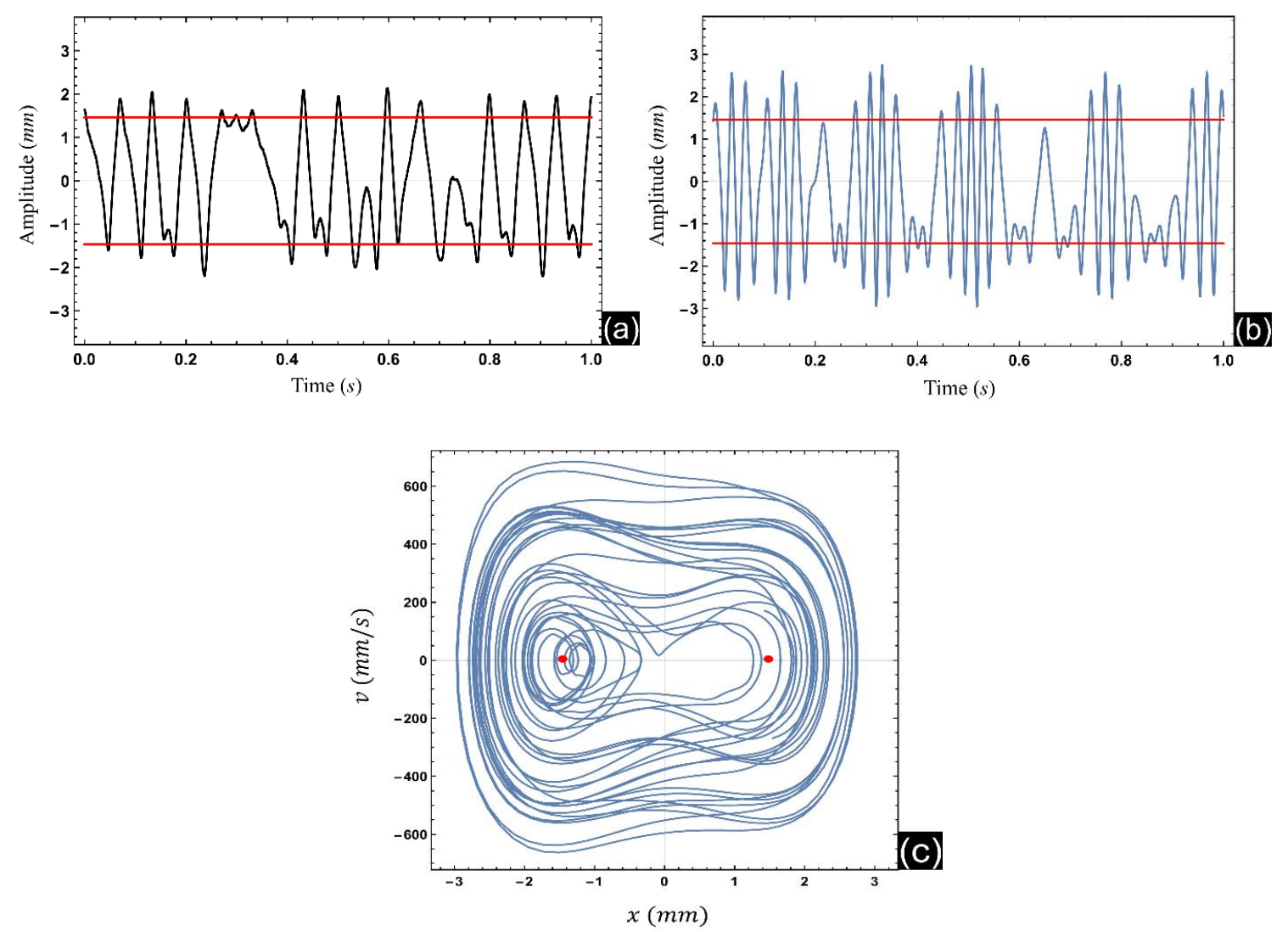

Figure 41. Vibrational amplitude response of the central mass for base amplitude of $0.45 \mathrm{~mm}$ and frequency of $30 \mathrm{~Hz}$ : (a) experimental result; (b) modeling result; (c) Poincare plot.
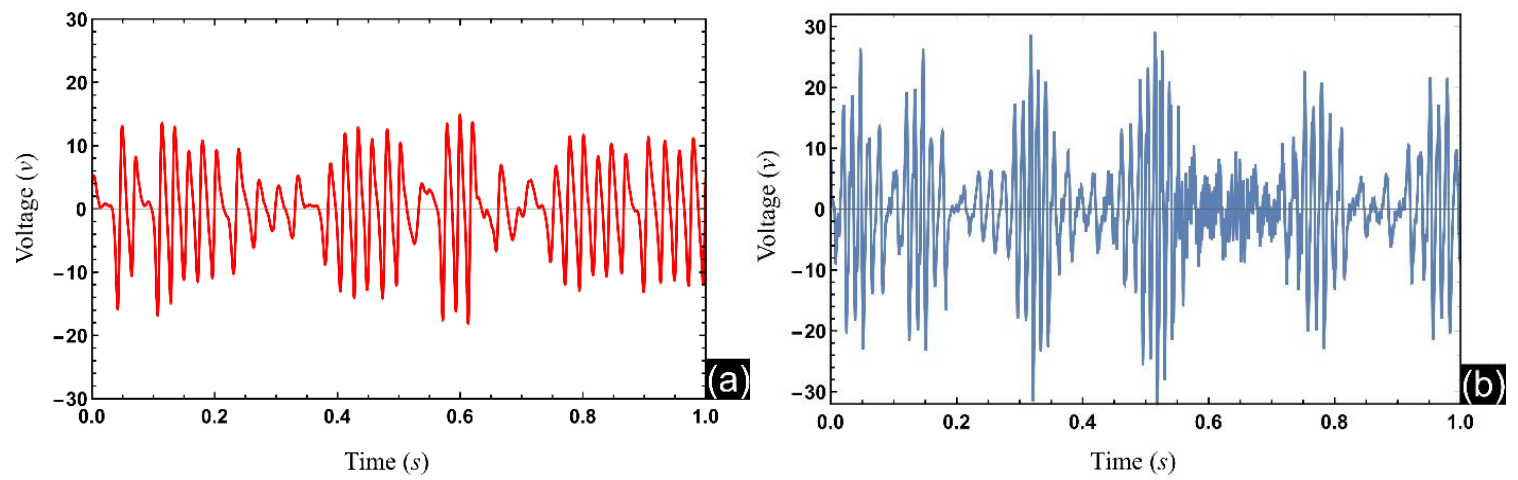

Figure 42. Voltage response of the bistable sample for base amplitude of $0.45 \mathrm{~mm}$ and frequency of $30 \mathrm{~Hz}$ : (a) experimental result; (b) modeling result. 


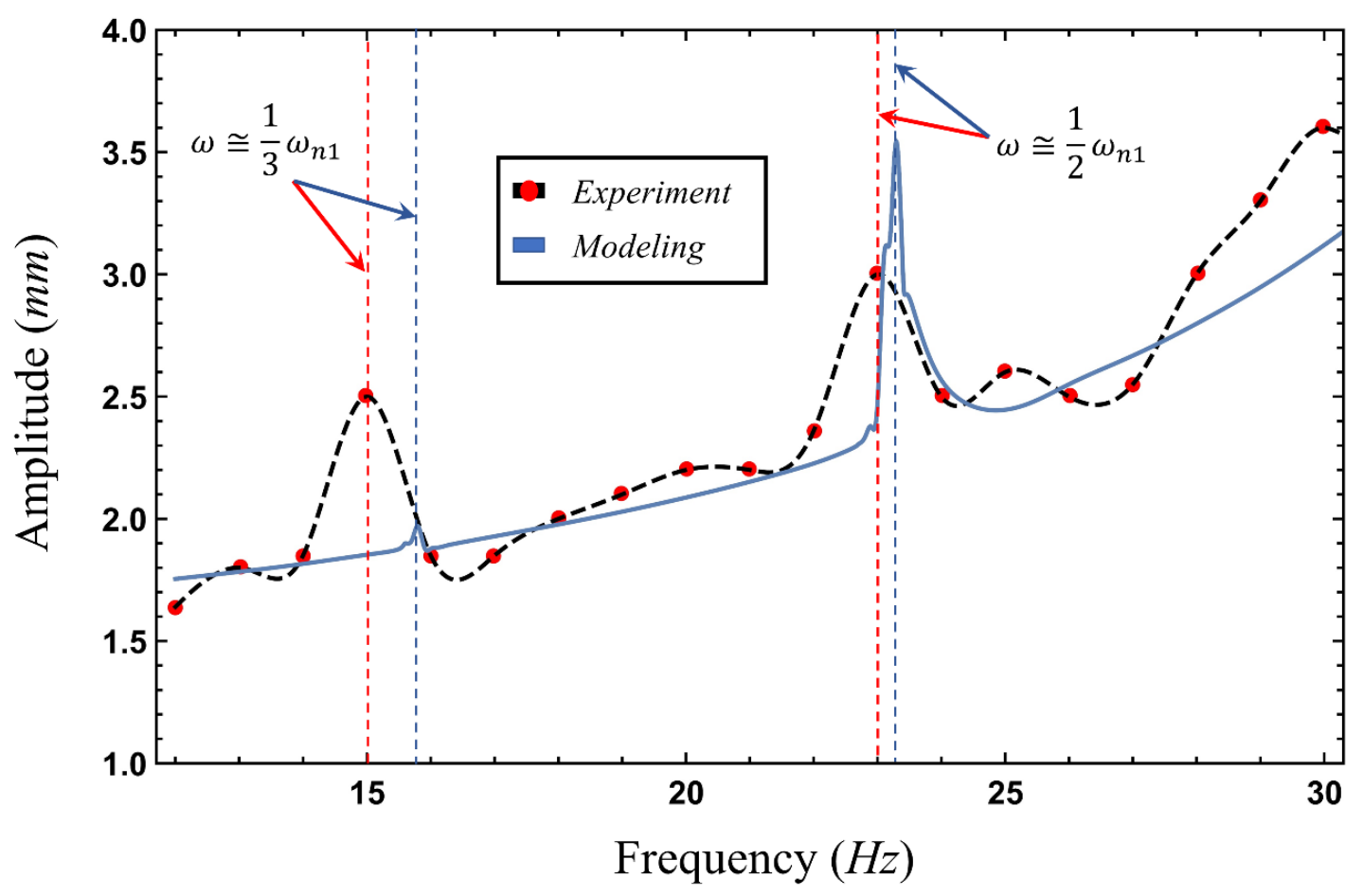

Figure 43. Amplitude-frequency response of the studied experimental bistable sample.

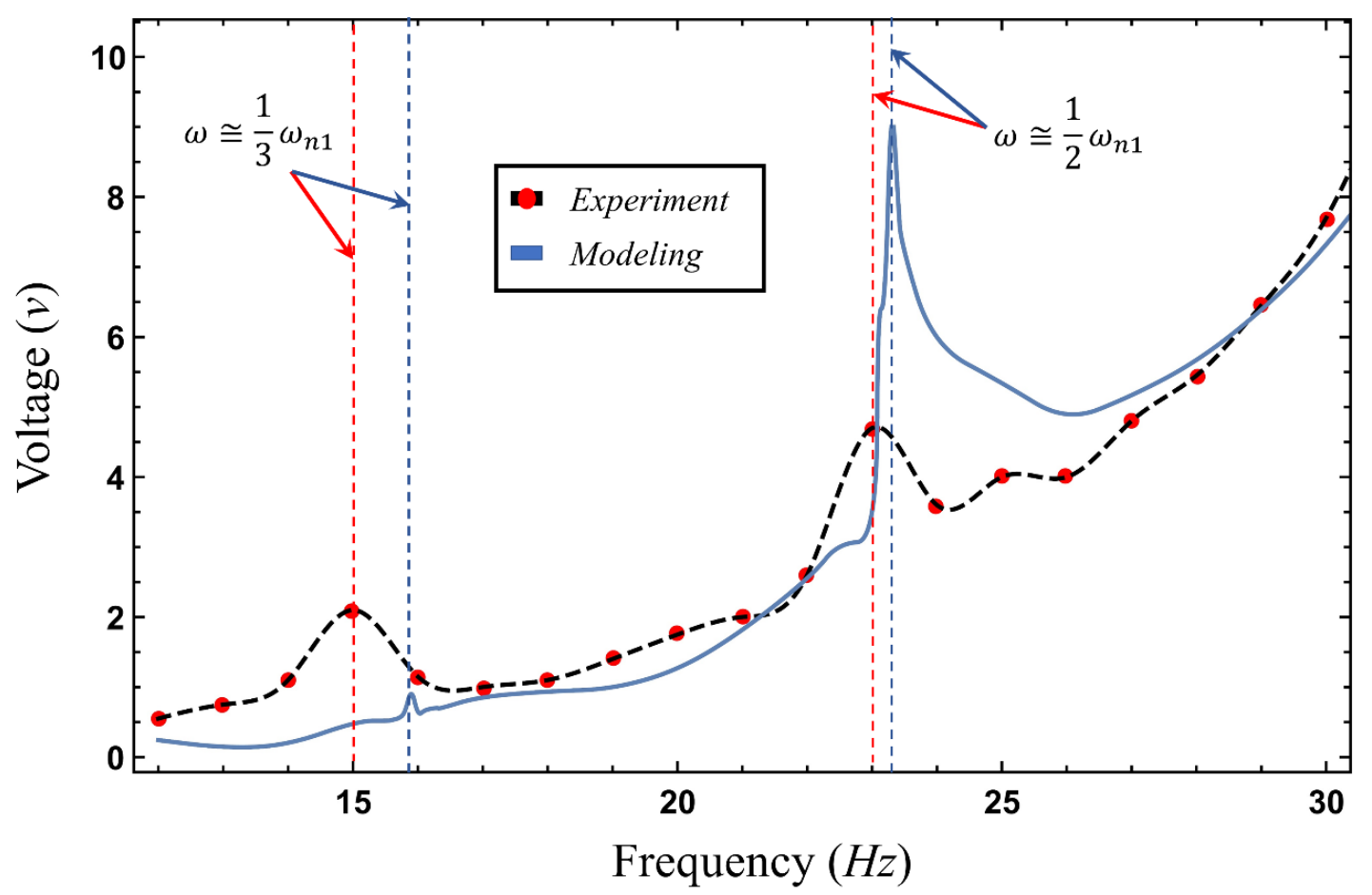

Figure 44. Voltage-frequency response of the studied experimental bistable sample. 
Various results are developed for static, linearized dynamics and nonlinear response of the system in this chapter to prove the accuracy of the proposed coupling approach and its potential for predicting the system nonlinear behavior which is critical for designing purpose. It is also shown that using piezoelectric polymers can be useful in bistable energy harvesters due to the high flexibility they bring to the system at a sacrifice of dropping the electromechanical coupling factor. Furthermore, taking advantage of secondary resonances observed in the experimental results, which are predicted by the model as well, would lead to the broadband operating frequency of the studied device at low-frequency ranges and could be leveraged in designing more complex, multi-component piezoelectric energy harvesters to broaden the viable operating frequency range. 


\section{MICROFABRICATION OF THE DESIGNED MEMS BISTABLE ENERGY HARVESTER}

As discussed in the previous chapters, one of the main issues associated with the current energy harvesters is their incompatibility with the environmental vibrational sources, where more randomly low-frequency oscillations exist. This phenomenon becomes exacerbated for MEMS applications, where the reductions in structure dimensions significantly increases the operational frequency and consequently leads to a more intensified discrepancy between the existing energy harvesters ideal operational frequency range and real-life energy sources. Therefore, one of the primary advantages of this proposed design is its possible utilization in micro-electromechanical systems (MEMS). Chapter five gave a theoretical analysis of the performance of a micro-scale version of the device under various dynamic conditions. However, making an actual MEMS device presents many challenges with the micro-fabrication and characterization processes. In this chapter, we thoroughly investigate the micro-fabrication process steps of the studied energy harvester and try to address sources of device failure prior to completion. In the end, the optimized steps required for building this device are summarized based on the available experimental facilities and some microscopic pictures of a successfully fabricated MEMS device are shown.

\subsection{Process Description}


Microfabrication is a set of standard processes including but not limited to masks design, photolithography, deposition, surface and bulk etching, and characterization required to make miniature structures which are not possible to be built with the conventional manufacturing methods. Each step requires a certain level of knowledge and skills to be performed properly. All the training and fabrication processes in this study have been performed at the University of Louisville Micro/Nano Technology Center [84]. Figure 45 shows a schematic view of the required steps to build a micro-scale version of the studied device. As can be seen in this figure, it is made of four layers in total: structural, bottom electrode, piezoelectric, and top electrode layers, and each step shows a schematic process of the deposition and patterning of each layer in the device. All these layers are designed to form the structure of the device and they were fabricated on the topside of an oxidized wafer.

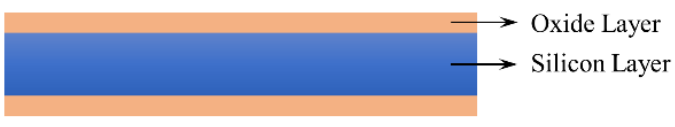

1)

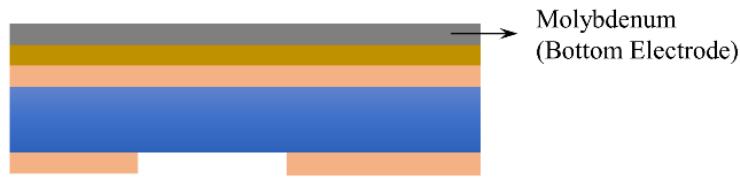

3)

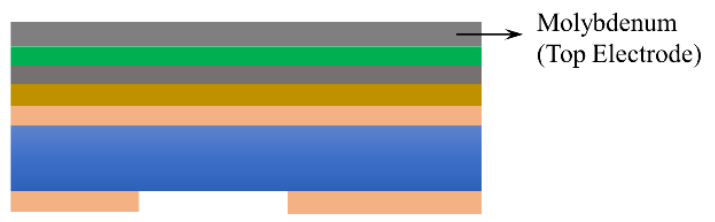

5)

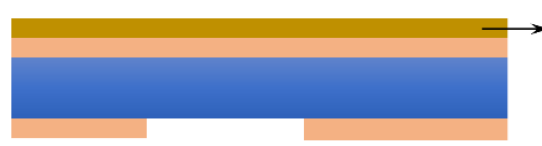

2)

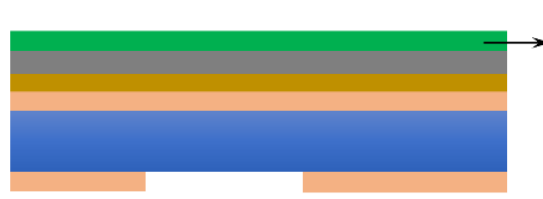

4)

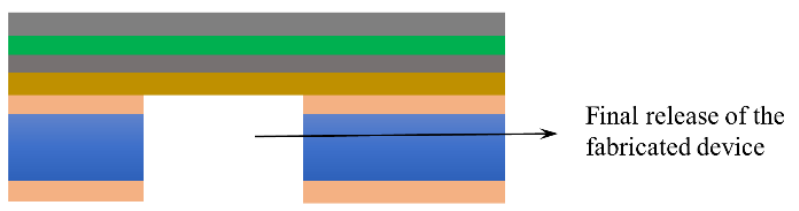

6)

Figure 45. Schematic view of the required steps for micro fabrication process of the MEMS-scale bistable energy harvester.

\subsection{Masks Design}


As explained in the first section of this chapter, microfabrication process includes several depositing, patterning, and etching steps. To pattern each deposited layer, photolithography must be performed, in which a specific type of photoresist is deposited, aligned, exposed, and developed on a silicon wafer. The purpose of this process is to cover and protect certain areas of the deposited layer, so the rest can be etched away from the silicon wafer. In this process, the deposited layer is covered with a chemical photoresist which is sensitive to light and can be developed when it is exposed. Since this exposure is performed by UV light, it requires some photomask or optical mask to protect parts of the wafer where the deposited layer is supposed to be intact.

The fabrication of the proposed device requires five photomasks corresponding to patterning of the following material layers or processes: pre-stressed structure, bottom electrode, piezoelectric, top electrode, and the final bulk etching step. These mask layouts for a single device are displayed in Figures 46-50 separately and Figure 51 superimposed on top of one another. Figure 46 shows the mask designed for the structural layer of the device. Since this is a bistable device, it requires some compressive stress to make the bistability occur in the system. For this purpose, a layer of stressed silicon nitride was chosen as the structural layer, so the device is able to form its buckled shape after the final release by controlling the stress based on the parameters used during plasma enhanced chemical vapor deposition (PECVD). Figures 47 and 48 show the layouts of bottom and top electrode layers, respectively, for which molybdenum was chosen as the target material. Piezoelectric layer mask is shown in Figure 49.

Several materials were considered and tested for the piezoelectric layer and aluminum nitride was chosen as the final option for several reasons, which will be discussed in detail 
in the next section. Figure 50 shows the backside mask for releasing the deposited structure on the top of silicon wafer. Several other types of devices such as a long version of the current bistable system, clamped-clamped and clamped-free cantilever layouts were also design and patterned for each layer in the final masks to see the effects of the final residual stress in the whole structure after releasing the fabricated devices. All these layouts were designed in L-Edit software and the final photomasks were built at the University of Louisville Micro/Nano Technology Center [84]. In the next section, the microfabrication and characterization processes performed for making each layer of the MEMS device will be thoroughly discussed.

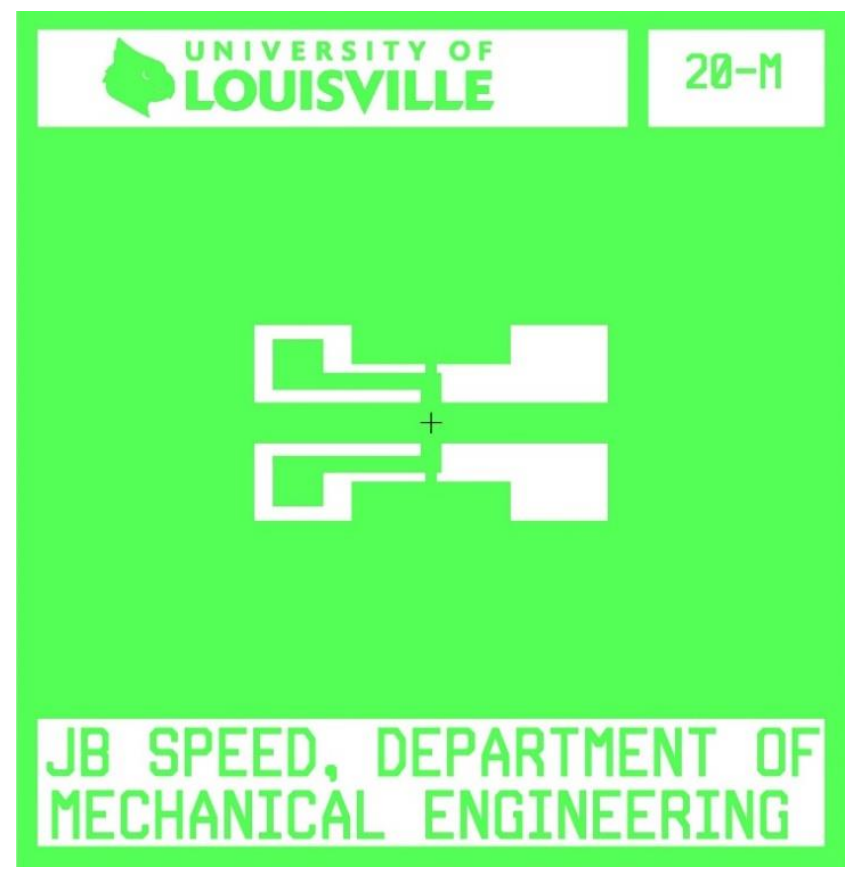

Figure 46. The photomask layout corresponding to the stressed silicon nitride as the structural layer of the device. 


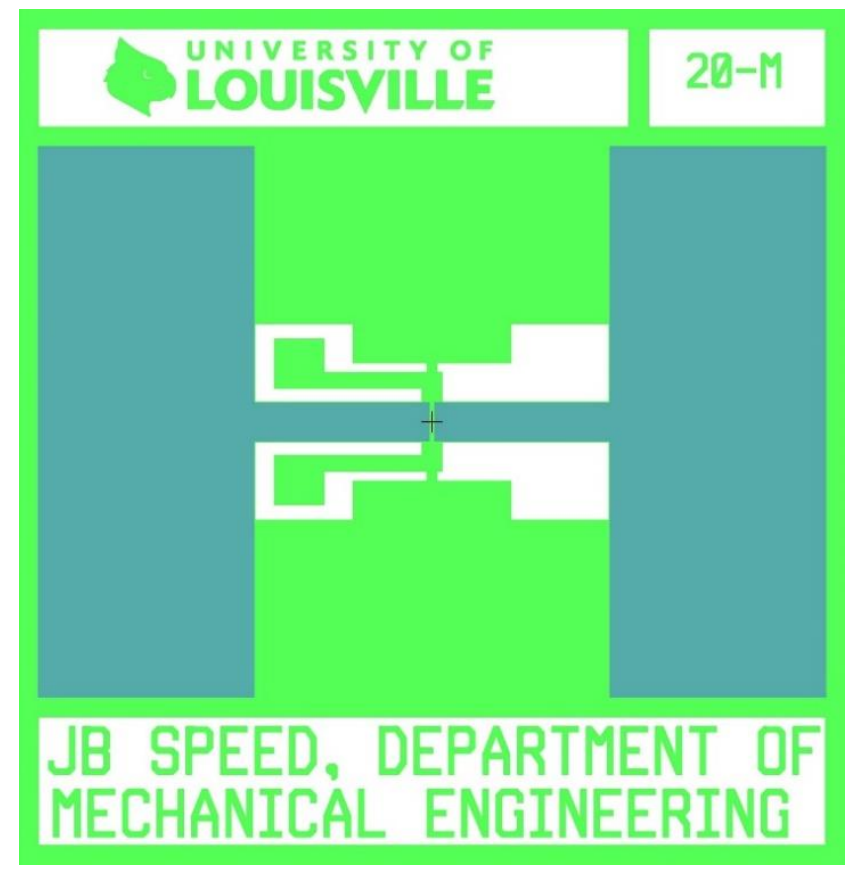

Figure 47. The photomask layout corresponding to the molybdenum as the bottom electrode layer of the device.

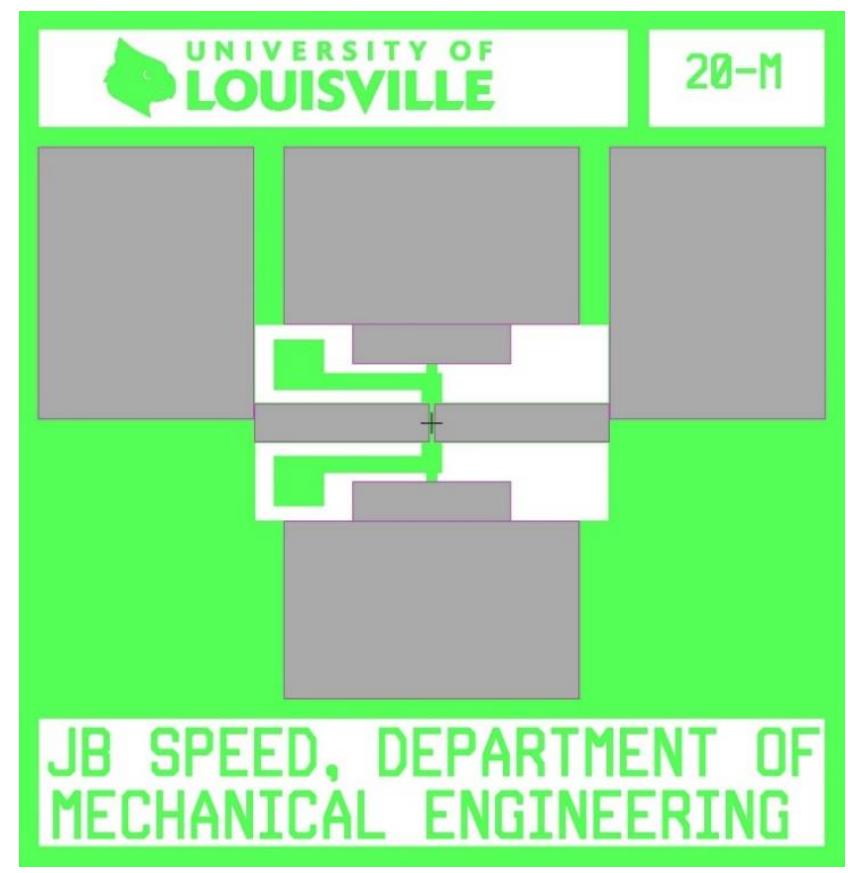

Figure 48. The photomask layout corresponding to the molybdenum as the top electrode layer of the device. 


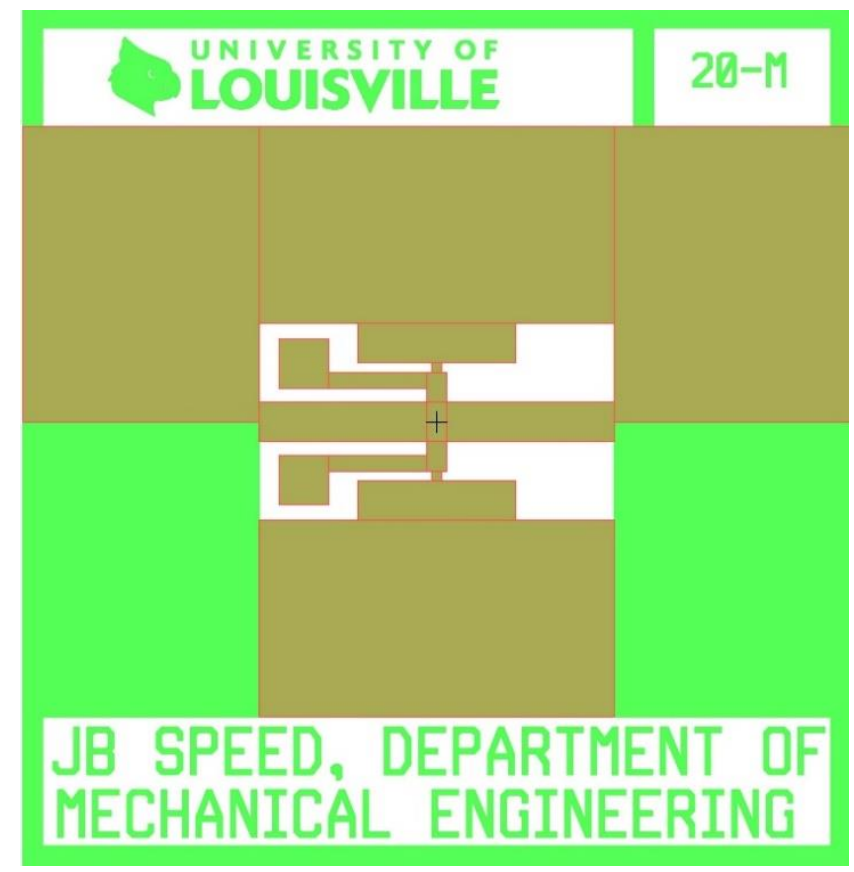

Figure 49. The photomask layout corresponding to the aluminum nitride as the piezoelectric layer of the device.

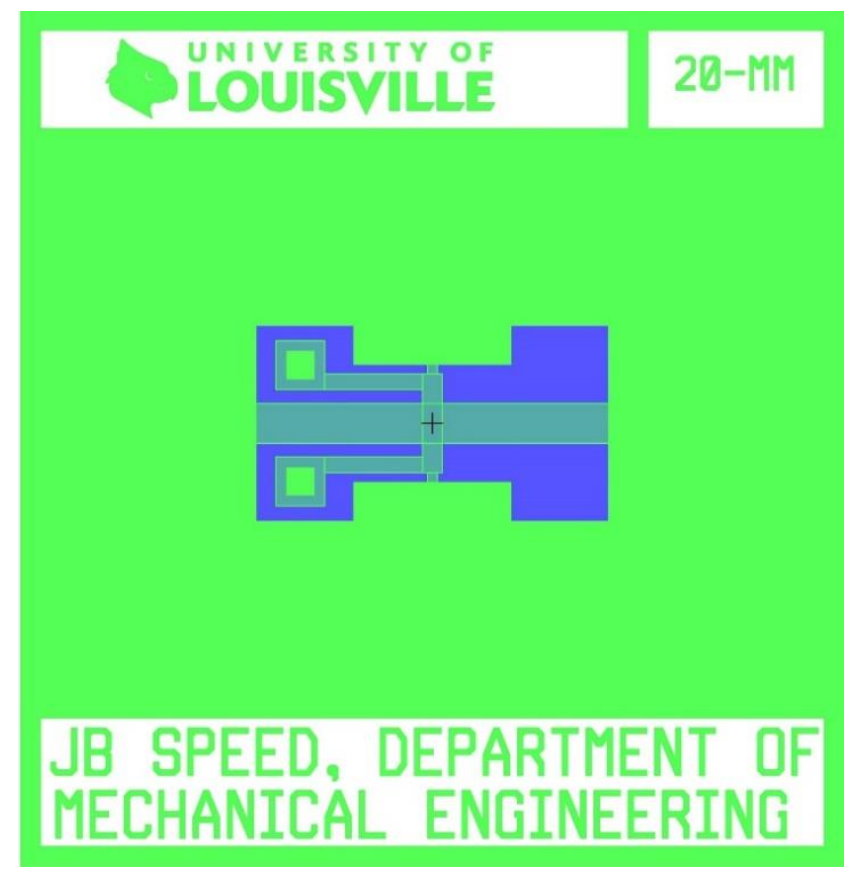

Figure 50. The photomask layout corresponding to the bulk etching of the top fabricated device in the DRIE process. 


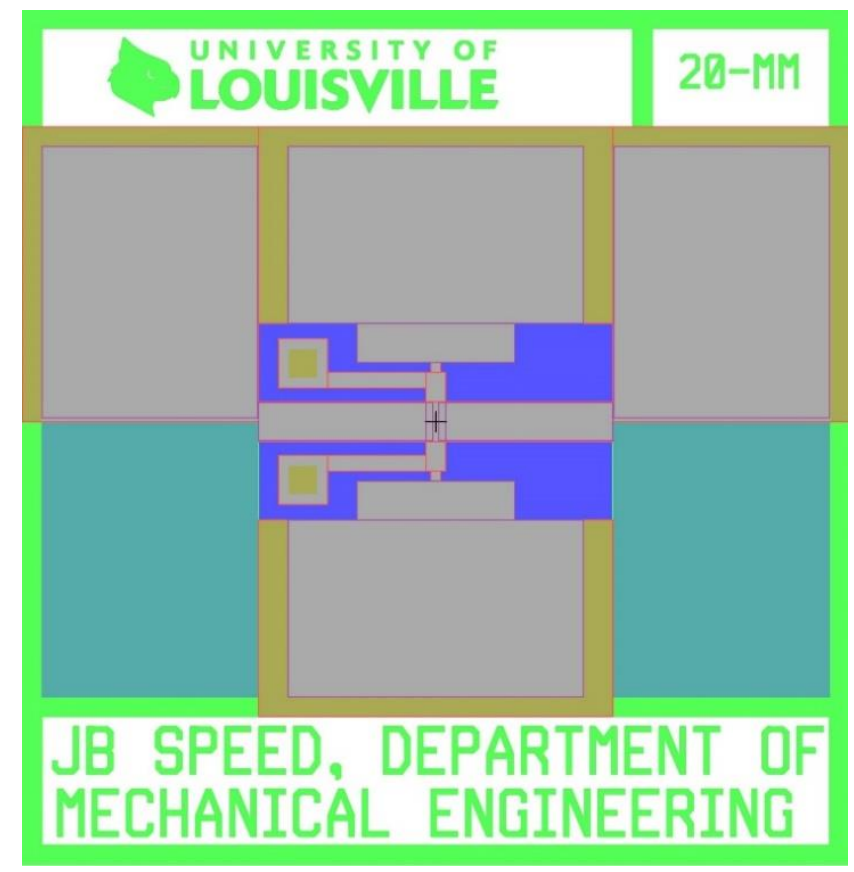

Figure 51. All five photomask layouts superimposed on the top of another.

\subsection{Microfabrication and Characterization}

In the previous section, the photomasks designed for fabricating the MEMS device were shown and discussed briefly. This section reviews all the microfabrication and characterization processes designed and performed to build the device. The wafers selected for doing all the experimental microfabrication processes were pure silicon wafers with $500 \mu m$ thickness and (100) crystalline plane orientation. Both sides of the wafer were covered with $2.5 \mu \mathrm{m}$ oxide layers to act as an etch-stop during the DRIE process, which were thermally grown on the silicon wafer (purchased commercially as grown). Since all the fabrication steps required photolithography, first, this process is explained thoroughly and then patterning and etching process of each layer of the device as well as final releasing the topside fabricated device from the silicon substrate are discussed individually.

\subsubsection{Photolithography}


In total, five photomasks were designed for microfabricating the studied device, four masks for the topside surface fabrication and one for the bulk etching of silicon from the backside. All the photolithography processes were performed in the photolithography bay located at the UofL Micro/Nano Technology Center. The masks with appropriate aligning markers were patterned and made on chrome coated glass. These masks fit within the SUSS Mask Aligner, which is used to align masks with existing features on the wafer and expose the photoresist deposited on a wafer to create patterned areas protected with photoresist.

The photolithography process includes spinning photoresist, soft baking, exposing photoresist using mask aligner, developing the exposed photoresist, and hard baking. For all the topside layers (except for the AlN layer where AZ P4620 was chosen as the photoresist), the standard Shipley 1827 positive photoresist was used, which estimated thickness was $2.7 \mu \mathrm{m}$. For patterning the backside of the wafer, AZ P4620 photoresist was used, which gives a relatively thick layer of photoresist (in our experiment the cured photoresist was measured between 6 to $8 \mu m$ ). This thick photoresist also played a role as the protective layer for the DRIE process.

Figure 52 shows one of the three Headway Spinners and the hotplate next to it which was used for covering the top surface of the deposited layer with a photoresist. After spinning the photoresist, the wafer was put either on the hotplate at $115^{\circ} \mathrm{C}$ temperature for 1 to 2 minutes or in the oven for 15 to 30 minutes to perform the soft baking process. Then the sample and the photomask were inserted into the SUSS Mask Aligner (Figure 53) for the alignment and exposure purpose. Depending on the type of photoresist a certain exposure time was used for each photolithography step. Since all the photoresists used in this experiment were positive, the next step was developing the exposed areas of photoresist. 
One minute of MF-319 developer and five minutes of AZ 400K developer was used for developing Shipley 1827 and AZ P4620 respectively. After development was done, the wafer was put in the Spin-Rinse-Dryer which was then followed by the hard baking process either on the hotplate for 5 to 10 minutes or in the oven for 30 minutes to 1 hour (depending on whether one or both sides of the wafer were covered with a photoresist). When the hard baking was done, the sample was ready for further processes.

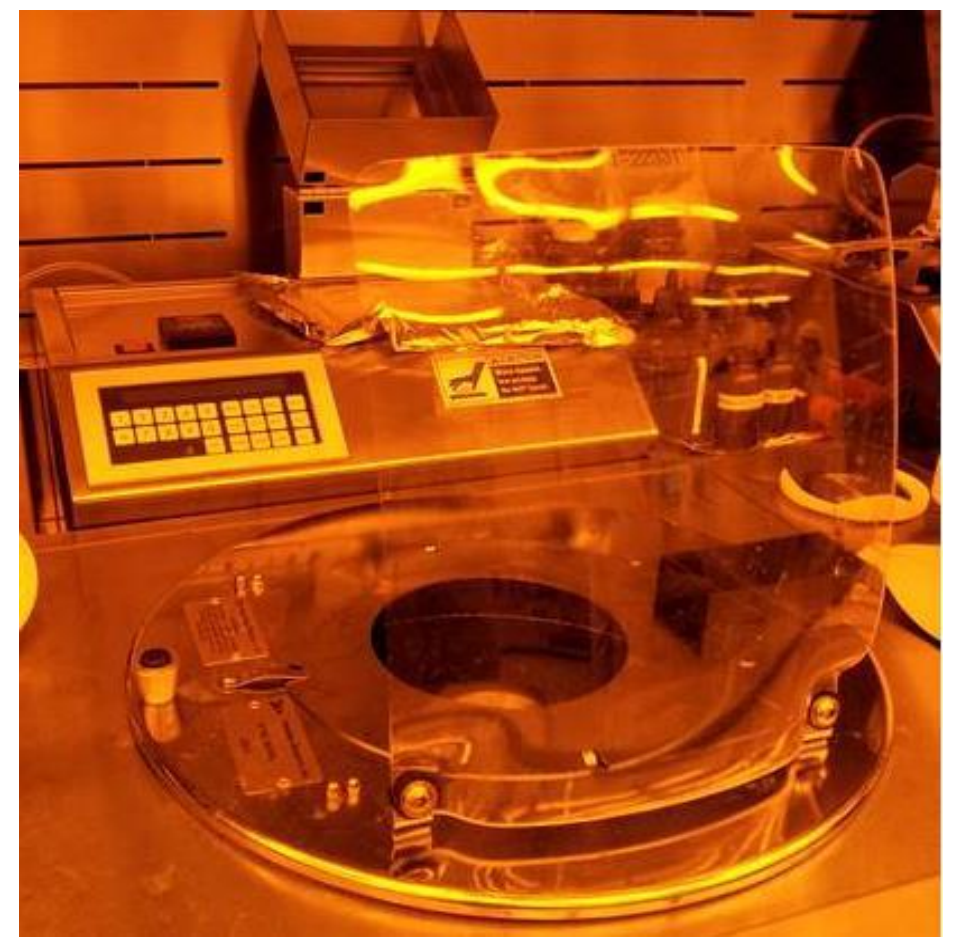

Figure 52. The Headway Spinner and the hotplate located in the photolithography bay at the UofL Micro/Nano Technology Center. 


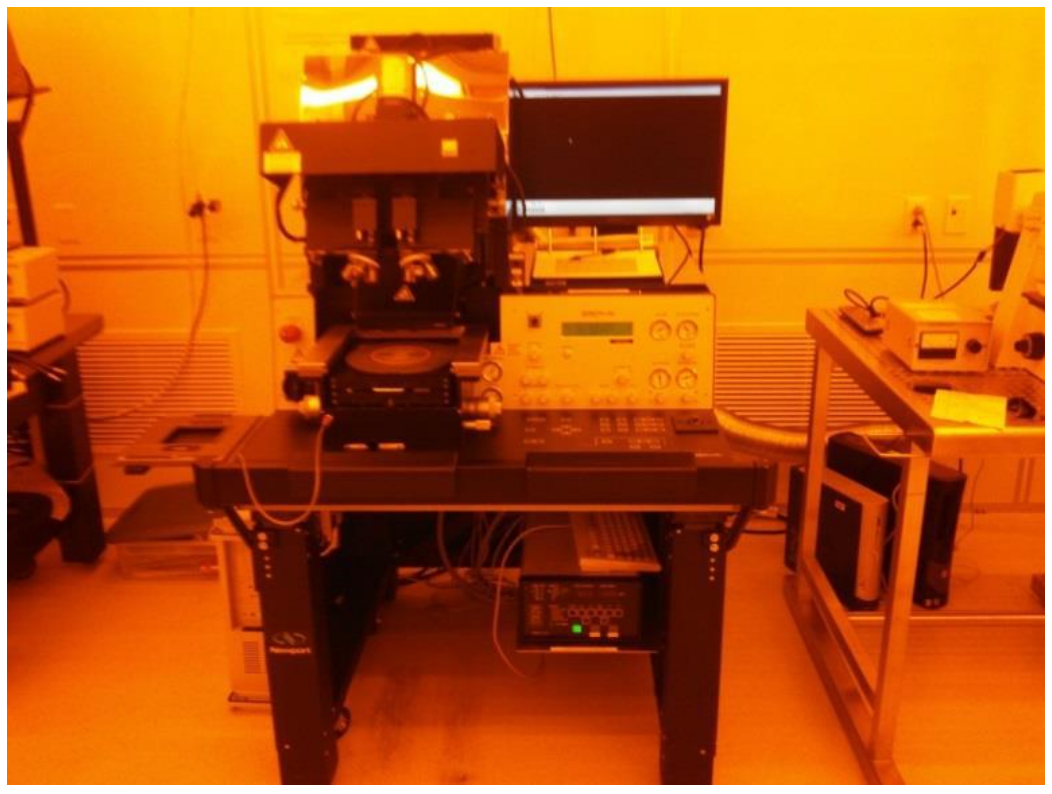

Figure 53. The SUSS Mask Aligner located in the photolithography bay at the UofL Micro/Nano Technology Center.

\subsubsection{Structural Layer}

A compressive residually stressed silicon nitride $\left(\mathrm{Si}_{3} \mathrm{~N}_{4}\right)$ was chosen to make the structural layer of the device. Since this is a bistable system, the compressive stress created in the structural layer causes the whole device to be buckled after the final release (Figure 54). For that purpose, plasma-enhanced chemical vapor deposition (PECVD), which is a chemical vapor deposition method to deposit thin films from a gas state (vapor) to a solid state on a substrate, was used to deposit the stressed nitride as the first layer of the device. Oxford PECVD PS100 (Figure 55) was used to deposit the stressed nitride layer where the amount of induced stress was mostly controlled by changing the heater and chiller temperature, high and low-frequency power, and the chamber pressure of the machine. To measure the stress of the deposited nitride layer, Toho FLX-2320-S (Figure 56), a thin film stress measurement equipment, was used. This machine works based on measuring the 
curvature change of pre and post-film deposition (it is a laser-based measurement device from the reflective area of the sample). The stress calculation is based on Stoney's equation, which relates the biaxial modulus of the substrate, thickness of the film and the substrate, and the curvature change, therefore the stress measurement process must be performed exactly before and after the film deposition in order to minimize possible stress variations due to any other processes.

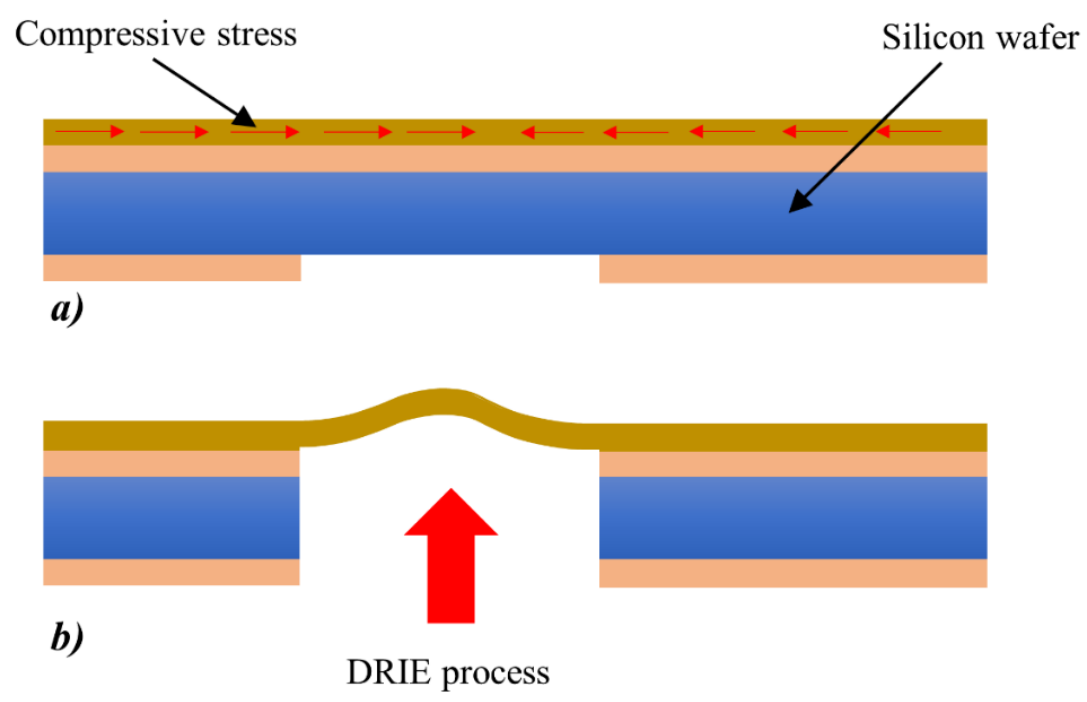

Figure 54. Schematic view of the fabricated device for DRIE process: a) before final release; b) after final release. 


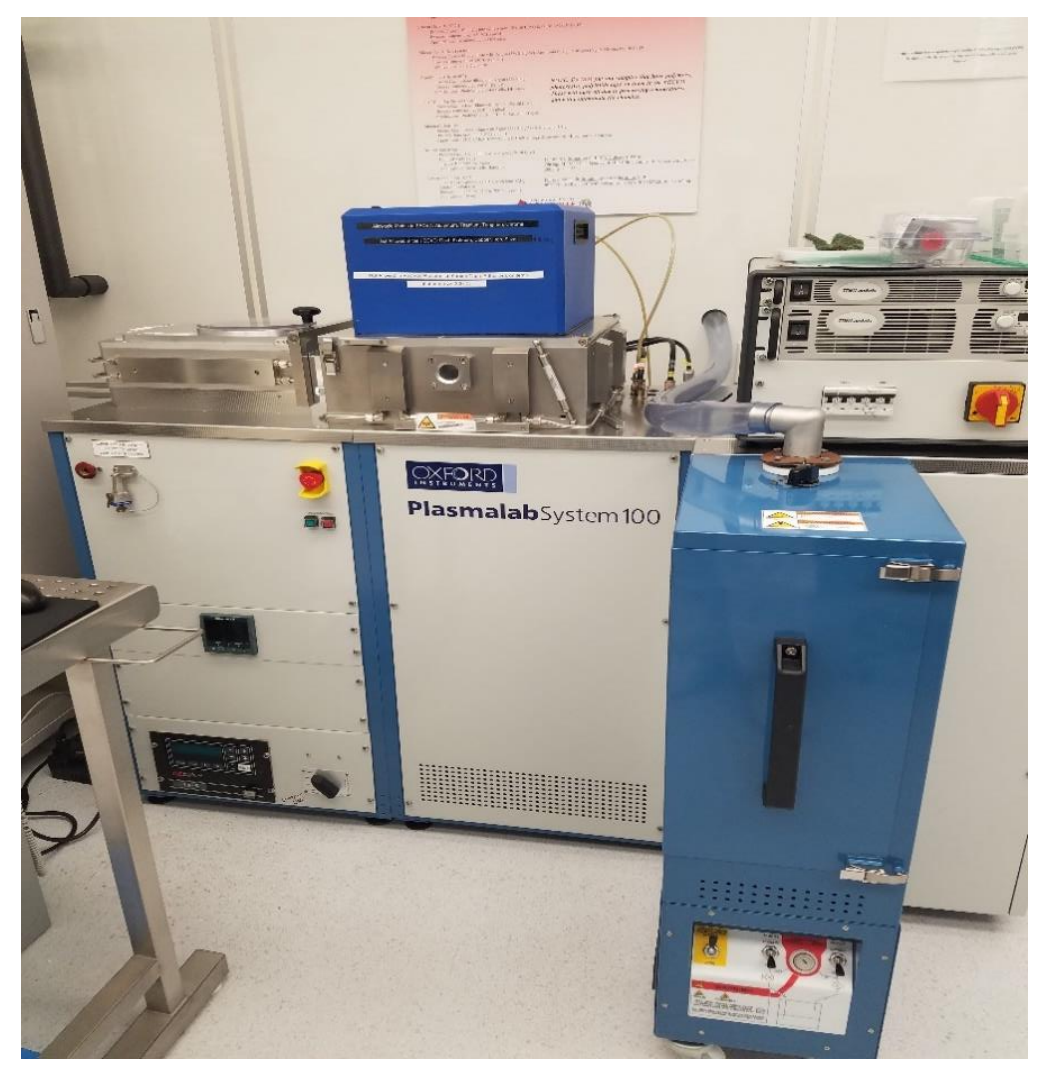

Figure 55. Oxford PECVD PS100 machine located in the deposition bay at the UofL Micro/Nano Technology Center.

The final recipe used for the deposition of stressed nitride includes $400 \mathrm{sccm}$ of $5 \%$ $\mathrm{SiH}_{4} / \mathrm{Ar}, 600 \mathrm{sccm}$ of $\mathrm{N}_{2}$, and $20 \mathrm{sccm}$ of $\mathrm{NH}_{3}$. It was performed at a chamber pressure of 850 mTorr with the heater and chiller temperatures set to $300{ }^{\circ} \mathrm{C}$ and $70{ }^{\circ} \mathrm{C}$ respectively. Both high and low-frequency power set to $50 \mathrm{~W}$ at 2 seconds pulse length. 50 minutes deposition of this recipe gave us 1 to $1.1 \mu m$ thickness of silicon nitride with the compressive stress ranging from 300 to $350 \mathrm{MPa}$. This amount of stress is much higher than what calculated for the critical stress in chapter five making sure that the bistability occurs after the final release. 


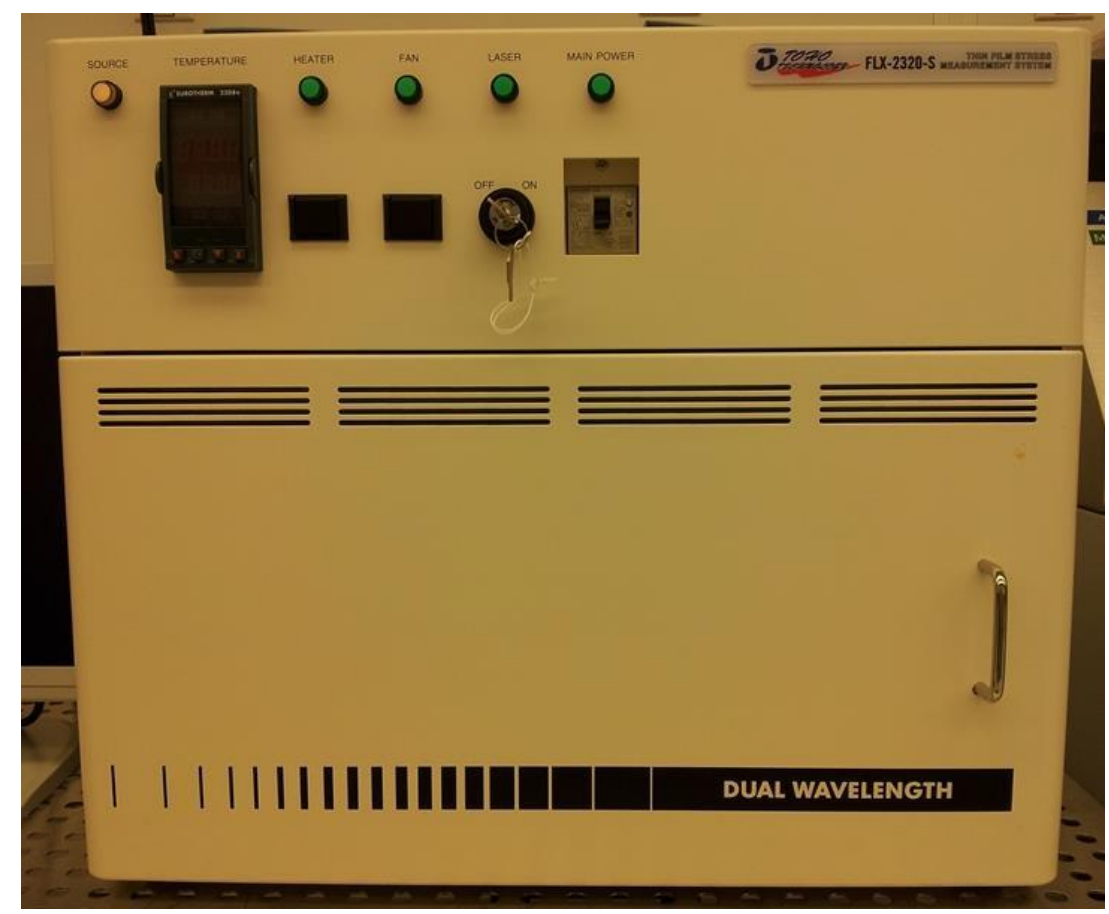

Figure 56. Toho FLX-2320-S thin film measurement machine located in the deposition bay at the UofL Micro/Nano Technology Center.

For each fabricated layer, an etching process must be performed after the photolithography to etch away the uncovered areas of the deposited film and pattern the structure of the device. In general, there are two etching methods: dry etching and wet etching process. In this study, both methods were used based on the layer material and its selectivity over the other existing layers in the sample. For the stressed silicon nitride, a plasma-based dry etching method was chosen to pattern the deposited layer. An ICP/RIE Trion Metal Etcher (Figure 57) was used to etch the PECVD deposited stressed nitride. The selected recipe includes $45 \mathrm{sccm}$ of $\mathrm{CH}_{3}$ and $5 \mathrm{sccm}$ of $\mathrm{O}_{2}$ with the power of $75 \mathrm{~W}$ RIE and $300 \mathrm{~W}$ ICP at a pressure of $50 \mathrm{mTorr}$ and the estimated etch rate with the DC bias voltage of $-100 \mathrm{~V}$ was measured around 35 to $40 \mathrm{~nm} / \mathrm{min}$. The dry etching recipe of silicon oxide and nitride are pretty similar, so this process might attack thermally oxide layer under the silicon 
nitride as a result of over-etching process. However, it was not considered causing any major issues as the measured etch rate was pretty low and the existing oxide layer was thick enough and more resistant to the dry etching process than the PECVD deposited stressed nitride layer.

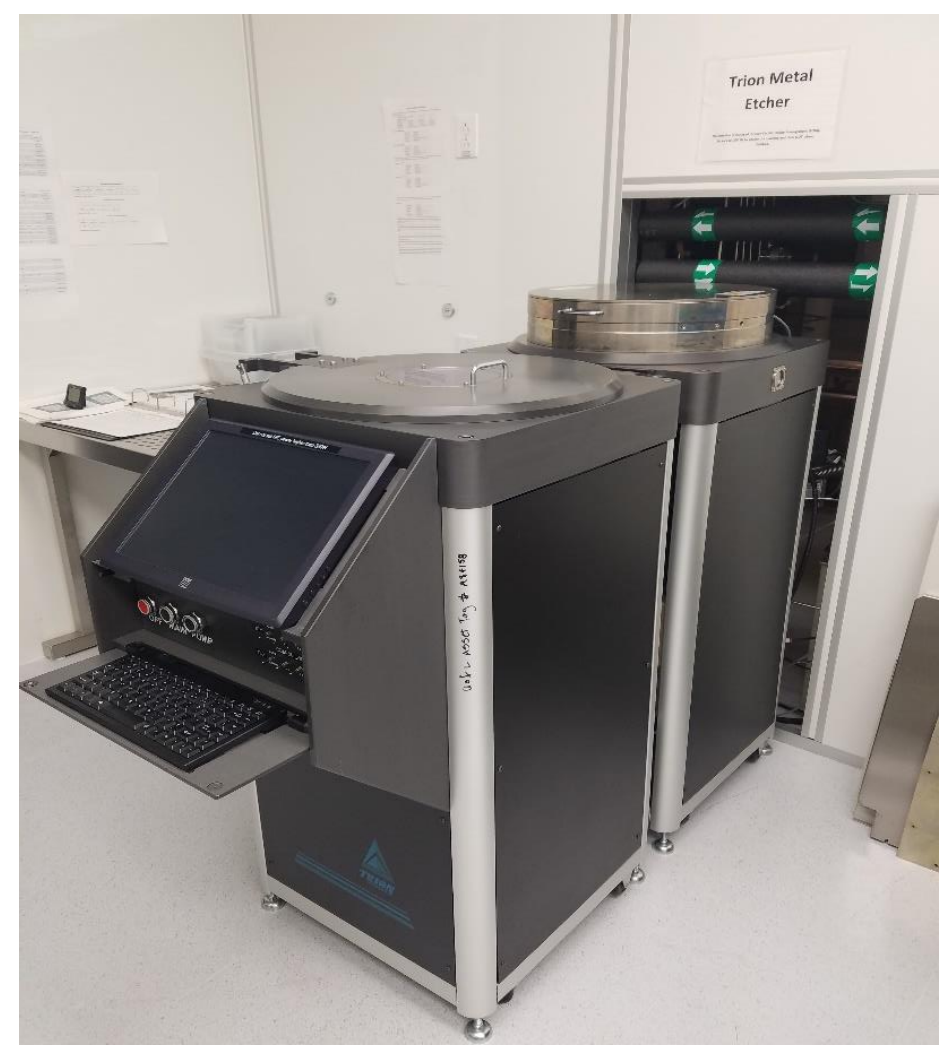

Figure 57. ICP/RIE Trion Metal Etcher located in the etching bay at the UofL Micro/Nano Technology Center.

\subsubsection{Electrode Layers}

A piezoelectric energy harvester requires conductive layers to transfer the created electrical current to the external load. In this proposed energy harvester, deposited metal layers play as the bottom and top electrodes of the device in a way that the piezoelectric layer is sandwiched between them. For both bottom and top electrodes, molybdenum was chosen 
as the deposited material. The reason for that is the ease of its deposition process and its relatively high density, which helps lower the first natural frequency of the system.

The deposition of molybdenum was performed by the physical vapor deposition (PVD) process. In this plasma-assisted sputtering method, the material target and the substrate are inserted in the machine with a certain distance between them. The deposition takes place as a result of ejecting material from the target (in this case molybdenum) to the substrate. This ejection happens due to the bombards of positively charged argon gas toward the target material which is initiated by the plasma discharge localized around the target by a magnet. These ejected material atoms from the target travel to the substrate at a very low pressure to complete the deposition process [85].

Figure 58 shows the Kurt J. Lesker PVD 75 Sputtering machine used in this study to deposit both electrode and piezoelectric layers of the device. The molybdenum deposition was performed using a 4-in pure target material supplied with a power of $500 \mathrm{~W}$. The chamber was pumped down for at least 45 minutes and the process was performed at a constant pressure of 6 mTorr. The deposition rate measured for this recipe was around 25 to 30 $\mathrm{nm} / \mathrm{min}$. In this study, a $300 \mathrm{~nm}$ thick molybdenum layer is sufficient for the electrode purpose. However, due to the possibility of being over-etched in the further processes, a thicker molybdenum layer ( 450 to $600 \mathrm{~nm}$ ) was deposited for both the bottom and top electrodes. 


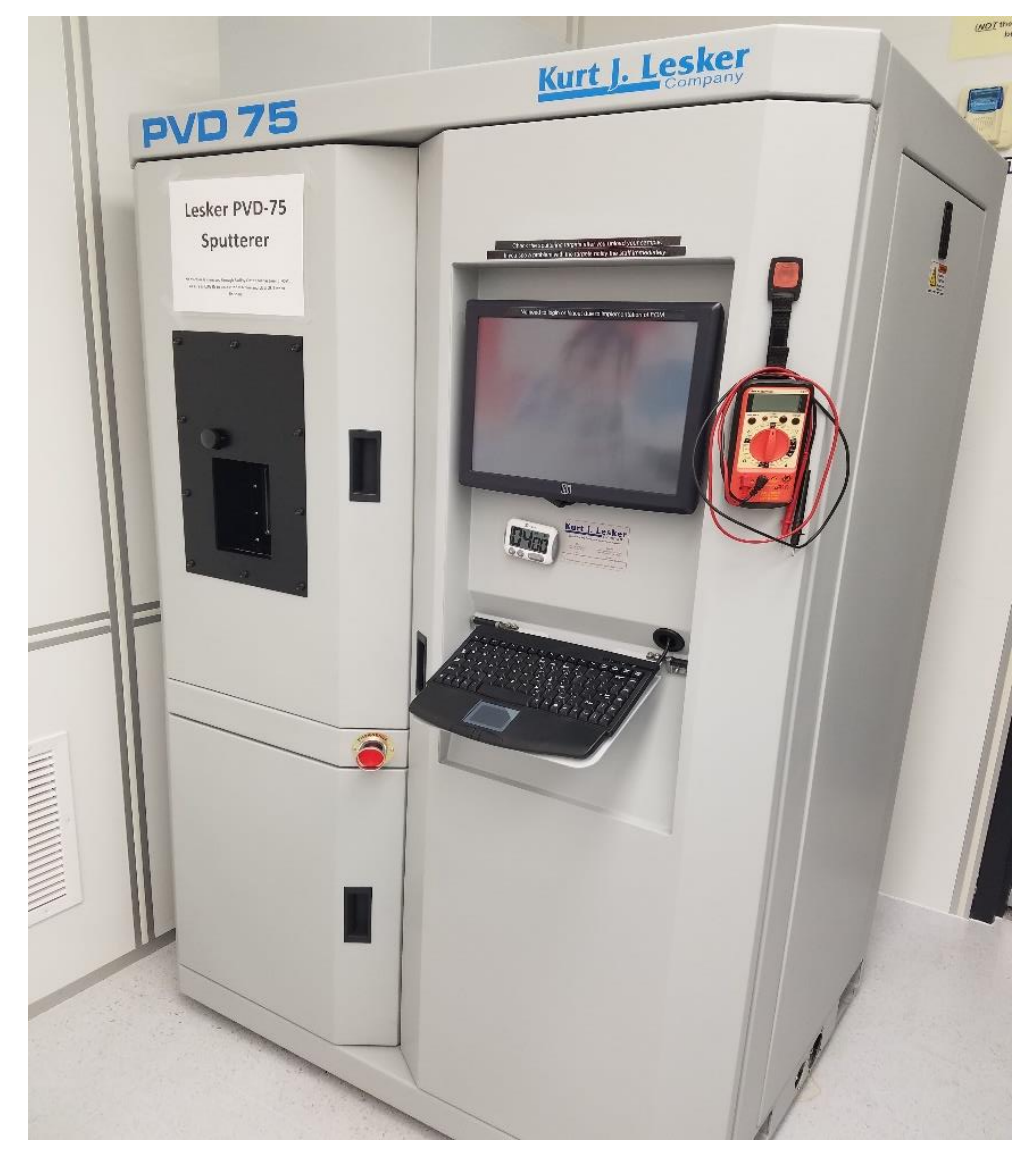

Figure 58. Kurt J. Lesker PVD 75 Sputtering machine located in the deposition bay at the UofL Micro/Nano Technology Center.

Etching of the patterned molybdenum layer was performed with the Trion Metal Ether. Cured Shively 1827 photoresist was used as the protective layer in the etching process. 20 sccm of $\mathrm{Cl}_{2}, 5 \mathrm{sccm}$ of $\mathrm{O}_{2}$, and $5 \mathrm{sccm}$ of Ar were used with $50 \mathrm{~W}$ RIE and $300 \mathrm{~W}$ ICP power at a chamber pressure of 30 mTorr. This recipe etches molybdenum at a pretty high rate, between 80 to $100 \mathrm{~nm} / \mathrm{min}$, which requires having a thicker layer of molybdenum, especially for the top electrode, to avoid losing electrical conductivity of the bottom electrode as a result of being over-etched.

\subsubsection{Piezoelectric Layer}


The piezoelectric layer is the most important part of the MEMS device as it provides the essential function of the energy harvester which is converting the mechanical energy into the electrical one. Therefore, a detailed study is required for possible ways to fabricate this layer of the device. Among all the piezoelectric materials, lead zirconate titanate (PZT) has the highest piezoelectric coefficients and is known as the most reliable material to work as an energy harvester; however, its deposition needs to be processed at a pretty high temperature (above $500{ }^{\circ} \mathrm{C}$ ) $[86,87]$. This high temperature could possibly destroy all the created stress in the silicon nitride as a result of annealing post-process. To that end, in this study, two other options were considered for the deposition as the piezoelectric material: aluminum nitride and gallium nitride. Due to the several complexities of gallium nitride deposition at low temperatures, which will be discussed later in this section, aluminum nitride was chosen as the final material for the piezoelectric layer.

There are several studies on the deposition of aluminum nitride at low temperatures [88, 89]. The piezoelectric properties of AlN are dependent on the crystal orientation of the film, which in turn is related to the condition of the deposition process. As shown in the previous studies, growing AlN with a $c$-axis (002) orientation is necessary to have piezoelectric effects [90]. Growing a certain crystal orientation in a film is related to several parameters of the deposition process including but not limited to substrate material, sputtering temperature, electrical power, chamber pressure, flow rate of the added gases, distance between the target and the substrate, etc. [91-95].

Huang et al. [96] investigated AlN thin film deposition on the molybdenum as a seed layer under various conditions using reactive RF magnetron sputtering. The influence of RF power and nitrogen flow rate was studied on the AlN film properties. The optimal RF power 
of $400 \mathrm{~W}$ and a nitrogen flow rate of $75 \%$ was found to grow the preferred $c$-axis oriented AlN on the molybdenum substrate. Akiyama et al. [97] also studied the preparation of highly oriented aluminum nitride thin films on the molybdenum substrate by using metal interlayers. They showed that using $\mathrm{Au} / \mathrm{Ti}$ interlayers between AlN and Mo substrate could potentially improve the crystal orientation of the deposited film. Aluminum nitride thin film deposition with DC pulsed magnetron sputtering process was reviewed in [98] on several seed layers at various sputtering conditions. Calculation of $d_{31}$ and $d_{33}$ for an AlN thin layer was determined for several sputtering conditions and it was shown that using various substrates as seed layers could significantly affect a deposited thin film piezoelectric coefficients.

While aluminum nitride has been considered in several studies for energy harvesting purposes $[99,100]$ due to its easy deposition process and its relatively high piezoelectric properties, gallium nitride is mostly known for its use in light-emitting diode (LED) semiconductors [101, 102]. Rais-Zadeh et al. [103] investigated various parameters of GaN as an electromechanical material. They reviewed $\mathrm{GaN}$ and $\mathrm{Al} / \mathrm{GaN}$ mechanical and electrical properties with the emphasis of their potential use in NEMS/MEMS devices. It was shown that GaN-based devices could be a potential option for the next generation of low-noise, high-speed microelectromechanical devices. A GaN p-n junction piezoelectric generator was studied in [104] to be used as an energy harvester. A highly efficient GaNbased energy harvester was fabricated and its performance was evaluated by charging a commercial capacitor. The encouraging results in this study showed that GaN p-n junctions could make a huge contribution to the future of both piezo-phototronic and energy harvesting devices. 
In this experiment, the deposition of aluminum nitride was done with Kurt J. Lesker PVD 75 Sputtering machine. The initial recipe was picked from [96] and a previous study by this group [105]. The first run of AIN deposition was performed by using a 4-in pure aluminum target. The chamber pressure was pumped down for at least one hour and the substrate temperature was set to $300^{\circ} \mathrm{C}$. Sputtering was performed by applying a power of $500 \mathrm{~W}$ and adding nitrogen and argon gases with a mixed ratio of 3:1 at a constant flow rate of $20 \mathrm{sccm}$ nitrogen and $7 \mathrm{sccm}$ argon. The experimental observations showed that adding a high rate of nitrogen gas could significantly increase the deposition time due to the relatively high pressure generated in the chamber during the process. It could also unstabilize the process as a result of an electrical shorting occurrence between the target and the dark space shield in the sputtering machine which consequently causes the plasma not functioning properly. To avoid this issue, a lower flow rate of mixed gases $(9 \mathrm{sccm}$ of $\mathrm{N}_{2}$ and $3 \mathrm{sccm}$ of Ar) was tested while the mixed ratio was kept the same. A noticeable improvement was observed as a result of this change in both the deposition rate and process stability.

Based on $[92,93]$, lower sputtering pressure and shorter distance between the substrate and the target could help grow (002) oriented AlN, while higher pressure and a longer gap between the target and the substrate could lead to growing (100) oriented AlN. Another point needs to be considered in aluminum nitride deposition is that growing (002) crystal orientation requires higher power. Unfortunately, Kurt J. Lesker PVD 75 Sputtering equipment used in this study has some major restrictions. There is a huge gap between the source and the substrate, which is fixed and not adjustable. Another issue is $500 \mathrm{~W}$ maximum power limitation set for this machine which makes growing (002) $c$-axis 
orientation in the $A l N$ sputtering process more challenging. These two factors left us with changing the added gas flow rates to keep the processing pressure as low as possible and the substrate temperature. As discussed earlier in this chapter, one of the main reasons for choosing aluminum nitride is the possibility of its deposition at low temperature to avoid losing much of the induced stress in the structural layer as a result of post-processing. To that end, several tests were performed at slightly higher temperatures $\left(350\right.$ and $\left.400{ }^{\circ} \mathrm{C}\right)$ and the results showed not a major difference in the obtained crystal orientation of the deposited film.

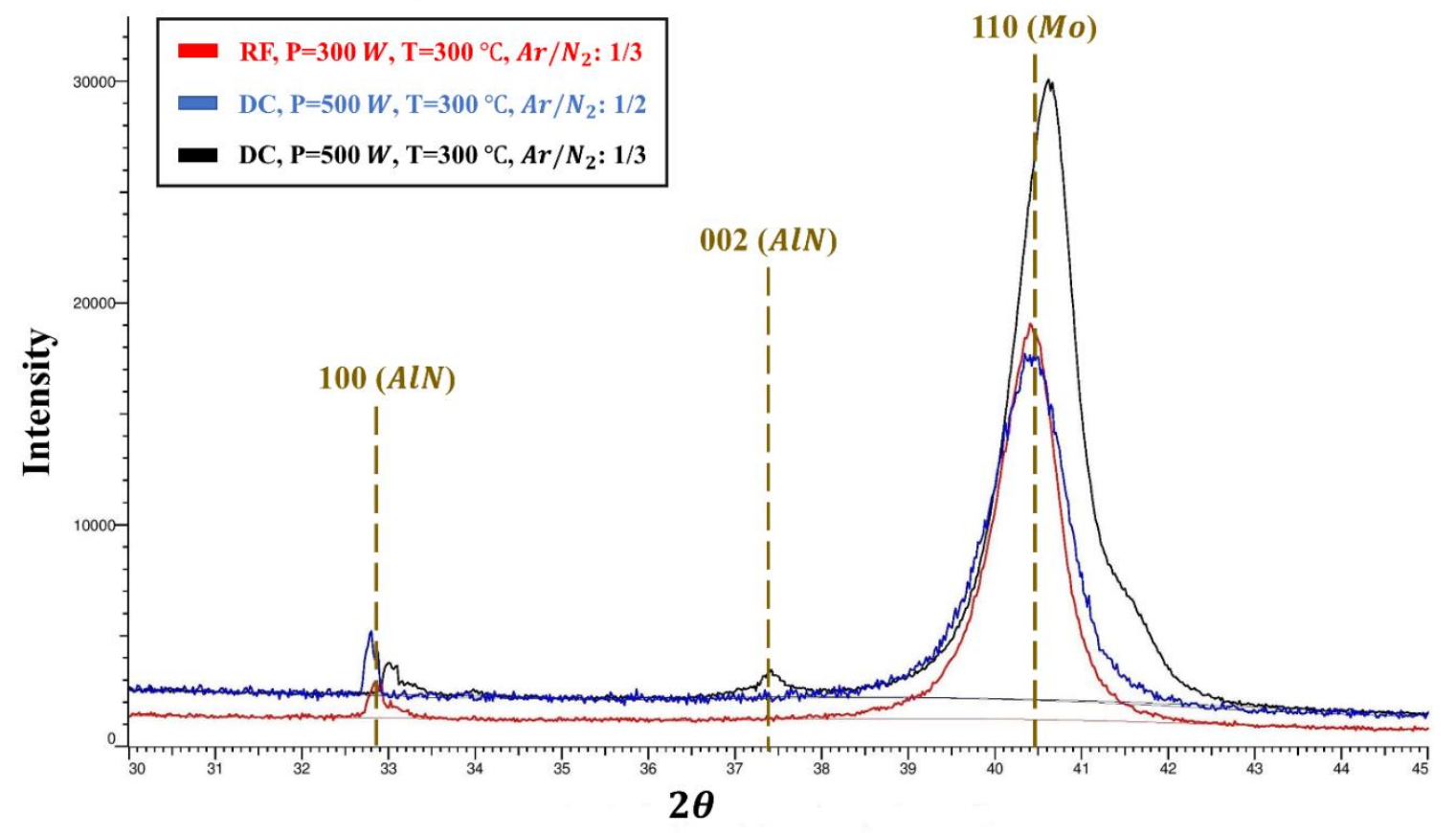

Figure 59. XRD results for three samples of aluminum nitride thin film deposited on a molybdenum substrate with various sputtering parameters.

Several samples of aluminum nitride thin film deposited on a molybdenum substrate were prepared and their crystal orientations were characterized by X-ray powder diffraction (XRD) technique. The X-ray diffraction test was performed with the collaboration of the 
Conn Center for Renewable Energy Research [106] at the University of Louisville. Figure 59 shows the XRD results of three samples grown on a molybdenum substrate with various processing conditions. Since there are several studies in which AlN was deposited with an RF sputtering method $[96,107,108]$, one of these three samples were grown under RF sputtering conditions. In all these three processes, the power was increased step by step gradually from a lower initial value to the final set, which turned out to be helpful to avoid having instability during the process. As can be seen from the plot, only one of these samples has (002) crystal orientation, the preferred $c$-axis orientation for having $d_{31}$ piezoelectricity, in which the sputtering parameters are: $500 \mathrm{~W}$ DC power, $\mathrm{N}_{2} / \mathrm{Ar}$ mixed ratio of 3:1 (9 sccm of $\mathrm{N}_{2}$ and $3 \mathrm{sccm}$ of $\mathrm{Ar}$ ), and the substrate temperature of $300{ }^{\circ} \mathrm{C}$, which were chosen as the optimal parameters for the aluminum nitride deposition in this study. Growing aluminum nitride piezoelectric layer is a pretty slow process that requires a whole day for preparing one sample. Further tests taken by a Veeco Dektak 8M Profilometer showed two hours of deposition with the optimal recipe gives us 500-600 $\mathrm{nm}$ thick aluminum nitride layer.

To experimentally evaluate the effects of the substrate material on the crystal orientation of aluminum nitride [109], a thin layer of platinum was deposited on a molybdenum substrate as the seed layer of aluminum nitride deposition. For that purpose, a mixed layer of Pt/Ti was deposited on a molybdenum substrate with Kurt J. Lesker PVD 75 Sputtering machine. Titanium was used as an adhesive layer for platinum deposition, therefore both targets must be loaded into the machine for the deposition process. The machine was pumped down for at least 45 minutes and the DC sputtering process was performed at a power of $300 \mathrm{~W}$ and a chamber pressure of $5 \mathrm{mTorr}$. First, Ti was deposited for 2 minutes, 
then Pt was sputtered for 3 minutes after the machine was rested for one minute in between, which gave us a thin layer with an expected thickness of $100 \mathrm{~nm}$. However, further XRD characterization results showed no success in growing (002) oriented AlN, which again is mostly due to the limitations of the PVD sputtering machine mentioned earlier in this section.

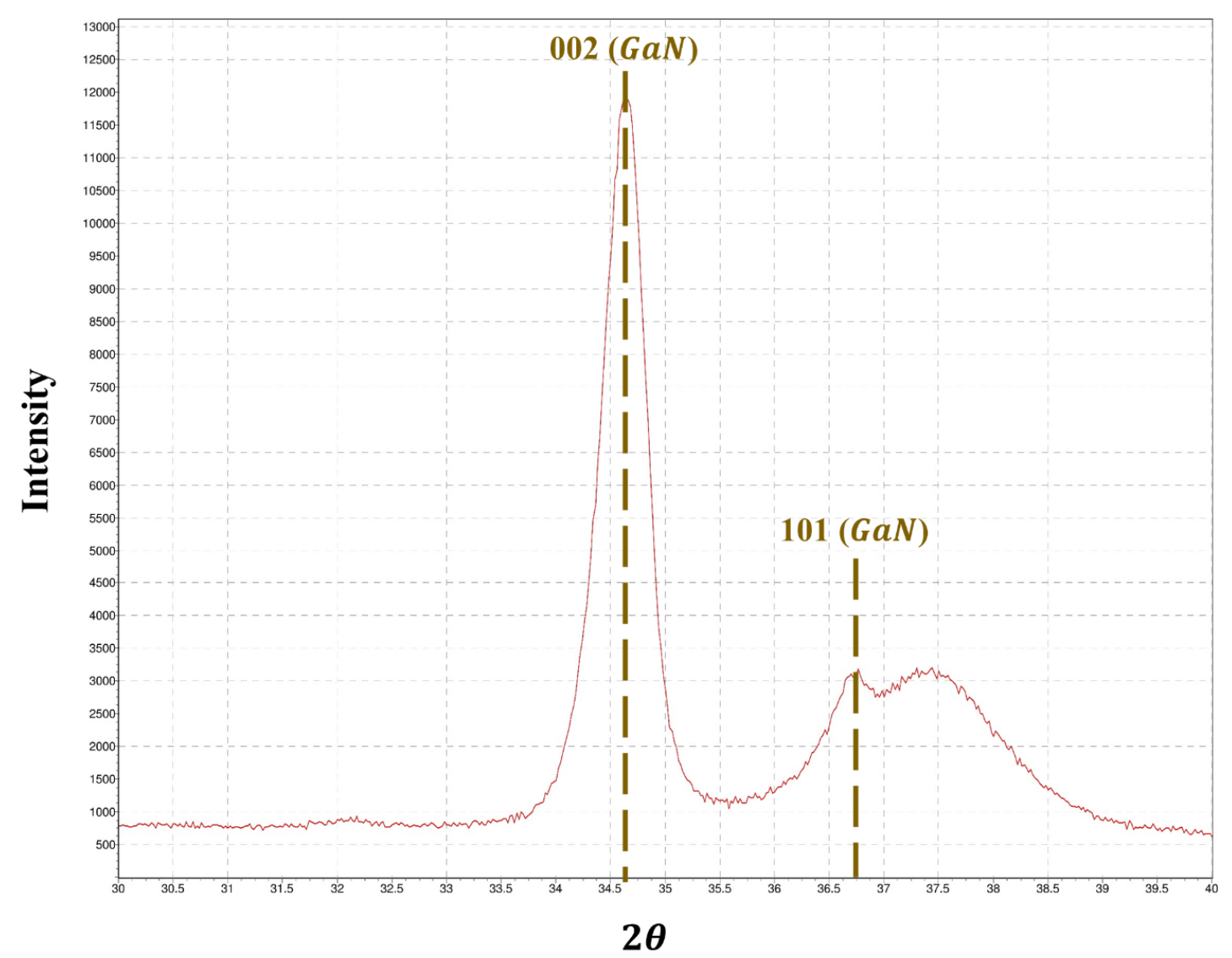

Figure 60. XRD results for the MOCVD deposited gallium nitride on a molybdenum substrate.

As discussed earlier, gallium nitride was another option considered in this study for a potential piezoelectric layer of the device. The deposition of GaN was performed with a metal-organic chemical vapor deposition (MOCVD) process at the Conn Center for 
Renewable Energy Research. After running some trials, GaN was deposited on three different substrates: $\mathrm{Si}, \mathrm{Si}_{3} \mathrm{~N}_{4}$, and Mo. X-ray diffraction results showed a strong peak of (002) crystal orientation in the deposited GaN layer for all these three samples. Figure 60 shows the XRD result of the GaN sample grown on a molybdenum substrate. As can be seen from this plot, there is a strong peak between 34 and 35 degrees which stands for (002) crystal orientation in GaN (as mentioned, this peak was seen for all three prepared samples and to simplify, only of them is shown here). However, MOCVD deposition of GaN was performed at a pretty high temperature $\left(550^{\circ} \mathrm{C}\right)$, which led us to choose the PVD sputtered aluminum nitride as the selected material for the piezoelectric layer of the device.

After the deposition of aluminum nitride as the piezoelectric layer, the wafer needs to be patterned and etched to be prepared for the top electrode deposition. There are two options to etch aluminum nitride: dry and wet etching process. Dry etching of AlN was first tried with the Trion Metal Etcher by using the recipe found in the previous study [105]. However, the measured etching rate was very low and it attacks the molybdenum layer, which is the bottom electrode of the device, at a very high rate $(100 \mathrm{~nm} / \mathrm{min})$. After running more trials by changing different parameters, the optimal recipe was found for dry etching of aluminum nitride as $14 \mathrm{sccm}$ of $\mathrm{Cl}_{2}, 10 \mathrm{sccm}$ of $\mathrm{BCl}_{3}$, and $6 \mathrm{sccm}$ of $\mathrm{Ar}$ at a chamber pressure of $20 \mathrm{mTorr}$ and ICP and RIE power of $500 \mathrm{~W}$ and $100 \mathrm{~W}$ respectively. This obtained recipe etches AlN at a pretty low rate of $20 \mathrm{~nm} / \mathrm{min}$ while it etches Mo at a rate of $30 \mathrm{~nm} / \mathrm{min}$, which is still higher than AIN etching rate and shows that ICP/RIE dry etching of AlN is not very selective over Mo. To that end, wet etching of AlN was considered as an alternative solution to this problem. In [110], it was shown that AZ 400K developer, in which the active component is $\mathrm{KOH}$, has the potential for etching AlN. A 
solution of $\mathrm{AZ} 400 \mathrm{~K}$ developer and distilled water with the mixed ratio 1:4 was prepared and tried on a sample of deposited AlN. It was observed that AZ 400K developer etches AlN at a rate of $30 \mathrm{~nm} / \mathrm{min}$, while it barely attacks Mo, which shows its high selectivity and led us to choose this process over dry etching for the piezoelectric layer.

\subsubsection{Bulk Etching and Final Release of The Device}

After all the topside fabrication is done, the next step is to pattern and etch the bulk silicon from the backside of the wafer to release the MEMS bistable devices. Wet etching of silicon with potassium hydroxide $(\mathrm{KOH})$ was studied in the previous efforts by this group [105]. Since $\mathrm{KOH}$ is an anisotropic etching process, it was found very difficult to release successfully the topside fabricated bistable devices by going through the whole thickness of the wafer. Therefore, in this study deep reactive ion etching (DRIE) process was selected as an alternative way to do the bulk etching of silicon. The main restriction for doing the DRIE process was the possibility of the equipment (Figure 61) being exposed to the existing metal layers fabricated in the wafer during the process, which may cause some major issues to the machine. To avoid this issue, a silicon wafer with pretty thick oxide layers $(2.5 \mu \mathrm{m})$, which helps protect the machine from being exposed to the top metal layers, was selected for this experiment.

The process began with the photolithography on the backside of the wafer. As mentioned earlier, AZ P4620 was selected as the photoresist for doing the backside photolithography, which played as the protective layer for the unexposed areas in the DRIE process. The topside of the wafer was covered with a Sively 1827 photoresist to be protected from any possible scratches in the post-processing. A backside aligning was also performed by the Suss Mask Aligner to make sure that the patterned backside photoresist was carefully 
aligned with the topside silicon nitride layer. To move forward with the DRIE process, first, the oxide layer at the backside of the wafer must be removed, which was performed by the buffered oxide etching (BOE) process. The BOE solution used for this process comprises a 6:1 volume ratio of $40 \% \mathrm{NH}_{4} \mathrm{~F}$ in water to $49 \% \mathrm{HF}$ in water. The measured etch rate for the thermally grown oxide layer was about $100 \mathrm{~nm} / \mathrm{min}$, which indicates that 25 minutes of an etching process is required to fully etch the oxide layer from the sample.

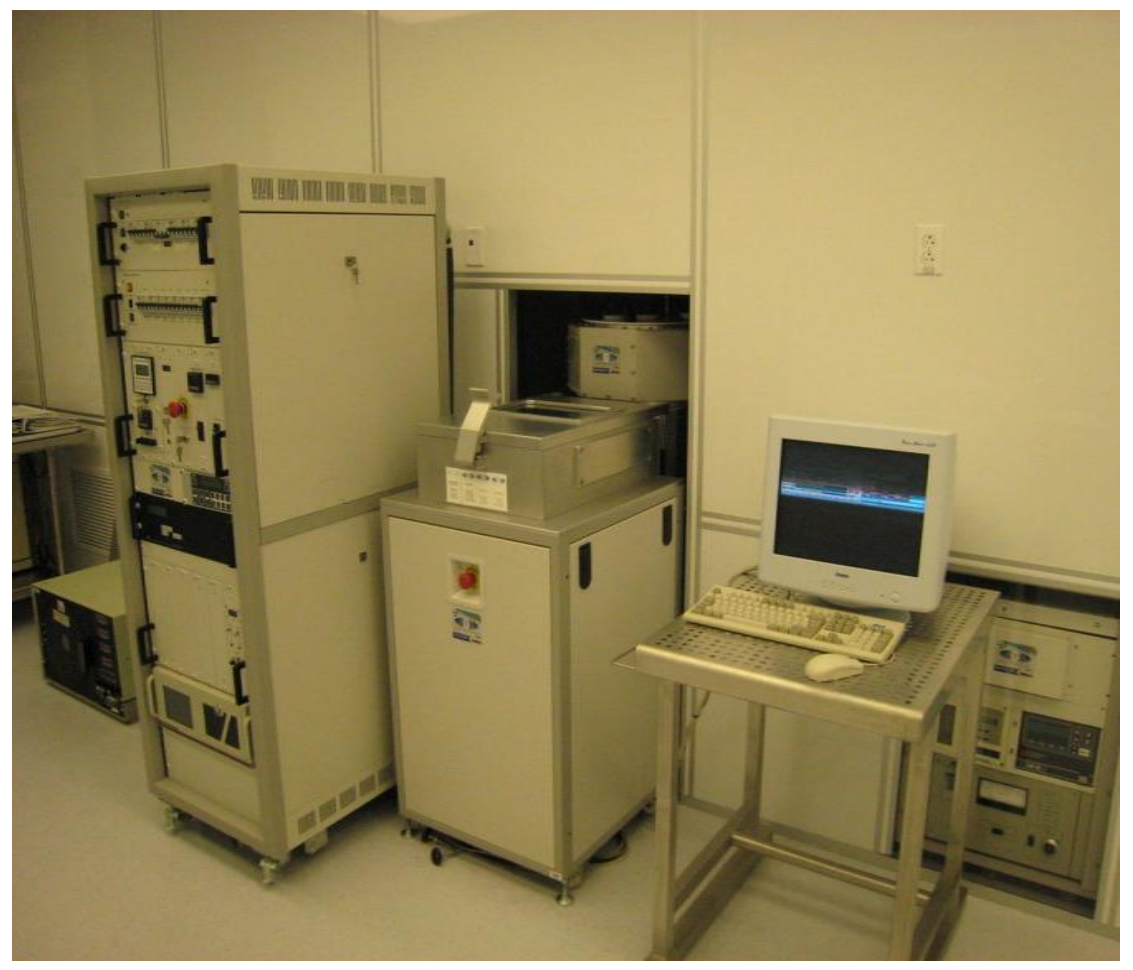

Figure 61. Deep reactive ion etching (DRIE) machine located in the etching bay at the UofL Micro/Nano Technology Center.

Since we intended to etch through the whole thickness of the wafer in the DRIE process, we needed to bond the sample with a supporting wafer to avoid it from getting broken in the processing chamber. A typical way to attach two wafers together is by using crystal bond adhesive. However, in this way, the debonding process is done by sliding the 
supporting wafer from the sample at a high temperature $\left(100^{\circ} \mathrm{C}\right)$, which may result in breaking the final released devices. As an alternative option, we decided to bond the supporting wafer by using a photoresist. A layer of Shively 1827 photoresist was spun on the supporting wafer and the sample was attached to it from its topside surface and then bonded wafers were cured in the oven at $115^{\circ} \mathrm{C}$ temperature for an hour. The whole DRIE processing time required to etch through all the silicon in the wafer is about 2 hours for the default recipe of the machine. However, since the DRIE etching process is not completely linear, it was performed in multiple steps and the etching depth was measured by the Dektak Profilometer after each step to make sure that the sample was not getting over etched.

After finishing the DRIE, the sample was processed in the $\mathrm{Xactix}_{\mathrm{XeF}}$ machine, which is a selective dry etching system for silicon, to make sure that any remaining silicon was etched away from the backside of the wafer. The next step was to etch the oxide layer at the topside of the wafer, so the fabricated devices could be released from their substrates. Since the first fabricated layer of the device was stressed silicon nitride, for which the etching recipe is pretty similar to the one being used for an oxide layer, the challenging part of this step was to etch the oxide layer of the wafer without attacking the stressed silicon nitride. Both dry and wet etching processes were tested to measure the etching rate and selectivity for the oxide layer. Since the designed bistable devices are pretty thin with considerable induced stress in the structural layer, the number of post-processing steps must be minimized at the final stage to reduce the chance of breaking devices after detaching the supporting wafer. This led us to first try the dry etching process over BOE for removing the oxide layer (both the plasma dry etching and the vapor HF etching were 
tried). However, it was found that the PECVD deposited nitride layer was being etched at a very high rate (higher than a thermally grown oxide layer), which made it difficult to go further with the dry etching process. On the other hand, the BOE solution etching rate for the deposited nitride layer was about $20 \mathrm{~nm} / \mathrm{min}$, which was five times slower than the measured etch rate for the oxide layer. Consequently, the wet buffered oxide etching process was selected to remove the oxide layer from the sample.

The sample was processed in the BOE solution for about 40 minutes to make sure that all oxide layer is etched away from the substrate. The wafer was then put in the water and dried in the oven for 15 and 30 minutes respectively. The last step was debonding the supporting wafer from the sample, which was bonded by a photoresist. To avoid any pressure on the released devices, which may result in breaking them, the sample was rested into an acetone bath for a couple of days to make sure that all the cured photoresist is dissolved and the supporting wafer could be easily detached from the sample without enforcing any pressure. The released sample was then dried in the oven for an hour as the final step of the fabrication process.

To summarize this section, the whole fabrication process performed in this study to build the designed MEMS bistable device is listed as follows:

1. Deposit the stressed nitride as the structural layer (PECVD).

2. Pattern the stressed nitride with a photomask (photolithography).

3. Etch the stressed nitride (dry etching).

4. Deposit the molybdenum as the bottom electrode layer (PVD sputtering). 
5. Pattern the molybdenum with a photomask (photolithography).

6. Etch the molybdenum (dry etching).

7. Deposit the aluminum nitride as the piezoelectric layer (PVD sputtering).

8. Pattern the aluminum nitride with a photomask (photolithography).

9. Etch the aluminum nitride (wet etching).

10. Deposit the molybdenum as the top electrode layer (PVD sputtering).

11. Pattern the molybdenum with a photomask (photolithography).

12. Etch the molybdenum (dry etching).

13. Pattern the backside of the wafer with a photomask (photolithography).

14. Etch the back oxide layer (BOE wet etching).

15. Bond a supporting wafer to the topside surface (photoresist bonding).

16. Etch through the whole silicon thickness from the backside (DRIE/Xactix $\mathrm{XeF}_{2}$ ).

17. Etch the top oxide layer (BOE wet etching).

18. Detach the supporting wafer and dry the released sample (acetone bath/oven).

\subsection{Results and Discussion}

The mask layouts included three different sizes of the bistable energy harvester as well as some clamped-clamped and cantilevered beams and several longer versions of the bistable device (with four cantilever arms and two or three torsional rods), a schematic picture of which is shown in the next chapter. Figure 62 shows the microscopic image of the topside 
fabricated device before the backside bulk etching process. It shows that all the topside layers of the device were successfully fabricated without any major problems. As can be noticed from the previous section and this figure, most of the fabrication issues are related to the final release of the device step due to several reasons including high compressive stress in the structural layer, difficulties of etching the top thermally grown oxide layer in the sample, detaching the supporting wafer, etc.

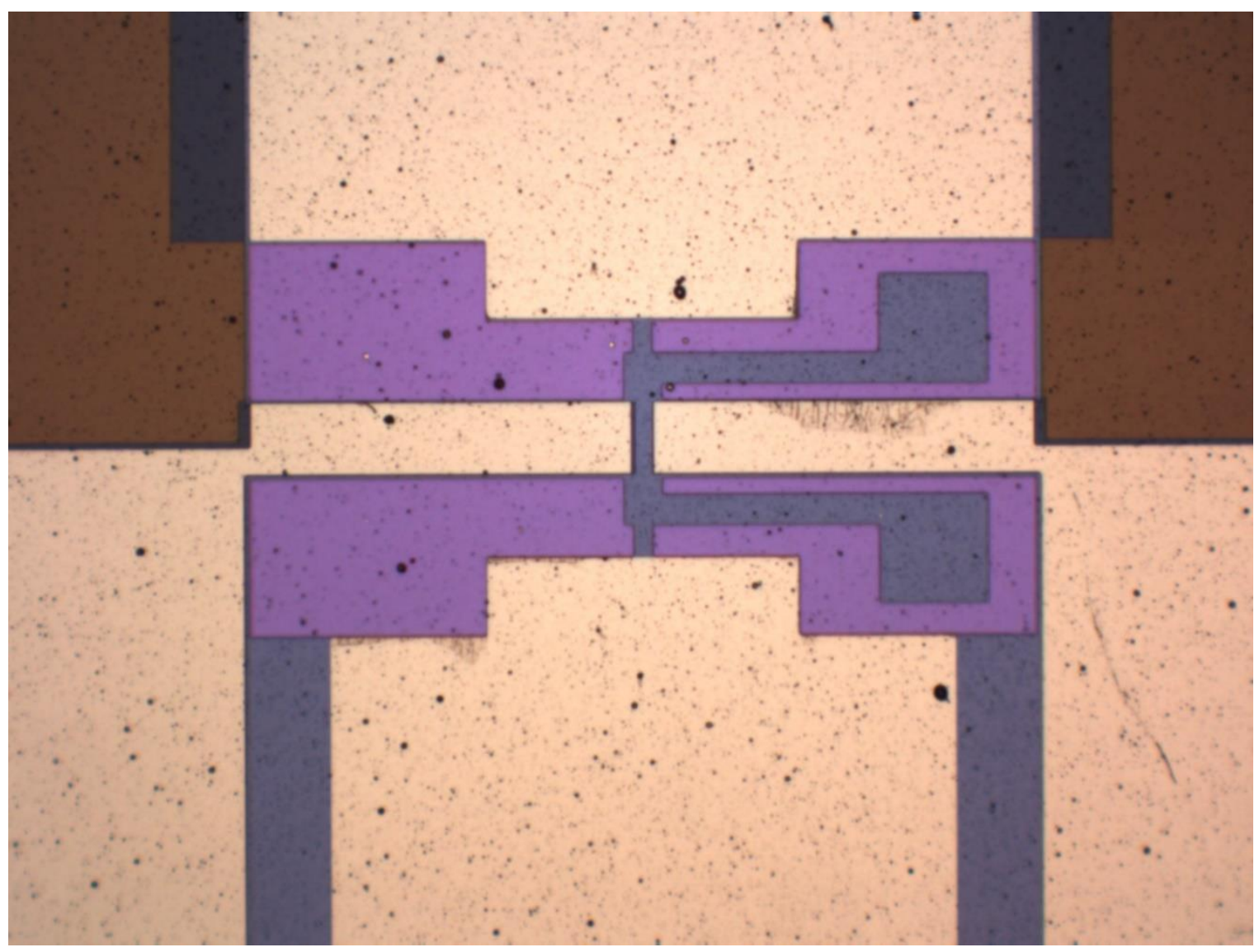

Figure 62. Microscopic topside view of the fabricated MEMS bistable energy harvester before doing the backside bulk etching process.

Figures 63 and 64 show the picture of the backside of the wafer and some microscopic images of bistable devices after the DRIE process respectively. By looking at Figure 63, it 
can be noticed that the DRIE process was performed successfully through the whole thickness of the silicon layer. Moreover, microscopic images (Figure 64) show the topside fabricated device from a back view, which confirms this statement and indicates that all the silicon layer was etched away from the sample after the DRIE process. However, some crack-shape lines are observed in these images. Since the supporting wafer was still bonded to the sample at this stage, it is hard to say that if these lines are the possible cracks in either the top oxide layer or any layer of the device, or they are related to the existing photoresist film between the sample and the supporting wafer.

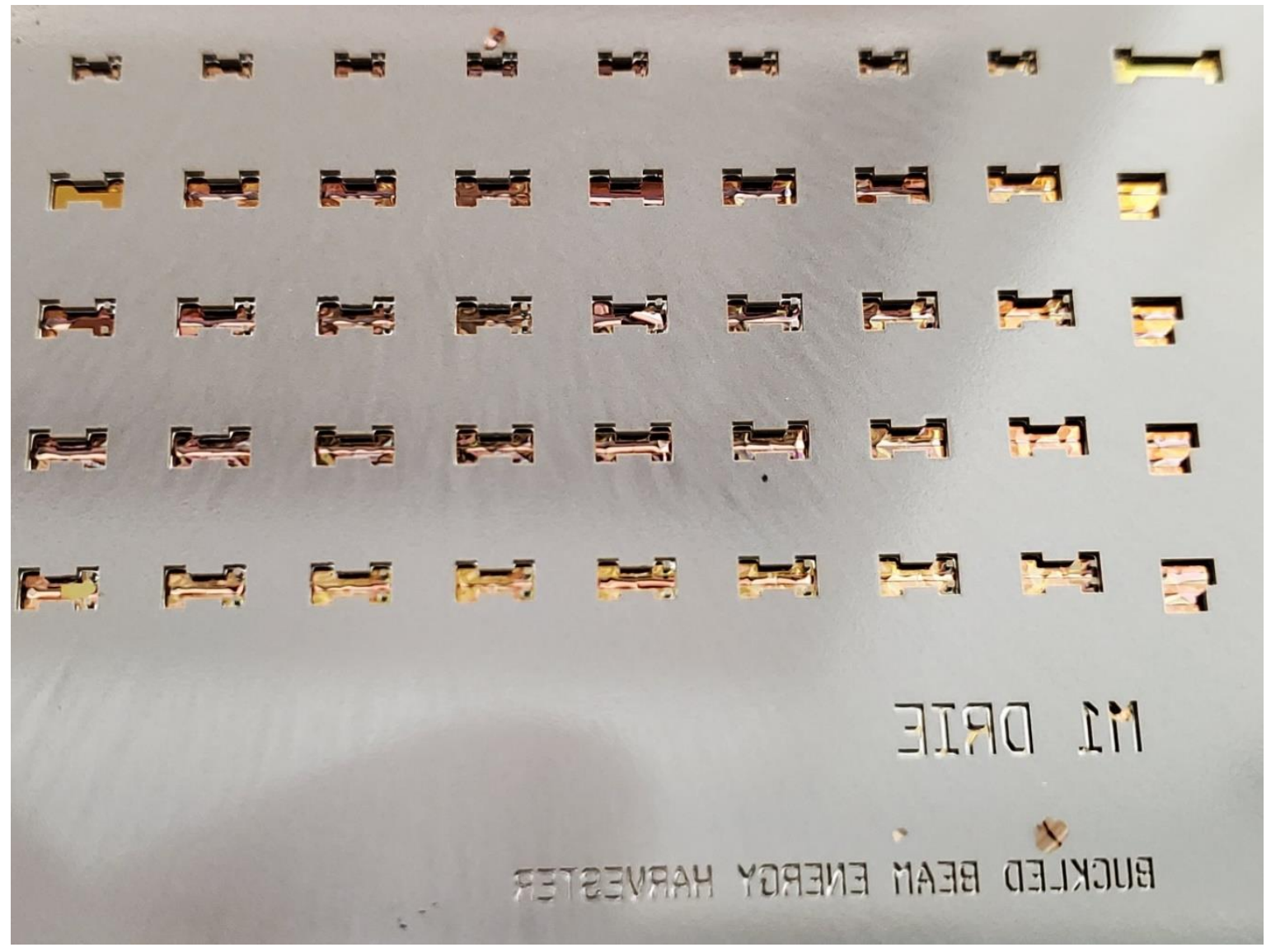

Figure 63. Backside view of the fabricated wafer after the DRIE process. 


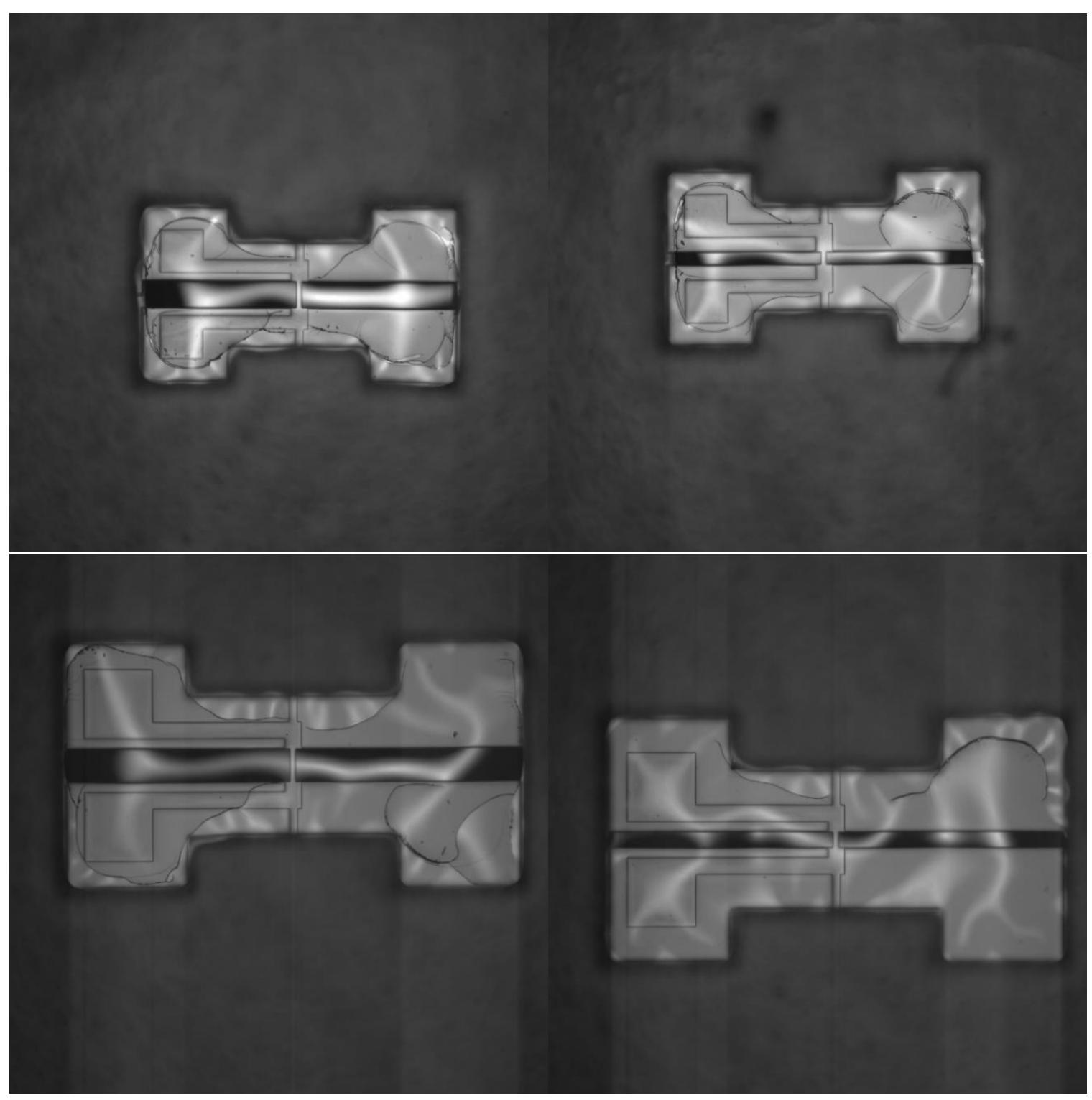

Figure 64. Backside microscopic images of some of the fabricated devices after the DRIE process.

A microscopic image of the final fabricated device after detaching the supporting wafer is shown in Figure 65. As can be observed from this figure, the bistable device was successfully released from its substrate. The reflected light coming from the main beam in these pictures confirms that the central beam is buckled in its second mode shape as predicted in the nonlinear static analysis of the MEMS device in chapter 5. Furthermore, it 
can be seen that some parts of the top electrode layers were being over etched in the topside fabrication process. This figure verifies the validity of the whole fabrication process discussed in this chapter to build the designed MEMS-scale vibration energy harvester.

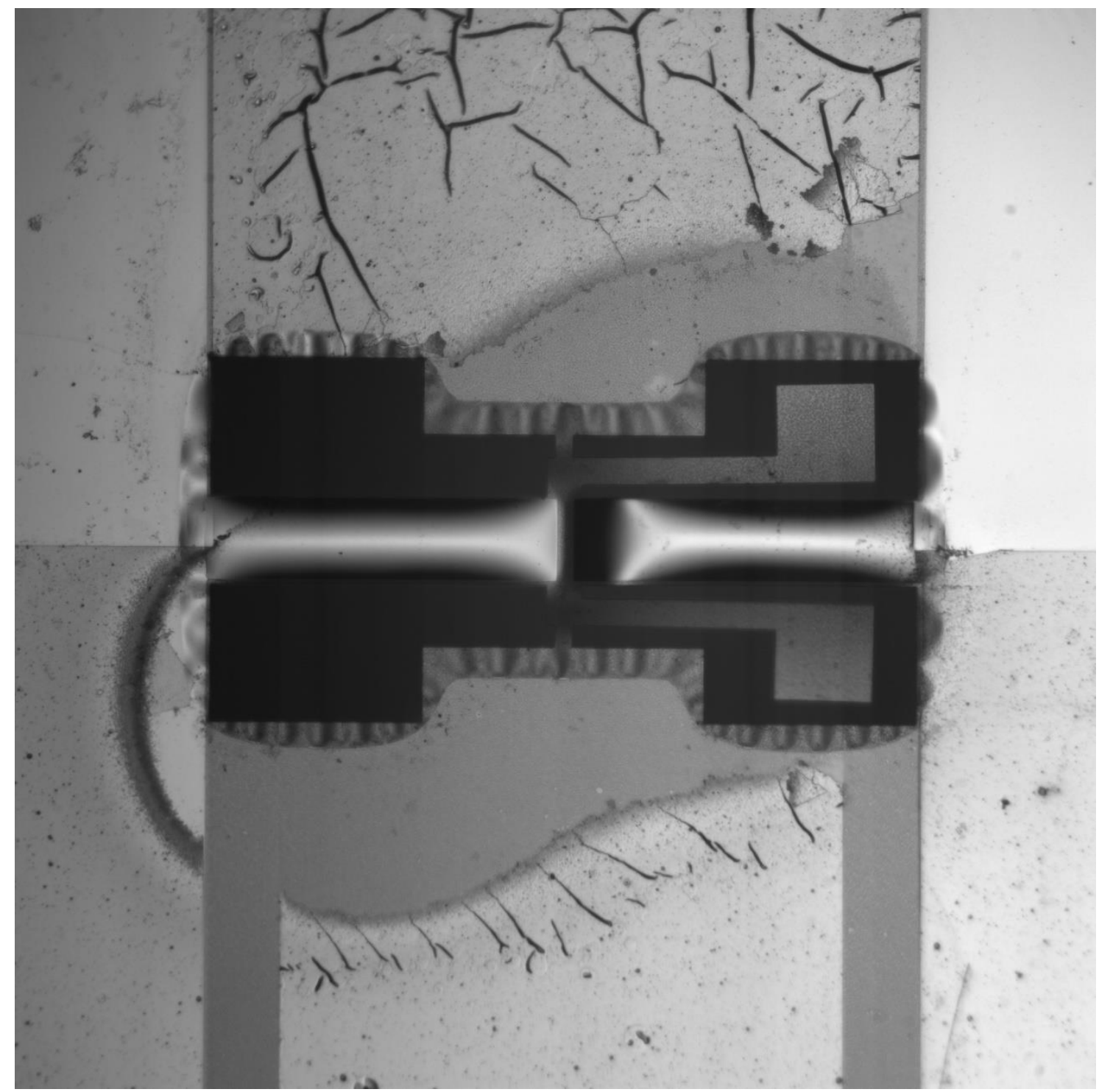

Figure 65. A topside microscopic image of the final fabricated device after the final release.

This chapter discusses the micro-fabrication steps required to make the studied MEMS bistable energy harvester. The fabrication processes, as well as the challenges faced during 
each process, were explained thoroughly in the previous section, and eventually, a summarized version of the whole fabrication process was listed based on the available facilities at the UofL Micro/Nano Technology Center. Several microscopic and large scale pictures of the final sample are shown and the experimental findings from these pictures are discussed in this section. One of the important experimental observations in this study is that out of the three different sizes in the fabricated bistable devices, more of the smaller ones survived after the final release. Since the thickness of the layers, as well as the compressive stress for all the fabricated devices, are assumed to be the same in general, it can be concluded that the smaller devices could survive better due to the lower compressive force applied to the central buckled beam. The suggested fabrication process in this study is based on our current facilities and the limited number of experimental tests performed for this project, which eventually led to the successful fabrication of the designed MEMS bistable energy harvester (Figure 65). However, there are several steps could be changed or optimized in the process including lowering the compressive stress, increasing the structural layer thickness, changing the order of the deposition, adding a more flexible material between the top oxide and the stressed silicon nitride layer, etc., which could help the fabrication process performed in a more robust and repeatable way and some of them will be discussed in the next chapter as the possible future works of this study. 


\section{SUMMARY, CONCLUSION, AND FUTURE WORKS}

\subsection{Summary}

The main purpose of this study is to propose, model, and fabrication of a uniquely designed bistable structure suitable for low-frequency MEMS vibration energy harvesting applications. In chapter 2, first, previously studied works was reviewed to highlight the current issues of the typical bistable energy harvesters under real-life conditions. Then, a coupled structure was introduced to tackle the mentioned problems associated with the bistable systems. This coupled structure consists of a central buckled beam with two stable buckled configurations. It has been shown that this design could solve some of the difficulties in generating snap-through motion at low frequencies presented by typical buckled structures used as vibration energy harvesters by enabling bistable motion in the system more easily via the motion of the coupled cantilever arms. The two cantilever arms with masses concentrated at their ends are connected to the central beam in a way makes the $1^{\text {st }}$ natural frequency easier to tailor to a particular vibration environment and the net result is that bistable motion is more easily facilitated over a range of low driving frequencies and amplitudes.

In order to better understand the dynamic behavior of the proposed structure, an accurate model is required to show the system response under various conditions, which consequently would lead to a more optimized device suitable for real-life applications. In 
the next chapter, a dynamic analysis of the structure was developed based on nonlinear Euler-Bernoulli beam theory. The equations of motion for each component was derived under harmonic excitation. A Galerkin's approach was chosen to discretize the obtained nonlinear partial differential equations, which brings up the requirement for a set of spatial functions. The functions considered for the presumed solutions satisfied only the essential boundary conditions of the system. To validate the proposed model, an experimental setup was also presented. While experimental results have shown the superior performance of such structure as a low-frequency bistable energy harvester, the proposed model failed to show the significant observations in the experiment. It was notified that this inconsistency happened as a result of not considering the true shape functions of the coupled system.

In chapter 4, a new component coupling method was introduced as an alternative to the previously proposed model. In this new approach, instead of using approximate shape functions for each component, it has been decided to find the exact theoretical shape functions of the linearized system being able to satisfy all the geometrical and force/moment boundary conditions at the connections. This requires solving the nonlinear static and linearized buckled dynamic of the proposed structure, which was accurately solved and validated by comparison with both finite element analysis (FEA) and experimental results. After validation of the model, snap-through regime plots were developed for the tested device under various conditions. The results showed the effect of different parameters, including buckling level of the central beam and exciting conditions, on the dynamic response of the bistable system.

Since the proposed structure was initially designed for MEMS energy harvesting applications, where lowering the operating frequency is one of the main challenges with 
bistable devices, chapter 5 discussed the analysis of a designed MEMS version of the device based on the developed coupling component approach. As an actual harvester is made of several layers including structural, piezoelectric, and electrode layers, the calculation of mechanical elongation of the piezoelectric layer and the estimated power output for an external pure resistive load was considered in this chapter. It was shown that having bistable motion in the system could result in a noticeable increase in mechanical elongation, harvested power, and consequently the performance of the micro-scale device.

To show the generality and validity of the proposed coupling method, it was applied to a flexible bistable beam made of PVDF, a polymer with piezoelectric properties, with a central mass as its main structural layer in chapter 6 . Since the main structure was made of piezoelectric material, coupled electro-mechanical governing equations based on the nonlinear Euler-Bernoulli beam theory were derived for the studied bistable system. An experimental setup was made from a commercial PVDF sheet and tested to find its various mechanical/electrical parameters. Vibrational amplitude and voltage response were experimentally measured under various loading conditions and the results were compared with the ones obtained from the modeling approach. A good agreement between the model and experiment as well as the existence of second resonances in the nonlinear system, which was successfully predicted by the component coupling method, verified the proposed approach and its generality for more complex coupled structures.

Chapter 7 discussed the microfabrication process taken to build the designed MEMS-scale bistable energy harvester. As shown in this chapter, the final device is made of four layers and required five mask layouts in total to be patterned and fabricated. The details of the fabrication process including deposition, photolithography, and etching of each layer, the 
final backside release, and the challenges faced in each step were thoroughly discussed and as a result, a list of the steps required for the whole microfabrication process of the studied energy harvester was proposed based on the facilities available at the University of Louisville Micro/Nano Technology Center. While certain areas can be imporved in the fabrication process, the microscopic images of the final fabricated device validate the steps taken in this study for making the micro-scale energy harvester.

\subsection{Conclusion and Future Works}

One of the main accomplishments of this study was developing a new component coupling approach with the ability to find the exact shape functions of complex coupled structures, particularly the designed bistable structure introduced in this project, which could result in a more accurate dynamic analysis of them. Another main achievement was fabricating a micro-scale version of the proposed bistable energy harvester to tackle some of the issues with the previously studied bistable structures at low-frequency ranges. Several challenges faced during the fabrication were reviewed and the final order of the process was briefed based on the facilities and experiments performed at the UofL Micro/Nano Technology Center.

As discussed earlier, the proposed coupling approach can be employed for the analysis of other complex structures. One of the systems that can be considered for future studies is an extended version of the presented bistable structure, a schematic view of which is shown in Figure 66. Since the central buckled beam has the essential role of harvesting energy due to their large strain variations, they could be stretched out in a series format to increase the harvesting potential of the device. As for the modeling part, since the structure is only expanded into more individual components, it will increase the number of generalized 
coordinates, the size of the coupling transformation matrix, and consequently the computational efforts for such a system. To have a better idea about its experimental fabrication process, some mask layouts were designed for the micro-scale version of the device. Figure 67 shows the microscopic pictures of the final fabricated device for this extended version. As can be seen from these pictures, the final released device was broken in some parts. This is due to the highly compressive stress in the long slender beams, resolving of which requires a more supportive structural layer with less induced stress.

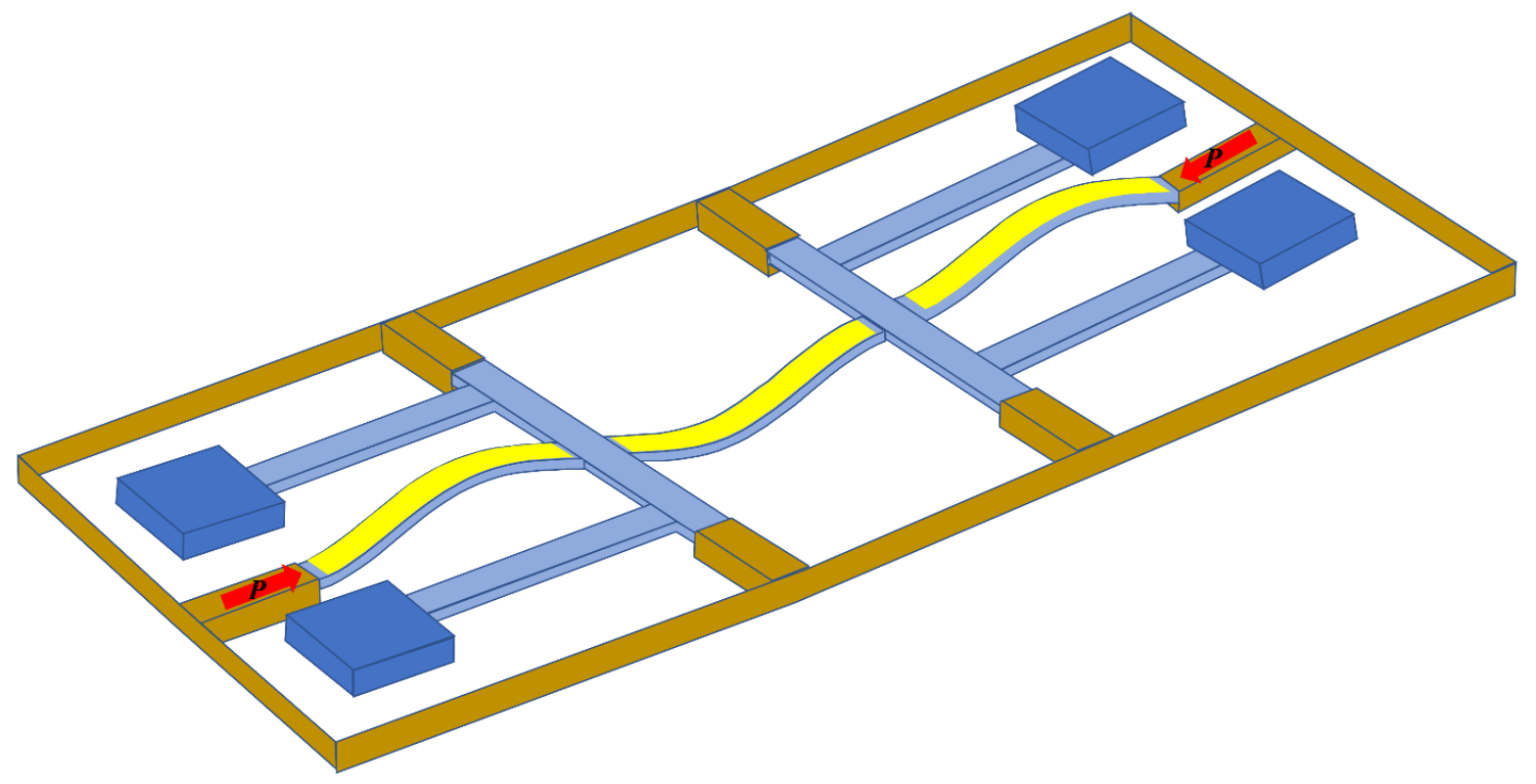

Figure 66. A schematic view of an extended version of the studied bistable energy harvester with a longer central beam and four cantilever arms. 


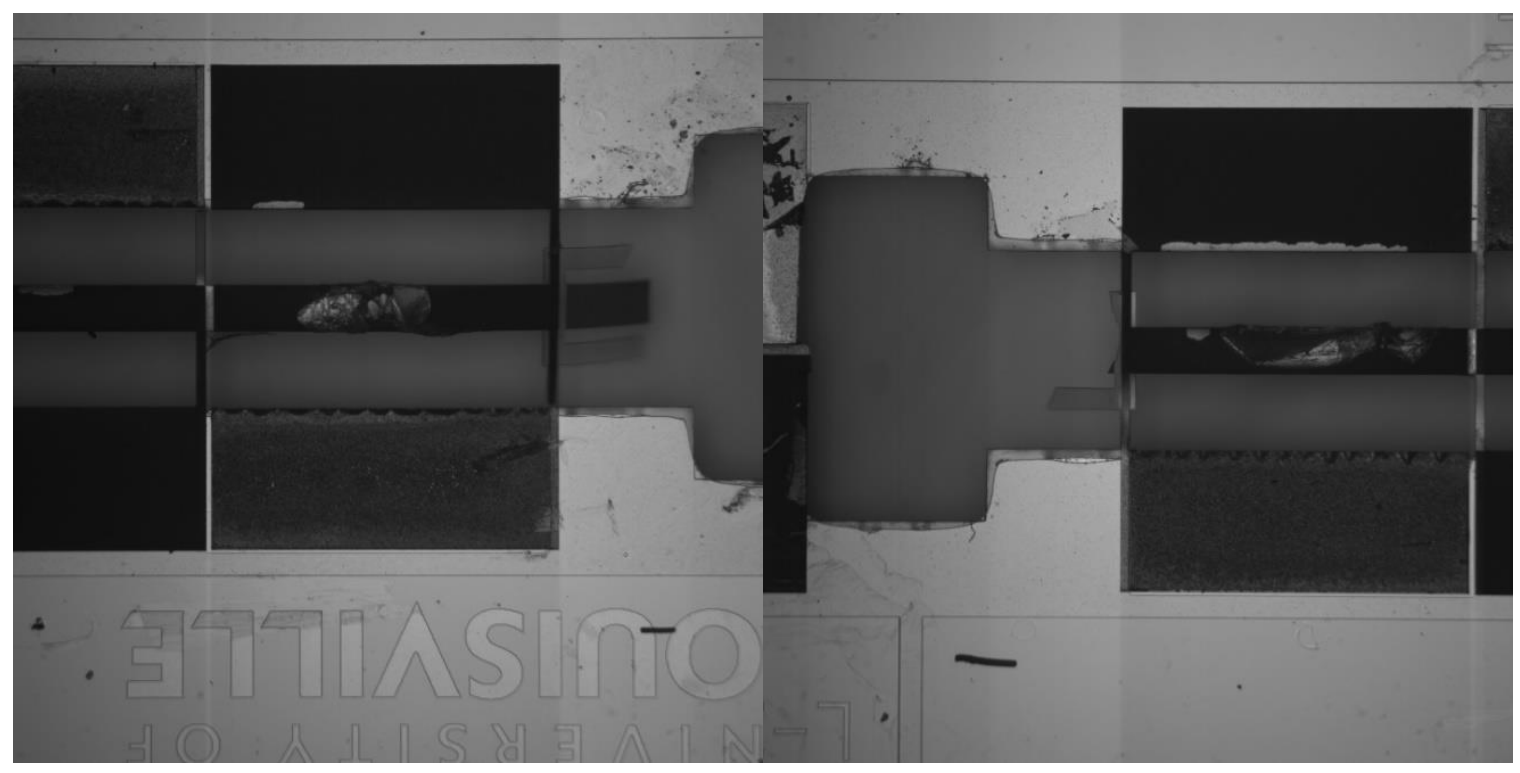

Figure 67. Microscopic pictures of the designed extended version of the bistable energy harvester after the final release.

Another thing that can be considered as the possible future work is the improvement of the microfabrication process to obtain a more robust and repeatable way for making the designed MEMS bistable energy harvester. While a successful MEMS device was fabricated by the experimental methods performed in this study, many issues occurred in the fabrication process which can be optimized and updated for future efforts. There were many broken devices observed after releasing them from their substrates (Figure 68). These broken devices were outnumbered for larger ones as a higher compressive force applied to their components. This issue occurred as a result of insufficient support from the structural layer, which can be resolved by increasing the thickness of the stressed nitride and/or adding some soft material such as polyimide (PI) to improve the rigidity/flexibility of the bistable structure. It must be paid attention that the latter option decreases the effective 
stress in the final fabricated device, and it requires a preliminary calculation of the total stress/thickness to make sure of the bistability occurrence after the final release.

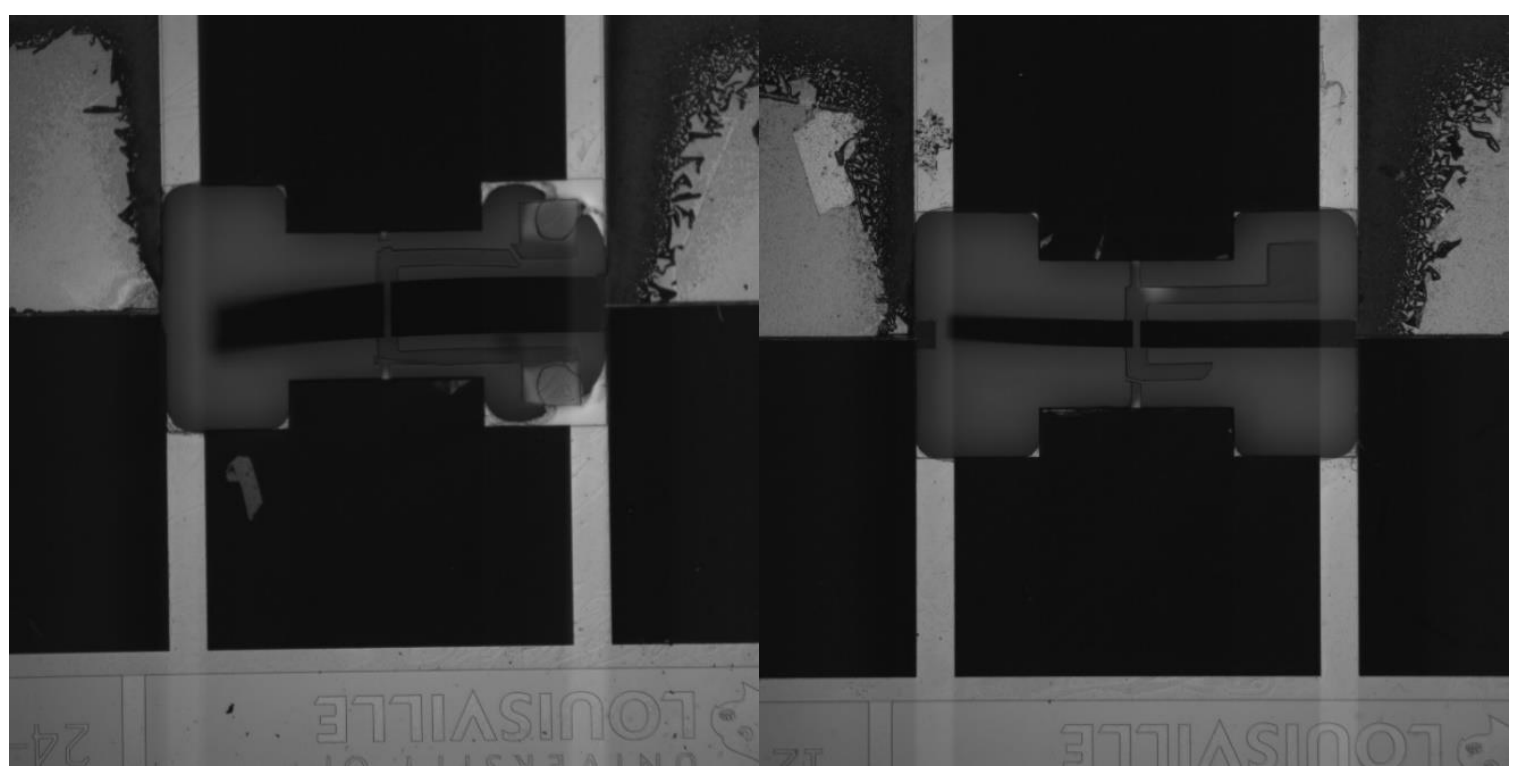

Figure 68. Microscopic pictures of some broken devices after the final release.

Since the main purpose of the MEMS device is harvesting energy from mechanical strain, the better the quality of the piezoelectric layer is, the higher the power output will be harvested. Among all the thin-film manufacturable piezoelectric materials, lead zirconate titanate (PZT) has the highest piezoelectric effects, which makes it one of the best options for energy harvesting purposes. However, as explained in the previous chapter, its deposition must be performed at a high temperature, which could end up losing the induced stress in the structural layer as a result of some unwanted annealing process. One way to solve this issue is by changing the order of the fabrication process and setting the compressive structural layer as the final deposited layer of the device. This requires some fundamental changes in its mask layout as the electrode pads must be exposed to the top surface of the device for the electrical connections. Furthermore, the performance of the 
fabricated micro-scale system needs to be experimentally tested where the results can be compared with the ones obtained from the modeling in chapter 5. This experimental validation requires a more advanced shaker table with a much wider frequency domain and higher resolution, which can also be considered as a continuing effort of this research for the future. 


\section{REFERENCES}

1. $\quad$ Erturk, A. and D.J. Inman, Piezoelectric energy harvesting. 2011: John Wiley \& Sons.

2. Kim, H.S., J.-H. Kim, and J. Kim, A review of piezoelectric energy harvesting based on vibration. International journal of precision engineering and manufacturing, 2011. 12(6): p. 1129-1141.

3. Wang, R.L.H.a.K., A review of the recent research on vibration energy harvesting via bistable systems. Smart Mater. Struct., 2013. 22: p. 023001.

4. Challa, V.R., et al., A vibration energy harvesting device with bidirectional resonance frequency tunability. Smart Materials and Structures, 2008. 17(1): p. 015035.

5. Abdelkefi, A., et al., An energy harvester using piezoelectric cantilever beams undergoing coupled bending-torsion vibrations. Smart Materials and Structures, 2011. 20(11).

6. Roundy, S. and P.K. Wright, A piezoelectric vibration based generator for wireless electronics. Smart Materials and structures, 2004. 13(5): p. 1131.

7. Shu, Y. and I. Lien, Analysis of power output for piezoelectric energy harvesting systems. Smart materials and structures, 2006. 15(6): p. 1499.

8. Pellegrini, S.P., et al., Bistable vibration energy harvesters: a review. Journal of Intelligent Material Systems and Structures, 2013. 24(11): p. 1303-1312.

9. Cao, D., S. Leadenham, and A. Erturk, Internal resonance for nonlinear vibration energy harvesting. The European Physical Journal Special Topics, 2015. 224(1415): p. 2867-2880.

10. Gammaitoni, L., I. Neri, and H. Vocca, Nonlinear oscillators for vibration energy harvesting. Applied Physics Letters, 2009. 94(16): p. 164102.

11. Harris, P., et al., Dynamics of a vibrational energy harvester with a bistable beam: voltage response identification by multiscale entropy and " $0-1$ " test. The European Physical Journal Plus, 2016. 131(4): p. 109.

12. Cottone, F., et al., Bistable electromagnetic generator based on buckled beams for vibration energy harvesting. Journal of Intelligent Material Systems and Structures, 2014. 25(12): p. 1484-1495. 
13. Andò, B., et al., Experimental and theoretical investigation of a nonlinear vibrational energy harvester. Procedia engineering, 2015. 120: p. 1024-1027.

14. Van Blarigan, L. and J. Moehlis, Dynamic analysis of a buckled asymmetric piezoelectric beam for energy harvesting. Chaos: An Interdisciplinary Journal of Nonlinear Science, 2016. 26(3): p. 033107.

15. Kathpalia, B., et al., An experimentally validated model for geometrically nonlinear plucking-based frequency up-conversion in energy harvesting. Smart Materials and Structures, 2017. 27(1): p. 015024.

16. Derakhshani, M., B.E. Allgeier, and T.A. Berfield, Study on the Fabrication Process of a MEMS Bistable Energy Harvester Based on Coupled Component Structures, in Mechanics of Biological Systems \& Micro-and Nanomechanics, Volume 4. 2019, Springer. p. 75-79.

17. Emam, S.A. and A.H. Nayfeh, Nonlinear responses of buckled beams to subharmonic-resonance excitations. Nonlinear Dynamics, 2004. 35(2): p. 105-122.

18. Ghayesh, M.H., M. Amabili, and H. Farokhi, Global dynamics of an axially moving buckled beam. Journal of Vibration and Control, 2015. 21(1): p. 195-208.

19. NAYFEH, S.A.E.a.A.H., On the Nonlinear Dynamics of a Buckled Beam Subjected to a Primary-Resonance Excitation. Nonlinear Dyn, 2004. 35: p. 1-17.

20. Nayfeh, A.H. and S.A. Emam, Exact solution and stability of postbuckling configurations of beams. Nonlinear Dynamics, 2008. 54(4): p. 395-408.

21. Woafo, B.R.N.N.a.P., Modeling and Optimal Active Control with Delay of the Dynamics of a Strongly Nonlinear Beam. Journal of Advanced Research in Dynamical and Control Systems, 2009. 1(1): p. 57-74.

22. Vangbo, M., An analytical analysis of a compressed bistable buckled beam. Sensors and Actuators A, 1998. 69: p. 212-216.

23. Azrar, L., R. Benamar, and R. White, Semi-analytical approach to the non-linear dynamic response problem of $S-S$ and $C-C$ beams at large vibration amplitudes Part I: General theory and application to the single mode approach to free and forced vibration analysis. Journal of Sound and Vibration, 1999. 224(2): p. 183207.

24. Yamaki, N. and A. Mori, Non-linear vibrations of a clamped beam with initial deflection and initial axial displacement, part I: theory. Journal of Sound and Vibration, 1980. 71(3): p. 333-346.

25. Feng, Y. and C. Bert, Application of the quadrature method to flexural vibration analysis of a geometrically nonlinear beam. Nonlinear Dynamics, 1992. 3(1): p. 13-18.

26. Ke, L.-L., J. Yang, and S. Kitipornchai, An analytical study on the nonlinear vibration of functionally graded beams. Meccanica, 2010. 45(6): p. 743-752. 
27. Hammad, B.K., Natural frequencies and mode shapes of mechanically coupled microbeam resonators with an application to micromechanical filters. Shock and Vibration, 2014. 2014.

28. Ilyas, S., et al., An experimental and theoretical investigation of electrostatically coupled cantilever microbeams. Sensors and Actuators A: Physical, 2016. 247: p. 368-378.

29. Hosseini-Hashemi, S., M. Derakhshani, and M. Fadaee, An accurate mathematical study on the free vibration of stepped thickness circular/annular Mindlin functionally graded plates. Applied Mathematical Modelling, 2013. 37(6): p. 41474164.

30. Garg, A. and S. Dwivedy, Nonlinear Dynamics of Axially Loaded Piezoelectric Energy Harvester. Procedia Engineering, 2016. 144: p. 592-599.

31. Liu, W., et al., Investigation of a buckled beam generator with elastic clamp boundary. Smart Materials and Structures, 2016. 25(11): p. 115045.

32. Erturk, A. and D. Inman, Broadband piezoelectric power generation on highenergy orbits of the bistable Duffing oscillator with electromechanical coupling. Journal of Sound and Vibration, 2011. 330(10): p. 2339-2353.

33. Samuel C. Stanton, C.C.M., Brian P. Mann, Nonlinear dynamics for broadband energy harvesting: Investigation of a bistable piezoelectric inertial generator. Physica D, 2010. 239: p. 640-653.

34. F Cottone, L.G., H Vocca, M Ferrari and V Ferrari, Piezoelectric buckled beams for random vibration energy harvesting. Smart Mater. Struct., 2012. 21: p. 035021.

35. H. Vocca, F.C., I. Neri, and L. Gammaitoni, A comparison between nonlinear cantilever and buckled beam for energy harvesting. Eur. Phys. J. Special Topics, 2013. 222: p. 1699-1705.

36. F. Cottone, M.M., H. Vocca, and L. Gammaitoni, Effect of boundary conditions on piezoelectric buckled beams for vibrational noise harvesting. Eur. Phys. J. Special Topics, 2015. 224(2855-2866).

37. Michael I Friswell, S.F.A., Onur Bilgen, Sondipon Adhikari, Arthur W Lees and Grzegorz Litak, Non-linear piezoelectric vibration energy harvesting from a vertical cantilever beam with tip mass. Journal of Intelligent Material Systems and Structures, 2012. 23(13): p. 1505-1521.

38. Qin, H.L.W., Dynamics and coherence resonance of a laminated piezoelectric beam for energy harvesting. Nonlinear Dyn, 2015. 81: p. 1751-1757.

39. Louis Van Blarigan, J.M.a.R.M., Low dimensional modeling of a non-uniform, buckled piezoelectric beam for vibrational energy harvesting. Smart Mater. Struct., 2015. 24: p. 065012. 
40. Barsallo, A.A.N., Nonlinear analysis and power improvement of broadband lowfrequency piezomagnetoelastic energy harvesters. Nonlinear Dyn, 2016. 83: p. 4156.

41. Ibrahim, S.W. and W.G. Ali, A review on frequency tuning methods for piezoelectric energy harvesting systems. Journal of renewable and sustainable energy, 2012. 4(6): p. 062703.

42. Bouhedma, S., et al., Magnetic Frequency Tuning of a Multimodal Vibration Energy Harvester. Sensors, 2019. 19(5): p. 1149.

43. Hara, M., et al. Power Estimation for Piezoelectric Energy Harvesters in Flexure Mode with Large Displacement Amplitude. in Journal of Physics: Conference Series. 2013. IOP Publishing.

44. Du, S., Y. Jia, and A. Seshia. Maximizing output power in a cantilevered piezoelectric vibration energy harvester by electrode design. in Journal of Physics: Conference Series. 2015. IOP Publishing.

45. Singh, K.A., R. Kumar, and R.J. Weber, A broadband bistable piezoelectric energy harvester with nonlinear high-power extraction. IEEE Transactions on Power Electronics, 2015. 30(12): p. 6763-6774.

46. Cai, X., et al., A critical analysis of the $\alpha, \beta$ and $\gamma$ phases in poly (vinylidene fluoride) using FTIR. RSC Advances, 2017. 7(25): p. 15382-15389.

47. Sencadas, V., R. Gregorio Jr, and S. Lanceros-Méndez, $\alpha$ to $\beta$ phase transformation and microestructural changes of PVDF films induced by uniaxial stretch. Journal of Macromolecular Science®, 2009. 48(3): p. 514-525.

48. Li, L., et al., Studies on the transformation process of PVDF from $\alpha$ to $\beta$ phase by stretching. RSC Advances, 2014. 4(8): p. 3938-3943.

49. Momenzadeh, N., H. Miyanaji, and T.A. Berfield, Influences of zirconium tungstate additives on characteristics of polyvinylidene fluoride (PVDF) components fabricated via material extrusion additive manufacturing process. The International Journal of Advanced Manufacturing Technology, 2019: p. 1-8.

50. Han, H., et al., Microstructure fabrication on a $\beta$-phase PVDF film by wet and dry etching technology. Journal of Micromechanics and Microengineering, 2012. 22(8): p. 085030.

51. Martins, P., A. Lopes, and S. Lanceros-Mendez, Electroactive phases of poly (vinylidene fluoride): Determination, processing and applications. Progress in polymer science, 2014. 39(4): p. 683-706.

52. Benz, M., W.B. Euler, and O.J. Gregory, The role of solution phase water on the deposition of thin films of poly (vinylidene fluoride). Macromolecules, 2002. 35(7): p. 2682-2688. 
53. Seminara, L., et al., Electromechanical characterization of piezoelectric PVDF polymer films for tactile sensors in robotics applications. Sensors and Actuators A: Physical, 2011. 169(1): p. 49-58.

54. Sappati, K. and S. Bhadra, Piezoelectric polymer and paper substrates: a review. Sensors, 2018. 18(11): p. 3605.

55. Mishra, S., et al., Advances in piezoelectric polymer composites for energy harvesting applications: a systematic review. Macromolecular Materials and Engineering, 2019. 304(1): p. 1800463.

56. Han, M., et al., Three-dimensional piezoelectric polymer microsystems for vibrational energy harvesting, robotic interfaces and biomedical implants. Nature Electronics, 2019. 2(1): p. 26.

57. Sultana, A., et al., Energy harvesting and self-powered microphone application on multifunctional inorganic-organic hybrid nanogenerator. Energy, 2019. 166: p. 963-971.

58. Klinge, U., et al., PVDF as a new polymer for the construction of surgical meshes. Biomaterials, 2002. 23(16): p. 3487-3493.

59. Shirinov, A. and W. Schomburg, Pressure sensor from a PVDF film. Sensors and Actuators A: Physical, 2008. 142(1): p. 48-55.

60. Miao, W., et al., Improved ultrafiltration performance and chlorine resistance of PVDF hollow fiber membranes via doping with sulfonated graphene oxide. Chemical Engineering Journal, 2017. 317: p. 901-912.

61. Numrich, U., et al., Pmma/pvdf film with particularly high weathering stability and high uv protective action. 2010, Google Patents.

62. Kang, G.-d. and Y.-m. Cao, Application and modification of poly (vinylidene fluoride)(PVDF) membranes-a review. Journal of membrane science, 2014. 463: p. $145-165$.

63. Betts, D., et al. Static and dynamic analysis of bistable piezoelectric-composite plates for energy harvesting. in 53rd AIAA/ASME/ASCE/AHS/ASC Structures, Structural Dynamics and Materials Conference 20th AIAA/ASME/AHS Adaptive Structures Conference 14th AIAA. 2012.

64. Gao, Y., et al., Performance of bistable piezoelectric cantilever vibration energy harvesters with an elastic support external magnet. Smart Materials and Structures, 2014. 23(9): p. 095003.

65. Leng, Y., et al., An elastic-support model for enhanced bistable piezoelectric energy harvesting from random vibrations. Journal of Applied Physics, 2015. 117(6): p. 064901.

66. Leadenham, S. and A. Erturk, Unified nonlinear electroelastic dynamics of a bimorph piezoelectric cantilever for energy harvesting, sensing, and actuation. Nonlinear Dynamics, 2015. 79(3): p. 1727-1743. 
67. Zorlu, Ö. and H. Külah, A MEMS-based energy harvester for generating energy from non-resonant environmental vibrations. Sensors and Actuators A: Physical, 2013. 202: p. 124-134.

68. $\mathrm{Xu}, \mathrm{R}$. and S. Kim. Low-frequency, low-G MEMS piezoelectric energy harvester. in Journal of Physics: Conference Series. 2015. IOP Publishing.

69. De Paula, A.S., D.J. Inman, and M.A. Savi, Energy harvesting in a nonlinear piezomagnetoelastic beam subjected to random excitation. Mechanical systems and signal processing, 2015. 54: p. 405-416.

70. Wang, Y., L. Peng, and Z. Huang, Structural optimum design of bistable cylindrical shell for broadband energy harvesting application. Theoretical and Applied Mechanics Letters, 2015. 5(4): p. 151-154.

71. Aridogan, U., I. Basdogan, and A. Erturk, Random vibration energy harvesting on thin plates using multiple piezopatches. Journal of Intelligent Material Systems and Structures, 2016. 27(20): p. 2744-2756.

72. Emad, A., et al., Testing and evaluation of stretching strain in clamped-clamped beams for energy harvesting. Smart Materials and Structures, 2016. 25(11): p. 115006.

73. Fang, H.-B., et al., Fabrication and performance of MEMS-based piezoelectric power generator for vibration energy harvesting. Microelectronics Journal, 2006. 37(11): p. 1280-1284.

74. Liu, J.-Q., et al., A MEMS-based piezoelectric power generator array for vibration energy harvesting. Microelectronics Journal, 2008. 39(5): p. 802-806.

75. Ferrari, M., et al., A single-magnet nonlinear piezoelectric converter for enhanced energy harvesting from random vibrations. Sensors and Actuators A: Physical, 2011. 172(1): p. 287-292.

76. Rezaeisaray, M., et al., Low frequency piezoelectric energy harvesting at multi vibration mode shapes. Sensors and Actuators A: Physical, 2015. 228: p. 104-111.

77. Zhou, S., et al., Analytical and experimental investigation of flexible longitudinal zigzag structures for enhanced multi-directional energy harvesting. Smart Materials and Structures, 2017. 26(3): p. 035008.

78. Porter, D.A., Bi-stable buckled energy harvesters actuated via torque arms. 2015.

79. Porter, D.A. and T.A. Berfield, A bi-stable buckled energy harvesting device actuated via torque arms. Smart Materials and Structures, 2014. 23(7): p. 075003.

80. Ginsberg, J.H., Mechanical and structural vibrations: theory and applications. 2001.

81. Apiwattanalunggarn, P., S.W. Shaw, and C. Pierre, Component mode synthesis using nonlinear normal modes. Nonlinear Dynamics, 2005. 41(1-3): p. 17-46. 
82. Porter, D.A. and T.A. Berfield, Constraint Effects on Torque-Actuated Bistable Energy Harvesters. Energy Harvesting and Systems, 2016. 3(1): p. 79-90.

83. Yarar, E., et al., Low temperature aluminum nitride thin films for sensory applications. AIP Advances, 2016. 6(7): p. 075115.

84. University of Louisville, Micro/Nano Technology Center. Website, . Available from: http://louisville.edu/micronano.

85. Polok-Rubiniec, M., K. Lukaszkowicz, and L. Dobrzański, Comparison of nanostructure and duplex PVD coatings deposited onto hot work tool steel substrate. Journal of Achievements in Materials and Manufacturing Engineering, 2010. 41(1-2).

86. Czekaj, D., et al., Deposition of PZT thin film and determination of their optical properties. Journal of the European Ceramic Society, 1999. 19(6-7): p. 1489-1492.

87. Shilpa, G., K. Sreelakshmi, and M. Ananthaprasad. PZT thin film deposition techniques, properties and its application in ultrasonic MEMS sensors: A review. in IOP Conference Series: Materials Science and Engineering. 2016. IOP Publishing.

88. Aardahl, C., et al., Electrical properties of AlN thin films deposited at low temperature on Si (100). Thin Solid Films, 1999. 346(1-2): p. 174-180.

89. Iriarte, G.F., J. Rodríguez, and F. Calle, Synthesis of c-axis oriented AlN thin films on different substrates: A review. Materials Research Bulletin, 2010. 45(9): p. 1039-1045.

90. Jackson, N., et al., Influence of aluminum nitride crystal orientation on MEMS energy harvesting device performance. Journal of Micromechanics and Microengineering, 2013. 23(7): p. 075014.

91. Cheng, H.-E., T.-C. Lin, and W.-C. Chen, Preparation of [0 0 2] oriented AlN thin films by mid frequency reactive sputtering technique. Thin Solid Films, 2003. 425(1-2): p. 85-89.

92. Ishihara, M., et al., Control of preferential orientation of AlN films prepared by the reactive sputtering method. Thin Solid Films, 1998. 316(1-2): p. 152-157.

93. Xu, X.-H., et al., Morphological properties of AlN piezoelectric thin films deposited by DC reactive magnetron sputtering. Thin Solid Films, 2001. 388(1-2): p. 62-67.

94. Marauska, S., et al., Sputtered thin film piezoelectric aluminium nitride as a functional MEMS material and CMOS compatible process integration. Procedia Engineering, 2011. 25: p. 1341-1344.

95. Khan, S., et al., Texture of the nano-crystalline AlN thin films and the growth conditions in DC magnetron sputtering. Progress in Natural Science: Materials International, 2015. 25(4): p. 282-290. 
96. Huang, C.-L., K.-W. Tay, and L. Wu, Aluminum nitride films deposited under various sputtering parameters on molybdenum electrodes. Solid-state electronics, 2005. 49(2): p. 219-225.

97. Akiyama, M., et al., Preparation of highly oriented aluminum nitride thin films on molybdenum bottom electrodes using metal interlayers. Journal of materials science, 2005. 40(5): p. 1159-1162.

98. Stoeckel, C., et al., Pulsed DC magnetron sputtered piezoelectric thin film aluminum nitride-Technology and piezoelectric properties. Journal of Applied Physics, 2014. 116(3): p. 034102.

99. Elfrink, R., et al., Vibration energy harvesting with aluminum nitride-based piezoelectric devices. Journal of Micromechanics and Microengineering, 2009. 19(9): p. 094005.

100. Zhao, X., et al., A vibration energy harvester using AlN piezoelectric cantilever array. Microelectronic Engineering, 2015. 142: p. 47-51.

101. Kim, H., et al., Fabrication of full-color GaN-based light-emitting diodes on nearly lattice-matched flexible metal foils. Scientific reports, 2017. 7(1): p. 1-5.

102. Xiong, F., et al., Transfer-Free Graphene-Like Thin Films on GaN LED Epiwafers Grown by PECVD Using an Ultrathin Pt Catalyst for Transparent Electrode Applications. Materials, 2019. 12(21): p. 3533.

103. Rais-Zadeh, M., et al., Gallium nitride as an electromechanical material. Journal of Microelectromechanical Systems, 2014. 23(6): p. 1252-1271.

104. Kang, J.-H., et al., Efficient energy harvesting of a GaN p-n junction piezoelectric generator through suppressed internal field screening. Journal of Materials Chemistry C, 2016. 4(15): p. 3337-3341.

105. Allgeier, B.E., Piezoelectric bistable buckled beam energy harvester. 2017.

106. Conn Center for Reneable Energy Research. Available from: https://www.conncenter.org/.

107. Abdallah, B., et al., Deposition of AlN films by reactive sputtering: Effect of radiofrequency substrate bias. Thin Solid Films, 2007. 515(18): p. 7105-7108.

108. Monteagudo-Lerma, L., et al., Two-step method for the deposition of AlN by radio frequency sputtering. Thin Solid Films, 2013. 545: p. 149-153.

109. Hasheminiasari, M. and J. Lin, Seed layer effect on the $\left(\begin{array}{lll}0 & 0 & 2\end{array}\right)$ texture and piezoelectric properties of pulsed-DC sputtered AlN thin films. Advances in Materials and Processing Technologies, 2016. 2(1): p. 66-72.

110. Mileham, J., et al., Wet chemical etching of AlN. Applied physics letters, 1995. 67(8): p. 1119-1121. 


\title{
CURRICULUM VITAE
}

Masoud Derakhshani

CONTACT INFORMATION

1900 Bashford Manor Ln

Louisville, KY 40218

\author{
+1 (502)296-4022 \\ masoud.derakhshani@louisville.edu \\ m.derakhshany87@gmail.com
}

\section{EDUCATION}

University of Louisville, Louisville, KY

Ph.D., Mechanical Engineering,

Iran University of Science and Technology, Tehran, Iran

M.S., Mechanical Engineering,

2009-2011

Babol Noshirvani University of Technology, Babol, Iran

B.S., Mechanical Engineering,

2005-2009

\section{RESEARCH INTERESTS}

- Smart materials and structures

- Structural dynamics and vibration

- MEMS modeling and fabrication

- Computational mechanics

- Nonlinear dynamics of flexible structures/soft robotics

- Finite element analysis (FEA)

\section{RESEARCH EXPERIENCES}

\section{University of Louisville}

- Modeling and fabrication of a uniquely designed MEMS piezoelectric vibration energy harvester (AlN and GaN were fabricated and tested as the piezoelectric material)

- Analytical and experimental analysis of a PVDF-based bistable energy harvester.

- Modeling, characterization and testing of additively manufactured PVDF beams as a vibration energy harvester. 
- Analytical and experimental analysis of low-frequency bistable structures suitable for low-frequency energy harvesting applications.

- Modeling of a MEMS bistable diaphragm as a thermally actuated sensor.

- Modeling and fabrication of a MEMS-scale nonlinear arch beam resonator as a memory device.

- Analytical and experimental modal analysis of a tennis racket.

\section{Iran University of Science and Technology}

- Analytical and finite element modal analysis of a stepped circular/annular Mindlin functionally graded plate.

- Analytical and finite element modal analysis of a moderately thick sectorial functionally graded plate.

- Dynamic analysis of different types of micro-beams in Atomic Force Microscope.

\section{PUBLICATIONS}

- M. Derakhshani, T.A. Berfield, K.D. Murphy, "A component coupling approach to dynamic analysis of a buckled, bistable vibration energy harvester structure" Nonlinear Dyn, 96(2):1429-1446, 2019.

- M. Derakhshani, T.A. Berfield, "Snap-through and mechanical strain analysis of a MEMS bistable vibration energy harvester" Shock and Vibration, vol. 2019, Article ID 6743676, 10 pages, 2019.

- M. Derakhshani, T.A. Berfield, "Dynamic behavior and output charge analysis of a bistable clamped-ends energy harvester" Nonlinear Structures and Systems, vol. 1. Springer, Cham, 2020. 273-277.

- M. Derakhshani, B.E. Allgeier, T.A. Berfield, "Study on the fabrication process of a MEMS bistable energy harvester based on coupled component structures" Mechanics of Biological Systems \& Micro-and Nanomechanics, vol. 4. Springer, 2019, 75-79.

- M. Derakhshani, T.A. Berfield, K.D. Murphy, "Dynamic analysis of a bistable buckled structure for vibration energy harvester" Dynamic Behavior of Materials, vol. 1. Springer, Cham, 2018. 199-208.

- M. Derakhshani, N. Momenzadeh, T.A. Berfield, "Analytical and experimental study of a clamped-clamped, bistable buckled beam low-frequency PVDF vibration energy harvester", Journal of Sound and Vibration (under review).

- D. Ratnayake, M. Derakhshani, T.A. Berfield, K.M. Walsh, "Bistability study of buckled MEMS diaphragm", Journal of Micromechanics and Microengineering (under review).

- N. Momenzadeh, M. Derakhshani, T.A. Berfield, "Electromechanical enhancement of material extrusion additively manufactured polyvinylidene fluoride structures", (ready for submission).

- Sh. Hosseini-Hashemi, M. Derakhshani, M. Fadaee, "An accurate mathematical study on the free vibration of stepped thickness circular/annular Mindlin Functionally graded plates", Appl Math Model, 37(6):4147-4164, 2013. 
- M. Derakhshani, Sh. Hosseini-Hashemi, M. Fadaee, "An analytical closed-form solution for free vibration of stepped circular/annular Mindlin functionally graded plate", 1st International Conference on Acoustics and Vibration (ISAV-2011), Amirkabir University of Technology, Tehran, Iran, Dec 2011.

- M. Derakhshani, B. Ghorbani, H. Salarian, "Modeling of Atomic Force Microscope (AFM) as a flexible continuous system using Euler-Bernoulli theory", Conference on Mechatronics and Robotics, Islamic Azad University, Nour, Iran, May 2011 (in Farsi).

- M. Derakhshani, B. Ghorbani, M. Mohammadi, "Modeling of piezo-tube actuators in nano-robots using theory of plates and shells", Conference on Mechatronics and Robotics, Islamic Azad University, Nour, Iran, May 2011 (in Farsi).

\section{CONFERENCE PRESENTATIONS}

- M. Derakhshani, N. Momenzadeh, T.A. Berfield, "Dynamics and output voltage analysis of a PVDF-based broadband bistable energy harvester", ASME 2019 Conference on Smart Materials, Adaptive Structures and Intelligent Systems (SMASIS), Omni Louisville, KY, USA, Sep 2019 (presentation).

- M. Derakhshani, T.A. Berfield, "Modeling and fabrication of a MEMS piezoelectric energy harvester" 2nd Annual Energy Harvesting Society Meeting (AEHSM), Philadelphia, PA, USA, Sep 2018 (presentation).

- M. Derakhshani, T.A. Berfield, "Nonlinear dynamics of a coupled bistable structure for vibration energy harvester" 2017 IWPMA/12th EHW/ 1st AEHSM, The Westin Tysons Corner, Falls Church, VA, USA Sep 2017 (presentation).

\section{TEACHING/WORKING EXPERIENCES}

- Graduate Research/Teaching Assistant, Mechanical Engineering Department, University of Louisville. (Courses: Mechanics of Materials Lab (Instructor), Mechanics of Materials, System Dynamics, Mechanical Vibration, Machine Design, Advanced Dynamics, Fracture Mechanics)

2018-2020

- Lecturer, Department of Mechanical Engineering, University of Payame Nour, Sari, Iran. (Courses: Statics, Dynamics, Thermodynamics, Machine Dynamics, Fluid Mechanics, Mechanical Vibrations, Mechanics of Materials) 2012-2015

- Lecturer, Department of Mechanical Engineering, Islamic Azad University, Behshahr, Iran (Courses: Fluid Mechanics). Spring 2012

- Exam Developer, Payame-Noor Center of Test Archives (Bank Soal PayameNoor), Tehran, Iran. 2013-2015

- Mechanical Engineering Intern, Research and Development Center, Saipa-Diesel Company, Tehran, Iran.

Summer 2009

\section{HONORS \& AWARDS}

- University Fellowship Award, University of Louisville. 
- Graduate Scholarship Award, University of Kansas.

- National Science Foundation (NSF) I-Corps Award, project title: "Dynamics and experimental analysis of a micro-scale bistable vibration energy harvester". 2017

- Ranked 2nd, ASME student presentation competition, University of Louisville.

\section{SKILLS}

\section{Experimental Skills}

- Various micro-fabrication and characterization skills: masks designs, photolithography, thin film deposition, dry/wet etching, deep reactive ion etching, thin film stress measurement, profilometry etc.

- Mechanical testing: bulk and micro-scale tensile testing, torsion testing, etc.

- High-speed digital image correlation (DIC).

- Experimental modal analysis.

\section{Computer Skills}

- Programming:

- Engineering software:

- Mathematic software:

- Others:
MATLAB.

ANSYS, Solidworks, CoventorWare, L-Edit.

MATLAB/Simulink, Mathematica.

Microsoft Office (Word, PowerPoint, Excel), Latex.

\section{SELECT COURSES}

\section{Undergraduate Courses}

- Mechanical vibrations (A+)

- Automatic control (A+)

- Advanced mechanics of materials (A+)

\section{Graduate Courses}

- MEMS design and fabrication $(\mathrm{A}+)$

- Finite element analysis (A+)

- Noise and vibration control (A+)

- Nonlinear vibrations $(\mathrm{A}+)$

- Advanced dynamics (A+) 